\title{
Theory of hard photoproduction
}

\author{
Michael Klasen* \\ II. Institut für Theoretische Physik, Universität Hamburg, D-22761 Hamburg, Germany
}

(Published 25 November 2002)

\begin{abstract}
The author reviews the present theoretical understanding of photons and hard photoproduction processes, discussing the production of jets, light and heavy hadrons, quarkonia, and prompt photons in photon-photon and photon-hadron collisions. Virtual and polarized photons and prompt-photon production in hadron collisions are also discussed. The most important leading-order and next-to-leading-order quantum chromodynamics results are compiled in analytic form. A large variety of numerical predictions is compared to data from TRISTAN, LEP, and HERA and extended to future electron and muon colliders. The sources of all relevant results are collected in an extensive bibliography.
\end{abstract}

\section{CONTENTS}

I. Introduction

II. Photon Spectra

A. Bremsstrahlung

B. Beamstrahlung

C. Laser backscattering

III. Photon Structure
A. Evolution equations
B. Boundary conditions and factorization schemes
C. Hadronic solutions

IV. Jet Production
A. Cone and cluster algorithms
B. Single jets
C. Dijets
D. Three jets
E. Dijets with a leading neutron

V. Hadron Production
A. Fragmentation
B. Light hadrons
C. Heavy hadrons
D. Quarkonia

VI. Prompt-Photon Production
A. Fragmentation
B. Isolation
C. Inclusive photons
D. Photons and jets

VII. Related Topics
A. Virtual photons
B. Polarized photons
C. Prompt photons in hadron collisions

VIII. Summary

Acknowledgments

References
1221

1222

1222

1223

1224

1225

1225

1226

1227

1231

1231

1232

1240

1243

1245

1247

1247

1251

1251

1255

1259

1260

1262

1263

1264

1265

1265

1269

1272

1274

1274

1274

\section{INTRODUCTION}

The photon is a fascinating particle. It is the fundamental gauge boson of quantum electrodynamics and as such one of the best-studied elementary particles of the standard model of particle physics. However, in highenergy reactions the photon exhibits a complex hadronic

\footnotetext{
*Electronic address: michael.klasen@desy.de
}

structure, which is far less well understood. Investigations began more than 40 years ago with fixed-target experiments and soft vector-meson production, which established the vector-meson-dominance model of fluctuations between photons and vector mesons. With the advent of quantum chromodynamics (QCD) in the 1970s as the theory of strong interactions, the pointlike nature of the photon, its coupling to quarks, and the perturbative evolution of the photon structure moved to center stage. For some time it seemed possible to actually calculate the hadronic structure of the photon. Unfortunately it turned out that this was possible only at asymptotically large energies, which could not be reached with the available accelerators. In the mid 1980s, the photon-beam energies were high enough to prove that there were two different types of photon interactions. The photon could interact directly with the quarks and gluons in the hadronic target, but it could also resolve into a hadronic structure, and the partonic constituents of the photon could participate in the hard scattering. At about the same time, the two processes were found to be theoretically related. The pointlike photon structure was shown to be singular and in need of regularization by a nonperturbative hadronic boundary condition, which had to be determined from theoretical models or deep-inelastic electron-photon experiments at the $e^{+} e^{-}$colliders PETRA and PEP.

During the last 15 years, tremendous experimental and theoretical effort has been directed toward refining the picture of the photon, testing its structure, and using the photon as a tool for studying the production and properties of jets, light and heavy hadrons, quarkonia, and prompt photons. Experimentally this was facilitated by the construction and operation of the $e^{+} e^{-}$colliders TRISTAN and LEP, with center-of-mass energies between 58 and $210 \mathrm{GeV}$, and the HERA ep collider, with a center-of-mass energy of $300 \mathrm{GeV}$, which has just been upgraded for high-luminosity operation. The experimental results on the structure and interactions of the photon have been discussed at many general meetings and the topical PHOTON conferences, and they have recently been reviewed by several authors (Erdmann, 1997; Abramowicz and Caldwell, 1999; Nisius, 2000; 
Krawczyk, Staszel, and Zembrzuski, 2001). The experimental advances were paralleled by similar theoretical improvements. Until the mid 1980s, determinations of photon structure and calculations of photoproduction processes were performed only in leading order of perturbative QCD, and a review of the leading-order formalism exists for prompt-photon, jet, and particle production in hadron collisions (Owens, 1987). Since then, several photon parametrizations and many photoproduction calculations have been performed in next-toleading order (NLO). In comparisons with experimental data, these calculations have provided stringent tests on QCD as well as precise information about photon structure and fragmentation and about the formation of jets and hadrons (for short reviews see Kramer, 1996, 1998, 1999; Klasen, 1997a, 1999b; Kniehl, 1997).

While leading-order predictions are straightforward to calculate, they are unfortunately not very precise and can only be used to estimate hard photoproduction cross sections within factors of 2 . The reason for this is that leading-order QCD cross sections depend strongly on the renormalization scale in the strong coupling and on the factorization scales in the photon and proton parton densities, which are commonly varied by factors of 2 around the physical scale to estimate the relative theoretical error. Specific problems exist for jet and heavyflavor production. In leading order every jet corresponds to just one parton, and jet algorithms cannot be implemented. Only beyond leading order does this become possible by combining two or more partons into a single jet. Heavy flavors can be treated as massive or massless particles, and only in next-to-leading order can the logarithmic mass and scale dependences in fixed and variable flavor number schemes be made explicit. The large luminosities and trigger rates at modern high-energy colliders and detectors have led to great improvements in the statistical accuracy of the experimental data, which exceeds the theoretical precision of leading-order calculations. In next-to-leading order the uncertainties can be reduced to a reasonable level (a few percent). This precision is generally limited to scattering processes with four external legs, and it has now been reached for almost all photoproduction processes. Exceptions are the real photoproduction of three jets, two hadrons or photons, and various quarkonium states, and the virtual or polarized photoproduction of hadrons and prompt photons. In some cases NLO precision may be insufficient to describe the experimental data, as will be discussed later. It would be necessary to work at next-to-next-to-leading order (NNLO), but this precision has so far been feasible only for inclusive processes like the total rates of $e^{+} e^{-}$and $e p$ scattering into hadrons.

It is the aim of this review to give a complete theoretical description of hard photoproduction processes, ranging from the generation of real, slightly virtual, and polarized photons with lepton beams, through the current knowledge about photon structure, to the methods and applications of NLO QCD calculations for jet, hadron, and prompt-photon photoproduction. While the theoretical tools cannot be described in full detail, the main lines of argument and techniques are explained conceptually, and the most important results are stated explicitly. The predictions are compared only to the most recent and precise data from TRISTAN, LEP, and HERA in order to reach up-to-date conclusions about the applicability of QCD and the properties of jets, hadrons, and photons. Selected predictions are made for future accelerators like linear $e^{+} e^{-}$and circular muon colliders or a future $e p$ machine in order to provide a look ahead, and an extensive bibliography guides the reader to all of the relevant theoretical and experimental literature. In this sense, this review is intended to serve as a compendium of the current state of the art in hard photoproduction.

\section{PHOTON SPECTRA}

In the first generation of photoproduction experiments, real photons with energies below $60 \mathrm{GeV}$ were generated by pion decays or electron bremsstrahlung and scattered off nuclear targets in order to study soft particle production and total cross sections (Paul, 1992). Measurements of hard photoproduction of photons and mesons began with the CERN experiment NA14 at beam energies between 50 and $150 \mathrm{GeV}$ (Auge et al., 1986). Energies up to $400 \mathrm{GeV}$ were later reached at Fermilab. They led to a first observation of jets in photoproduction (Adams et al., 1994), but were still too small for definite tests of QCD. Center-of-mass energies of 200-300 GeV have recently been reached at the $e^{+} e^{-}$ colliders PETRA, PEP, TRISTAN, and LEP and at the ep collider HERA (see Table I). Here spacelike, almost real bremsstrahlung photons are exchanged during the hard collision. At future linear $e^{+} e^{-}$colliders large particle bunch densities are needed to reach high luminosities. Additional beamstrahlung photons will then be created before the hard interaction by the coherent action of the electromagnetic field of one bunch on the opposite one. If the electron beams are collided with additional high-energy laser beams, real photons can be produced through Compton scattering. Thus three different mechanisms can contribute to photon scattering at highenergy colliders: bremsstrahlung, beamstrahlung, and laser backscattering. In this section, the corresponding photon energy spectra will be discussed.

\section{A. Bremsstrahlung}

If the outgoing and incoming leptons in a hard scattering process are almost collinear, the calculation of the corresponding cross section can be considerably simplified by using the Weizsäcker-Williams or equivalentphoton approximation (for a review see Budnev et al., 1974). Current conservation and the small photon virtuality lead to a factorization of the lepton scattering cross section into a broadband photon spectrum in the lepton and the hard photon scattering cross section. As early as the 1920s, Fermi (1924) discovered the equivalence between the perturbation of distant atoms by the field of charged particles flying by and the perturbation due to 
TABLE I. Past and current high-energy $e^{+} e^{-}$and $e^{ \pm} p$ colliders and their experiments.

\begin{tabular}{lccccl}
\hline \hline Collider & Particles & $\sqrt{S} / \mathrm{GeV}$ & Operation & Laboratory & \multicolumn{1}{c}{ Experiments } \\
\hline SPEAR & $e^{+} e^{-}$ & $3-8$ & $1972-1990$ & SLAC & CRYSTAL BALL, MARK \\
DORIS & $e^{+} e^{-}$ & $3-11$ & $1973-1993$ & DESY & ARGUS, CRYSTAL BALL, PLUTO \\
PETRA & $e^{+} e^{-}$ & $12-47$ & $1978-1986$ & DESY & CELLO, JADE, PLUTO, TASSO \\
CESR & $e^{+} e^{-}$ & $8-12$ & $1979-?$ & Cornell & CLEO \\
PEP & $e^{+} e^{-}$ & $29-30$ & $1980-1990$ & SLAC & MARK, TPC/2 $\gamma$ \\
TRISTAN & $e^{+} e^{-}$ & $50-64$ & $1987-1995$ & KEK & AMY, TOPAZ, VENUS \\
SLC & $e^{+} e^{-}$ & $91-100$ & $1989-2000$ & SLAC & SLD \\
LEP & $e^{+} e^{-}$ & $91-209$ & $1989-2000$ & CERN & ALEPH, DELPHI, L3, OPAL \\
PEP-II & $e^{+} e^{-}$ & $8-14$ & $1999-?$ & SLAC & BABAR \\
KEKB & $e^{+} e^{-}$ & $8-11$ & $1999-?$ & KEK & BELLE \\
\hline HERA & $e^{ \pm} p$ & $296-332$ & $1992-?$ & DESY & H1, HERA-B, HERMES, ZEUS \\
\hline \hline
\end{tabular}

incident electromagnetic radiation. His semiclassical treatment was then extended to high-energy electrodynamics by Weizsäcker (1934) and Williams (1934) independently, who used a Fourier analysis to unravel the predominance of transverse over longitudinal photons radiated from a relativistic charged particle. In the 1950s, Curtis (1956) and Dalitz and Yennie (1957) gave the first field-theoretical derivations and applied the approximation to meson production in electron-nucleon collisions.

In the Weizsäcker-Williams approximation, the energy spectrum of the exchanged photons is given by

$$
\begin{aligned}
f_{\gamma / l}^{\text {brems }}(x)= & \frac{\alpha}{2 \pi}\left[\frac{1+(1-x)^{2}}{x} \ln \frac{Q_{\max }^{2}(1-x)}{m_{l}^{2} x^{2}}\right. \\
& \left.+2 m_{l}^{2} x\left(\frac{1}{Q_{\max }^{2}}-\frac{1-x}{m_{l}^{2} x^{2}}\right)\right] .
\end{aligned}
$$

The subleading nonlogarithmic terms (Kessler, 1975; Frixione et al., 1993) modify the cross section typically by $5 \% . \alpha=1 / 137$ is the electromagnetic fine-structure constant, $x=E_{\gamma} / E_{l}(l=e, \mu)$ is the energy fraction transferred from the lepton to the photon, $m_{l}$ is the lepton mass, and $Q_{\max }^{2}=E_{l}^{2}(1-x) \theta_{\max }^{2}$ is the maximal photon virtuality for lepton scattering angles below $\theta_{\max }$. This angle can be determined by tagging the outgoing lepton in the forward direction or by requiring that it be lost in the beam pipe (antitagging). When no information about the scattered lepton is available, one has to integrate over the whole phase space, thus allowing large transverse momenta and endangering the factorization property of the cross section. Bremsstrahlung photons can be generated by electron and muon beams, but for the latter the photon density is smaller by approximately a factor of 2 due to the larger muon mass (Klasen, 1997b).

\section{B. Beamstrahlung}

Future circular $e^{+} e^{-}$colliders with center-of-mass energies above $\sqrt{S}=500 \mathrm{GeV}$ would suffer from very high synchrotron radiation. They must therefore have a lin- ear design and dense particle bunches in order to still obtain large luminosities. Inside the opposite bunch, electrons and positrons experience transverse acceleration and radiate beamstrahlung. The spectrum is controlled by the beamstrahlung parameter

$$
Y=\frac{5 r_{e}^{2} E_{e} N}{6 \alpha \sigma_{z}\left(\sigma_{x}+\sigma_{y}\right) m_{e}},
$$

which is proportional to the effective electromagnetic field of the bunches and depends on the classical electron radius $r_{e}=\alpha / m_{e}=2.818 \times 10^{-15} \mathrm{~m}$, the beam energy $E_{e}$, the total number of particles in a bunch $N$, and the rms sizes of the Gaussian beam $\sigma_{x}, \sigma_{y}, \sigma_{z}$. For not too large $Y(Y \leqslant 5)$, Chen (1992) derived the approximate spectrum

$$
\begin{aligned}
f_{\gamma / e}^{\text {beam }}(x)= & \frac{1}{\Gamma\left(\frac{1}{3}\right)}\left(\frac{2}{3 \Upsilon}\right)^{1 / 3} x^{-2 / 3}(1-x)^{-1 / 3} e^{-2 x /[3 \Upsilon(1-x)]} \\
& \times\left\{\frac{1-\sqrt{\frac{\Upsilon}{24}}\left[1-\frac{1}{g(x) N_{\gamma}}\left(1-e^{\left.-g(x) N_{\gamma}\right)}\right]\right.}{}+\sqrt{\frac{\Upsilon}{24}}\left[1-\frac{1}{N_{\gamma}}\left(1-e^{\left.-N_{\gamma}\right)}\right]\right\}\right.
\end{aligned}
$$

with

$$
g(x)=1-\frac{1}{2}\left[(1+x) \sqrt{1+Y^{2 / 3}}+1-x\right](1-x)^{2 / 3} .
$$

The average number of photons radiated per electron throughout the collision is

$$
N_{\gamma}=\frac{5 \alpha^{2} \sigma_{z} m_{e} \Upsilon}{2 r_{e} E_{e} \sqrt{1+\Upsilon^{2 / 3}}} .
$$

Current design parameters for possible future $e^{+} e^{-}$colliders are listed in Table II, and the spectra corresponding to the $\sqrt{S}=500 \mathrm{GeV}$ designs are shown in Fig. 1 . This figure also displays the bremsstrahlung spectra for electrons and muons, which have been integrated over 
TABLE II. Current design parameters for possible future linear $e^{+} e^{-}$colliders (Adolphsen et al., 2000; Brinkmann et al., 2001; Kamitani and Rinolfi, 2001).

\begin{tabular}{cccccc}
\hline \hline Collider & TESLA & ILC-A & ILC-B & ILC-C & CLIC \\
Last update & $3 / 01$ & $4 / 00$ & $4 / 00$ & $4 / 00$ & $3 / 01$ \\
\hline$\sqrt{S}(\mathrm{GeV})$ & 500 & 535 & 515 & 500 & 500 \\
Particles/Bunch $\left(10^{10}\right)$ & 2 & 0.75 & 0.95 & 1.1 & 0.4 \\
$\sigma_{x}(\mathrm{~nm})$ & 553 & 277 & 330 & 365 & 202 \\
$\sigma_{y}(\mathrm{~nm})$ & 5 & 3.4 & 4.9 & 7.6 & 2.5 \\
$\sigma_{z}(\mu \mathrm{m})$ & 300 & 90 & 120 & 145 & 30 \\
$\Upsilon$ & 0.05 & 0.14 & 0.11 & 0.09 & 0.28 \\
\hline$\sqrt{S}(\mathrm{GeV})$ & 800 & 1046 & 1008 & 978 & 1000 \\
Particles/Bunch $\left(10^{10}\right)$ & 1.4 & 0.75 & 0.95 & 1.1 & 0.4 \\
$\sigma_{x}(\mathrm{~nm})$ & 391 & 197 & 235 & 260 & 115 \\
$\sigma_{y}(\mathrm{~nm})$ & 2.8 & 2.7 & 3.9 & 5.4 & 1.75 \\
$\sigma_{z}(\mu \mathrm{m})$ & 300 & 90 & 120 & 145 & 30 \\
$\Upsilon$ & 0.10 & 0.38 & 0.30 & 0.25 & 0.98 \\
\hline \hline
\end{tabular}

the photon virtuality up to an upper bound $Q_{\max }^{2}$ $=4 E_{l}^{2}(1-x)$ for untagged outgoing leptons. Both the bremsstrahlung and beamstrahlung spectra are soft, but for particular collider designs beamstrahlung can be more important than bremsstrahlung over a wide range in $x$. Beamstrahlung is completely negligible at muon colliders, since the parameter $\Upsilon_{\mu}=m_{e}^{3} / m_{\mu}^{3} \cdot \Upsilon_{e}$, which controls the spectrum mostly through the first exponential function in Eq. (2.3), is about five orders of magnitude smaller than for electrons (Klasen, 1997b).

\section{Laser backscattering}

Real photons of very high energy can be produced if laser photons are backscattered off electrons. The laser backscattering spectrum (Ginzburg et al., 1984),

$$
\begin{aligned}
f_{\gamma / e}^{\text {laser }}(x)= & \frac{1}{N_{c}+2 \lambda_{e} P_{c} N_{c}^{\prime}}\left[1-x+\frac{1}{1-x}-\frac{4 x}{X(1-x)}\right. \\
& \left.+\frac{4 x^{2}-2 \lambda_{e} P_{c} x(2-x) X[x(X+2)-X]}{X^{2}(1-x)^{2}}\right],
\end{aligned}
$$

where

$$
N_{c}=\left[1-\frac{4}{X}-\frac{8}{X^{2}}\right] \ln (1+X)+\frac{1}{2}+\frac{8}{X}-\frac{1}{2(1+X)^{2}}
$$

and

$$
N_{c}^{\prime}=\left[\left(1+\frac{2}{X}\right) \ln (1+X)-\frac{5}{2}+\frac{1}{1+X}-\frac{1}{2(1+X)^{2}}\right]
$$

are related to the total Compton cross section, depends on the center-of-mass energy of the electron-laser photon collision $s_{e \gamma}$ through the parameter $X=s_{e \gamma} / m_{e}^{2}-1$. The optimal value of $X$ is determined by the threshold for the process $\gamma \gamma \rightarrow e^{+} e^{-}$and is $X=2(1+\sqrt{2}) \simeq 4.83$ (Telnov, 1990). If this value is kept fixed, the laser backscattering spectrum becomes independent of $\sqrt{S}$. A large fraction of the photons is then produced close to the kinematic limit $x<x_{\max }=X /(1+X) \simeq 0.828$, so that one obtains an almost monochromatic "photon collider." The monochromaticity of the produced photons can be further improved if the helicities of the electron $\lambda_{e}$ and of the laser photon $P_{c}$ satisfy the condition

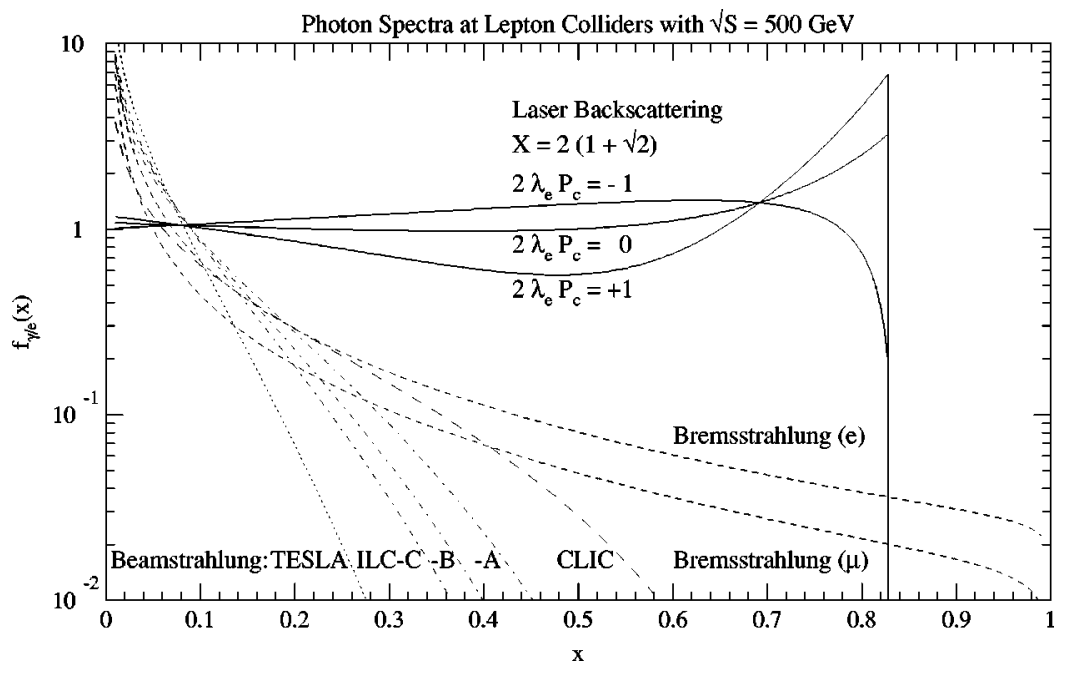

FIG. 1. Photon energy spectra at lepton colliders with $\sqrt{S}=500 \mathrm{GeV}$. The bremsstrahlung spectrum depends logarithmically on $\sqrt{S}$ and is roughly two times larger for electrons than for muons. The electron beamstrahlung spectra depend strongly on the design parameters and rise with $\sqrt{S}$. The laser backscattering spectrum is completely independent of $\sqrt{S}$ if the center-of-mass energy of the electronlaser photon collision is kept fixed, but it depends on the polarizations of the electron $\lambda_{e}$ and of the laser photon $P_{c}$. 
$2 \lambda_{e} P_{c}=-1$ (see Fig. 1). For electron beams, the optimal pulse energies $(\sim 1 \mathrm{~J})$ and repetition rates $(\sim 1 \mathrm{kHz})$ can be provided by current laser technology. For muon beams, one would need much higher flash energies $(\sim 1 \mathrm{GJ})$, which are currently not feasible (Klasen, 1997b). High-energy interactions of laser-backscattered photons have yet to be experimentally observed, and the simulation tools for realistic photon spectra including low-energy tails and nonlinear effects must be refined in order to estimate reliably the luminosities and event rates for photon colliders.

\section{PHOTON STRUCTURE}

The first generation of photoproduction experiments on nuclear targets revealed striking similarities with purely hadronic processes like pion scattering. The total cross sections showed strong resonances below centerof-mass energies of $3 \mathrm{GeV}$ and flat behavior above, and the elastic scattering cross sections fell strongly with the momentum transfer (Bauer et al., 1978). The most striking feature, however, was the copious production of vector mesons, particularly of the $\rho$ meson, which led Stodolsky (1964) to postulate an analogy between the isovector electromagnetic current and the $\rho$ field operator as well as a relation between diffractive $\gamma p$ and elastic $\rho p$ cross sections (Ross and Stodolsky, 1966). This analogy was subsequently extended in the vector-mesondominance (VMD) model, in which the photon was viewed as a superposition of $\rho, \omega$, and $\phi$ mesons with a small, if not negligible, pointlike contribution (Joos, 1967; Stodolsky, 1967). In the generalized vectordominance model (Sakurai and Schildknecht, 1972), all mesons, which carried the same quantum numbers as the photon $\left(J^{P C}=1^{--}\right)$, were included as contributions to the photon structure, even the heavier $J / \Psi$ meson.

The hadronic picture of the photon was revolutionized with the advent of QCD. Witten (1977) showed that the photon structure had an anomalous (pointlike) component, which could be understood as a short-time fluctuation into quark-antiquark pairs and gluons and could be calculated perturbatively. QCD also predicted direct photon scattering off quarks and gluons, which was subsequently observed in fixed-target experiments at higher energies and momentum transfers (Auge et al., 1986). Thus it became clear that the photon had a pointlike and a hadronic component, that they were important at different momentum transfers, and that they induced photon and parton scattering reactions, respectively (Brodsky et al., 1978, 1979; Lewellyn-Smith, 1978). Much of the present knowledge about the hadronic structure of the photon has been obtained from measurements of the photon structure functions $F_{2}^{\gamma}$ and $F_{L}^{\gamma}$ in the deepinelastic scattering process $e(k) \gamma(p) \rightarrow e\left(k^{\prime}\right) X$ at $e^{+} e^{-}$ colliders with

$$
\begin{aligned}
\frac{d^{2} \sigma}{d x d Q^{2}}= & \frac{2 \pi \alpha^{2}}{x Q^{4}}\left\{\left[1+(1-y)^{2}\right] F_{2}^{\gamma}\left(x, Q^{2}\right)\right. \\
& \left.-y^{2} F_{L}^{\gamma}\left(x, Q^{2}\right)\right\},
\end{aligned}
$$

where the flux of quasireal photons is given by the equivalent-photon approximation, Eq. (2.1), and where the kinematic variables $Q^{2}=-q^{2}=-\left(k-k^{\prime}\right)^{2}, \quad x$ $=Q^{2} /(2 p \cdot q)$, and $y=(p \cdot q) /(p \cdot k)$ are experimentally determined.

\section{A. Evolution equations}

In next-to-leading order of QCD, an initial-state photon can split into a massless quark-antiquark pair, which then interacts in the hard scattering, and a collinear singularity is encountered. In dimensional regularization (Bollini and Giambiagi, 1972a, 1972b; 't Hooft and Veltman, 1972; Gastmans and Meuldermans, 1973; Leibbrandt, 1975; Marciano, 1975; Marciano and Sirlin, 1975), where scattering matrix elements and phase-space factors are evaluated in $n=4-2 \varepsilon$ dimensions, the singularity manifests itself as a $1 / \varepsilon$ pole multiplying the spacelike photon-quark splitting function $P_{q \leftarrow \gamma}(x)$ in

$$
\begin{aligned}
\Gamma_{q \leftarrow \gamma}\left(x, M_{f}^{2}\right)= & \delta_{q \gamma} \delta(1-x) \\
& -\frac{1}{\varepsilon} \frac{\alpha}{2 \pi} \frac{\Gamma(1-\varepsilon)}{\Gamma(1-2 \varepsilon)}\left(\frac{4 \pi \mu^{2}}{M_{f}^{2}}\right)^{\varepsilon} \\
& \times P_{q \leftarrow \gamma}(x)+\mathcal{O}\left(\varepsilon, \alpha^{2}, \alpha \alpha_{s}\right) \\
= & \delta_{q \gamma} \delta(1-x)-\left[\frac{1}{\varepsilon}-\gamma_{E}+\ln (4 \pi)\right. \\
& \left.+\ln \frac{\mu^{2}}{M_{f}^{2}}\right] \frac{\alpha}{2 \pi} P_{q \leftarrow \gamma}(x)+\mathcal{O}\left(\varepsilon, \alpha^{2}, \alpha \alpha_{s}\right) .
\end{aligned}
$$

Here $x$ is the longitudinal momentum fraction of the quark in the photon, $\gamma_{E}=0.5772 \ldots$ is the Euler constant, and the scale $\mu$ has been introduced to preserve the dimension of physical quantities. The factorization theorem (Amati, Petronzio, and Veneziano, 1978a, 1978b; Ellis et al., 1979; Collins, Soper, and Sterman, 1988) ensures that the collinear singularity appearing in the transition function $\bar{\Gamma}$ is universal, i.e., independent of the leading-order scattering process, and can be absorbed into a renormalized quark density in the photon,

$$
f_{q / \gamma}\left(x, M_{f}^{2}\right)=\bar{f}_{q / \gamma}(x)+\left[\bar{\Gamma}_{q \leftarrow \gamma}\left(M_{f}^{2}\right) \otimes \bar{f}_{\gamma / \gamma}\right](x),
$$

at a factorization scale $M_{f}$. Thus logarithmic dependences on the artificial scale $M_{f}$ are induced in $f_{q / \gamma}$ and in the NLO partonic scattering cross section, which cancel up to higher orders in the perturbative expansion. In the modified minimal subtraction ( $\overline{\mathrm{MS}}$ ) scheme (Bardeen et al., 1978) no additional finite terms are subtracted, since these generally depend on the hard scattering process. In Eq. (3.3) the $x$-space convolution is defined as

$$
\left[\bar{\Gamma}_{j \leftarrow i} \otimes \bar{f}_{i / \gamma}\right](x)=\int_{x}^{1} \frac{d y}{y} \bar{\Gamma}_{j \leftarrow i}\left(\frac{x}{y}\right) \bar{f}_{i / \gamma}(y),
$$

$\bar{f}_{q / \gamma}(x)=\delta_{q \gamma} \delta(1-x)+\mathcal{O}(\alpha)$ is the bare-quark density, and $\bar{f}_{\gamma / \gamma}(x)=\delta(1-x)+\mathcal{O}\left(\alpha^{2}\right)$ is the bare-photon den- 
sity, which gets renormalized only at next-to-next-toleading order (NNLO) in $\alpha$. A renormalized gluon density in the photon $f_{g / \gamma}\left(x, M_{f}^{2}\right)$ is generated in a similar fashion by gluon radiation from a quark-antiquark pair at $\mathcal{O}\left(\alpha \alpha_{s}\right)$, where $\alpha_{s}\left(\mu^{2}\right)$ is the running coupling constant of quarks and gluons. Beyond leading order, the direct photon scattering processes are thus intimately connected to the resolved processes. In this section, we review the NLO QCD evolution equations, boundary conditions, factorization schemes, and hadronic solutions of the photon structure function.

The evolution of the parton densities in the photon with the scale $M_{f}^{2}$ can be calculated in perturbation theory by taking the logarithmic derivatives of $f_{q / \gamma}, f_{g / \gamma}$, and $f_{\gamma / \gamma}$ with respect to $M_{f}^{2}$. This leads to a coupled system of integro-differential equations (De Witt et al., 1979)

$$
\begin{aligned}
\frac{d f_{q / \gamma}\left(Q^{2}\right)}{d \ln Q^{2}}= & \frac{\alpha}{2 \pi} P_{q \leftarrow \gamma} \otimes f_{\gamma / \gamma}\left(Q^{2}\right)+\frac{\alpha_{s}\left(Q^{2}\right)}{2 \pi} \\
& \times\left[P_{q \leftarrow q} \otimes f_{q / \gamma}\left(Q^{2}\right)+P_{q \leftarrow g} \otimes f_{g / \gamma}\left(Q^{2}\right)\right], \\
\frac{d f_{g / \gamma}\left(Q^{2}\right)}{d \ln Q^{2}}= & \frac{\alpha}{2 \pi} P_{g \leftarrow \gamma} \otimes f_{\gamma / \gamma}\left(Q^{2}\right)+\frac{\alpha_{s}\left(Q^{2}\right)}{2 \pi} \\
& \times\left[P_{g \leftarrow q} \otimes f_{q / \gamma}\left(Q^{2}\right)+P_{g \leftarrow g} \otimes f_{g / \gamma}\left(Q^{2}\right)\right], \\
\frac{d f_{\gamma / \gamma}\left(Q^{2}\right)}{d \ln Q^{2}}= & \frac{\alpha}{2 \pi} P_{\gamma \leftarrow \gamma} \otimes f_{\gamma / \gamma}\left(Q^{2}\right)+\frac{\alpha}{2 \pi} \\
& \times\left[P_{\gamma \leftarrow q} \otimes f_{q / \gamma}\left(Q^{2}\right)+P_{\gamma \leftarrow g} \otimes f_{g / \gamma}\left(Q^{2}\right)\right] .
\end{aligned}
$$

These evolution equations differ from the well-known hadronic case by the inhomogeneous terms of $\mathcal{O}(\alpha)$, which arise from the pointlike coupling of photons to quarks. Equations (3.5) are given for a single quark flavor $q$, but $2 N_{f}$ light quarks and antiquarks are easily accommodated by summing over the index $q$ from one to $2 N_{f}$ whenever it appears twice. The renormalization scale $\mu$ and the factorization scale $M_{f}$ have been identified with the physical scale $Q$. The $x$-space convolution $P_{j \leftarrow i} \otimes f_{i / \gamma}\left(Q^{2}\right)$ reduces to a simple product $P_{j \leftarrow i}(n) f_{i / \gamma}\left(n, Q^{2}\right)$ in Mellin $n$ space, where the $n$th moment is defined as $f(n)=\int_{0}^{1} d x x^{n-1} f(x)$. In moment space, analytical solutions to the evolution equations can be found, but they have to be transformed back to $x$ space for physical cross-section predictions. Alternatively, the evolution equations can be solved directly in $x$ space by iteration, but this requires a careful separation of leading-order and NLO terms in order to avoid spurious higher-order terms. An original ansatz by Rossi (1984) was subsequently generalized by Da Luz Vieira and Storrow (1991) in order to allow for nonzero input parton densities.

The leading-order and NLO splitting functions $P_{j \leftarrow i}$ have been calculated by Curci, Furmanski, and Petronzio (1980) for the flavor-nonsinglet case, by Furmanski and Petronzio (1980) for the coupled gluon and flavor-singlet cases, and also by Floratos, Kounnas, and
Lacaze (1981). Here we review only the leading-order results (Altarelli and Parisi, 1977):

$$
\begin{aligned}
P_{q \leftarrow q}(x)= & C_{F}\left[\frac{1+x^{2}}{(1-x)_{+}}+\frac{3}{2} \delta(1-x)\right]+\mathcal{O}\left(\alpha_{s}\right), \\
P_{g \leftarrow q}(x)= & C_{F}\left[\frac{1+(1-x)^{2}}{x}\right]+\mathcal{O}\left(\alpha_{s}\right), \\
P_{q \leftarrow g}(x)= & T_{R}\left[x^{2}+(1-x)^{2}\right]+\mathcal{O}\left(\alpha_{s}\right), \\
P_{g \leftarrow g}(x)= & 2 N_{C}\left[\frac{1}{(1-x)_{+}}+\frac{1}{x}+x(1-x)-2\right] \\
& +\left[\frac{11}{6} N_{C}-\frac{1}{3} N_{f}\right] \delta(1-x)+\mathcal{O}\left(\alpha_{s}\right),
\end{aligned}
$$

where the + distributions are defined as usual with a test function $f(x)$ in the integral

$$
\int_{0}^{1} d x f(x) g_{+}(x)=\int_{0}^{1} d x[f(x)-f(1)] g(x) .
$$

The photon-quark splitting function can be obtained in leading order from $P_{q \leftarrow g}$ by the transformation $P_{q \leftarrow \gamma}$ $=2 N_{C} e_{q}^{2} P_{q \leftarrow g} . C_{F}=\left(N_{C}^{2}-1\right) /\left(2 N_{C}\right)$ and $N_{C}=3$ are the SU(3) color factors, $T_{R}=1 / 2, N_{f}$ is the number of active flavors, and $e_{q}$ is the fractional quark charge. Since there is no direct coupling of photons to gluons, the photon-gluon splitting function enters only in nextto-leading order. It can be obtained from the NLO gluon-gluon splitting function by replacing the appropriate color factors and dropping the part proportional to $\delta(1-x)$ (Fontannaz and Pilon, 1992):

$$
\begin{aligned}
P_{g \leftarrow \gamma}(x)= & \frac{\alpha_{s}\left(Q^{2}\right)}{2 \pi} \frac{e_{q}^{2} N_{C} C_{F}}{2}\left[-16+8 x+\frac{20}{3} x^{2}+\frac{4}{3 x}\right. \\
& \left.-(6+10 x) \ln x-2(1+x) \ln ^{2} x\right] .
\end{aligned}
$$

\section{B. Boundary conditions and factorization schemes}

The general solutions of the evolution equations (3.5) are given by the sums of pointlike ("anomalous") and hadronic contributions $f_{i / \gamma}\left(Q^{2}\right)=f_{i / \gamma}^{\mathrm{pl}}\left(Q^{2}\right)+f_{i / \gamma}^{\text {had }}\left(Q^{2}\right)$. Due to the pointlike coupling of the photon to quarks, the former can be calculated perturbatively at asymptotically large scales $Q^{2}$ and large Bjorken $x$ in leading order (Witten, 1977) and next-to-leading order (Bardeen and Buras, 1979). The pointlike solution is of the form

$$
f_{i / \gamma}^{\mathrm{pl}}\left(Q^{2}\right)=\frac{\alpha}{2 \pi}\left[\frac{4 \pi}{\alpha_{s}\left(Q^{2}\right)} a_{i}+b_{i}+\mathcal{O}\left(\alpha_{s}\right)\right],
$$

where $a_{i}$ and $b_{i}$ are known analytic functions in moment space. Scaling is already violated at leading order, $\mathcal{O}\left[\alpha / \alpha_{s}\left(Q^{2}\right)\right]$, and the pointlike solution dominates at large $Q^{2}$. As the parton-parton cross sections are of $\mathcal{O}\left(\alpha_{s}^{2}\right)$, the resolved photon contributions are of the same order $\mathcal{O}\left(\alpha \alpha_{s}\right)$ as the direct photon-parton contributions. The pointlike solutions turn out to be singular at low $x$ and moderate $Q^{2}$ (Duke and Owens, 1980) and 
have to be regularized by boundary conditions $f_{i / \gamma}^{\text {had }}\left(Q_{0}^{2}\right)$ at some low starting scale $Q_{0}^{2}$ (Glück and Reya, 1983).

By combining the parton distribution functions $f_{q / \gamma}\left(Q^{2}\right)$ and $f_{g / \gamma}\left(Q^{2}\right)$ with the appropriate Wilson coefficients (Bardeen et al., 1978),

$$
\begin{aligned}
& C_{q}(x)=C_{F}\left[\frac{1+x^{2}}{1-x}\left(\ln \frac{1-x}{x}-\frac{3}{4}\right)+\frac{1}{4}(9+5 x)\right]_{+}, \\
& C_{g}(x)=T_{R}\left(\left[x^{2}+(1-x)^{2}\right] \ln \frac{1-x}{x}+8 x(1-x)-1\right),
\end{aligned}
$$

and (Bardeen and Buras, 1979)

$$
\begin{aligned}
C_{\gamma}(x) & =2 N_{C} C_{g}(x) \\
& =3\left(\left[x^{2}+(1-x)^{2}\right] \ln \frac{1-x}{x}+8 x(1-x)-1\right),
\end{aligned}
$$

one obtains the NLO photon structure function in the $\overline{\mathrm{MS}}$ scheme

$$
\begin{aligned}
F_{2}^{\gamma}\left(Q^{2}\right)= & \sum_{q} 2 x e_{q}^{2}\left\{f_{q / \gamma}\left(Q^{2}\right)+\frac{\alpha_{s}\left(Q^{2}\right)}{2 \pi}\left[C_{q} \otimes f_{q / \gamma}\left(Q^{2}\right)\right.\right. \\
& \left.\left.+C_{g} \otimes f_{g / \gamma}\left(Q^{2}\right)\right]+\frac{\alpha}{2 \pi} e_{q}^{2} C_{\gamma}\right\} .
\end{aligned}
$$

The factor of 2 arises from the fact that $f_{q / \gamma}\left(Q^{2}\right)$ $=f_{\bar{q} / \gamma}\left(Q^{2}\right)$ due to charge-conjugation invariance. Glück, Reya, and Vogt (1992a) observed that the direct term $C_{\gamma}(x)$ contains a term $\ln (1-x)$, which diverges in the large- $x$ region and is better absorbed in the quark distributions $f_{q / \gamma}^{\mathrm{DIS}}\left(Q^{2}\right)=f_{q / \gamma}^{\overline{\mathrm{MS}}}\left(Q^{2}\right)+\alpha /(2 \pi) e_{q}^{2} C_{\gamma}$. Here $\mathrm{DIS}_{\gamma}$ is a deep-inelastic scattering factorization scheme for unpolarized photons. This also affects the NLO photon splitting functions

$$
\begin{aligned}
& P_{q \leftarrow \gamma}^{\mathrm{DIS}_{\gamma}}=P_{q \leftarrow \gamma}^{\overline{\mathrm{MS}}}-e_{q}^{2} P_{q \leftarrow q} \otimes C_{\gamma}, \\
& P_{g \leftarrow \gamma}^{\mathrm{DIS}}=P_{g \leftarrow \gamma}^{\overline{\mathrm{MS}}}-2 \sum_{q} e_{q}^{2} P_{g \leftarrow q} \otimes C_{\gamma} .
\end{aligned}
$$

The definition of the gluon densities in this new DIS ${ }_{\gamma}$ factorization scheme remains unchanged. Equivalently in the $\overline{\mathrm{MS}}$ scheme one can absorb $C_{\gamma}$ (Gordon and Storrow, 1992a, 1997) or its process-independent part (Aurenche, Fontannaz, and Guillet, 1994a) into pointlike initial quark distributions.

Heavy quarks with mass $m_{h}$ and velocity squared $\beta^{2}$ $=1-4 m_{h}^{2} x /\left[(1-x) Q^{2}\right]$ contribute to the photon structure function through the Bethe-Heitler process $\gamma^{*}\left(Q^{2}\right) \gamma \rightarrow h \bar{h}$ with (Budnev et al., 1974; Hill and Ross, 1979)

$$
\begin{aligned}
F_{2, h}^{\gamma}\left(x, Q^{2}\right)= & 3 x e_{h}^{4} \frac{\alpha}{\pi}\left[\beta\left(8 x(1-x)-1-x(1-x) \frac{4 m_{h}^{2}}{Q^{2}}\right)\right. \\
& +\left(x^{2}+(1-x)^{2}+x(1-3 x) \frac{4 m_{h}^{2}}{Q^{2}}-x^{2} \frac{8 m_{h}^{4}}{Q^{4}}\right) \\
& \left.\times \ln \frac{1+\beta}{1-\beta}\right],
\end{aligned}
$$

if the available hadronic energy squared $W^{2}=Q^{2}(1$ $-x) / x$ is larger than the production threshold $4 m_{h}^{2}$. The NLO corrections to Eq. (3.14) are at most 20\% (Laenen et al., 1994) and are usually neglected by authors of photonic parton distributions, but a resolved contribution from the process $\gamma^{*}\left(Q^{2}\right) g \rightarrow h \bar{h}$,

$$
F_{2, h}^{g}\left(x, Q^{2}\right)=\frac{\alpha_{s}\left(Q^{2}\right)}{2 N_{C} e_{h}^{2} \alpha} F_{2, h}^{\gamma}\left(Q^{2}\right) \otimes f_{g / \gamma}\left(Q^{2}\right),
$$

is sometimes included. Far above threshold, heavy quarks are treated as light flavors with boundary conditions $f_{h / \gamma}\left(m_{h}^{2}\right)=f_{\bar{h} / \gamma}\left(m_{h}^{2}\right)=0$.

\section{Hadronic solutions}

Since the $x$ dependence of the boundary conditions $f_{i / \gamma}\left(x, Q_{0}^{2}\right)$ cannot be calculated in perturbation theory, it is necessary to make theoretical assumptions. Usually one takes a form similar to $f_{i / \gamma}\left(x, Q_{0}^{2}\right)=N x^{\alpha}(1-x)^{\beta}$ and fits the normalization $N$ and the exponents $\alpha$ and $\beta$ to experimental data. However, only one particular combination of photonic parton densities, $F_{2}^{\gamma}\left(x, Q^{2}\right)$, is well constrained by experimental data from PETRA, PEP, TRISTAN, and LEP in the ranges $0.001 \leqslant x \leqslant 0.9$ and $0.24 \leqslant Q^{2} \leqslant 390 \mathrm{GeV}^{2}$ (Nisius, 2000), and this combination is dominated by the up-quark density. Thus one has to rely further on models like the vector-mesondominance model (see the review by Bauer et al., 1978), which relates the photon to the $\rho, \omega$, and $\phi$ mesons with $J^{P C}=1^{--}$,

$$
\begin{aligned}
|\gamma\rangle= & \sum_{V=\rho, \omega, \phi} \frac{e}{f_{V}}|V\rangle \\
= & \sqrt{\frac{e^{2}}{f_{\rho}^{2}}+\frac{e^{2}}{f_{\omega}^{2}}\left(e_{u}^{2}+e_{d}^{2}\right)^{-1 / 2}} \\
& \times\left(e_{u}|u \bar{u}\rangle+e_{d}|d \bar{d}\rangle\right)+\frac{e}{f_{\phi}}|s \bar{s}\rangle,
\end{aligned}
$$

and allows for a successful phenomenological description of experimental data on the photoproduction of vector mesons. A flavor SU(3)-symmetric superposition requires $f_{\rho} / f_{\omega}=1 / 3$ and $f_{\rho} / f_{\phi}=-\sqrt{2} / 3$ in fairly good agreement with experimental measurements $(1 / 3.4$ and $-\sqrt{1.3} / 3$; see Table III). A coherent superposition of upand down-quarks, which is favorable at large scales, is obtained with $e_{u}=2 / 3$ and $e_{d}=-1 / 3$, while an incoherent superposition is obtained with $e_{u}=e_{d}=1$.

For safely high starting scales $Q_{0}^{2} \geqslant 2 \mathrm{GeV}^{2}$, the pure VMD ansatz turns out to be insufficient to describe the 
TABLE III. Masses, leptonic decay widths, and decay constants in the zero-width approximation $\Gamma_{e^{+} e^{-}}^{V}=4 \pi \alpha^{2} m_{V} /\left(3 f_{V}^{2}\right)$ for the $\rho, \omega$, and $\phi$ vector mesons (Groom et al., 2000).

\begin{tabular}{cccc}
\hline \hline Vector meson $V$ & $m_{V} / \mathrm{MeV}$ & $\Gamma_{e^{+} e^{-}}^{V} / \mathrm{keV}$ & $f_{V}^{2} /(4 \pi)$ \\
\hline$\rho$ & $769.3 \pm 0.8$ & $6.77 \pm 0.32$ & 2.02 \\
$\omega$ & $782.57 \pm 0.12$ & $0.60 \pm 0.02$ & 23.2 \\
$\phi$ & $1019.417 \pm 0.014$ & $1.297 \pm 0.04$ & 13.96 \\
\hline \hline
\end{tabular}

$F_{2}^{\gamma}$ data at larger $Q^{2}$ and has to be supplemented by an additional hard component. For quarks it can be naturally provided by the quark-box diagram with four external photons, but this is unfortunately not viable for the gluon. Two solutions are possible. The first option is to retain a relatively large $Q_{0}, Q_{0} \geqslant 1 \mathrm{GeV}$, fit the quark densities to $F_{2}^{\gamma}$ data, and estimate the gluon input [Drees and Grassie, 1985; Abramowicz, Charchula, and Levy (LAC), 1991; Gordon and Storrow (GS, GS96), 1992a, 1997; Hagiwara et al. (WHIT), 1995; Schuler and Sjöstrand (SaS 2), 1995; Abramowicz, Gurvich, and Levy, 1998]. The second option is to retain the pure VMD ansatz and start the evolution at a very low scale $Q_{0}$ $\simeq 0.5-0.7 \mathrm{GeV}$ [Aurenche, Chiapetta, et al. (ACFGP), 1992; Glück, Reya, and Vogt (GRV), 1992b; Aurenche, Fontannaz, and Guillet (AFG), 1994a; Schuler and Sjöstrand (SaS 1), 1995; Glück, Reya, and Schienbein (GRSc), 1999b]. See Table IV. Unfortunately the parton distributions in the vector mesons are unknown, so that in practice one has to resort to those in the pseudoscalar pion. The various parametrizations are summarized in Table V.

Recently an energy-momentum sum rule for the photon has been derived (Schuler and Sjöstrand, 1995; Frankfurt and Gurvich, 1996)

$$
\int_{0}^{1} d x x\left[\Sigma\left(x, Q^{2}\right)+f_{g / \gamma}\left(x, Q^{2}\right)+f_{\gamma / \gamma}\left(x, Q^{2}\right)\right]=1,
$$

which further constrains the boundary conditions. $\Sigma\left(x, Q^{2}\right)=\Sigma_{i=1}^{N_{f}} 2 f_{q / \gamma}\left(x, Q^{2}\right)$ is the singlet quark distri- bution. Integrating the third evolution equation in Eq. (3.5), one obtains the solution

$$
\begin{aligned}
& f_{\gamma / \gamma}\left(x, Q^{2}\right) \\
& \quad=\delta(1-x)\left[1-\frac{\alpha}{6 \pi}\left(\sum_{q} 2 N_{C} e_{q}^{2} \ln \frac{Q^{2}}{Q_{0}^{2}}+c\right)\right],
\end{aligned}
$$

which can be directly inserted in Eq. (3.17) with the result

$$
\begin{aligned}
\int_{0}^{1} d x x\left[\Sigma\left(x, Q^{2}\right)+f_{g / \gamma}\left(x, Q^{2}\right)\right] \\
=\frac{\alpha}{6 \pi}\left(\sum_{q} 2 N_{C} e_{q}^{2} \ln \frac{Q^{2}}{Q_{0}^{2}}+c\right) .
\end{aligned}
$$

Hence the quark and gluon momentum fractions rise logarithmically with $Q^{2}$. For the sum rule to be of practical use, the unknown integration constant $c$ has to be related, for example, to the total hadronic cross section in $e^{+} e^{-}$annihilation via a dispersion relation in the photon virtuality. Schuler and Sjöstrand (1995) obtained $c / \pi=\Sigma_{V=\rho, \omega, \phi} 4 \pi / f_{V}^{2} \simeq 0.55$ at $Q_{0}^{2} \simeq 0.36 \mathrm{GeV}^{2}$, while the numerical values in Table III give a slightly larger value of $c / \pi \simeq 0.61$.

Different leading-order and NLO parametrizations of the parton densities in the photon are compared in Fig. 2 at a value of $Q^{2}=25 \mathrm{GeV}^{2}$ relevant for many hard photoproduction processes. In leading order (left) the $\mathcal{O}\left(\alpha, \alpha_{s}\right)$ terms in Eq. (3.12) do not contribute and $F_{2}^{\gamma}\left(x, Q^{2}\right)$ is directly related to the quark densities. Thus the experimental constraints on $F_{2}^{\gamma}\left(x, Q^{2}\right)$ lead to fairly good agreement of the different quark density param-

TABLE IV. Parametrizations of photon densities and photon fragmentation functions.

\begin{tabular}{ll}
\hline \hline Parametrization & \multicolumn{1}{c}{ Reference } \\
\hline ACFGP & Aurenche, Chiapetta, et al. (1992, 1993) \\
AFG & Aurenche, Fontannaz, and Guillet (1994a) \\
BFG & Bourhis, Fontannaz, and Guillet (1998) \\
DG & Drees and Grassie (1985) \\
GAL & Abramowicz, Gurvich, and Levy (1998) \\
GRSc & Glück, Reya, and Schienbein (1999b, 2001) \\
GRSi & Glück, Reya, and Sieg (2001a, 2001b) \\
GRSt & Glück, Reya, and Stratmann (1995, 1996) \\
GRV & Glück, Reya, and Vogt (1992b, 1993) \\
GStV & Glück, Stratmann, and Vogelsang (1994) \\
GS & Gordon and Storrow (1992a, 1997) \\
LAC & Abramowicz, Charchula, and Levy (1991) \\
SaS & Schuler and Sjöstrand (1995) \\
WHIT & Hagiwara et al. (1995) \\
\hline \hline
\end{tabular}


TABLE V. Parametrizations of the parton densities in the photon. The sets SaS $1 \mathrm{M}$ and $2 \mathrm{M}$ are defined by absorbing the $C_{\gamma}$ coefficient in Eq. (3.11) into the leading-order quark distributions. The coherence of the vector-meson-dominance (VMD) model is determined by the coefficients $e_{u}$ and $e_{d}$ in Eq. (3.16). Most parametrizations fit $F_{2}^{\gamma}$ (except ACFGP, AFG, and GRSc) and the pion densities (except DG, LAC, WHIT, SaS, and GAL) and add the direct Bethe-Heitler contribution for heavy quarks in Eq. (3.14) (except DG, LAC, and GAL). WHIT, SaS, and GRSc also add the resolved contribution in Eq. (3.15).

\begin{tabular}{|c|c|c|c|c|c|c|c|}
\hline Group & Year & Set & $\begin{array}{c}Q_{0}^{2} \\
\left(\mathrm{GeV}^{2}\right) \\
\end{array}$ & $\begin{array}{l}\text { Factor. } \\
\text { scheme }\end{array}$ & $\begin{array}{l}\text { VMD } \\
\text { model }\end{array}$ & $N_{f}$ & $\begin{array}{l}\Lambda_{\overline{\mathrm{MS}}}^{N_{f}=4} \\
(\mathrm{MeV})\end{array}$ \\
\hline $\mathrm{DG}$ & 1985 & $1-3$ & 1 & $\mathrm{LO}$ & & $3-5$ & 400 \\
\hline LAC & 1991 & $1-3$ & $4,4,1$ & $\mathrm{LO}$ & & 4 & 200 \\
\hline \multirow[t]{2}{*}{ GS } & 1992 & $\mathrm{LO}$ & 5.30 & $\mathrm{LO}$ & incoherent & 5 & 200 \\
\hline & & NLO & 5.30 & $\overline{\mathrm{MS}}$ & incoherent & 5 & 200 \\
\hline \multirow[t]{2}{*}{ GRV } & 1992 & $\mathrm{LO}$ & 0.25 & $\mathrm{LO}$ & incoherent & 5 & 200 \\
\hline & & NLO & 0.30 & $\mathrm{DIS}_{\gamma}$ & incoherent & 5 & 200 \\
\hline ACFGP & 1992 & NLO & 0.25 & $\overline{\mathrm{MS}}$ & coherent & 4 & 200 \\
\hline AFG & 1994 & NLO & 0.50 & $\overline{\mathrm{MS}}$ & coherent & 4 & 200 \\
\hline WHIT & 1995 & $1-6$ & 4.00 & $\mathrm{LO}$ & & 4 & 400 \\
\hline \multirow[t]{2}{*}{$\mathrm{SaS}$} & 1995 & $1 \mathrm{D} / \mathrm{M}$ & 0.36 & $\mathrm{LO}$ & coherent & 5 & 200 \\
\hline & & $2 \mathrm{D} / \mathrm{M}$ & 4.00 & $\mathrm{LO}$ & coherent & 5 & 200 \\
\hline \multirow[t]{2}{*}{ GS96 } & 1997 & $\mathrm{LO}$ & 3.00 & $\mathrm{LO}$ & incoherent & 5 & 200 \\
\hline & & NLO & 3.00 & $\overline{\mathrm{MS}}$ & incoherent & 5 & 200 \\
\hline GAL & 1998 & $\mathrm{LO}$ & 4 & $\mathrm{LO}$ & & 4 & 200 \\
\hline \multirow[t]{2}{*}{ GRSc } & 1999 & $\mathrm{LO}$ & 0.26 & $\mathrm{LO}$ & coherent & 3 & 204 \\
\hline & & NLO & 0.40 & $\mathrm{DIS}_{\gamma}$ & coherent & 3 & 299 \\
\hline
\end{tabular}

etrizations, particularly at large $x(x>0.1)$. Below this value, the WHIT 6 and also the similar WHIT 2-5 quark densities show a steep rise, which is caused by a similar rise of the gluon density. While this behavior is consistent with the large- $x$ data used in the fits, it is strongly disfavored by recent small- $x$ data from LEP (Nisius, 2000). The parametrizations with a high starting scale (WHIT, SaS 2, and GS96) have larger quark densities at large $x$ than those with a low starting scale (GRSc and $\mathrm{SaS} 1$ ). The sets SaS $1 \mathrm{M}$ and SaS $2 \mathrm{M}$, defined by absorbing $C_{\gamma}$ into pointlike initial quark distributions even in leading order, turn out to be similar to the sets SaS 1D and SaS $2 \mathrm{D}$ after subtracting the $C_{\gamma}$ term. If the vectormeson states are added in a coherent fashion (GRSc and $\mathrm{SaS}$ ), the $d$-quark input density in the photon is suppressed by a factor of 4 with respect to the $u$-quark density. In this case the low- $x$ behaviors differ, and the momentum sum rule Eq. (3.19) can be fulfilled. With an incoherent superposition, the $u$-quark and $d$-quark densities are identical at low $x$ (GS96 and WHIT), and the momentum sum rule is easily violated. The gluon density does not enter directly in Eq. (3.12) in leading order. It enters only through a rather weak coupling to the singlet in the evolution equations and is consequently badly constrained. Only a very steep rise at low $x$ (LAC 1-2, WHIT 2-6) and a very hard gluon (LAC 3) can be ruled out by $F_{2}^{\gamma}$ data from LEP and jet or particle production data from HERA. On the other hand, the HERA data indicate that the SaS 1D and the almost identical GRSc gluon densities may be too low (Nisius, 2000).
The NLO parton densities are shown on the righthand side of Fig. 2 in the DIS $\gamma$ scheme. The AFG and GS96 quark densities have been properly transformed, while the GRSc and GRV densities are given directly in

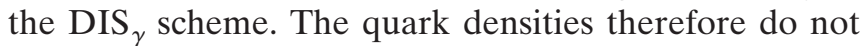
exhibit the $\overline{\mathrm{MS}}$ singularity at $x=1$, but have shapes similar to their leading-order counterparts. Furthermore, the GRSc and AFG densities are very similar, since both groups use a coherent VMD ansatz at a low starting scale and relate the $\rho$-meson input to recent determinations of the pion densities. An incoherent superposition and older pion parametrizations have been used in the GRV distributions, resulting in a steeper rise for the identical $u$-quark and $d$-quark sea distributions at small $x$. The gluon distribution enters directly in $F_{2}^{\gamma}$ in NLO [see Eq. (3.12)] and can therefore be better constrained. Again the GRV distribution exhibits a steeper shape than GRSc and AFG, and the GS96 result is substantially harder. By definition the NLO GS96 set should, like the older NLO GS set, lead to the same $F_{2}^{\gamma}\left(x, Q_{0}^{2}\right)$ as in leading order. However, the NLO quark parametrizations turn out to be too small over the full $x$ range. The leading-order $F_{2}^{\gamma}\left(x, Q_{0}^{2}\right)$ results and the figures in the original papers (Gordon and Storrow, 1992a, 1997) cannot be reproduced with the available NLO parametrization, which is therefore not usable in its present form.

The three usable NLO parametrizations of the photon structure function (GRSc, AFG, and GRV) are confronted with the world data on $F_{2}^{\gamma}\left(x, Q^{2}\right)$ in Figs. 3 


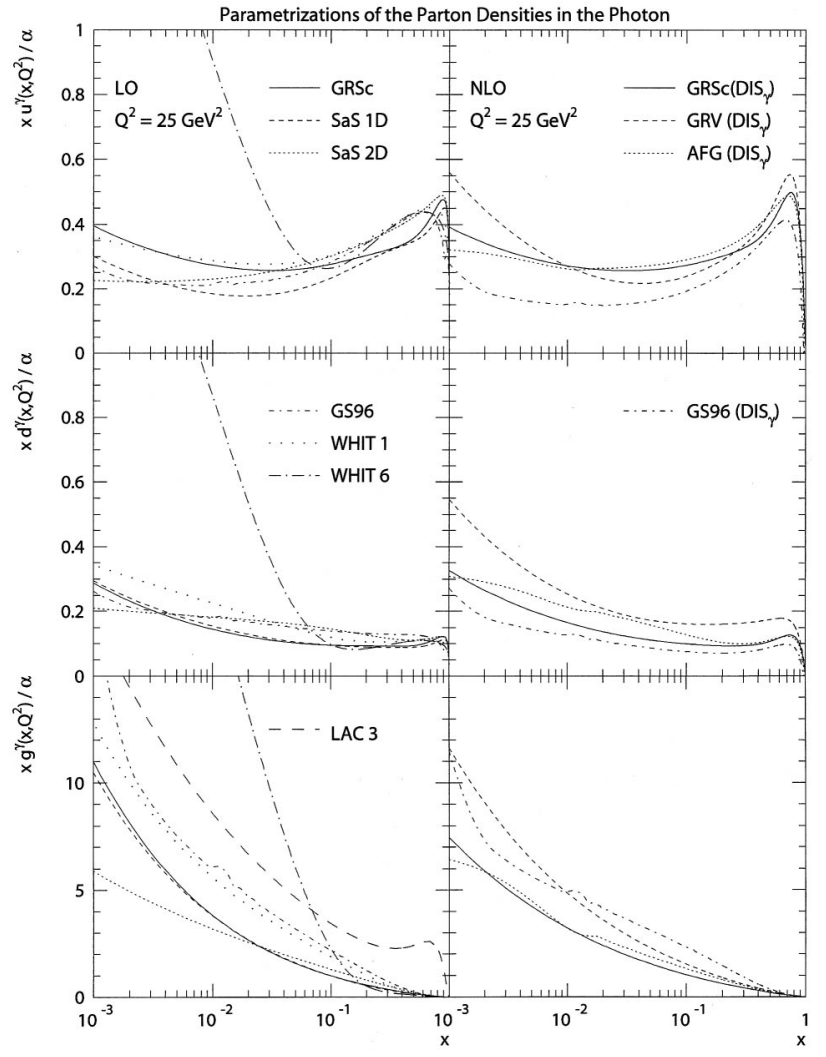

FIG. 2. Parametrizations of the up-quark (top), down-quark (center), and gluon (bottom) densities in the photon in leading order (LO; left) and next-to-leading order (NLO; right) at $Q^{2}=25 \mathrm{GeV}^{2}$. The next-to-leading-order AFG and GS96 (Aurenche, Fontannaz, and Guillet, 1994a; Gordon and Storrow, 1997) parametrizations have been transformed from the $\overline{\mathrm{MS}}$ to the $\mathrm{DIS}_{\gamma}$ scheme.

(small $x$ ) and 4 (large $x)$. None of the parametrizations dare to describe the TPC $/ 2 \gamma$ data at $Q^{2}=0.24 \mathrm{GeV}^{2}$, which may be too close to $\Lambda^{2} \simeq 0.04-0.16 \mathrm{GeV}^{2}$ to allow for a perturbative treatment (see Fig. 3). The first NLO parametrization (GRV) starts at $Q_{0}^{2}=0.30 \mathrm{GeV}^{2}$. While it is still lower than the $\mathrm{TPC} / 2 \gamma$ data at $Q^{2}$ $=0.38 \mathrm{GeV}^{2}$, it fits the TPC/2 $\gamma$ data rather well at $Q^{2}$ $=0.7$ and $1.3 \mathrm{GeV}^{2}$. The low- $x$ and low- $Q^{2}$ data from OPAL and L3 are slightly underestimated. The GRSc parametrization is evolved from $Q_{0}^{2}=0.40 \mathrm{GeV}^{2}$. It describes the LEP data at small $x$ best, due to the most recent determination of the pion structure. AFG starts at $Q_{0}^{2}=0.50 \mathrm{GeV}^{2}$, but this parametrization should only be compared to data above $Q^{2} \simeq 2 \mathrm{GeV}^{2}$. The theoretical assumptions are very similar to those of GRSc, and so are the results for $F_{2}^{\gamma}$, AFG being generally slightly larger. At larger $x$ and $Q^{2}$ (Fig. 4), the hadronic contribution decreases, while the pointlike contribution increases. Consequently the different parametrizations converge, and they all describe the data very well. The factorization scheme starts to play a role at very large $x$, and thus the AFG $\overline{\mathrm{MS}}$ prediction differs strongly from the GRSc and GRV DIS ${ }_{\gamma}$ predictions. The GRSc charm contribution is provided only by the direct and resolved Bethe-Heitler terms in Eqs. (3.14) and (3.15) for $Q^{2}(1$

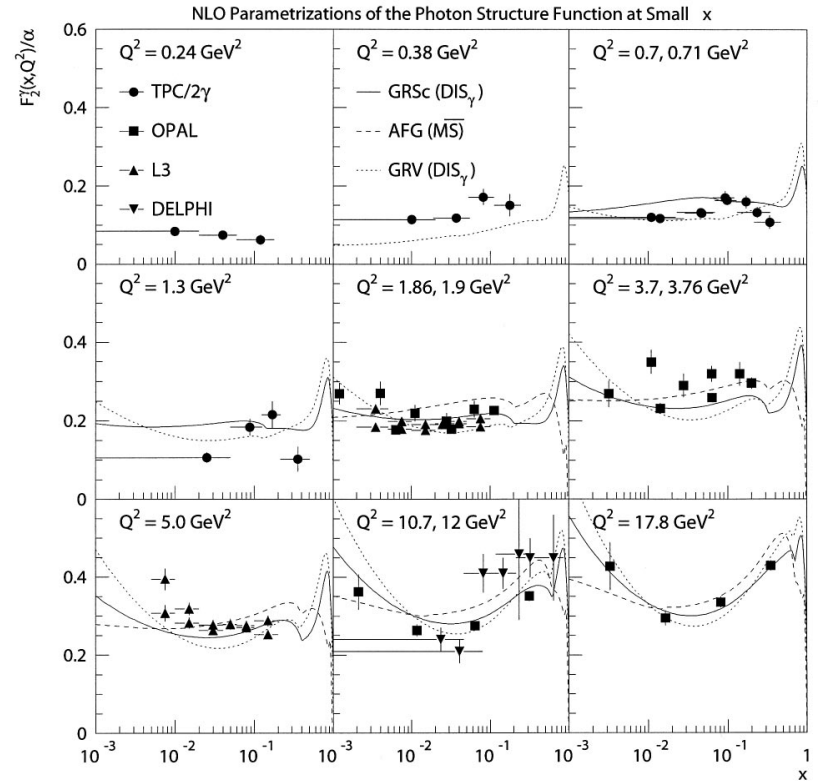

FIG. 3. Next-to-leading-order parametrizations of the photon structure function at small $x$ compared to the world data on $F_{2}^{\gamma}$ (Nisius, 2000). The GRSc (Glück et al., 1999b) and GRV (Glück et al., 1992b) parametrizations are given in the DIS scheme, while the AFG (Aurenche, Fontannaz, and Guillet, 1994a) parametrization is in the $\overline{\mathrm{MS}}$ scheme.

$-x) / x>4 m_{c}^{2}$, while GRV and AFG have included the charm quark also in the massless evolution Eqs. (3.5). GRSc and AFG choose $m_{c} \simeq 1.4 \mathrm{GeV}$, while GRV take $m_{c}=1.5 \mathrm{GeV}$, so that the charm contribution sets on at slightly different values of $x$.

Early hopes of extracting the strong-coupling constant from the photon structure function (Witten, 1977) were dashed by complications arising in higher orders (Bardeen and Buras, 1979; Antoniadis and Grunberg, 1983; Glück and Reya, 1983; Rossi, 1983; Field, Kapusta, and Poggioli, 1987). Since then it has been widely believed that the sensitivity of the photon structure function to the strong coupling is low. Most of the parametrizations use an approximate NLO solution of the renormalization-group equation,

$$
\alpha_{s}\left(Q^{2}\right)=\frac{4 \pi}{\beta_{0} \ln \left(Q^{2} / \Lambda^{2}\right)}-\frac{4 \pi \beta_{1}}{\beta_{0}^{3}} \frac{\ln \ln \left(Q^{2} / \Lambda^{2}\right)}{\left[\ln \left(Q^{2} / \Lambda^{2}\right)\right]^{2}},
$$

with the one- and two-loop $\beta$ functions $\beta_{0}=11-2 N_{f} / 3$ and $\beta_{1}=102-38 N_{f} / 3$ and the fundamental QCD scale parameter $\Lambda_{\overline{\mathrm{MS}}}^{N_{f}=4}=200 \mathrm{MeV}$. GS use the same $\Lambda$ value for all $N_{f}$, while GRSc take $\alpha_{s}\left(M_{Z}\right)=0.114$ and solve the renormalization-group equation numerically. However, a new analysis shows that the now final PETRA, TRISTAN, and LEP data lead to a competitive determination of $\alpha_{s}$ from a single-parameter pointlike fit at large $x$ and $Q^{2}$ with $\alpha_{s}\left(m_{Z}\right)=0.1183$ \pm 0.0050 (exp. $)_{-0.0028}^{+0.0029}$ (theor.) and from a five-parameter 


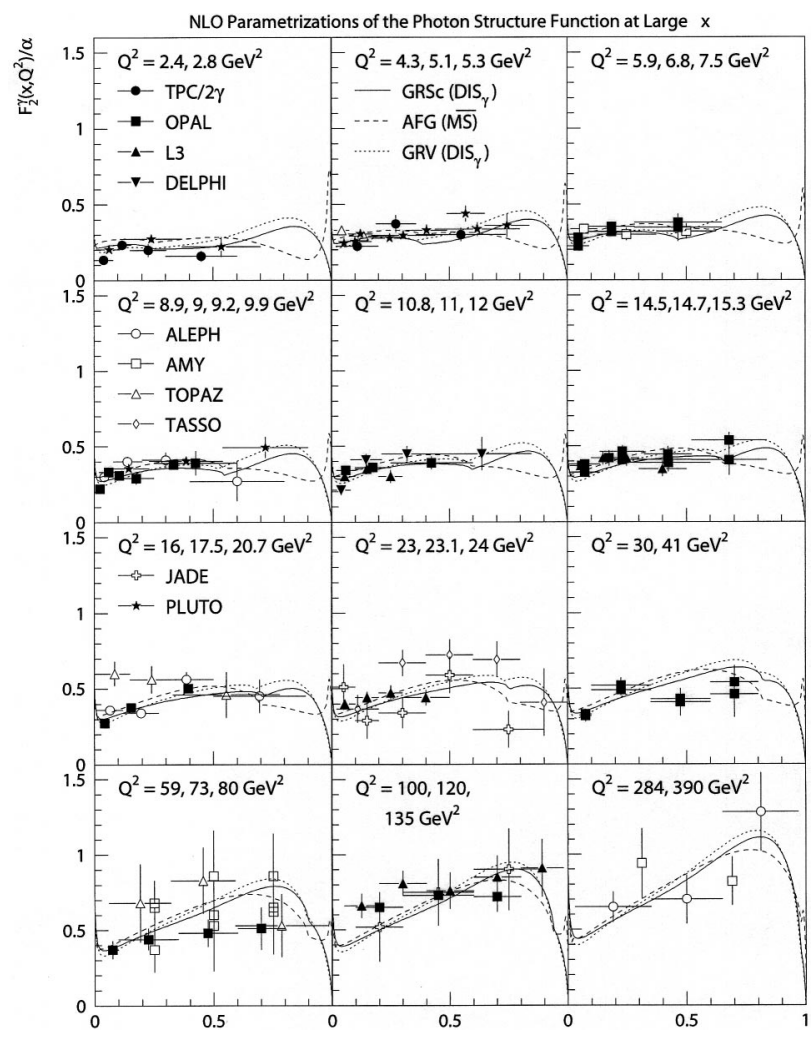

FIG. 4. Same as Fig. 3, but now for large $x$.

full (pointlike and hadronic) fit at all $x$ and $Q^{2}$ with $\alpha_{s}\left(m_{Z}\right)=0.1198 \pm 0.0028$ (expt.) ${ }_{-0.0046}^{+0.0034}$ (theor.) (Albino, Klasen, and Söldner-Rembold, 2002).

The hadronic structure of the photon is currently much less well known than that of the proton, and current parametrizations rest largely on similar theoretical assumptions like vector-meson dominance. To improve the situation, the light-quark and gluon densities have to be disentangled from various observables in jet, hadron, and prompt-photon production (see the following sections), and the heavy-quark contributions have to be consistently resummed according to variable flavor number schemes.

\section{JET PRODUCTION}

Due to the confinement properties of QCD, only hadrons, and not partons, are observable as asymptotic states. The hadronization process happens at low scales, where the coupling $\alpha_{s}$ is large, and is therefore not calculable in perturbation theory. It can, however, be modeled by combining several partons or hadrons moving in the same direction into jets. At sufficiently large transverse energies, the production cross section can then be calculated perturbatively and jets can be related to partons.

Jets were first observed in event deformations at the $e^{+} e^{-}$colliders SPEAR (Hanson et al., 1975) and DORIS (Berger et al., 1978), where they helped to identify the quark spin. At PETRA they could be seen even by the naked eye. They established the existence of the gluon (Bartel et al., 1980; Berger et al., 1980; Brandelik et al., 1980; Behrend et al., 1982) and were also observed in photon-photon collisions (Bartel et al., 1981; Brandelik et al., 1981; Althoff et al., 1984; Berger et al., 1984a, 1985, 1987; Behrend et al., 1991). Jet production in photon-hadron collisions was subsequently observed at Fermilab (Adams et al., 1994) and at HERA (Abt et al., 1993; Derrick et al., 1995a). In the following, various jet definitions and jet cross sections will be discussed.

\section{A. Cone and cluster algorithms}

In Sec. III we showed how photons can split into massless quark-antiquark pairs and exhibit a collinear singularity. Collinear divergences arise quite generally in scattering processes whenever a massless parton splits into two. In addition, infrared divergences are generated by the emission of soft particles. Within the context of QED, it could be proven that soft (Bloch and Nordsieck, 1937) and collinear (Kinoshita, 1962) divergences cancel between real and virtual emission in total cross sections. Lee and Nauenberg (1964) demonstrated that the cancellation also happens for differential cross sections if they are summed over degenerate initial and final states. In QED the collinear divergence is regulated by the electron mass, but in QCD it occurs even for nonzero quark masses, since one massless gluon can split into two. Nevertheless, infrared and collinear singularities are expected to cancel for all leading-twist QCD observables. A rigorous proof of the Kinoshita-Lee-Nauenberg theorem does not exist for QCD, but in practical applications counterexamples have never been found.

Shortly after the first experimental observation of jets, Sterman and Weinberg (1977) realized that beyond leading order there can be no one-to-one correspondence between jets and partons, but that jet definitions must be infrared safe and satisfy the Kinoshita-Lee-Nauenberg theorem order by order in perturbation theory. In practice, jet definitions should be simple to implement in experimental analyses and theoretical calculations and should be insensitive to hadronization effects. In the Sterman-Weinberg definition, a final state in $e^{+} e^{-}$annihilation is classified as two-jet-like if all but an energy $\epsilon$ is contained in a pair of cones of half-angle $\delta$, while all other events are classified as three-jet-like. This cone definition is infrared safe, but it is not well suited for events with more than three jets, and it separates the phase space in an inefficient way. A better solution is provided by clustering algorithms such as the one used by the JADE Collaboration (Bethke et al., 1988), in which two particles $i$ and $j$ are combined into a jet and their four-momenta $p_{i, j}$ are added, if their invariant mass

$$
\left(p_{i}+p_{j}\right)^{2}=2 E_{i} E_{j}\left(1-\cos \theta_{i j}\right)<y S
$$

is smaller than some fixed fraction $y$ of the overall center-of-mass energy $S$. Theoretically the JADE algorithm is not well suited to resum logarithms of 
$\mathcal{O}\left(\alpha_{s}^{n} \ln ^{2 n} y\right)$, which can become large for small values of $y$. This is easier in the $k_{T}$ or Durham algorithm (Catani et al., 1991), in which two particles are combined if their energies $E_{i, j}$ and the angle $\theta_{i j}$ between them satisfy the condition

$$
2 \min \left(E_{i}^{2}, E_{j}^{2}\right)\left(1-\cos \theta_{i j}\right)<y S .
$$

In photon and hadron collisions, the partonic system is boosted along the beam axis, and the remnants of the beams must be separated from the hard jets. It is therefore convenient to define outgoing particles $i$ in the transverse plane with four-momenta

$$
\begin{aligned}
p_{i} & =\left(E_{i}, p_{x_{i}}, p_{y_{i}}, p_{z_{i}}\right) \\
& =\left(m_{T_{i}} \cosh y_{i}, p_{T_{i}} \cos \phi_{i}, p_{T_{i}} \sin \phi_{i}, m_{T_{i}} \sinh y_{i}\right),
\end{aligned}
$$

transverse masses $m_{T_{i}}=\sqrt{p_{T_{i}}^{2}+m_{i}^{2}}$, transverse momenta $p_{T_{i}}$, azimuthal angles $\phi_{i}$, and rapidities

$$
y_{i}=\frac{1}{2} \ln \left(\frac{E_{i}+p_{z_{i}}}{E_{i}-p_{z_{i}}}\right) .
$$

Experimentally the jet energies $E_{i}$ and scattering angles $\theta_{i}$ are measured, and the $p_{T_{i}}$ are replaced with $E_{T_{i}}$ $=E_{i} \sin \theta_{i}$. The rapidities $y_{i}$ are additive under boosts along the $z$ direction. For massless particles they coincide with the pseudorapidities $\eta_{i}=-\ln \left[\tan \left(\theta_{i} / 2\right)\right]$. According to the standardization of the 1990 Snowmass meeting (Huth et al., 1990), particles $i$ are added to a jet cone $J$ if they have a distance

$$
R_{i}=\sqrt{\left(\eta_{i}-\eta_{J}\right)^{2}+\left(\phi_{i}-\phi_{J}\right)^{2}}<R
$$

from the cone center. $R=0.7-1$ is the jet radius in the $\eta-\phi$ plane and

$$
\begin{aligned}
& E_{T_{J}}=\sum_{R_{i}<R} E_{T_{i}}, \quad \eta_{J}=\frac{1}{E_{T_{J}}} \sum_{R_{i}<R} E_{T_{i}} \eta_{i}, \\
& \phi_{J}=\frac{1}{E_{T_{J}}} \sum_{R_{i}<R} E_{T_{i}} \phi_{i}
\end{aligned}
$$

define the jet transverse energy and axis. In NLO QCD two partons, which may be separated by as much as $2 R$, can be combined if they have equal transverse energies. In this case, one could count the individual jets in addition to the combined jet, but this double counting should be avoided (Ellis, Kunszt, and Soper, 1989b). The broad combined jet is difficult to find experimentally, since it does not have a seed in its center. This led Ellis, Kunszt, and Soper (1992) to propose an additional parameter $R_{\text {sep }}$, which restricts the distance

$$
\begin{aligned}
R_{i j} & =\sqrt{\left(\eta_{i}-\eta_{j}\right)^{2}+\left(\phi_{i}-\phi_{j}\right)^{2}} \\
& <\min \left[\frac{E_{T_{i}}+E_{T_{j}}}{\max \left(E_{T_{i}}, E_{T_{j}}\right)} R, R_{\text {sep }}\right]
\end{aligned}
$$

of two partons $i$ and $j$. However, the value of $R_{\text {sep }}$ $=R-2 R$ must be fitted to different experimental imple-

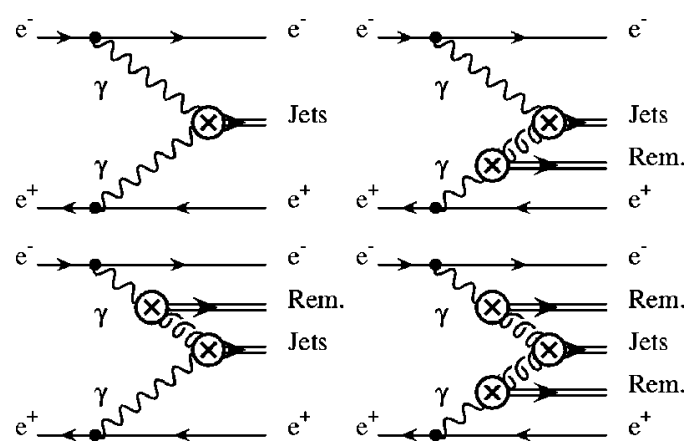

FIG. 5. Factorization of photon-photon scattering into jets.

mentations of the Snowmass algorithm (Butterworth et al., 1996; Klasen and Kramer, 1997b). Furthermore, studies of three-jet hadroproduction (Giele and Kilgore, 1997) and jet shapes (Seymour, 1998) revealed that the Snowmass cone algorithm is not infrared safe in higher orders unless the centers of jet pairs are also considered as seeds (Akers et al., 1994).

The deficiencies of the cone algorithm are remedied in the longitudinally invariant $k_{T}$ clustering algorithm (Catani, Dokshitzer, and Webber, 1992; Catani et al., 1993; Ellis and Soper, 1993), where one uses only the combination criterion $R_{i j}<R_{\text {sep }}$ with the $E_{T}$-weighted recombination scheme as in Eq. (4.6). The results obtained with the cluster algorithm in hadron-hadron collisions become similar to those obtained with the cone algorithm if one chooses $R_{\text {sep }} \simeq 1.35 R$ (Ellis and Soper, 1993). This was also found to be the optimal value of $R_{\text {sep }}$ for the cone algorithm in photoproduction (Butterworth et al., 1996; Klasen and Kramer, 1997b).

\section{B. Single jets}

Since photons couple to charged quarks, they can participate directly in hard scattering processes like jet production. On the other hand, they can also exhibit a partonic structure (see Sec. III) and interact indirectly through their constituents (quarks and gluons). In photon-photon scattering, this leads to the direct, singleresolved, and double-resolved processes shown in Fig. 5, in which the hard central jets are produced in association with zero, one, or two forward-going photon remnant jets. In photon-hadron scattering, the single- and double-resolved photon processes described above correspond to the direct and resolved photon processes in Fig. 6, which involve a proton remnant jet and, for resolved photoproduction, also a photon remnant jet.

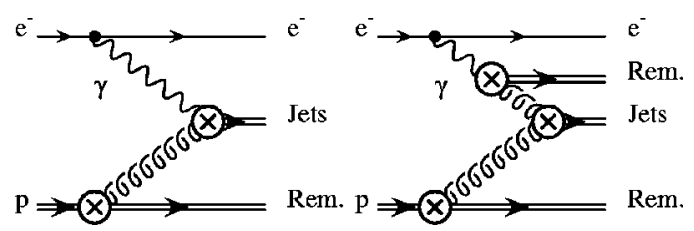

FIG. 6. Factorization of photon-hadron scattering into jets. 


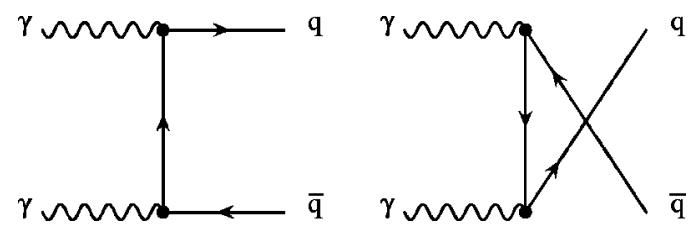

FIG. 7. Born diagrams for direct $\gamma \gamma$ scattering.

The leading-order partonic cross section for the scattering of two massless partons $a$ and $b$ into two massless partons 1 and 2 is

$$
\frac{d \sigma^{B}}{d t}=\frac{1}{2 s} \frac{1}{\Gamma(1-\varepsilon)}\left(\frac{4 \pi s}{t u}\right)^{\varepsilon} \frac{1}{8 \pi s} \frac{g_{a, b}^{2}}{S_{a} S_{b} C_{a} C_{b}}\left|\mathcal{M}^{B}\right|^{2}
$$

in $n=4-2 \varepsilon$ dimensions. $s=\left(p_{a}+p_{b}\right)^{2}, t=\left(p_{a}-p_{1}\right)^{2}$, and $u=\left(p_{a}-p_{2}\right)^{2}$ are the Lorentz-invariant Mandelstam variables, $\Gamma(x)$ is the Euler $\Gamma$ function, and $g_{a, b}^{2}$ are the squared coupling constants $\left[4 \pi \alpha e_{q}^{2}\right.$ for photons and $4 \pi \alpha_{s}\left(\mu^{2}\right)$ for quarks and gluons]. $S_{a, b}$ are the spin degrees of freedom of the initial particles ( 2 for quarks and $n-2$ for photons and gluons) and $C_{a, b}$ are the initial color degrees of freedom ( 1 for photons, $N_{C}$ for quarks, and $N_{C}^{2}-1$ for gluons). While one can safely set $n=4$ or $\varepsilon=0$ in Eq. (4.8) and in the squared matrix element $\left|\mathcal{M}^{B}\right|^{2}$ in leading-order calculations (Brodsky et al., 1978, 1979; Fontannaz et al., 1980; Owens, 1980; Baer, Ohnemus, and Owens, 1989a), the $\mathcal{O}(\varepsilon)$ terms are needed in NLO in connection with ultraviolet, infrared, and collinear divergences (see below). In jet cross sections, the partonic masses $p_{i}^{2}=m_{i}^{2} \ll E_{T}^{2}$ can usually be neglected, and the Mandelstam variables

$$
\begin{aligned}
& s=4 x_{a} x_{b} E_{A} E_{B}, \quad t=-2 x_{a} E_{A} E_{T} e^{-\eta}, \\
& u=-2 x_{b} E_{B} E_{T} e^{\eta}
\end{aligned}
$$

become simple functions of $E_{T}, \eta, E_{A, B}$, and $x_{a, b}$.

The massless Born diagrams for the direct process $\gamma \gamma \rightarrow q \bar{q}$, the single-resolved process $\gamma g \rightarrow q \bar{q}$, and the double-resolved processes $q q^{\prime} \rightarrow q q^{\prime}, \quad q q \rightarrow q q, \quad q \bar{q}$ $\rightarrow g g$, and $g g \rightarrow g g$ are shown in Figs. 7-9, and the corresponding squared matrix elements $\left|\mathcal{M}^{B}\right|^{2}$ are summarized in Table VI. All other Born diagrams and squared matrix elements can be obtained by crossing particle lines and Mandelstam variables and including a factor of $(-1)$ for every crossed fermion line.

In next-to-leading order, the partonic cross section

$$
\frac{d \sigma}{d t}=\frac{d \sigma^{B}}{d t}+\frac{d \sigma^{V}}{d t}+\frac{d \sigma^{F}}{d t}+\frac{d \sigma^{I}}{d t}
$$

receives additional contributions from virtual loop cor-

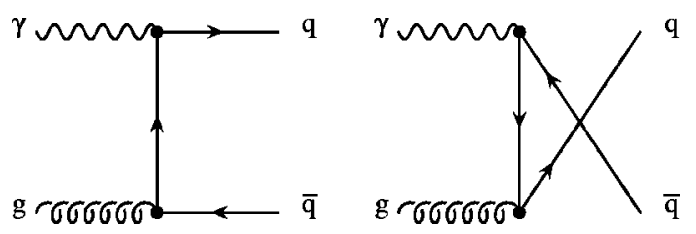

FIG. 8. Born diagrams for single-resolved $\gamma g$ scattering.

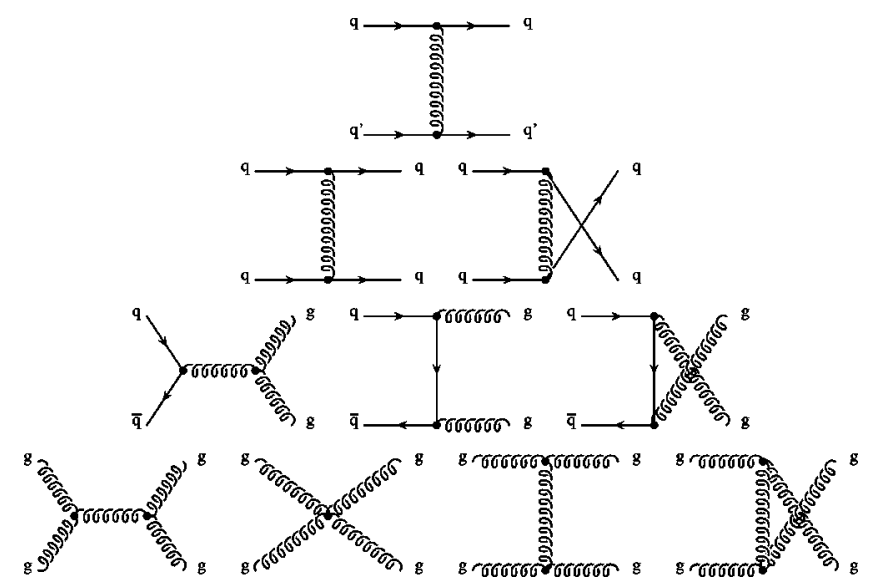

FIG. 9. Born diagrams for double-resolved $q q^{\prime}, q q, q \bar{q}$, and gg scattering.

rections $(V)$ and real emission corrections in the final state $(F)$ and initial state $(I)$ with

$$
\begin{aligned}
\frac{d \sigma^{V, F, I}}{d t}= & \frac{1}{2 s} \frac{1}{\Gamma(1-\varepsilon)}\left(\frac{4 \pi s}{t u}\right)^{\varepsilon} \frac{1}{8 \pi s} \frac{g_{a, b}^{2}}{S_{a} S_{b} C_{a} C_{b}} \\
& \times \frac{\alpha_{s}}{2 \pi}\left(\frac{4 \pi \mu^{2}}{Q^{2}}\right)^{\varepsilon} \frac{\Gamma(1-\varepsilon)}{\Gamma(1-2 \varepsilon)}\left|\mathcal{M}^{V, F, I}\right|^{2} .
\end{aligned}
$$

Virtual corrections arise in next-to-leading order by dressing all constituents of the leading-order diagrams in Figs. 7-9 with one-loop particle exchanges. Figure 10 shows the self-energy diagrams for quarks (top) and gluons (bottom), which are factorizable and thus independent of the scattering process and have to be inserted in external and internal quark and gluon lines. The ghost self-energy is obtained by replacing quark lines with ghost lines in the upper diagram. While photon selfenergies arise also at one loop, they are of $\mathcal{O}(\alpha)$, not of $\mathcal{O}\left(\alpha_{s}\right)$, and thus are not included in NLO QCD calculations. Vertex corrections are shown in Fig. 11 for the quark-gluon (top), triple-gluon (center), and four-gluon vertices (bottom). For the latter two, additional diagrams are generated by permutations of the participating particles. The corrections to the ghost-gluon vertex are of a type similar to those for the quark-gluon vertex, and the photon-quark vertex correction is obtained from the second diagram in the first row of Fig. 11 by replacing the outgoing gluon with a photon. Vertex corrections depend on the four-momentum flow at the vertex and thus factorize only at the amplitude, and not the squared amplitude, level. A third type of loop diagram is generated by box diagrams, which depend on the types and momenta of all parton legs in a $2 \rightarrow 2$ Born process and also factorize only at the amplitude level. Typical examples are the boxes generated by the four-gluon vertex correction in the last row of Fig. 11. All loop diagrams with a closed fermion line receive a factor $(-1)$ and those with identical particles (gluons) a statistical factor of $1 / 2 .\left|\mathcal{M}^{V}\right|^{2}$ in Eq. (4.11) denotes the interference terms of Born and virtual one-loop matrix elements, which have been integrated over the loop momentum $l$ with a measure $d^{n} l /(2 \pi)^{n}$. In this case only the real 
TABLE VI. Leading-order (LO) squared matrix elements $\left|\mathcal{M}^{B}\right|^{2}$ for massless $2 \rightarrow 2$ parton processes involving two, one, and no photons in $n=4-2 \varepsilon$ dimensions.

\begin{tabular}{ll}
\hline \hline Process & LO matrix element squared $\left|\mathcal{M}^{B}\right|^{2}$ \\
\hline$\gamma \gamma \rightarrow q \bar{q}$ & $8 N_{C}(1-\varepsilon)\left[(1-\varepsilon)\left(\frac{u}{t}+\frac{t}{u}\right)-2 \varepsilon\right]$ \\
$\gamma g \rightarrow q \bar{q}$ & $C_{F}\left|\mathcal{M}^{B}\right|_{\gamma \gamma \rightarrow q \bar{q}}^{2}(s, t, u)$ \\
$q q^{\prime} \rightarrow q q^{\prime}$ & $4 N_{C} C_{F}\left(\frac{s^{2}+u^{2}}{t^{2}}-\varepsilon\right)$ \\
$q q \rightarrow q q$ & {$\left[\left|\mathcal{M}^{B}\right|_{q q^{\prime} \rightarrow q q^{\prime}}^{2}(s, t, u)+\left|\mathcal{M}^{B}\right|_{q q^{\prime} \rightarrow q q^{\prime}}^{2}(s, u, t)\right.$} \\
& $\left.-8 C_{F}(1-\varepsilon)\left(\frac{s^{2}}{u t}+\varepsilon\right)\right] / 2 !$ \\
$q \bar{q} \rightarrow g g$ & {$\left[4 C_{F}(1-\varepsilon)\left(\frac{2 N_{C} C_{F}}{u t}-\frac{2 N_{C}^{2}}{s^{2}}\right)\left(t^{2}+u^{2}-\varepsilon s^{2}\right)\right] / 2 !$} \\
$g g \rightarrow g g$ & {$\left[32 N_{C}^{3} C_{F}(1-\varepsilon)^{2}\left(3-\frac{u t}{s^{2}}-\frac{u s}{t^{2}}-\frac{s t}{u^{2}}\right)\right] / 2 !$} \\
\hline \hline
\end{tabular}

parts of the loop integrals need be kept. In loop diagrams with internal fermion lines, the integration momentum $l$ appears not only in the propagator denominator, but also in the tensor-valued numerator. In $2 \rightarrow 2$ scattering these integrals can involve between one and four vertices. Tensor integrals can be reduced to scalar integrals by exploiting Lorentz invariance and extending the well-known reduction procedure (Passarino and Veltman, 1979) to $n$ dimensions. The coefficients of the scalar integrals are then finite and have to be kept up to $\mathcal{O}(\varepsilon)$ or $\mathcal{O}\left(\varepsilon^{2}\right)$. All divergences are contained in the scalar integrals. They are of three different types. Ultraviolet $1 / \varepsilon$ poles appear at the upper boundary $(\infty)$ of the energy integration in the one- and two-vertex functions. They are removed, together with the universal finite terms $-\gamma_{E}+\ln (4 \pi)$, by renormalizing the bare strong coupling $\hat{g}_{s}$, wave functions, and masses $\hat{m}_{i}$ (where applicable) in the $\overline{\mathrm{MS}}$ scheme (Bardeen et al., 1978). Infrared poles arise at the lower boundary (0) of the energy integration when massless particles are exchanged between real particles, and collinear poles occur in the splitting of massless particles into two massless collinear particles. Infrared and collinear poles and double poles appear in the two-, three-, and four-vertex functions and in the derivatives of two-point functions, which play a role in external self-energy corrections. These poles are particularly cumbersome in massless QCD calculations.

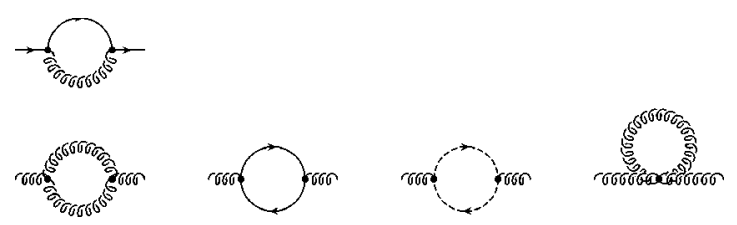

FIG. 10. Quantum chromodynamics quark (top) and gluon (bottom) self-energy diagrams. Faddeev-Popov ghosts are depicted as dashed lines.

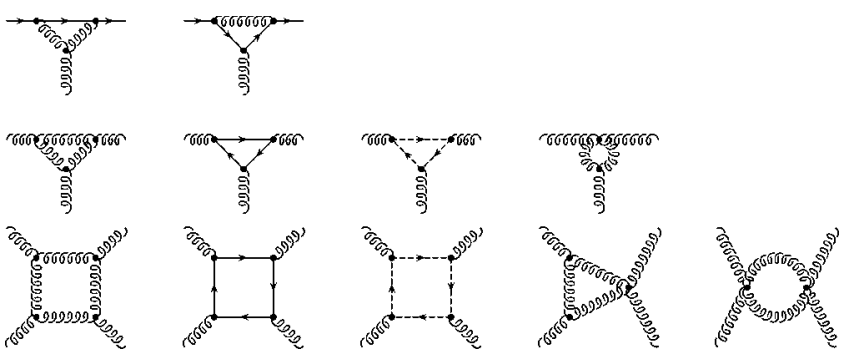

FIG. 11. Quantum chromodynamics corrections to the quarkgluon vertex (top), triple-gluon vertex (center), and four-gluon vertex (bottom).

In practice, the scalar integrals can be calculated either with Feynman parameters ('t Hooft and Veltman, 1979) or with Cutkosky cutting rules and dispersion relations ('t Hooft and Veltman, 1973). Analytical continuation of products of logarithms can lead to numerically large terms of $\pi^{2}$. The complete one-loop QCD corrections have been calculated in dimensional regularization for massless parton scattering processes involving two (Aurenche, Baier, et al., 1984a), one (Aurenche, Baier, et al., 1987), or no photons (Ellis and Sexton, 1986).

Real corrections arise in next-to-leading order by the splitting of one particle into two, as shown in Fig. 12 for massless QCD partons. These diagrams have to be attached at all possible places to the leading-order diagrams in Figs. 7-9 and lead to $2 \rightarrow 3$ processes with different kinematics. The first three diagrams could also be applied to photons, but they would constitute $\mathcal{O}(\alpha)$ corrections and should not be included at $\mathcal{O}\left(\alpha_{s}\right)$. Only the $\gamma \rightarrow q \bar{q}$ diagram plays a special role, since it provides a link at next-to-leading order between direct and resolved photoproduction (see Sec. III). Analytical expressions for the massless $2 \rightarrow 3$ scattering matrix elements have been computed in $n$ dimensions for photonphoton (Aurenche, Baier, et al., 1984a), photon-parton (Aurenche, Baier, et al., 1987), and parton-parton (Ellis and Sexton, 1986) scattering.

For single-particle production (see Secs. V and VI), it is possible to integrate the squared $2 \rightarrow 3$ matrix elements $\left|\mathcal{M}^{F, I}\right|^{2}$ analytically over the solid angle

$$
\int d \Omega=\int_{0}^{\pi} d \theta_{1} \sin ^{1-2 \varepsilon} \theta_{1} \int_{0}^{\pi} d \theta_{2} \sin ^{-2 \varepsilon} \theta_{2}
$$

of the two unresolved final-state $(F)$ or initial-state $(I)$ particles in a frame, where they have no net threemomentum. However, the integration over the invariant mass $s_{i j}=\left(p_{i}+p_{j}\right)^{2}$ of the two unobserved partons with four-momenta $p_{i}$ and $p_{j}$ has to be performed numerically (Ellis et al., 1980). These integrations are implied in the cross-section expression of Eq. (4.11). The method described above can be applied to direct (Aurenche, Fontannaz, et al., 1994a), single- (Aurenche, Fontannaz,
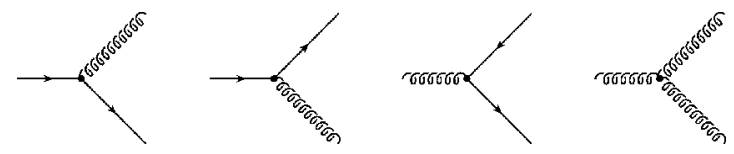

FIG. 12. Real QCD corrections through $1 \rightarrow 2$ parton splitting. 
and Guillet, 1994b), and double-resolved jet photoproduction (Aversa et al., 1989, 1990) if the phase space in Eq. (4.12) is restricted to allow for an observed jet with a small cone of angular size $\delta$ similar to the StermanWeinberg criterion. For comparisons with experimental data, finite-cone-size corrections have to be implemented by integrating the $2 \rightarrow 3$ matrix elements numerically in the finite region between the small technical cone of size $\delta$ and the larger experimental cone of size $R .{ }^{1}$ Similar methods were applied quite early in singleresolved calculations (Baer, Ohnemus, and Owens, 1989b; Bödeker, 1992a, 1992b, 1993; Gordon and Storrow, 1992b) and will be described in detail in Sec. IV.C.

If the radiated gluons in Fig. 12 become soft and/or two of the three particles become collinear, singularities appear in the propagators of the intermediate particles and have to be extracted analytically as $1 / \varepsilon$ or $1 / \varepsilon^{2}$ (double) poles. The interference of different amplitudes can furthermore involve multiple singularities, which have to be separated by partial fractioning. According to the Kinoshita-Lee-Nauenberg theorem, all soft and final-state collinear singularities from the real corrections must cancel against corresponding virtual singularities in inclusive jet cross sections. Only universal collinear singularities

$$
\left|\mathcal{M}^{I}\right|_{a b \rightarrow 123}^{2}=-\frac{1}{\varepsilon} P_{c \leftarrow a}(x)\left|\mathcal{M}^{B}\right|_{c b \rightarrow 12}^{2}+\mathcal{O}\left(\varepsilon^{0}\right)
$$

remain, which correspond to the parton splittings in Fig. 12 attached to the initial state of the Born diagrams. They are proportional to the Altarelli-Parisi splitting functions in Eq. (3.6) and are absorbed, together with scheme-dependent finite terms, into the parton densities in the proton or in the photon (see Sec. III). For the production of jets in direct, single-resolved, and doubleresolved photon-photon collisions, the soft and collinear singular parts of the NLO squared matrix elements $\left|\mathcal{M}^{V, F, I}\right|^{2}$ are collected in Tables VII, VIII, and IX. In all three cases the sums are finite, since the factorizable initial-state singularities have already been subtracted. The arguments of the logarithms $l(x)=\ln \left|x / Q^{2}\right|$ have been normalized to the arbitrary scale $Q^{2}$, which also appears in Eq. (4.11) and drops out in the total result.

The photoproduction cross section for inclusive jets,

$$
\begin{aligned}
\frac{d^{2} \sigma}{d E_{T} d \eta}= & \sum_{a, b} \int_{x_{a, \min }}^{1} d x_{a} x_{a} f_{a / A}\left(x_{a}, M_{a}^{2}\right) x_{b} f_{b / B}\left(x_{b}, M_{b}^{2}\right) \\
& \times \frac{4 E_{A} E_{T}}{2 x_{a} E_{A}-E_{T} e^{\eta}} \frac{d \sigma}{d t}
\end{aligned}
$$

\footnotetext{
${ }^{1}$ See, for example, Aversa et al., 1991; Gordon and Storrow, 1992b; Aurenche, Fontannaz, et al., 1994a; Aurenche, Fontannaz, and Guillet, 1994b; Bödeker, Kramer, and Salesch, 1994; Greco and Vicini, 1994; Kramer and Salesch, 1994a; Klasen, Kramer, and Salesch, 1995. The double-resolved contributions in these calculations were all based on the same results by Aversa et al. $(1989,1990)$. With the exception of the first two calculations, they were found to agree numerically with later calculations using different methods (Klasen, 1996b; Aurenche, Bourhis, et al., 2000).
}

TABLE VII. Cancellation of soft and collinear singularities from virtual and final-state next-to-leading-order corrections for direct photon scattering.

\begin{tabular}{lccc}
\hline \hline Process & $\begin{array}{c}\text { Color } \\
\text { factor }\end{array}$ & Correction & $\begin{array}{l}\text { Singular parts of } \\
\left|\mathcal{M}^{V, F, I}\right|^{2} /\left|\mathcal{M}^{B}\right|^{2}\end{array}$ \\
\hline$\gamma \gamma \rightarrow q \bar{q}$ & $C_{F}$ & Virtual & {$\left[-\frac{2}{\varepsilon^{2}}-\frac{1}{\varepsilon}[3-2 l(t)]\right]$} \\
& & Final & {$\left[+\frac{2}{\varepsilon^{2}}+\frac{1}{\varepsilon}[3-2 l(t)]\right]$} \\
\hline
\end{tabular}

depends on the transverse energy $E_{T}$ and pseudorapidity $\eta$ of the observed jet. In Eq. (4.14), $\eta$ is defined in the laboratory frame with particle $A$ traveling in the positive $z$ direction. It is related to the pseudorapidity in the hadronic center-of-mass frame $\eta^{*}=\eta+1 / 2 \ln \left(E_{B} / E_{A}\right)$ by a simple boost. From the final-state variables $E_{T}$ and $\eta$ and from the energies $E_{A}$ and $E_{B}$ of the initial leptons or hadrons, the four-momenta of the incoming partons $p_{a, b}=E_{A, B}\left(x_{a, b}, 0,0, \pm x_{a, b}\right)$ cannot be fully reconstructed. Instead the partonic cross section $d \sigma / d t$ is integrated over $x_{a}$ with

$$
\begin{aligned}
& x_{a, \min }=\frac{E_{B} E_{T} e^{\eta}}{2 E_{A} E_{B}-E_{A} E_{T} e^{-\eta}}, \\
& x_{b}=\frac{x_{a} E_{A} E_{T} e^{-\eta}}{2 x_{a} E_{A} E_{B}-E_{B} E_{T} e^{\eta}},
\end{aligned}
$$

and convolved with the parton densities $f_{a / A}\left(x_{a}, M_{a}^{2}\right)$ and $f_{b / B}\left(x_{b}, M_{b}^{2}\right)$ at the factorization scales $M_{a, b}$. The density of partons $i$ in initial leptons $l$ is given by the convolution

\begin{tabular}{|c|c|c|c|}
\hline Process & $\begin{array}{l}\text { Color } \\
\text { factor }\end{array}$ & Correction & $\begin{array}{l}\text { Singular parts of } \\
\left|\mathcal{M}^{V, F, I}\right|^{2} /\left|\mathcal{M}^{B}\right|^{2}\end{array}$ \\
\hline \multirow[t]{9}{*}{$\gamma q \rightarrow g q$} & $C_{F}$ & Virtual & {$\left[-\frac{2}{\varepsilon^{2}}-\frac{1}{\varepsilon}[3-2 l(t)]\right]$} \\
\hline & & Final & {$\left[+\frac{1}{\varepsilon^{2}}+\frac{1}{2 \varepsilon}[3-2 l(t)]\right]$} \\
\hline & & Initial & {$\left[+\frac{1}{\varepsilon^{2}}+\frac{1}{2 \varepsilon}[3-2 l(t)]\right]$} \\
\hline & $N_{C}$ & Virtual & {$\left[-\frac{1}{\varepsilon^{2}}-\frac{1}{2 \varepsilon}\left[\frac{11}{3}-2 l(s / t)-2 l(u)\right]\right.$} \\
\hline & & Final & {$\left[+\frac{1}{\varepsilon^{2}}+\frac{1}{2 \varepsilon}\left[\frac{11}{3}-l(s / t)-l(u)\right]\right]$} \\
\hline & & Initial & {$\left[+\frac{1}{2 \varepsilon}[-l(s / t)-l(u)]\right]$} \\
\hline & $N_{f}$ & Virtual & $+\frac{1}{3 \varepsilon}$ \\
\hline & & & 1 \\
\hline & & Final & $-\overline{3 \varepsilon}$ \\
\hline
\end{tabular}

TABLE VIII. Cancellation of soft and collinear singularities from virtual, final-state, and initial-state next-to-leading-order corrections for single-resolved photon scattering and different color factors. 
TABLE IX. Cancellation of soft and collinear singularities from virtual, final-state, and initial-state NLO corrections for double-resolved photon scattering and different color factors.

\begin{tabular}{|c|c|c|c|}
\hline Process & $\begin{array}{l}\text { Color } \\
\text { factor }\end{array}$ & Correction & $\begin{array}{l}\text { Singular parts of } \\
\left|\mathcal{M}^{V, F, I}\right|^{2} /\left|\mathcal{M}^{B}\right|^{2}\end{array}$ \\
\hline \multirow[t]{6}{*}{$q q^{\prime} \rightarrow q q^{\prime}$} & $C_{F}$ & Virtual & {$\left[-\frac{4}{\varepsilon^{2}}-\frac{1}{\varepsilon}[6+8 l(s / u)-4 l(t)]\right]$} \\
\hline & & Final & {$\left[+\frac{2}{\varepsilon^{2}}+\frac{1}{\varepsilon}[3+4 l(s / u)-2 l(t)]\right]$} \\
\hline & & Initial & {$\left[+\frac{2}{\varepsilon^{2}}+\frac{1}{\varepsilon}[3+4 l(s / u)-2 l(t)]\right]$} \\
\hline & $N_{C}$ & Virtual & {$\left[+\frac{1}{\varepsilon}[4 l(s)-2 l(u)-2 l(t)]\right]$} \\
\hline & & Final & {$\left[-\frac{1}{\varepsilon}[2 l(s)-l(u)-l(t)]\right]$} \\
\hline & & Initial & {$\left[-\frac{1}{\varepsilon}[2 l(s)-l(u)-l(t)]\right]$} \\
\hline \multirow[t]{6}{*}{$q q \rightarrow q q$} & $C_{F}$ & Virtual & {$\left[-\frac{4}{\varepsilon^{2}}-\frac{1}{\varepsilon}[6+4 l(s / t)-4 l(u)]\right]$} \\
\hline & & Final & {$\left[+\frac{2}{\varepsilon^{2}}+\frac{1}{\varepsilon}[3+2 l(s / t)-2 l(u)]\right]$} \\
\hline & & Initial & {$\left[+\frac{2}{\varepsilon^{2}}+\frac{1}{\varepsilon}[3+2 l(s / t)-2 l(u)]\right]$} \\
\hline & $N_{C}$ & Virtual & {$\left[+\frac{2}{\varepsilon}[2 l(s)-l(t)-l(u)]\right]$} \\
\hline & & Final & {$\left[-\frac{1}{\varepsilon}[2 l(s)-l(t)-l(u)]\right]$} \\
\hline & & Initial & {$\left[-\frac{1}{\varepsilon}[2 l(s)-l(t)-l(u)]\right]$} \\
\hline \multirow[t]{9}{*}{$q \bar{q} \rightarrow g g$} & $C_{F}$ & Virtual & {$\left[-\frac{2}{\varepsilon^{2}}-\frac{3}{\varepsilon}\right]$} \\
\hline & & Initial & {$\left[+\frac{2}{\varepsilon^{2}}+\frac{3}{\varepsilon}\right]$} \\
\hline & $N_{C}$ & Virtual & {$\left[-\frac{2}{\varepsilon^{2}}-\frac{11}{3 \varepsilon}\right]$} \\
\hline & & Final & {$\left[+\frac{2}{\varepsilon^{2}}+\frac{11}{3 \varepsilon}\right]$} \\
\hline & $N_{f}$ & Virtual & $+\frac{2}{3 \varepsilon}$ \\
\hline & & Final & $-\frac{1}{3 \varepsilon}$ \\
\hline & & Initial & $-\frac{1}{3 \varepsilon}$ \\
\hline & 1 & Virtual & $+\frac{1}{\varepsilon} l(s)\left[\left(4 N_{C}^{3} C_{F}+\frac{4 C_{F}}{N_{C}}\right) \frac{t^{2}+u^{2}}{t u}-16 N_{C}^{2} C_{F}^{2} \frac{t^{2}+u^{2}}{s^{2}}\right] /\left|\mathcal{M}^{B}\right|^{2}$ \\
\hline & & Final & $-\frac{1}{2 \varepsilon} l(s)\left[\left(4 N_{C}^{3} C_{F}+\frac{4 C_{F}}{N_{C}}\right) \frac{t^{2}+u^{2}}{t u}-16 N_{C}^{2} C_{F}^{2} \frac{t^{2}+u^{2}}{s^{2}}\right] /\left|\mathcal{M}^{B}\right|^{2}$ \\
\hline
\end{tabular}


TABLE IX. (Continued).

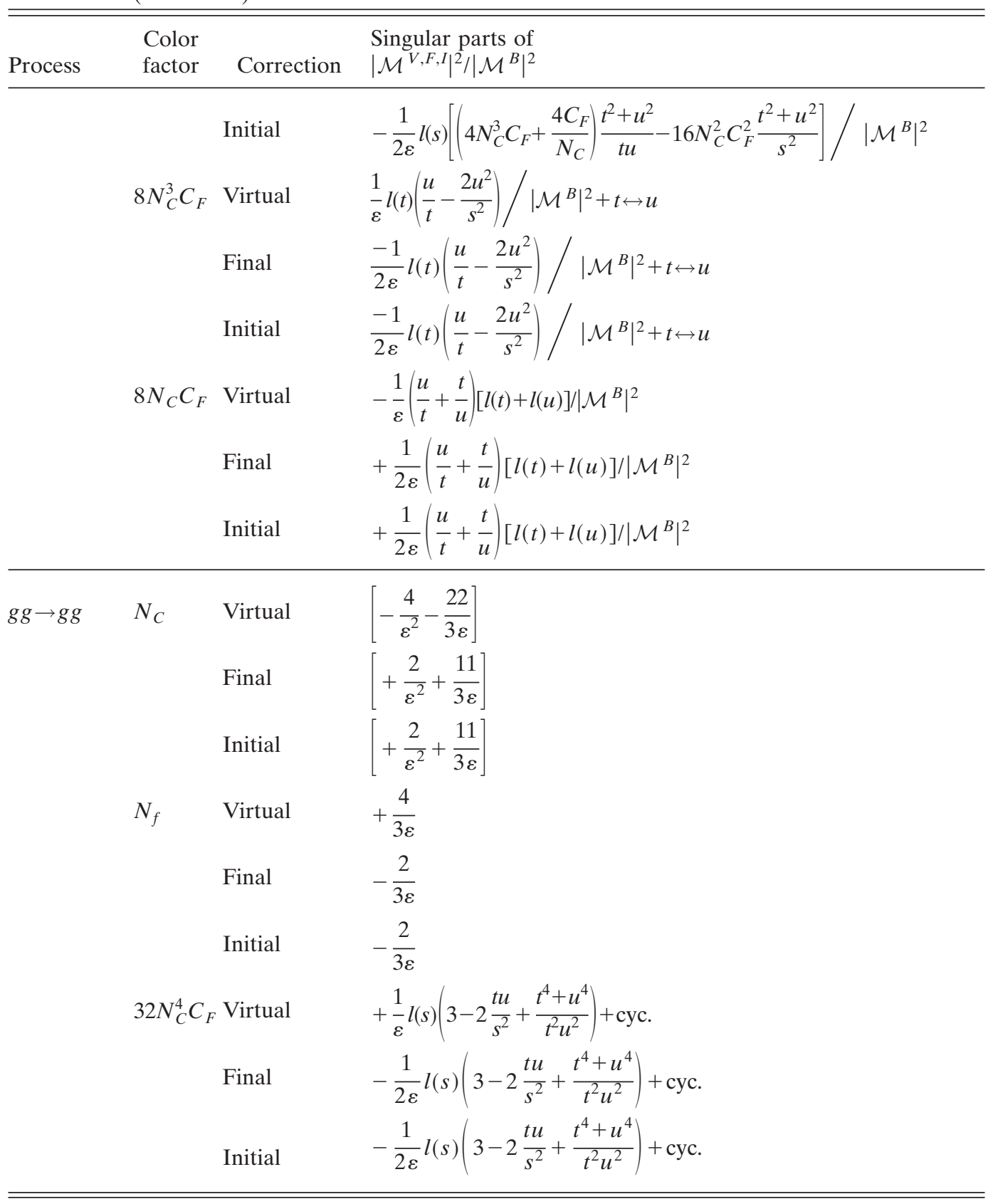

$$
f_{i / l}\left(x, M^{2}\right)=\left[f_{i / \gamma}\left(M^{2}\right) \otimes f_{\gamma / l}\right](x)
$$

of the photon spectrum $f_{\gamma / l}(x)$ as described in Sec. II with the parton densities in the photon $f_{i / \gamma}\left(x, M^{2}\right)$ (see Sec. III), so that the two cannot be uniquely disentangled.

Due to the truncation of the perturbative series, fixedorder predictions depend on the scales at which the strong coupling $\alpha_{s}(\mu)$ is renormalized and at which the collinear initial-state singularities are factorized into the parton densities $f_{i / a}\left(x, M^{2}\right)$. In leading order, the logarithmic scale dependence is generally quite strong. It is reduced in next-to-leading order through the explicit logarithmic dependences in the virtual and real initialstate corrections,

$$
\begin{aligned}
& \left|\mathcal{M}^{V}\right|_{a b \rightarrow 12}^{2}=\ln \left(\frac{\mu^{2}}{Q^{2}}\right)\left|\mathcal{M}^{B}\right|_{a b \rightarrow 12}^{2} \beta_{0}+\cdots, \\
& \left|\mathcal{M}^{I}\right|_{a b \rightarrow 123}^{2}=\ln \left(\frac{M^{2}}{Q^{2}}\right)\left|\mathcal{M}^{B}\right|_{c b \rightarrow 12}^{2} P_{c \leftarrow a}(x)+\cdots .
\end{aligned}
$$

For the renormalization scale $\mu$, this is demonstrated in Fig. 13, where the total single-jet photoproduction cross section Eq. (4.14) has been calculated using HERA kinematics $\left(E_{p}=820 \mathrm{GeV}\right.$ and $\left.E_{e}=26.7 \mathrm{GeV}\right)$. Unless stated otherwise, NLO parton densities in the $\overline{\mathrm{MS}}$ scheme for the proton (Lai et al., 1995) and photon (Glück, Reya, and Vogt, 1992b) have been used with 


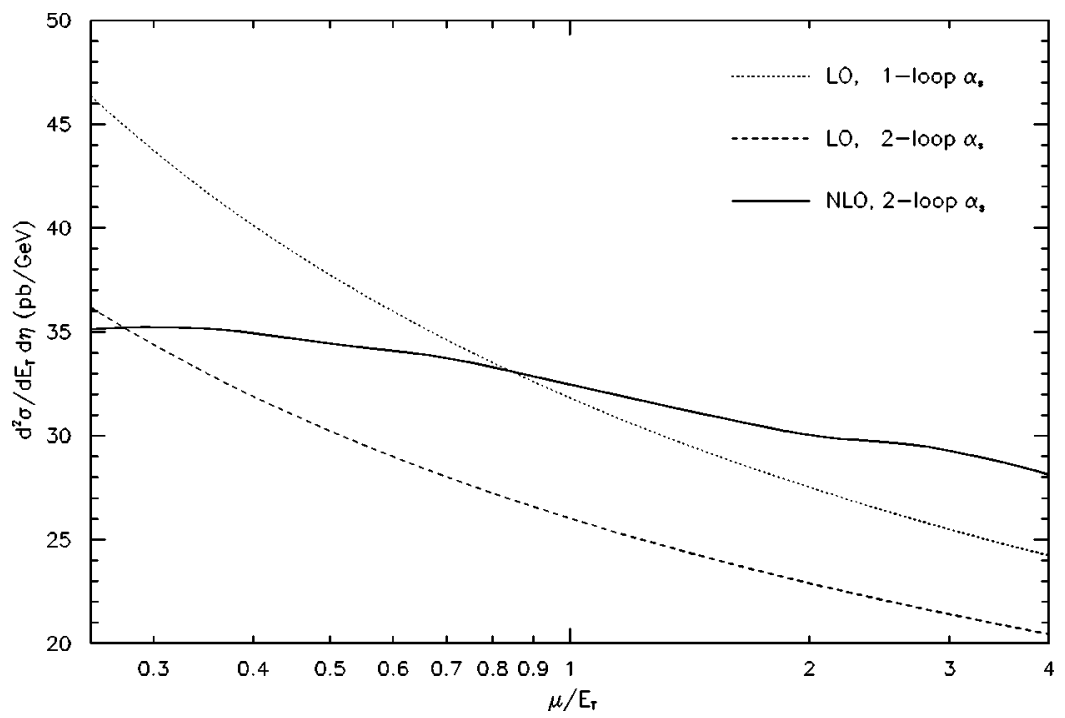

FIG. 13. Renormalization scale dependence of the single-jet cross section $d^{2} \sigma / D E_{T} d \eta$ at HERA with $E_{T}=20 \mathrm{GeV}$ and $\eta=1$ in leading order (LO; dotted and dashed curves) and next-to-leading order (NLO; solid curve).

$\Lambda_{\overline{\mathrm{MS}}}^{N_{f}=4}=239 \mathrm{MeV}$. For a particular choice of $\mu$, the NLO corrections vanish and the perturbative series apparently converges very fast (Grunberg, 1980). If a oneloop $\alpha_{s}$ formula is used in the leading-order calculation, this happens at $\mu \simeq M_{p}=M_{\gamma}=E_{T}$, while for two-loop $\alpha_{s}$ it happens at $\mu \simeq E_{T} / 4$. At this scale, the NLO curve also exhibits a local maximum and has minimal sensitivity to scale variations (Stevenson, 1981a, 1981b). However, the dependences on $M_{p}$ and $M_{\gamma}$ also have to be considered and can spoil this simple picture. If all scales are varied simultaneously, the principles of fastest convergence and minimal sensitivity lead indeed to unreasonably low scale choices (Klasen, 1996b). The situation becomes even more complicated if more than one scale is involved, as is the case in heavy-quark or deep-inelasticscattering (DIS) jet production.

In photoproduction, the dependence on the photon factorization scale $M_{\gamma}$ is of particular interest, since this scale defines the separation of direct and resolved processes. While the direct leading-order cross section is manifestly independent of $M_{\gamma}$, the resolved leadingorder cross section depends logarithmically on $M_{\gamma}$ through the leading-order parton densities in the photon. This dependence is canceled almost exactly by the explicit logarithmic dependence of the NLO direct term, as can be seen in Fig. 14 (Bödeker, Kramer, and Salesch, 1994; Klasen, 1996b; Klasen, Kleinwort, and Kramer, 1998). However, the resolved cross section rises faster in next-to-leading order than in leading order (Klasen, 1996b). This reintroduces a (weaker) dependence in the total NLO result, which would eventually be canceled by the direct NNLO contribution.

The HERA experiments H1 and ZEUS select photoproduction events by tagging and antitagging the scattered electrons, respectively, which limits the maximal squared virtuality of the exchanged photon to $Q^{2}$

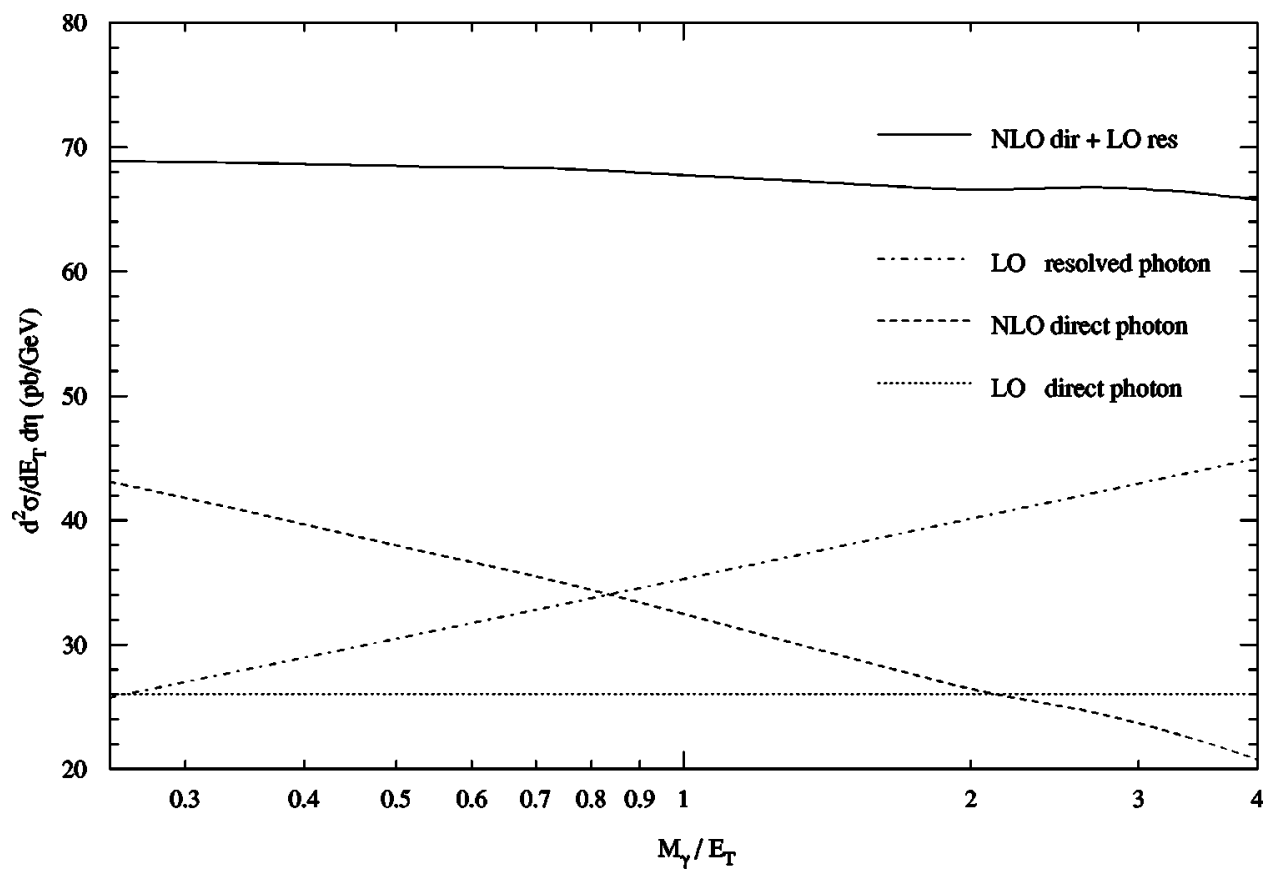

FIG. 14. Photon factorization scale dependence of the singlejet cross section $d^{2} \sigma / d E_{T} d \eta$ at HERA with $E_{T}=20 \mathrm{GeV}$ and $\eta=1$. The leading-order direct curve (dotted) is independent of $M_{\gamma}$, as is the sum (solid curve) of NLO direct (dashed) and leading-order resolved (dot-dashed) contributions. 


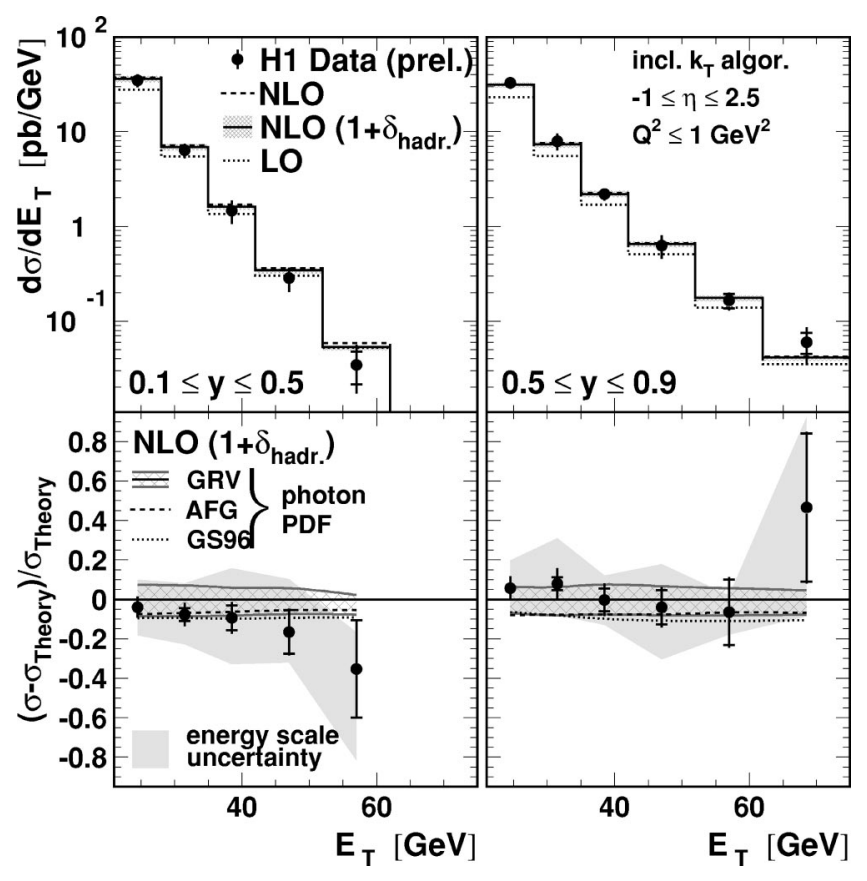

FIG. 15. Transverse energy dependence of the total single-jet photoproduction cross section compared to preliminary H1 data. From Adloff et al., 2001a.

$<0.01-1$ and $1-4 \mathrm{GeV}^{2}$. The energy fraction $y$ transferred to the photon is determined from the scattered electron energy $E_{e}^{\prime}$ with $y=1-E_{e}^{\prime} / E_{e}$ or with the Jacquet-Blondel method,

$$
y=\frac{1}{2 E_{e}} \sum_{i}\left(E_{i}-p_{z_{i}}\right),
$$

from the energies $E_{i}$ and longitudinal momenta $p_{z_{i}}$ of the final hadrons (Amaldi et al., 1979). Single-jet production has been analyzed with the cone algorithm and $R=1$ by H1 (Abt et al., 1993; Aid et al., 1996a) and ZEUS (Derrick et al., 1995a; Breitweg et al., 1998a) and has been compared to various NLO calculations (Aurenche, Fontannaz, and Guillet, 1994b; Klasen, Kramer, and Salesch, 1995; Klasen, 1996a, 1996b, 1997a; Harris and Owens, 1997, 1998; Klasen, Kleinwort, and Kramer, 1998). In these early comparisons at rather low transverse energies of $\sim 10 \mathrm{GeV}$, the theory overestimated the data in the backward region, since no hadronization corrections had been applied, while it underestimated the data in the forward region, triggering speculations about additional soft interactions between the hard jets and the proton remnant. In more recent analyses (Breitweg et al., 1998b; Adloff et al., 2001a), the invariant $k_{T}$ algorithm has been used. The measured $\mathrm{H} 1$ cross section in Fig. 15 now extends out to $E_{T}<75 \mathrm{GeV}$ for large $y$. It falls by three orders of magnitude and agrees remarkably well with the NLO prediction (Frixione and Ridolfi, 1997). The hadronization corrections $\delta_{\text {hadr. }}$ are much smaller than the NLO corrections, and the remaining theoretical uncertainty from varying the scale $\mu=M_{p}$ $=M_{\gamma}=E_{T} / 2$ by a factor of 2 up and down is modest. Different photon densities give slightly different normal-

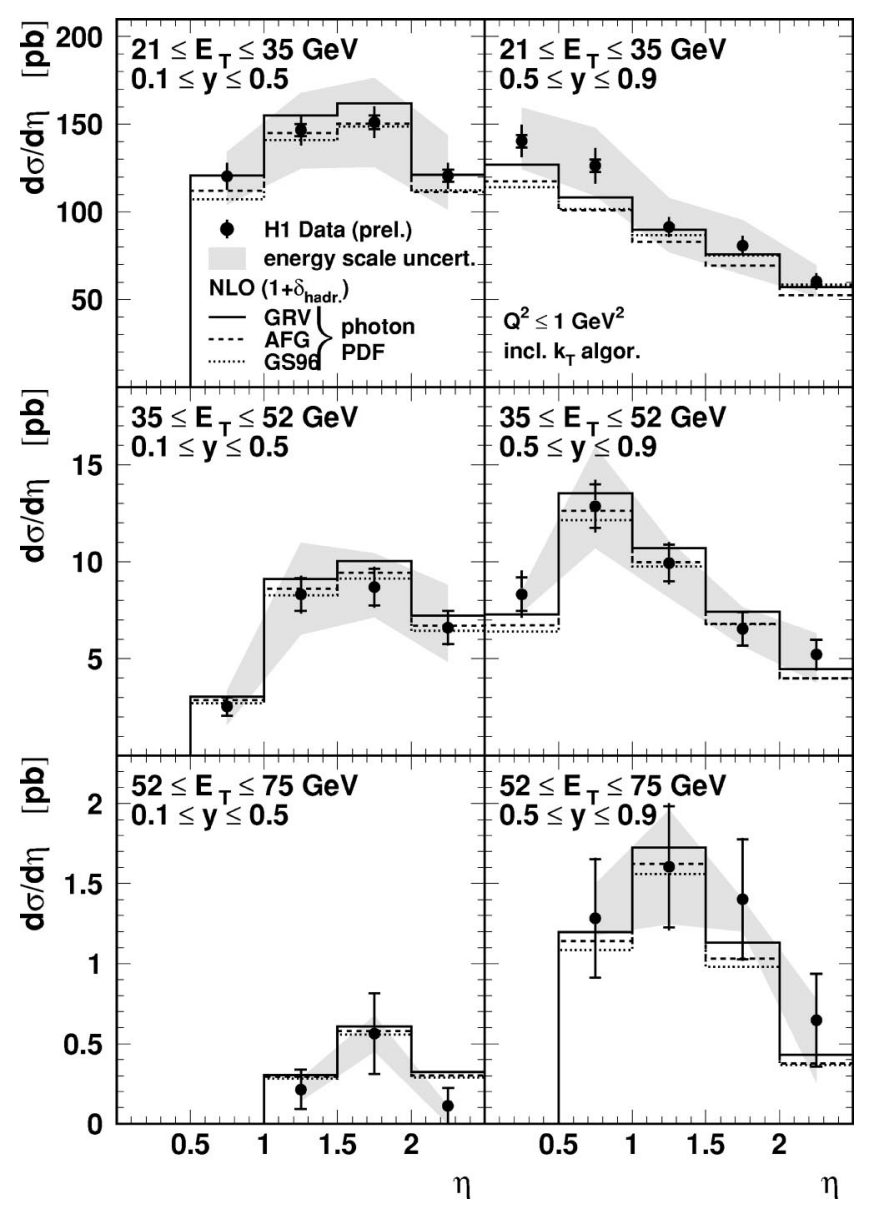

FIG. 16. Pseudorapidity dependence of the total single-jet photoproduction cross section compared to preliminary $\mathrm{H} 1$ data. From Adloff et al., 2001a.

izations, but the experimental uncertainty is still too large to draw definite conclusions. Single-jet pseudorapidity distributions are shown in Fig. 16. The maximum of the cross section is shifted in the electron direction (low $\eta$ ) when increasing $y$ and lowering $E_{T}$. The H1 data are in general well described by the NLO prediction, perhaps best with Glück-Reya-Vogt photon densities, but the experimental and theoretical (not shown) uncertainties are still quite large. Direct photoproduction dominates at large $E_{T}$ and small $\eta$, while resolved processes are more important at smaller $E_{T}$ and larger $\eta$.

A good measure for the width of a produced jet is the jet shape

$$
\rho(r \leqslant R)=1-\frac{\int d E_{T} d \eta E_{T} d^{2} \sigma^{\mathrm{NLO} /\left(d E_{T} d \eta\right)}}{\int d E_{T} d \eta E_{T} d^{2} \sigma^{\mathrm{LO}} /\left(d E_{T} d \eta\right)},
$$

which is the average fraction of a jet's transverse energy that lies inside a concentric inner cone with radius $r$ $\leqslant R$. It has been calculated in resolved (Kramer and Salesch, 1993, 1994b; Klasen, 1997a; Klasen and Kramer, 1997b) and direct (Klasen, 1997a; Klasen and Kramer, 

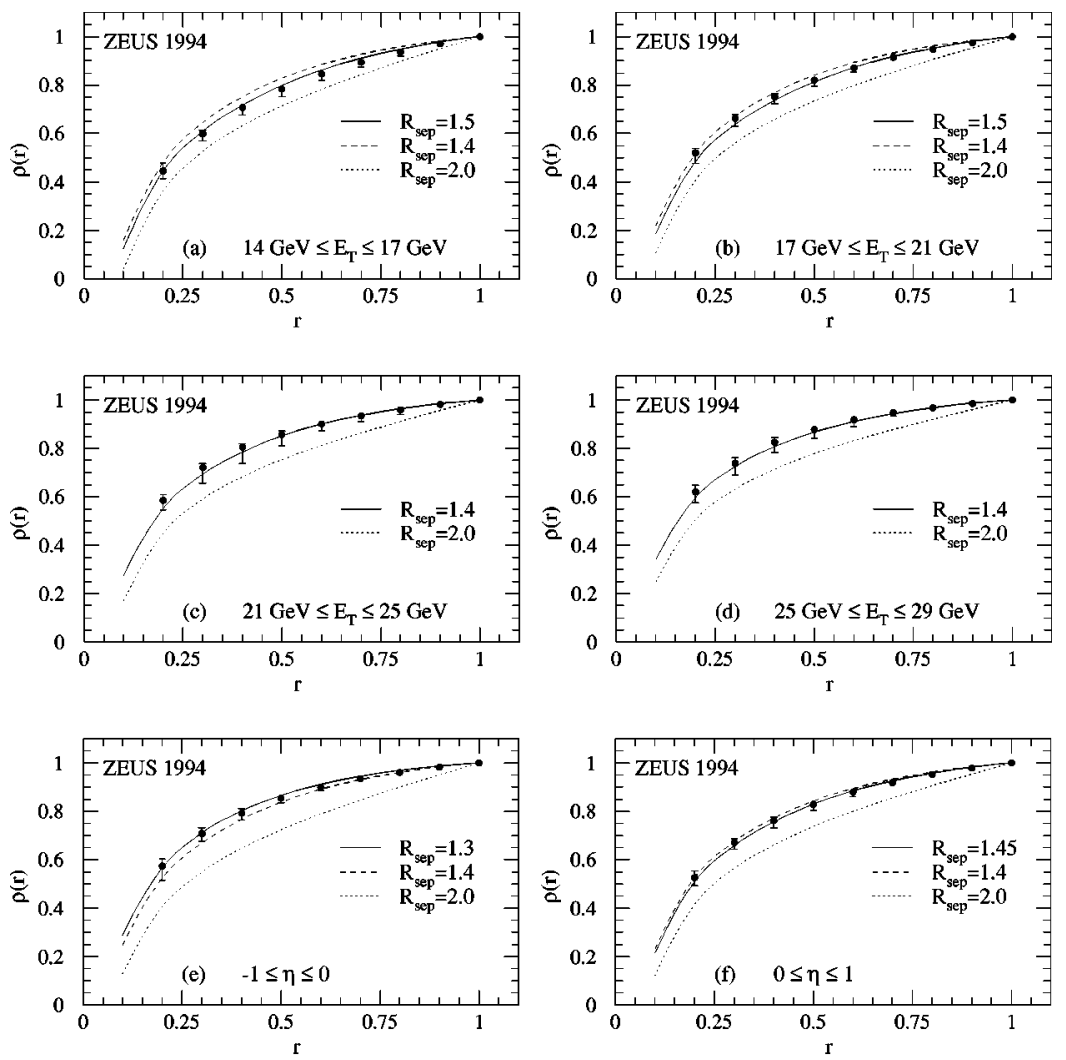

FIG. 17. Jet shapes $\rho(r)$ for single-jet photoproduction: (a)-(d) integrated over $-1<\eta$ $<2$ and four different regions of $E_{T} ;(\mathrm{e})-(\mathrm{h})$ integrated over $E_{T}>14 \mathrm{GeV}$ and four different regions of $\eta$. 1994 data from ZEUS (Breitweg et al., 1998d) are compared to NLO results using the cone algorithm with $R=1$ and different values of $R_{\text {sep }}$.
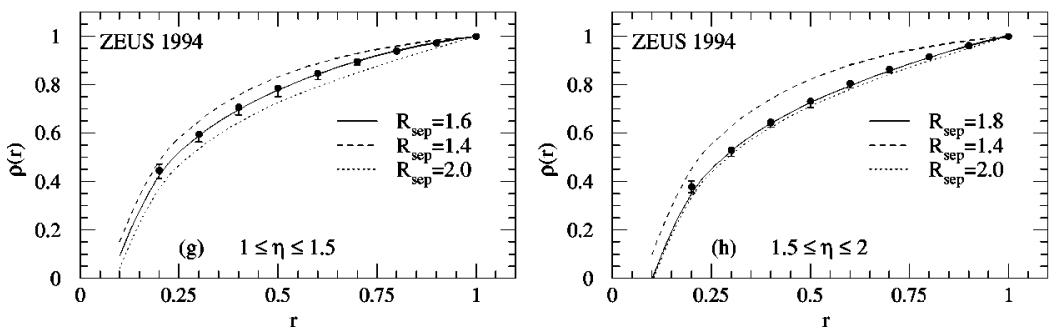

$1997 b)$ photoproduction by integrating the $E_{T}$-weighted NLO $2 \rightarrow 3$ cross section in the nonsingular region between $r$ and $R$. In Fig. 17 jet shapes for single-jet photoproduction are compared to ZEUS data (Breitweg et al., 1998d) for different transverse energy and pseudorapidity intervals. It is obvious that the data and the NLO predictions rise more steeply for large values of $E_{T}$ and small values of $\eta$, where the jets become narrower. Using leading-order Monte Carlo predictions, the ZEUS Collaboration has recently been able to associate thick and thin jets with gluon and quark jets (Breitweg et al., 2000a).

Inclusive jet production in photon-photon scattering has been measured at the $e^{+} e^{-}$collider TRISTAN $(\sqrt{S}=58 \mathrm{GeV})$ by the TOPAZ (Hayashii et al., 1993) and AMY (Kim et al., 1994) Collaborations, where scattered electrons were antitagged at small angles ( $\theta_{\max }$ $=227$ and $56 \mathrm{mrad}$ ). The OPAL Collaboration (Ackerstaff et al., 1997) performed a measurement at LEP1.5 $(\sqrt{S}=133 \mathrm{GeV})$ with a maximal electron scattering angle of $33 \mathrm{mrad}$. All experiments used the cone algorithm and $R=1$. As shown in Fig. 18, the AMY data are well described by the sum of NLO direct, single-, and double-resolved processes (Kleinwort and Kramer, 1996a) with Glück-Reya-Vogt photon densities. The same observation is made in NLO comparisons with the TOPAZ (Aurenche, Fontannaz, et al., 1994a; Kleinwort and Kramer, 1996a) and OPAL (Klasen, Kleinwort, and Kramer, 1998) data. At comparable values of $x_{T}$ $=2 E_{T} / \sqrt{S}$, the jet width measured in photon-photon collisions is approximately the same as in photon-hadron collisions (Adachi et al., 1999).

\section{Dijets}

Next-to-leading-order calculations for dijet photoproduction differ significantly from those for single jets with respect to the real corrections. While the phase space of the two unobserved partons could be integrated analytically for single jets [see Eq. (4.12)], it is now restricted by the definition of the second observed jet. The third unobserved parton momentum $p_{3}$ still has to be integrated out.

In the phase-space slicing method, this is done analytically in the soft and collinear regions, which can be defined by limiting the invariant masses of two unresolved partons $s_{i 3}=\left(p_{i}+p_{3}\right)^{2}<y s$ (Gutbrod, Kramer, and Schi- 


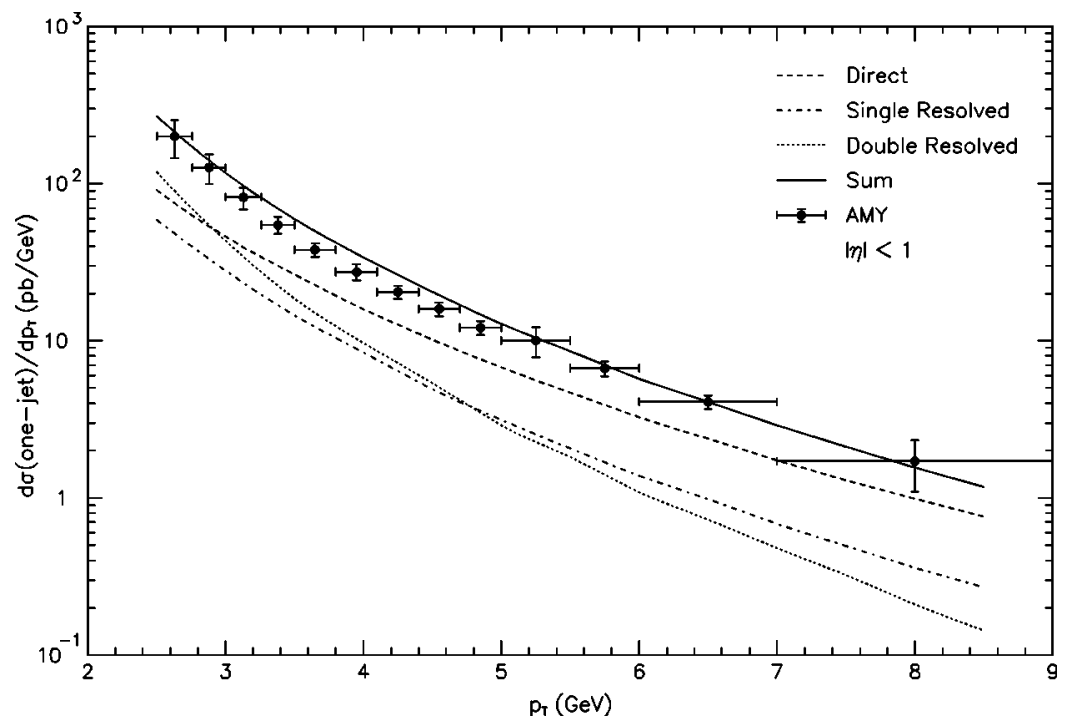

FIG. 18. Transverse momentum distribution of single jets produced in photon-photon collisions compared to AMY data. From Kleinwort and Kramer, 1996a.

erholz, 1984; Kramer and Lampe, 1989) or by applying separate soft and collinear cutoffs on $E_{3}<\epsilon \sqrt{s} / 2$ and $\theta_{i 3}<\delta$ (Fabricius et al., 1981; Gutbrod, Kramer, and Schierholz, 1984), on $E_{3}<\delta_{s} \sqrt{s} / 2$ and $s_{i 3}<\delta_{c} s$ (Baer, Ohnemus, and Owens, 1989b; Harris and Owens, 2001), or on $E_{T_{3}}<p_{T m}$ and $R_{i 3}<R_{c}$ (Aurenche, Bourhis, et al., 2000). Outside the singular regions, the real corrections are integrated numerically, so that the cross sections become independent of the technical cutoffs and an experimental jet definition can be implemented. While the $y$-cut method has been applied to direct (Kleinwort and Kramer, 1996a, 1996b, 1997; Klasen, Kleinwort, and Kramer, 1998), single-resolved (Klasen and Kramer, 1996a, 1996b, 1997a), and double-resolved (Klasen and Kramer, 1997a) dijet photoproduction, the $\delta_{s, c}$ method (Baer, Ohnemus, and Owens, 1989b; Harris and Owens, 1997, 1998) and $p_{T m}, R_{c}$ method (Aurenche, Bourhis, et al., 2000) have been applied only to the single- and double-resolved cases. All these results were found to be in good agreement with each other (Harris, Klasen, and Vossebeld, 1999; Aurenche, Bourhis, et al. 2000).

In the subtraction method, $p_{3}$ is integrated numerically in the soft and collinear regions. The $2 \rightarrow 3$ matrix elements then have to be regulated by subtracting them in a finite phase-space volume, setting $p_{3}=0$ everywhere except in the singular propagator denominators. The subtracted terms are then integrated analytically in the same phase-space volume and added to the virtual corrections to allow for an analytical cancellation of the soft and collinear poles (Ellis, Ross, and Terrano, 1981). Several solutions have been proposed to obtain numerical stability with this method (Ellis, Kunszt, and Soper, 1989a, 1989b, 1990; Kunszt and Soper, 1992). Quantum chromodynamics dipole factorization formulas allow for a straightforward computation of the soft and collinear subtraction terms (Catani and Seymour, 1996, 1997). The subtraction method has been applied to singleresolved (Bödeker, 1992a, 1992b, 1993; Frixione and Ridolfi, 1997) and double-resolved (Frixione and Ridolfi, 1997) dijet photoproduction.
In addition to the transverse energy $E_{T}$ and pseudorapidity $\eta_{1}$ of the first jet, the inclusive dijet cross section

$\frac{d^{3} \sigma}{d E_{T}^{2} d \eta_{1} d \eta_{2}}=\sum_{a, b} x_{a} f_{a / A}\left(x_{a}, M_{a}^{2}\right) x_{b} f_{b / B}\left(x_{b}, M_{b}^{2}\right) \frac{d \sigma}{d t}$

depends on the pseudorapidity of the second jet $\eta_{2}$. In leading order only two jets with equal transverse energies can be produced, and the observed momentum fractions of the partons in the initial electrons or hadrons,

$$
x_{a, b}^{\mathrm{obs}}=\sum_{i=1}^{2} E_{T_{i}} e^{ \pm \eta_{i} /\left(2 E_{A, B}\right),}
$$

equal the true momentum fractions $x_{a, b}$. If the energy transfer $y=E_{\gamma} / E_{e}$ is known [see Eq. (4.19)], momentum fractions for the partons in photons $x_{\gamma}^{\text {obs }}=x_{a, b}^{\text {obs }} / y$ can be deduced. In next-to-leading order, where a third jet can be present, the observed momentum fractions are defined by the sums over the two jets with highest $E_{T}$, and they match the true momentum fractions only approximately. Furthermore, the transverse energies of the two hardest jets need no longer be equal to each other. Even worse, for equal $E_{T}$ cuts and maximal azimuthal distance $\Delta \phi=\phi_{1}-\phi_{2}=\pi$ the NLO prediction becomes sensitive to the method chosen for the integration of soft and collinear singularities, strongly scale dependent, and thus unreliable (Klasen and Kramer, 1996a; Frixione and Ridolfi, 1997; Harris and Owens, 1997). This sensitivity also propagates into the region of large observed momentum fractions (Aurenche, Bourhis, et al., 2000). Two proposed solutions are to allow for a small difference in the theoretical $E_{T}$ cuts or for a small technical cutoff dependence, but it is preferable to cut instead on the average $\bar{E}_{T}=\left(E_{T_{1}}+E_{T_{2}}\right) / 2$. Similarly one can define $\Delta E_{T}=E_{T_{1}}-E_{T_{2}}, \quad \bar{\eta}=\left(\eta_{1}+\eta_{2}\right) / 2, \quad$ and $\quad \Delta \eta=\eta_{1}-\eta_{2}$, which is related to the cosine of the center-of-mass scattering angle $\cos \theta^{*}=\tanh (\Delta \eta / 2)$. The dijet cross section then takes the form 

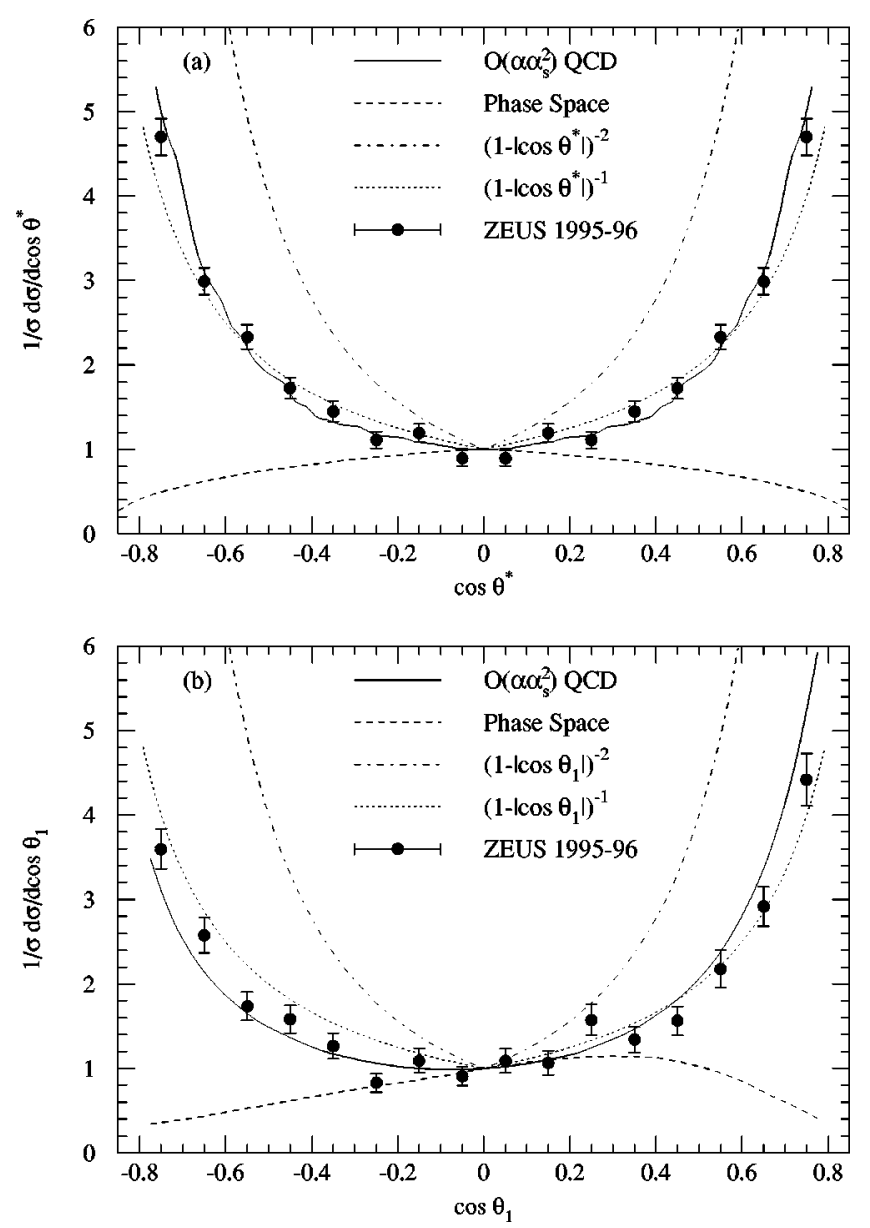

FIG. 19. Dependences of the (a) dijet and (b) three-jet cross sections on the fastest jet scattering angle, normalized at $\cos \theta=0$. The ZEUS dijet (Derrick et al., 1997a) and three-jet (Breitweg et al., 1998e) data are well described by the QCD predictions, but not by the pure phase-space distributions, and they favor the single $1 / t$ pole for massless fermion exchange over the double $1 / t^{2}$ pole for massless boson exchange.

$$
\frac{d^{3} \sigma}{d x_{a} d x_{b} d \cos \theta^{*}}=\frac{2}{s} \frac{d^{3} \sigma}{d E_{T}^{2} d \eta_{1} d \eta_{2}},
$$

which is particularly useful to determine the parton densities and scattering processes.

The $\cos \theta^{*}$ dependence at HERA is shown in Fig. 19(a) for dijet masses $M_{12}=\sqrt{\left(p_{1}+p_{2}\right)^{2}}>47 \mathrm{GeV}$ and the $k_{T}$ algorithm. The ZEUS data (Derrick et al., 1997a) show good agreement with the QCD prediction (solid curve), but clearly disagree with the pure phase-space distribution (dashed curve) and the Rutherford scattering form at small angle $\left(1-\left|\cos \theta^{*}\right|\right)^{-2}$, which is characteristic for massless vector-boson exchange in the resolved processes. On the other hand, the data agree very well with the less singular form $\left(1-\left|\cos \theta^{*}\right|\right)^{-1}$, which is typical for massless fermion exchange in the direct processes, indicating that the direct processes dominate over resolved processes in this kinematic region. A similar behavior is observed in three-jet cross sections with $M_{123}=\sqrt{\left(p_{1}+p_{2}+p_{3}\right)^{2}}>50 \mathrm{GeV}$ for the scattering angle of the leading jet [see Fig. 19(b); Breitweg et al., 1998e;

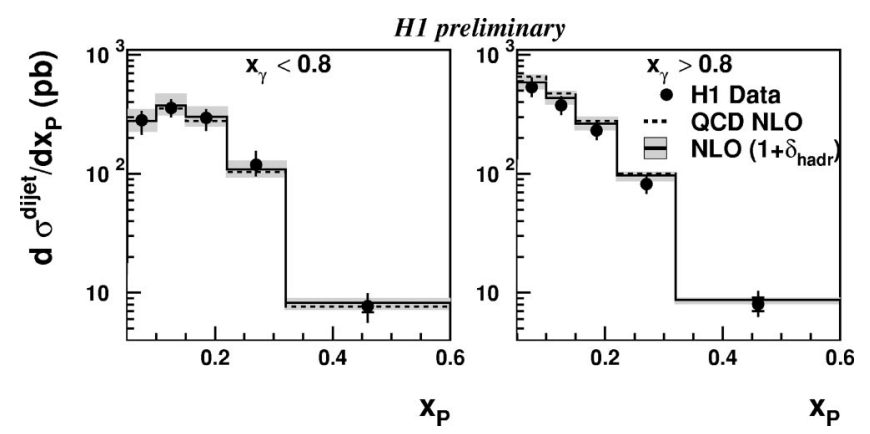

FIG. 20. Dependence of the dijet photoproduction cross section on the observed parton momentum fraction in the proton compared to preliminary H1 data (from Adloff et al., 2001b). For "resolved" photons (left), the distribution is quarklike, while for "direct" photons (right) it is gluonlike, as is to be expected from the contributing parton scattering diagrams.

Klasen, 1999a]. Next-to-leading-order QCD also describes earlier ZEUS data using the cone algorithm at lower values of $M_{12}\left(M_{12}>23 \mathrm{GeV}\right.$; Derrick et al., 1996), which have been divided into a flatter "direct" $\left(x_{\gamma}^{\mathrm{obs}}>0.75\right)$ and a steeper "resolved" $\left(x_{\gamma}^{\mathrm{obs}}<0.75\right)$ sample (Harris and Owens, 1997).

Similar analyses have recently been carried out with the $k_{T}$ algorithm by H1 (Adloff et al., 2001b) and ZEUS (Breitweg et al., 2000b), which also measured distributions of the observed parton momentum fractions in the proton (only H1) and photon with asymmetric cuts on $E_{T_{1}}>25 \mathrm{GeV}$ and $E_{T_{2}}>15 \mathrm{GeV}$ (14 and $11 \mathrm{GeV}$ in the ZEUS case). The H1 $x_{p}^{\text {obs }}$ distribution (Fig. 20) is well described by the CTEQ5M (Lai et al., 2000), proton densities but it is not sensitive to the poorly constrained gluon density at very small or large $x_{p}$. The $\mathrm{H} 1 x_{\gamma}^{\text {obs }}$ distribution (Fig. 21) shows a slight preference for GS96 at lower $E_{T}$ and GRV at larger $E_{T}$ (see also Adloff et al., 1998), but the experimental and theoretical uncertainties are still larger than the photon density variations. The H1 (Ahmed et al., 1995; Adloff et al., 2000) and ZEUS (Derrick et al., 1995b; Breitweg et al., 1998c) Collaborations were able to rule out the leading-order LAC1 and LAC3 photon densities in $x_{\gamma}^{\text {obs }}$ and $\bar{\eta}$ distributions, which are both sensitive to the photon densities (Forshaw and Roberts, 1993). The ZEUS distributions in $\eta_{2}$ (Breitweg et al., 1999c) do not allow any firm conclusions, since sizable hadronization corrections have not been included in the NLO predictions (Harris, Klasen, and Vossebeld, 1999). Dijet mass distributions have been measured up to 140 (Breitweg et al., 1999c) and $180 \mathrm{GeV}$ (Adloff et al., 2001b), respectively. They show no deviations from NLO QCD.

In photon-photon collisions, dijet transverse energy distributions have been measured with the cone algorithm by AMY (Kim et al., 1994), TOPAZ (Hayashii et al., 1993), and OPAL (Ackerstaff et al., 1997; Abbiendi et al., 1999). They are well described by NLO QCD (Kleinwort and Kramer, 1996a, 1997; Klasen, Kleinwort, and Kramer, 1998), as are the OPAL rapidity distributions (Abbiendi et al., 1999). The $k_{T}$ algorithm has been used by ALEPH (Barate et al., 2000) and OPAL (Abbi- 

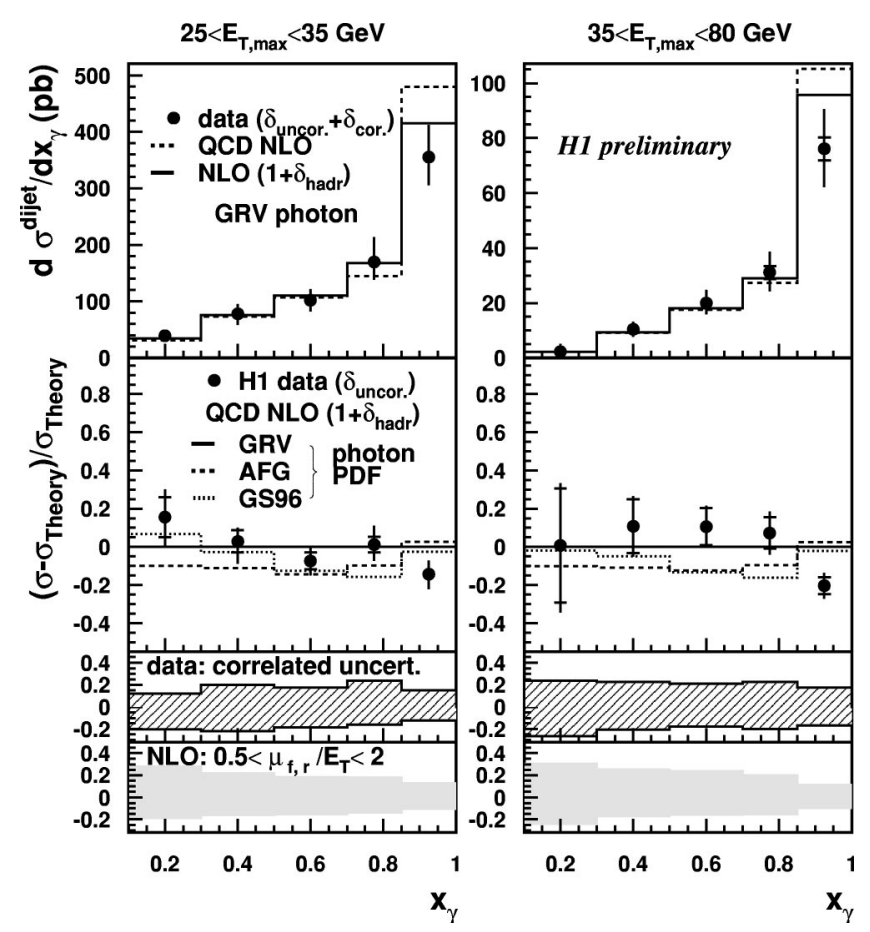

FIG. 21. Dependence of the dijet photoproduction cross section on the observed parton momentum fraction in the photon compared to preliminary $\mathrm{H} 1$ data. From Adloff et al., 2001b.

endi et al., 2001c; Wengler, 2002) to measure the transverse-energy and $x_{\gamma}^{\text {obs }}$ (only OPAL) distributions. The former is well described by NLO QCD. However, the infrared sensitivity of $x_{\gamma}^{\text {obs }}$ is reflected in Fig. 22 in the large fluctuation of the NLO prediction in the two

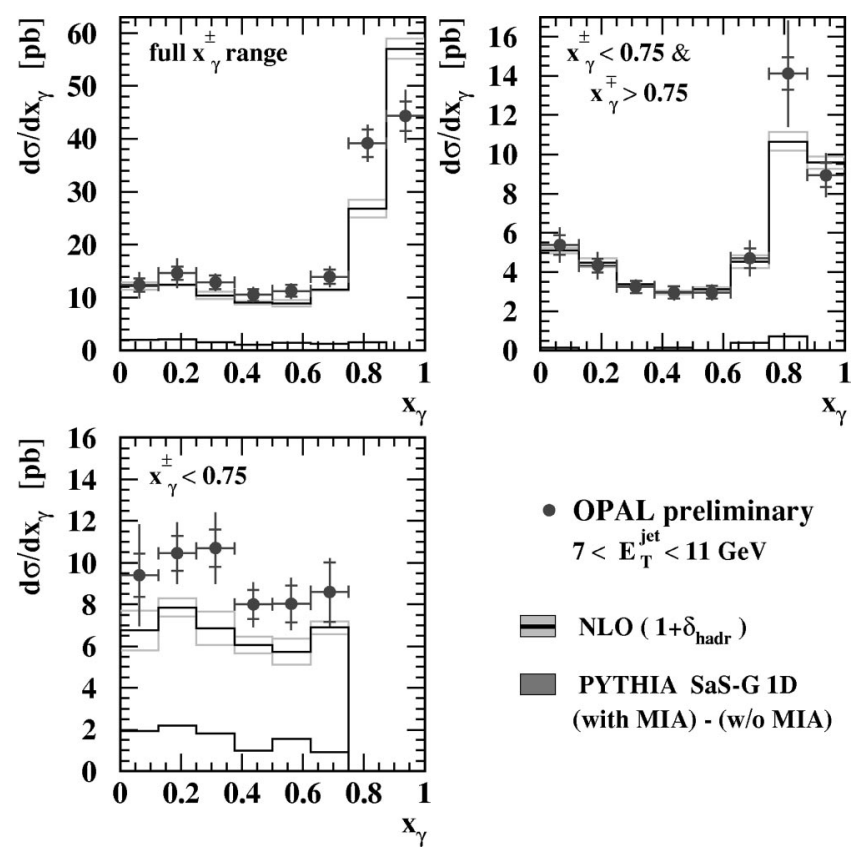

FIG. 22. Dependence of the dijet cross section in photonphoton collisions on the observed parton momentum fraction in the photon compared to preliminary OPAL data. From Wengler, 2002. highest bins. When both photons interact with low $x_{\gamma}^{\text {obs }}$, multiple parton interactions can lead to a larger observed cross section than predicted by next-to-leadingorder QCD with Glück-Reya-Vogt photon densities.

Higher center-of-mass energies may be reached at linear $e^{+} e^{-}$colliders like TESLA, ILC $(\sqrt{S}$ $=500-1000 \mathrm{GeV})$, or CLIC $(\sqrt{S}=1-3 \mathrm{TeV})$ or at a future $e p$ collider like THERA (TESLA $\times$ HERA, $\sqrt{S}$ $\sim 1 \mathrm{TeV}$ ). At linear colliders, the photoproduction cross section is enhanced due to beamstrahlung (see Sec. II.B) or laser backscattering (see Sec. II.C). Figure 23 demonstrates that tests of QCD and determinations of the photon structure could be considerably extended: Depending on the TESLA electron-beam energy (250-500 $\mathrm{GeV}$ ) and the collider mode ( $e p$ or $\gamma p$ ), the THERA range in the average transverse energy of the two jets would be increased by a factor of $2-3$ and the reach in $x_{\gamma}^{\text {obs }}$ by at least one order of magnitude. The reach in $x_{p}^{\text {obs }}$ is extended by about the same amount. It would thus become possible to check the determinations of the gluon density in the proton obtained in deep-inelastic scattering experiments and to measure the gluon density in the photon down to low values of $x$ (Klasen, 2001c; Wing, 2001). Similar studies have been performed for high-energy muon-proton collisions, although here bremsstrahlung is reduced and laser backscattering seems impossible (Klasen, 1997b).

\section{Three jets}

For $N$ massless jets, one can choose $3 N-4$ parameters that should span the multijet parameter space. They should also facilitate a simple interpretation within QCD and allow for a comparison of the $N-1$ jet to the $N$-jet cross section. In the case of $N=3$, the conventional choices are the three-jet mass $M_{123}=\sqrt{\left(p_{1}+p_{2}+p_{3}\right)^{2}}$ and four dimensionless parameters. The Dalitz energy fractions

$$
x_{i}=\frac{2 E_{i}}{M_{123}}
$$

specify how the available energy is shared among the three jets. They are ordered such that $x_{1}>x_{2}>x_{3}$. Since $x_{1}+x_{2}+x_{3}=2$, only $x_{1}$ and $x_{2}$ are linearly independent. The third and fourth parameters are the cosine of the scattering angle between the leading jet and the average beam direction $\vec{p}_{\mathrm{AV}}=\vec{p}_{a}-\vec{p}_{b}$, where the incoming parton $a$ is the one with the highest energy in the laboratory frame,

$$
\cos \theta_{1}=\frac{\vec{p}_{\mathrm{AV}} \vec{p}_{1}}{\left|\vec{p}_{\mathrm{AV}}\right|\left|\vec{p}_{1}\right|},
$$

and the angle between the three-jet plane and the plane containing the leading jet and the beam direction,

$$
\cos \psi_{1}=\frac{\left(\vec{p}_{1} \times \vec{p}_{\mathrm{AV}}\right)\left(\vec{p}_{2} \times \vec{p}_{3}\right)}{\left|\vec{p}_{1} \times \vec{p}_{\mathrm{AV}}\right|\left|\vec{p}_{2} \times \vec{p}_{3}\right|} .
$$

In the soft limit, where $E_{3} \rightarrow 0$ and $x_{1,2} \rightarrow 1, \cos \theta_{1}$ approaches the $2 \rightarrow 2$ center-of-mass scattering angle 

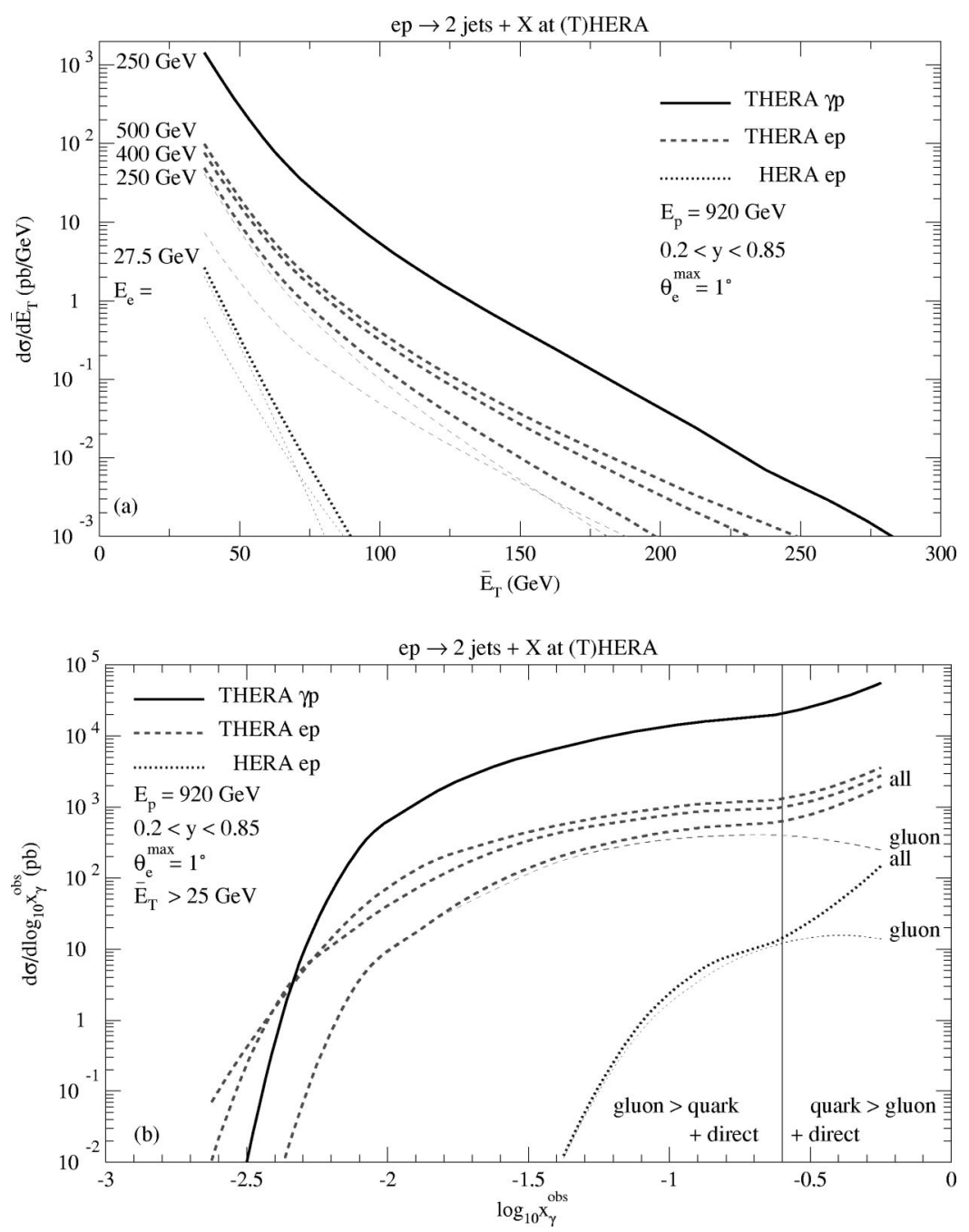

FIG. 23. Dijet photoproduction at a future THERA collider: (a) as a function of the average transverse energy of the two jets; (b) as a function of the observed parton momentum fraction in the photon. The thin lines in (a) show the separate contributions from the resolved (direct) processes for HERA and THERA with $E_{e}=250 \mathrm{GeV}$, which dominate at small (large) $\bar{E}_{T}$. $\cos \theta^{*}$, thus relating three-jet to dijet cross sections. The similarity of the distributions in the dijet and three-jet scattering angle distributions has already been discussed (see Fig. 19). Of course, the third jet must not be too soft (or the hard jets too hard) to avoid soft singularities that would have to be absorbed into the next-to-leadingorder dijet cross section. This can be achieved by a cut on $x_{1}$, e.g., $x_{1}<0.95$. However, since the energy of a jet is always larger than its transverse energy, a cut like $E_{T, 3}>5 \mathrm{GeV}$ already ensures the absence of soft singularities. The three jets also have to be well separated in phase space from each other and from the incident beams to avoid initial- and final-state collinear singularities. This is ensured by a cut like $\left|\cos \theta_{1}\right|<0.8$, by cuts on the pseudorapidities, and by the $k_{T}$ jet algorithm.

The three-jet photoproduction cross section

$$
\begin{aligned}
& \frac{d^{4} \sigma}{d x_{1} d x_{2} d \cos \theta_{1} d \psi_{1}} \\
& =\sum_{a, b} x_{a} f_{a / A}\left(x_{a}, M_{a}^{2}\right) x_{b} f_{b / B}\left(x_{b}, M_{b}^{2}\right) \frac{\overline{|\mathcal{M}|_{a b \rightarrow 123}^{2}}}{1024 \pi^{4}}
\end{aligned}
$$

has been calculated from the direct and resolved treelevel $2 \rightarrow 3$ processes by several groups with good mutual agreement (Baer, Ohnemus, and Owens, 1989a; Harris, Klasen, and Vossebeld, 1999; Klasen, 1999a, 1999b). Figure 24 shows the three-jet cross section at HERA as a function of the energy fractions of the (a) leading and (b) next-to-leading jet $x_{1}$ and $x_{2}$, normalized to the total cross section. The predictions from the $\mathcal{O}\left(\alpha \alpha_{s}^{2}\right)$ QCD matrix elements are rather similar to the pure phasespace distributions with constant matrix elements, but the ZEUS data (Breitweg et al., 1998e) slightly favor the QCD predictions. The dependence on the angle between the three-jet plane and the plane containing the leading jet and the average beam direction is shown in Fig. 24(c). The full QCD curve again agrees well with the data, as do the contributions from direct photons and quarks in the photon, whereas the pure phase space has a completely different shape.

Unfortunately, a full NLO calculation for three-jet photoproduction has not yet been performed. Such a calculation would allow for a determination of $\alpha_{s}$ from the ratio of three-jet to dijet cross sections and for a detailed study of the jet substructure. It could furthermore form the basis of a NNLO dijet calculation with reduced renormalization and factorization scale dependences. Higher-order corrections could also be taken into account approximately with resummation and 

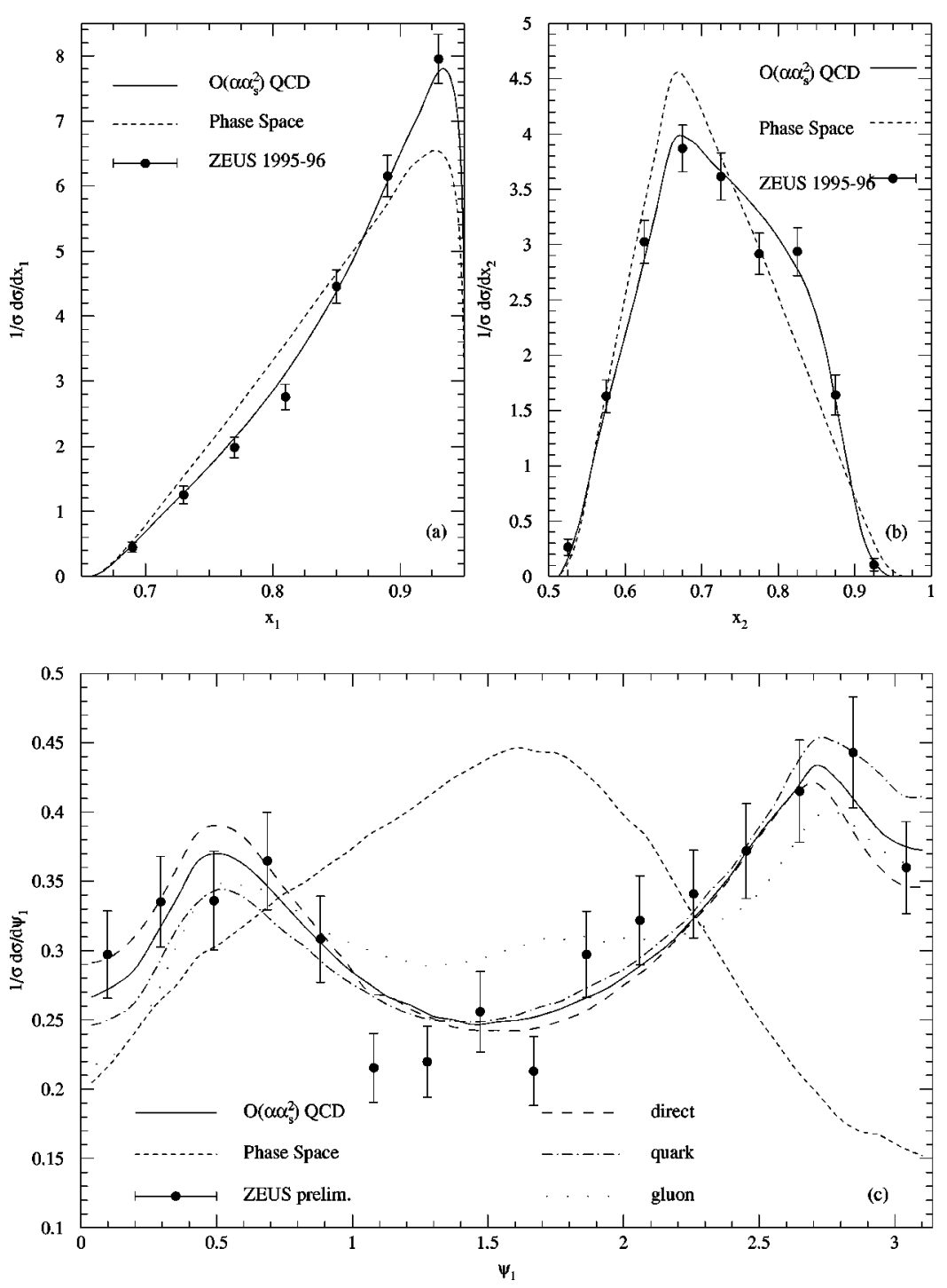

FIG. 24. Dependence of the three-jet cross section on (a) the energy fractions $x_{1}$, (b) $x_{2}$, and (c) the angle between the three-jet plane and the plane containing the leading jet and the average beam direction. The ZEUS data (Breitweg et al., 1998e) rule out the pure phase-space and pure gluon-initiated distributions. parton-shower techniques, leading eventually to more precise Monte Carlo generators for use by the experimental groups.

\section{E. Dijets with a leading neutron}

Recently H1 (Adloff et al., 2001c) and ZEUS (Breitweg et al., 2000c) presented data on dijet photoproduction with a leading neutron, which is dominated by slightly off-shell pion exchange and can be used to constrain the parton densities in the pion. These are not well known, particularly at low $x_{\pi}$ and in the sea-quark and gluon sectors. The pion structure carries important implications for the QCD confinement mechanism and the realization of symmetries like isospin in Nature. It is also of practical importance for the hadronic input to the photon structure at low scales (see Sec. III.C).

Assuming factorization, the photoproduction cross section for two jets with a leading neutron

$$
\begin{aligned}
& \frac{d^{3} \sigma}{d E_{T}^{2} d \eta_{1} d \eta_{2}} \\
& \quad=\sum_{a, b} x_{a} f_{a / e}\left(x_{a}, M_{\gamma}^{2}\right) x_{b} f_{b / p}\left(x_{b}, M_{\pi}^{2}\right) \frac{d \sigma}{d t}
\end{aligned}
$$

depends on the partonic cross section $d \sigma / d t$ [Eq. (4.10)] and on the parton densities in the electron $f_{a / e}[\mathrm{Eq}$. (4.16)] and proton,

$$
\begin{aligned}
f_{b / p}\left(x_{b}, M_{\pi}^{2}\right) \\
\quad=\int_{x_{b}}^{1} \frac{d\left(1-x_{n}\right)}{1-x_{n}} f_{b / \pi}\left(x_{\pi}, M_{\pi}^{2}\right) f_{\pi / p}\left(1-x_{n}, t^{\prime}\right) .
\end{aligned}
$$

The latter is a convolution of the parton densities in the pion $f_{b / \pi}$ with the pion flux in the $p \rightarrow n \pi$ transition

$$
\begin{aligned}
f_{\pi / p}\left(1-x_{n}, t^{\prime}\right)= & \frac{1}{4 \pi} \frac{g^{2}}{4 \pi} \frac{-t^{\prime}}{\left(m_{\pi}^{2}-t^{\prime}\right)^{2}} \\
& \times\left(1-x_{n}\right)^{1-2 \alpha_{\pi}^{\prime}\left(t^{\prime}-m_{\pi}^{2}\right)} \\
& \times\left[F\left(x_{n}, t^{\prime}\right)\right]^{2},
\end{aligned}
$$



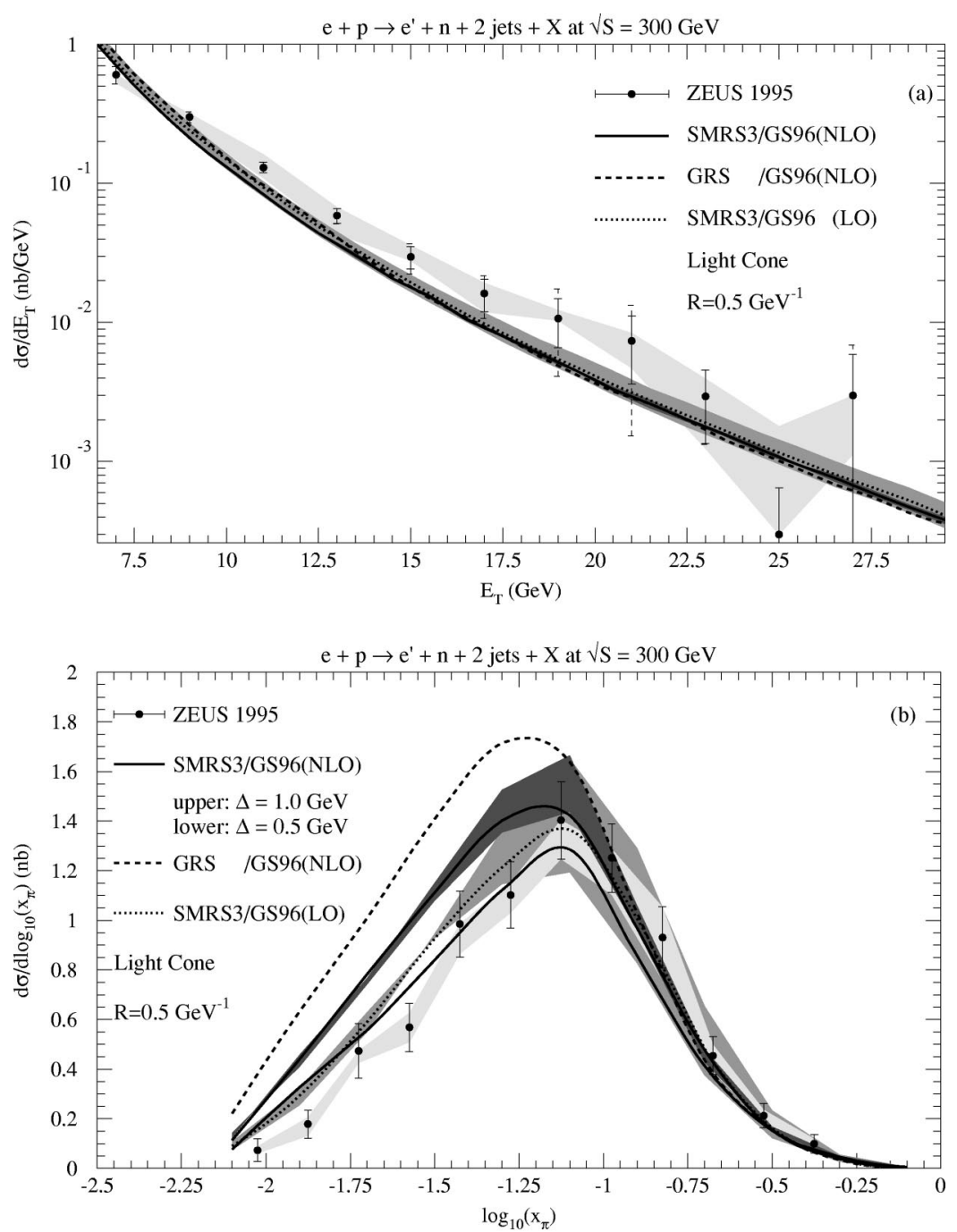

FIG. 25. Dependence of the dijet photoproduction cross section with a leading neutron (a) on the transverse jet energy, and (b) on the observed parton momentum fraction in the pion compared to ZEUS data (Breitweg et al., 2000c). The error bands show the theoretical scale uncertainty: medium, leading order; dark, NLO; light, the experimental energy scale uncertainty. where $1 /\left(m_{\pi}^{2}-t^{\prime}\right)^{2}$ is the squared pion propagator, $m_{\pi}$ is the pion mass, and $\left(1-x_{n}\right)^{1-2 \alpha_{\pi}^{\prime}\left(t^{\prime}-m_{\pi}^{2}\right)}$ accounts for the virtuality and possible Reggeization of the pion. In the ZEUS analysis, the momentum fraction of the leading neutron $x_{n}$ and the momentum transfer $t^{\prime}$ are restricted to $x_{n}>400 \mathrm{GeV} / 820 \mathrm{GeV}$ and $t^{\prime}=f\left(p_{T}\right)=-p_{T}^{2} / x_{n}-(1$ $\left.-x_{n}\right)\left(m_{n}^{2}-x_{n} m_{p}^{2}\right) / x_{n}$ with $p_{T}<x_{n} \cdot 0.66 \mathrm{GeV}$. The interaction term in the pion-nucleon Lagrangian leads to the numerator $-t^{\prime}$, and off-mass-shell effects of higher Fock states are modeled by a form factor

$F\left(x_{n}, t^{\prime}\right)=\left\{\begin{array}{l}\exp \left[b\left(t^{\prime}-m_{\pi}^{2}\right)\right], \quad[\text { exponential }] \\ \exp \left[R^{2}\left(t^{\prime}-m_{\pi}^{2}\right) /\left(1-x_{n}\right)\right], \quad[\text { light cone }] .\end{array}\right.$

Since the momentum transfer $t^{\prime}$ is small, Reggeization can be neglected $\left(\alpha^{\prime}=0\right)$, and a light-cone form factor with $R=0.5 \mathrm{GeV}^{-1}$ can be chosen in good agreement with recent determinations (Holtmann, Speth, and Szczurek, 1996; D'Alesio and Pirner, 2000). The pionnucleon coupling constant $g^{2} /(4 \pi)=14.17$ can be taken from a recent extraction from the GoldbergerMiyazawa-Oehme sum rule (Ericson, Loiseau, and Thomas, 2000).

In Fig. 25, leading-order and next-to-leading-order
QCD predictions (Klasen and Kramer, 2001; Klasen, 2001a, 2001b) with SMRS3 (Martin et al., 1992) and GRS (Glück, Reya, and Schienbein, 1999a) pion densities and GS96 photon densities are compared to the ZEUS measurements (Breitweg et al., 2000c). In the transverse energy distribution (a), the scale dependence is reduced considerably from leading order to next-toleading order. The normalization is sensitive to the chosen pion flux factor. If one includes the Regge trajectory $\left(\alpha^{\prime}=1 \mathrm{GeV}^{-2}\right)$ and omits the form factor, the cross section is reduced by $15 \%$. The distribution in the observed momentum fraction of the partons in the pion (b),

$$
x_{\pi}^{\mathrm{obs}}=\frac{E_{T_{1}} e^{\eta_{1}}+E_{T_{2}} e^{\eta_{2}}}{2\left(1-x_{n}\right) E_{p}}
$$

suffers from the fact that ZEUS applied equal cuts on $E_{T_{1}}, E_{T_{2}}>6 \mathrm{GeV}$ and $\eta_{1,2} \in[-2 ; 2]$, which had to be relaxed to $E_{T, 2}>6 \mathrm{GeV}-\Delta$ in the NLO calculation. The best fit is obtained with GS96 photon densities and SMRS3 pion densities, which have the lowest quark and gluon distributions, respectively, but hadronization corrections may play an important role at small $x_{\pi}$. 


\section{HADRON PRODUCTION}

In the last section it was demonstrated that jets provide an intuitive link with the partonic scattering processes, yield large rates in experimental measurements, and are well suited to determine the structure of the incoming photons, protons, or pions. However, they do not provide detailed information about the final-state hadronization, which can be better studied in the production of light and heavy hadrons and quark-antiquark bound states (quarkonia).

Early measurements of inclusive light-hadron production were performed in fixed-target collisions by the CERN NA14 experiment (Auge et al., 1986; Barate et al., 1986) and in photon-photon collisions at DESY PETRA (Berger et al., 1979; Brandelik et al., 1981). Heavy mesons were studied by the experiments CERN WA4 (Roudeau et al., 1980) and NA14/2 (Alvarez et al., 1992, 1993), Fermilab E687 (Frabetti et al., 1993, 1996; Moroni et al., 1994) and E691 (Anjos et al., 1989, 1990), DESY JADE (Bartel et al., 1987), and KEK AMY (Aso et al., 1995; Takashimizu et al., 1996), TOPAZ (Enomoto et al., 1994a, 1994b), and VENUS (Uehara et al., 1994, Ohyama et al., 1997).

In this section, the fragmentation of partons to light and heavy hadrons and quarkonia and their production in photon-hadron and photon-photon collisions will be discussed.

\section{A. Fragmentation}

As mentioned in Sec. IV.A, the Kinoshita-LeeNauenberg theorem guarantees that the soft and collinear singularities generated by real-particle emission beyond the leading order cancel against those generated by virtual-particle exchanges after summation over degenerate final states. In semi-inclusive final states, which contain identified particles, this cancellation is incomplete. Collinear $1 / \varepsilon$ poles multiplying the timelike partonic splitting functions $P_{j \leftarrow i}(x)$ in the transition functions remain:

$$
\begin{aligned}
\bar{\Gamma}_{j \leftarrow i}\left(x, M_{f}^{2}\right)= & \delta_{i j} \delta(1-x)-\frac{1}{\varepsilon} \frac{\alpha_{s}\left(\mu^{2}\right)}{2 \pi} \frac{\Gamma(1-\varepsilon)}{\Gamma(1-2 \varepsilon)} \\
& \times\left(\frac{4 \pi \mu^{2}}{M_{f}^{2}}\right)^{\varepsilon} P_{j \leftarrow i}(x)+\mathcal{O}\left(\varepsilon, \alpha_{s}^{2}\right) \\
= & \delta_{i j} \delta(1-x)-\left(\frac{1}{\varepsilon}-\gamma_{E}+\ln (4 \pi)+\ln \frac{\mu^{2}}{M_{f}^{2}}\right) \\
& \times \frac{\alpha_{s}\left(\mu^{2}\right)}{2 \pi} P_{j \leftarrow i}(x)+\mathcal{O}\left(\varepsilon, \alpha_{s}^{2}\right) .
\end{aligned}
$$

As in the case of parton distributions (see Sec. III), the factorization theorem (Altarelli et al., 1979; Baier and Fey, 1979; Ellis et al., 1979; Furmanski and Petronzio, 1980, 1982) allows these singularities to be absorbed in the $\overline{\mathrm{MS}}$ scheme into renormalized fragmentation functions,

$$
D_{H / i}\left(x, M_{f}^{2}\right)=\bar{D}_{H / i}(x)+\left[\bar{\Gamma}_{j \leftarrow i}\left(M_{f}^{2}\right) \otimes \bar{D}_{H / j}\right](x),
$$

where $x$ is the longitudinal momentum fraction of the hadron $H$ in the parton $i$ and $\bar{D}_{H / i}(x), \bar{D}_{H / j}(x)$ are the bare fragmentation functions. In this way one obtains important, yet incomplete, perturbative information about the confinement of unobservable quarks and gluons into observable hadrons. The fragmentation functions satisfy the sum rules

$$
\sum_{H} \int_{0}^{1} d x x D_{H / i}\left(x, M_{f}^{2}\right)=1,
$$

i.e., the momentum of the parton $i$ must be conserved after hadronization into all available hadrons $H$. Fragmentation functions constitute only the leading-twist contributions to semi-inclusive hadron production in the operator product expansion. Higher-twist, nonfactorizable operators can also contribute to the hadronization process. However, in the transverse/longitudinal and total $e^{+} e^{-}$cross sections they are suppressed by additional factors of $1 / Q$ and $1 / Q^{2}$, respectively, and thus are usually negligible (Dasgupta and Webber, 1997).

The evolution of the fragmentation functions can be calculated by taking the logarithmic derivative of Eq. (5.2) with respect to the scale $M_{f}^{2} \equiv Q^{2}$. This leads to the coupled homogeneous evolution equations (Georgi and Politzer, 1978; Altarelli et al., 1979; Baier and Fey, 1979)

$$
\begin{aligned}
\frac{d D_{H / q}\left(Q^{2}\right)}{d \ln Q^{2}}= & \frac{\alpha_{s}\left(Q^{2}\right)}{2 \pi}\left[P_{q \leftarrow q} \otimes D_{H / q}\left(Q^{2}\right)\right. \\
& \left.+P_{g \leftarrow q} \otimes D_{H / g}\left(Q^{2}\right)\right], \\
\frac{d D_{H / g}\left(Q^{2}\right)}{d \ln Q^{2}}= & \frac{\alpha_{s}\left(Q^{2}\right)}{2 \pi}\left[P_{q \leftarrow g} \otimes D_{H / q}\left(Q^{2}\right)\right. \\
& \left.+P_{g \leftarrow g} \otimes D_{H / g}\left(Q^{2}\right)\right],
\end{aligned}
$$

where the timelike splitting functions $P_{j \leftarrow i}$ are identical to the spacelike splitting functions in leading order (Altarelli and Parisi, 1977), but differ in next-to-leading order (Curci, Furmanski, and Petronzio, 1980; Furmanski and Petronzio, 1980; Floratos, Kounnas, and Lacaze, 1981).

The evolution equations unfortunately cannot be solved analytically, and for light hadrons an initial distribution similar to the form $D_{H / i}\left(x, Q_{0}^{2}\right)=N x^{\alpha}(1-x)^{\beta}$ has to be assumed at a low starting scale $Q_{0}$. The free constants $N, \alpha$, and $\beta$ are then fitted to experimental data, usually from the process $e^{+} e^{-} \rightarrow \gamma^{*}(q)$ $\rightarrow H\left(p_{H}\right) X$, where the initial-state and the total centerof-mass energy $Q^{2}=q^{2}$ are uniquely fixed. In the $\overline{\mathrm{MS}}$ scheme, the normalized NLO semi-inclusive hadron cross section is given by (Altarelli et al., 1979; Baier and Fey, 1979)

$$
\begin{aligned}
\frac{1}{\sigma_{\mathrm{tot}}} \frac{d \sigma\left(Q^{2}\right)}{d x}= & \sum_{q} 2\left(D_{H / q}\left(Q^{2}\right)+\sum_{i=T, L} \frac{\alpha_{s}\left(Q^{2}\right)}{2 \pi} \times\left[C_{q}^{i}\right.\right. \\
& \left.\left.\otimes D_{H / q}\left(Q^{2}\right)+C_{g}^{i} \otimes D_{H / g}\left(Q^{2}\right)\right]\right),
\end{aligned}
$$

where 
TABLE X. Parametrizations of various hadron fragmentation functions.

\begin{tabular}{|c|c|c|c|c|c|c|}
\hline Group & Year & Hadron & $\begin{array}{c}Q_{0}^{2} \\
\left(\mathrm{GeV}^{2}\right)\end{array}$ & $\begin{array}{l}\text { Factor. } \\
\text { scheme }\end{array}$ & $N_{f}$ & $\begin{array}{l}\Lambda_{\mathrm{MS}}^{N_{f}=4} \\
(\mathrm{MeV})\end{array}$ \\
\hline BEP & 1979 & $\pi^{ \pm, 0}, K^{ \pm}, p$ & 25.0 & LO & 3 & $450 / 600$ \\
\hline AKL & 1983 & $\pi^{ \pm}, K^{ \pm}$ & 25.0 & LO & 3 & $300 / 400$ \\
\hline $\mathrm{GR}+$ & $1993 / 5$ & $\pi^{ \pm, 0}, \eta, K^{ \pm, 0}$ & 900 & $\overline{\mathrm{MS}}$ & 5 & $269 / 319$ \\
\hline NW & 1994 & $h^{ \pm}$ & $m_{Z}^{2}$ & $\overline{\mathrm{MS}}$ & 5 & 344 \\
\hline \multirow[t]{4}{*}{ BKK } & 1995 & $\pi^{ \pm}, K^{ \pm}$ & 2.00 & LO & 5 & 190 \\
\hline & & & & $\overline{\mathrm{MS}}$ & & 190 \\
\hline & $1995 / 8$ & $\pi^{ \pm}, K^{ \pm, 0}$ & 2.00 & $\mathrm{LO}$ & 5 & 146 (fit) \\
\hline & & $D^{* \pm}, B^{+, 0}$ & $4 m_{c, b}^{2}$ & $\overline{\mathrm{MS}}$ & & 317 (fit) \\
\hline CGRT & 1997 & $D^{0}, D^{* 0}$ & $m_{c}^{2}$ & $\overline{\mathrm{MS}}$ & 5 & 151 \\
\hline \multirow[t]{2}{*}{ KKP } & 2000 & $\pi^{ \pm}, K^{ \pm}, p$ & 2.00 & LO & 5 & 121 (fit) \\
\hline & & & & $\overline{\mathrm{MS}}$ & & 299 (fit) \\
\hline \multirow[t]{2}{*}{ Kretzer } & 2000 & $\pi^{ \pm}, K^{ \pm}, h^{ \pm}$ & 0.26 & LO & 5 & 175 \\
\hline & & & 0.40 & $\overline{\mathrm{MS}}$ & & 246 \\
\hline BFGW & 2001 & $h^{ \pm}$ & 2.00 & $\overline{\mathrm{MS}}$ & 5 & 300 \\
\hline
\end{tabular}

$$
\sigma_{\mathrm{tot}}=N_{C} \sum_{q} e_{q}^{2} \sigma_{0}\left(1+\frac{\alpha_{s}\left(Q^{2}\right)}{\pi}\right)
$$

is the total hadronic cross section, $x=2\left(p_{H} \cdot q\right) / Q^{2}$ is the fraction of the center-of-mass energy transferred to the observed hadron $H$, and $\sigma_{0}=4 \pi \alpha^{2} /\left(3 Q^{2}\right)$ is the total cross section for $e^{+} e^{-} \rightarrow \mu^{+} \mu^{-}$. At larger $Q^{2}$, the couplings and propagators in Eqs. (5.5) and (5.6) are modified due to additional $Z$-boson exchange. The timelike transverse $(T)$ and longitudinal $(L)$ Wilson coefficients are given by (Altarelli et al., 1979)

$$
\begin{aligned}
C_{q, T}^{T}= & C_{F}\left[\frac{3}{2}(1-x)-\frac{3}{2} \frac{1}{(1-x)_{+}}+2 \frac{1+x^{2}}{1-x} \ln x\right. \\
& \left.+\left(1+x^{2}\right)\left(\frac{\ln (1-x)}{1-x}\right)_{+}+\left(\frac{2 \pi^{2}}{3}-\frac{9}{2}\right) \delta(1-x)\right], \\
C_{q, L}^{T}= & C_{F}, \\
C_{g, T}^{T}= & C_{F}\left(\frac{1+(1-x)^{2}}{x} \ln \left[x^{2}(1-x)\right]-\frac{2(1-x)}{x}\right), \\
C_{g, L}^{T}= & C_{F} \frac{2(1-x)}{x} .
\end{aligned}
$$

The transverse cross section starts at $\mathcal{O}\left(\alpha_{s}^{0}\right)$, while the longitudinal cross section starts only at $\mathcal{O}\left(\alpha_{s}\right)$. Thus in next-to-leading order the longitudinal $\mathcal{O}\left(\alpha_{s}^{2}\right)$ Wilson coefficients have to be included for longitudinal cross sections, but not for total cross sections (Rijken and van Neerven, 1996, 1997).

For a long time, only leading-order fragmentation functions for charged pions, kaons, and protons were available, which had been fitted to $e^{+} e^{-}$data from MARK I (Baier, Engels, and Petersson, 1979). These were subsequently updated using deep-inelastic data from EMC (Anselmino, Kroll, and Leader, 1983). Improved leading-order sets for pions, $\eta$ 's, kaons, and $D$ mesons were obtained by comparing HERWIG Monte Carlo predictions in the next-to-leading logarithmic approximation with $e^{+} e^{-}$data from TPC, PETRA, and later LEP (Greco and Rolli, 1993, 1995; Chiappetta et al., 1994; Nason and Webber, 1994; Greco, Rolli, and Vicini, 1995; Cacciari et al., 1997). Recently several independent sets have been obtained by a full NLO evolution of a nonperturbative input at low starting scales and fits to precise $e^{+} e^{-}$data from MARK II, TPC, PETRA, AMY, SLD, and LEP (Binnewies, Kniehl, and Kramer, 1995a, 1995b, 1996a, 1997, 1998a, 1998b; Kniehl, Kramer, and Pötter, 2000a; Kretzer, 2000; Bourhis et al., 2001). These data make it possible to simultaneously determine $\alpha_{s}$ with a precision that is competitive with the world average (Binnewies, Kniehl, and Kramer, 1995b; Kniehl, Kramer, and Pötter, 2000b). The extracted value of $\alpha_{s}$ could in principle be correlated with the size of power corrections (Nason and Webber, 1994), but the effect was found to be negligible (Kniehl, Kramer, and Pötter, 2000b). According to the factorization theorem, fragmentation functions should be universal, i.e., process independent. The validity of this assumption has been verified by comparing inclusive hadron cross sections with $e^{+} e^{-}$data not used for the fits at different scales $Q^{2}$ and with different hard scattering processes like photon-photon, photon-hadron, and hadron-hadron collisions (Binnewies, Kniehl, and Kramer, 1995b, 1996b, 1997, 1998a, 1998b; Kniehl, Kramer, and Pötter, 2001). The parametrizations of the various fragmentation functions discussed above are compared in Table X. From Fig. 26 one observes that the three NLO fits (Kniehl, Kramer, and Pötter; Kretzer; and Bourhis et al.) are very similar for the fragmentation of light quarks and all quarks, except for the theoretically unreliable small- $x$ 


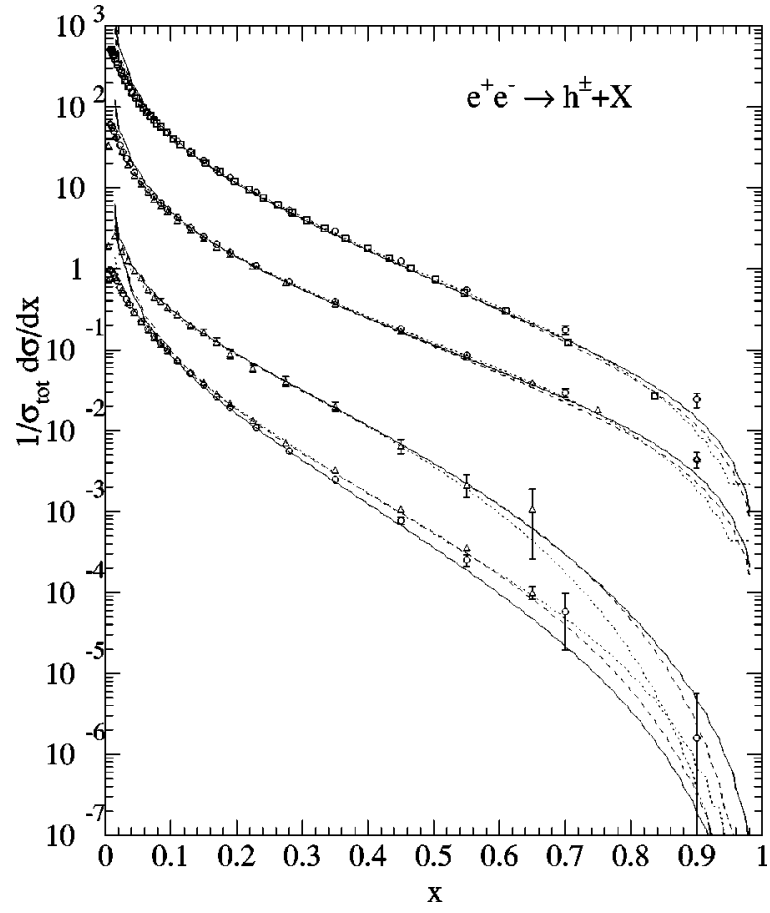

FIG. 26. Dependence of the normalized inclusive hadron cross section at $\sqrt{S}=91.2 \mathrm{GeV}$ on the scaled hadron momentum $x$. Data from DELPHI $(\triangle, 1991-1993 ; \bigcirc, 1994 ; \square$, SLD) are compared with the NLO fragmentation functions of Kniehl, Kramer, and Pötter (solid curve), Kretzer (dotted curve), and Bourhis, Fontannaz, et al. (dashed curve). Contributions from $b, c$, light, and all quarks (from bottom to top) are rescaled by factors of $1 / 5$ relative to the nearest upper distribution. From Kniehl, Kramer, and Pötter, 2001.

region $(x \leqslant 0.1)$ and the experimentally badly constrained large- $x$ region $(x \geqslant 0.8)$. For the fragmentation of $c$ and $b$ quarks there are also differences at intermediate values of $x$. In the case of the $b$ quarks the discrep- ancy is due to the fact that two incompatible data sets from DELPHI (1991-1993 and 1994 data) were used in the fits: Table XI shows that it is not possible to obtain good values of $\chi^{2}$ per degree of freedom, $\chi_{\mathrm{DF}}^{2}$, simultaneously for both data samples. Otherwise all three NLO fits give satisfactory values of $\chi_{\mathrm{DF}}^{2}$.

While the masses of light quarks and gluons can always be safely neglected in the massless evolution approach, the masses $m_{h}$ of the heavier charm and bottom quarks $h$ may become comparable to the physical scale $Q$. The even heavier top quark decays before it hadronizes. Bjorken (1978) argued that attaching a light antiquark $\bar{q}$ to a heavy quark $h$ should decelerate the latter only slightly, so that it carries almost the same energy as the hadron $H=(h \bar{q})$. As a consequence, a light-quark fragmentation function proposed by Field and Feynman (1978), which was peaked at rather low $x$, was modified (Ali, Körner, et al., 1980; Ali, Kramer, et al., 1980). Later Peterson et al. (1983) expanded the energies in the fragmentation amplitude $1 /\left(E_{H}-E_{h}+E_{\bar{q}}\right)$ about the transverse particle masses with the result

$$
D_{H / h}(x)=\frac{N}{x\left[1-1 / x-\varepsilon_{h} /(1-x)\right]^{2}},
$$

where $\varepsilon_{h} \propto m_{q}^{2} / m_{h}^{2}$ is a free parameter and $N$ is a normalization factor constrained by the momentum sum rule, Eq. (5.3). This fragmentation function peaks at $x=1$ $-\sqrt{\varepsilon_{h}}$ and has a width of $\sqrt{\varepsilon_{h}}$ (Bodwin and Harris, 2001). Alternative forms have been proposed by Kartvelishvili, Likhoded, and Petrov (1978), Bowler (1981), Andersson et al. (1983), and Suzuki (1986). Using heavy-quark effective theory, Braaten, Cheung, and Yuan (1993a) calculated the $\mathcal{O}\left(\alpha_{s}^{2}\right)$ fragmentation function

TABLE XI. $\chi_{\mathrm{DF}}^{2}$ values obtained in comparisons of the NLO fragmentation functions by Kniehl, Kramer, and Potter (KKP), Kretzer, and Bourhis, Fontannaz, et al. (BFGW) to $e^{+} e^{-}$data with $x$ $>0.1$ at different center-of-mass energies. Data samples, which did not enter the respective fits, are marked by asterisks (Kniehl, Kramer, and Pötter, 2001).

\begin{tabular}{|c|c|c|c|c|c|c|}
\hline \multirow{2}{*}{$\begin{array}{l}\text { Energy } \\
(\mathrm{GeV})\end{array}$} & \multirow[b]{2}{*}{ Flavor } & \multirow[b]{2}{*}{ Experiment } & \multicolumn{3}{|c|}{ FF set } & \multirow{2}{*}{$\begin{array}{l}\text { No. of } \\
\text { points }\end{array}$} \\
\hline & & & KKP & Kretzer & BFGW & \\
\hline \multirow[t]{3}{*}{29} & $u d s$ & $\mathrm{TPC}$ & $0.178^{*}$ & 0.159 & $0.167 *$ & 7 \\
\hline & $c$ & & $0.876^{*}$ & 0.911 & $0.923^{*}$ & 7 \\
\hline & $b$ & & $2.23 *$ & 1.21 & $1.14^{*}$ & 7 \\
\hline \multirow[t]{7}{*}{91.2} & all & DELPHI 94 & 1.28 & $1.51^{*}$ & 1.49 & 12 \\
\hline & & SLD & 1.32 & 0.370 & 0.421 & 21 \\
\hline & $u d s$ & DELPHI 91-3 & $3.17 *$ & $0.990^{*}$ & 1.95 & 13 \\
\hline & & DELPHI 94 & 0.201 & $0.588^{*}$ & $1.00 *$ & 12 \\
\hline & $c$ & DELPHI 91-3 & $0.473^{*}$ & $0.388^{*}$ & 0.401 & 11 \\
\hline & $b$ & DELPHI 91-3 & $28.9 *$ & $0.887^{*}$ & 1.03 & 12 \\
\hline & & DELPHI 94 & 0.433 & $9.14^{*}$ & 8.74 & 12 \\
\hline 189 & all & OPAL & $0.568 *$ & $0.250^{*}$ & $0.414^{*}$ & 11 \\
\hline
\end{tabular}




$$
\begin{aligned}
D_{B_{c} / b}\left(x, m_{b}+2 m_{c}\right) \\
=N \frac{r x(1-x)^{2}}{[1-(1-r) x]^{6}}[6-18(1-2 r) x \\
\quad+\left(21-74 r+68 r^{2}\right) x^{2}-2(1-r)(6-19 r \\
\left.\left.\quad+18 r^{2}\right) x^{3}+3(1-r)^{2}\left(1-2 r+2 r^{2}\right) x^{4}\right]
\end{aligned}
$$

for $B_{c}$ mesons, which depends on the mass ratio $r$ $=m_{c} /\left(m_{b}+m_{c}\right)$. Like the nonperturbative fragmentation functions discussed above, this result can also be used to parametrize the input at the starting scale $Q_{0}$ for heavy-light mesons with the normalization $N$ and $r$ as free parameters (Braaten, Cheung, et al., 1995; Binnewies, Kniehl, and Kramer, 1998b). Equation (5.9) peaks at large $x(x \simeq 0.8)$ and vanishes correctly like $(1-x)^{2}$ as $x \rightarrow 1$, whereas a previous perturbative calculation by Collins and Spiller (1985) only vanishes like $(1-x)$ and fails to describe recent ALEPH data (Heister et al., 2001).

Matching a massless NLO calculation for $e^{+} e^{-} \rightarrow h X$ in the $\overline{\mathrm{MS}}$ scheme to a NLO calculation with $m_{h}$ as a regulator for the collinear singularity, Mele and Nason (1991) calculated the functions

$$
\begin{aligned}
& D_{H / h}\left(x, Q^{2}\right)= \delta(1-x)+\frac{\alpha_{s}\left(Q^{2}\right) C_{F}}{2 \pi}\left[\frac { 1 + x ^ { 2 } } { 1 - x } \left(\ln \frac{Q^{2}}{m_{h}^{2}}\right.\right. \\
&-2 \ln (1-x)-1)]_{+}, \\
& D_{H / g}\left(x, Q^{2}\right)= \frac{\alpha_{s}\left(Q^{2}\right) T_{R}}{2 \pi}\left[x^{2}+(1-x)^{2}\right] \ln \frac{Q^{2}}{m_{h}^{2}}, \\
& D_{H / q, \bar{q}, \bar{h}}\left(x, Q^{2}\right)=\mathcal{O}\left(\alpha_{s}^{2}\right),
\end{aligned}
$$

which reproduce the massive cross section when convolved with the massless hard scattering cross section at the scale $Q$. For scales $Q \gg m_{h}$, however, the logarithms in Eqs. (5.10) become large and have to be resummed. In this case, these equations can be interpreted as boundary conditions at a starting scale $Q_{0}$ and can be evolved to the scale $Q$. Both approaches were found to give similar results in photoproduction (Cacciari and Greco, 1996) and $e^{+} e^{-}$annihilation (Nason and Oleari, 2000), but they yielded a satisfactory description of the data only when an additional nonperturbative input was included, either of the form $D_{H / i}\left(x, Q_{0}^{2}\right)=N x^{\alpha}(1-x)^{\beta}$ (Cacciari et al., 1997) or of the form in Eq. (5.8) (Cacciari and Greco, 1997; Nason and Oleari, 1999, 2000). An alternative solution consists in adding Eqs. (5.10) to the massless cross-section calculation with the interpretation of a change of factorization scheme (Kniehl, Kramer, and Spira, 1997; Binnewies, Kniehl, and Kramer, 1998a; Kramer, 1999). The fitted values of the nonperturbative input depend, of course, on the treatment of the perturbative component.

The fragmentation of quarks and gluons into bound states of heavy quarks and antiquarks $\mathcal{Q}=(h \bar{h})$ is based on the general factorization analysis of Bodwin, Braaten, and Lepage (1995). It allows for the fragmen- tation functions of quarkonia $\mathcal{Q}$ to be factorized into calculable short-distance coefficients $d_{i}^{n}\left(x, Q^{2}\right)$, which describe the production rate of a quark-antiquark pair with quantum numbers $n=\left[\underline{a},{ }^{2 S+1} L_{J}\right]$ within a region of size $1 / m_{h}$, and long-distance operator matrix elements $\left\langle O^{\mathcal{Q}}(n)\right\rangle$, which contain the nonperturbative dynamics responsible for the formation of the bound state $\mathcal{Q}$ from the state $n$,

$$
D_{\mathcal{Q} / i}\left(x, Q^{2}\right)=\sum_{n} d_{i}^{n}\left(x, Q^{2}\right)\left\langle O^{\mathcal{Q}}(n)\right\rangle, \quad i \in\{q, h, g\} .
$$

The size of the operator matrix elements may be estimated by how they scale with the relative velocity $v$ of the quarks inside the quarkonium state (Lepage et al., 1992). In the case of the charmonium state $J / \Psi$ the leading operator matrix element

$$
\left\langle O_{1}^{J / \Psi}\left({ }^{3} S_{1}\right)\right\rangle=\frac{9}{2 \pi}\left|R_{J / \Psi}(0)\right|^{2}
$$

is related to the $J / \Psi$ radial wave function at the origin $R_{J / \Psi}(0)$ (Braaten, Fleming, and Yuan, 1996). The subleading operator-matrix elements $\left\langle O_{8}^{J / \Psi}\left({ }^{3} S_{1}\right)\right\rangle$, $\left\langle O_{8}^{J / \Psi}\left({ }^{1} S_{0}\right)\right\rangle$, and $\left\langle O_{8}^{J / \Psi}\left({ }^{3} P_{J}\right)\right\rangle$ follow at the relative order $\mathcal{O}\left(v^{4}\right)$. Of particular importance is the transverse gluon coefficient (Braaten and Yuan, 1994; Ma, 1995; Braaten and Lee, 2000)

$$
\begin{aligned}
d_{g}^{\left[8,{ }^{3} S_{1}\right]}\left(x, Q^{2}\right)= & \frac{\pi \alpha_{s}\left(Q^{2}\right)}{24 m_{h}^{3}}\left(\delta(1-x)+\frac{\alpha_{s}\left(Q^{2}\right)}{\pi}\right. \\
& \times\left\{\left[\beta_{0}\left(\ln \frac{Q}{2 m_{h}}+\frac{13}{6}\right)+\frac{2}{3}-\frac{\pi^{2}}{2}+8 \ln 2\right.\right. \\
& \left.+6 \ln ^{2} 2\right] \delta(1-x)+\left(\ln \frac{Q}{2 m_{h}}-\frac{1}{2}\right) \\
& \times P_{g \leftarrow g}(x)+6\left(2-x+x^{2}\right) \ln (1-x) \\
& \left.\left.-\frac{6}{x}\left(\frac{\ln (1-x)}{1-x}\right)\right\}\right)
\end{aligned}
$$

since it starts already at $\mathcal{O}\left(\alpha_{s}\right)$, while the gluon coefficient for the leading operator matrix element $\left\langle O_{1}^{J / \Psi}\left({ }^{3} S_{1}\right)\right\rangle$ starts only at $\mathcal{O}\left(\alpha_{s}^{3}\right)$ (Braaten and Yuan, 1995). Since in the charmonium system $v^{2} \simeq \alpha_{s}\left(m_{c}^{2}\right)$ $\simeq 0.3$ (Quigg and Rosner, 1979), the $\left\langle O_{8}^{J / \Psi}\left({ }^{3} S_{1}\right)\right\rangle$ contribution is no longer suppressed with respect to the $\left\langle O_{1}^{J / \Psi}\left({ }^{3} S_{1}\right)\right\rangle$ contribution. The perturbative coefficient for the fragmentation of heavy quarks into the leading $\left[1,{ }^{3} S_{1}\right]$ state is of $\mathcal{O}\left(\alpha_{s}^{2}\right)$ (Braaten, Cheung, and Yuan, 1993b; Ma, 1994).

For the $P$-wave $\chi_{c, J}$ states, the coefficients of the leading operator matrix elements $\left\langle O_{1}^{\chi_{c, J}}\left({ }^{3} P_{J}\right)\right\rangle$ with $J$ $=0,1,2$ start at $\alpha_{s}^{2}$ for both gluons (Braaten and Yuan, 1994; Ma, 1995) and quarks (Ma, 1996a). The coefficients for the subleading operator matrix elements $\left\langle O_{8}^{\chi_{c, J}}\left({ }^{3} S_{1}\right)\right\rangle$ start at $\mathcal{O}\left(\alpha_{s}\right)$ for gluons (see above) and 


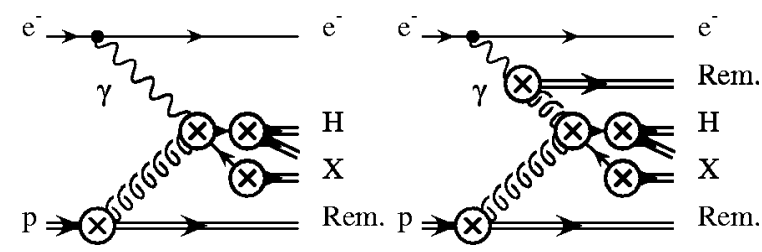

FIG. 27. Factorization of photon-hadron scattering into hadrons.

$\mathcal{O}\left(\alpha_{s}^{2}\right)$ for quarks (Ma, 1996a), as do the quark (Braaten, Cheung, and Yuan, 1993b; Ma, 1994) and gluon (Braaten and Yuan, 1993; Ma, 1994) coefficients for the leading operator matrix element in $\eta_{c}$ production $\left\langle O_{1}^{\eta_{c}}\left({ }^{1} S_{0}\right)\right\rangle$.

\section{B. Light hadrons}

Like jets, hadrons can be produced in direct, singleresolved, and double-resolved photon-photon scattering and in direct and resolved photon-hadron scattering. As can be seen in Fig. 27 for the photoproduction case, there is a new factorization in the final state for the transition of partons $c$ into observed hadrons $H$, which is described by fragmentation functions $D_{H / c}$. In the hadronic cross section

$$
\begin{aligned}
\frac{d^{2} \sigma}{d p_{T}^{2} d y}= & \sum_{a, b, c} \int d x_{a} d x_{b} \frac{d z}{z^{2}} f_{a / A}\left(x_{a}, M_{a}^{2}\right) f_{b / B}\left(x_{b}, M_{b}^{2}\right) \\
& \times D_{H / c}\left(z, M_{c}^{2}\right) \frac{d \sigma}{d t}
\end{aligned}
$$

the fragmentation function is convolved at a factorization scale $M_{c}$ with the partonic cross section Eq. (4.10) and the initial-state parton densities $f_{a / A}$ and $f_{b / B}$, which were already encountered in the jet production case.

The potential of inclusive hadron production for distinguishing the direct and resolved contributions was stressed in early leading-order calculations for photonphoton (Brodsky et al., 1979) and photon-hadron scattering (Fontannaz et al., 1980). For light hadrons, the calculation of Born, virtual loop, and real emission processes proceeds as described in Sec. IV.B. There is, however, an important difference. Since the degeneracy of the final state is incomplete due to the observation of an identified hadron, the collinear singularity associated with the real emission of a massless parton 3 from the observed final parton line $2 *$,

$$
\left|\mathcal{M}^{F}\right|_{a b \rightarrow 123}^{2}=-\frac{1}{\varepsilon} P_{2 \leftarrow 2 *}(x)\left|\mathcal{M}^{B}\right|_{a b \rightarrow 12 *}^{2}+\mathcal{O}\left(\varepsilon^{0}\right),
$$

remains uncanceled and has to be factorized into the fragmentation function (see Sec. V.A). NLO calculations based on the formalism of Ellis et al. (1980) have been performed for direct (Khalafi, Landshoff, and Stirling, 1983; Aurenche, Baier, et al., 1985a; Gordon, 1994), single-resolved (Aurenche, Baier, et al., 1984b, 1987; Gordon, 1994), and double-resolved (Aversa et al., 1989) light-hadron production, and their dependences on the renormalization and factorization scales were found to be considerably reduced with respect to the leadingorder calculations (Borzumati, Kniehl, and Kramer, 1993; Gordon, 1994; Kniehl and Kramer, 1994; Greco, Rolli, and Vicini, 1995). The NLO calculations were then used to demonstrate the universality of NLO fragmentation functions for charged hadrons, neutral pions, and neutral kaons (Binnewies, Kniehl, and Kramer, 1995b, 1996a, 1996b; Binnewies, 1997; Kniehl, 1997; Kniehl, Kramer, and Pötter, 2001) by comparing them with photoproduction data from H1 (Abt et al., 1994; Linsel, 1995; Adloff et al., 1997, 1999a) and ZEUS (Derrick et al., 1995c; Breitweg et al., 1998f) and photonphoton data from TASSO (Brandelik et al., 1981), MARK II (Cords et al., 1993), L3 (Achard et al., 2001), and OPAL (Ackerstaff et al., 1999).

In Fig. 28, NLO calculations of the inclusive chargedhadron photoproduction cross section at HERA with AFG photon and CTEQ5M (Lai et al., 2000) proton densities and three different choices of the scale factor $\xi=\mu / p_{T}=M_{\gamma} / p_{T}=M_{p} / p_{T}$ (Kniehl, Kramer, and Pötter, 2001) are compared to H1 data (Adloff et al., 1999a). The transverse momentum spectrum (top) agrees well with the data in shape and normalization. The rapidity spectrum (bottom) is sensitive to variations of the photon densities (AFG, GRV, or GS96; see Tables IV and $\mathrm{V})$, particularly for the lower $p_{T}$ cut, but the scale uncertainty is still large.

A similar comparison is made in Fig. 29 for the photoproduction of neutral kaons. Due to the larger kaon mass, the massless NLO calculation describes the measured $p_{T}$ spectrum (top) only at larger $p_{T} \quad\left(p_{T}\right.$ $>1.5 \mathrm{GeV})$. The cut on $p_{T}$ for the rapidity spectrum (bottom, $p_{T}>2 \mathrm{GeV}$ ) may still be too low for quantitative conclusions, but the potential to constrain $f_{g / \gamma}$ and $D_{K^{0 / g}}$ is clearly visible.

NLO charged-hadron cross sections in photon-photon collisions (Kniehl, Kramer, and Pötter, 2001) are shown in Fig. 30 and compared to OPAL data (Ackerstaff et al., 1999). Apart from the lowest $p_{T}$ points, which are below the starting scale of the fragmentation functions, the massless calculations describe the transverse momentum spectra (top) quite well. The scale variations in the rapidity spectra (bottom) are again significantly larger than the experimental errors and the sensitivity to the photon structure (not shown).

\section{Heavy hadrons}

If the transverse momentum $p_{T}$ of a produced hadron is much larger than its mass, the formalism described in the last section can also be applied to the production of heavy hadrons. The heavy quark is then considered to be one of the active flavors in the evolution of parton densities (see Sec. III) and fragmentation functions (see Sec. V.A), and the heavy-quark mass $m_{h}$ is neglected in the hard scattering cross section. It is only kept as the 

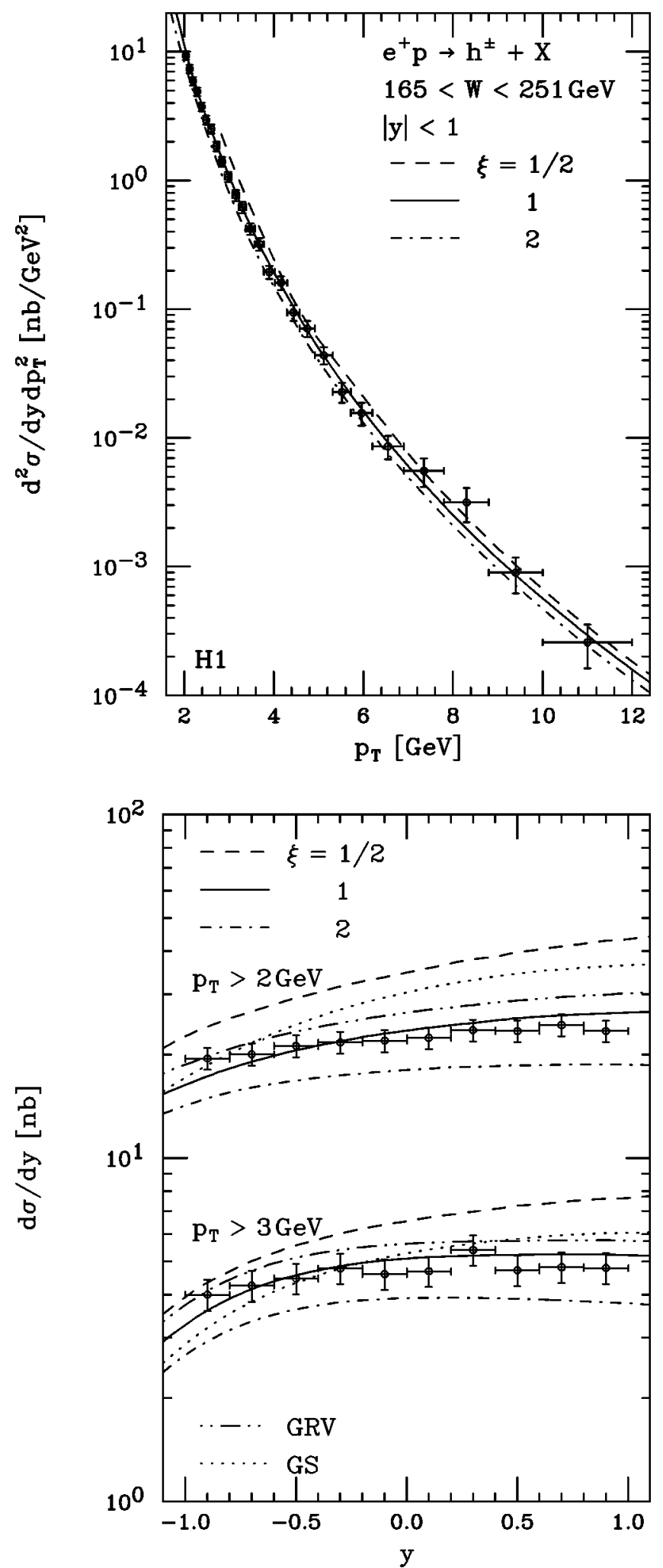

FIG. 28. Transverse momentum (top) and rapidity (bottom) dependence of the NLO inclusive charged-hadron photoproduction cross section (Kniehl, Kramer, and Pötter, 2001) compared to H1 data (Adloff et al., 1999a).

starting scale in the evolution of the heavy-quark parton densities and fragmentation functions. Next-to-leadingorder calculations in the massless scheme have been performed for photoproduction (Kniehl et al., 1995; Cac-
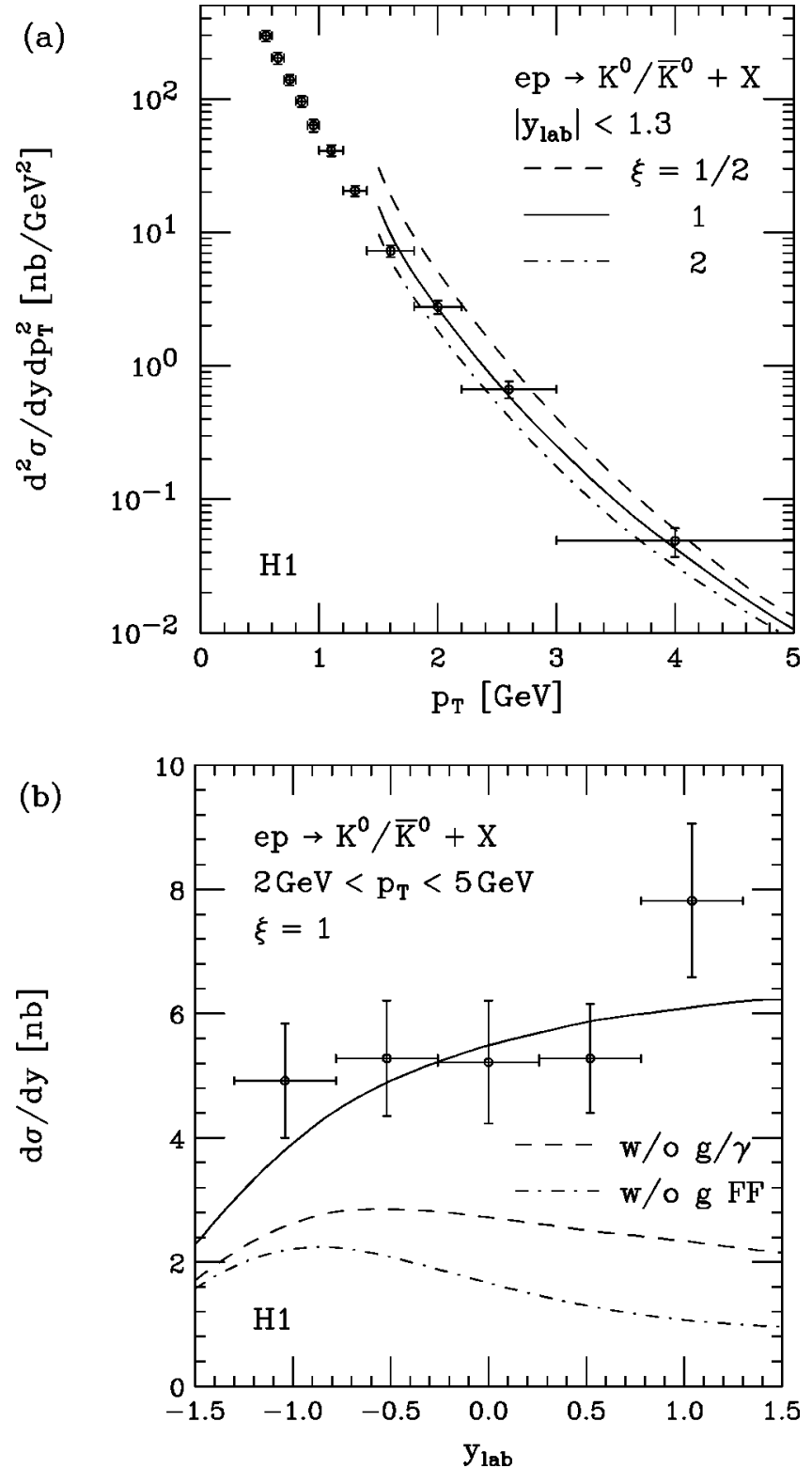

FIG. 29. Transverse momentum (top) and rapidity (bottom) dependence of the NLO inclusive neutral-kaon photoproduction cross section (Kniehl, 1997) compared to H1 data (Adloff et al., 1997).

ciari and Greco, 1996, 1997; Binnewies, Kniehl, and Kramer, 1997, 1998a, 1998b; Kniehl, Kramer, and Spira, 1997; Kramer, 1999) and photon-photon collisions (Cacciari et al., 1996; Kramer and Spiesberger, 2001) with different assumptions on the perturbative and nonperturbative components of the fragmentation function (see Sec. V.A). They have been compared with $D^{*}$ photoproduction data from H1 (Aid et al., 1996b) and ZEUS (Breitweg et al., 1997, 1999b; Derrick et al., 1997b) and photon-photon data from L3 (Acciarri et al., 1999b) and OPAL (Abbiendi et al., 2000).

If, however, the transverse momentum $p_{T}$ is of the same order as the mass of the produced hadron, the latter has to be kept in the partonic cross section 

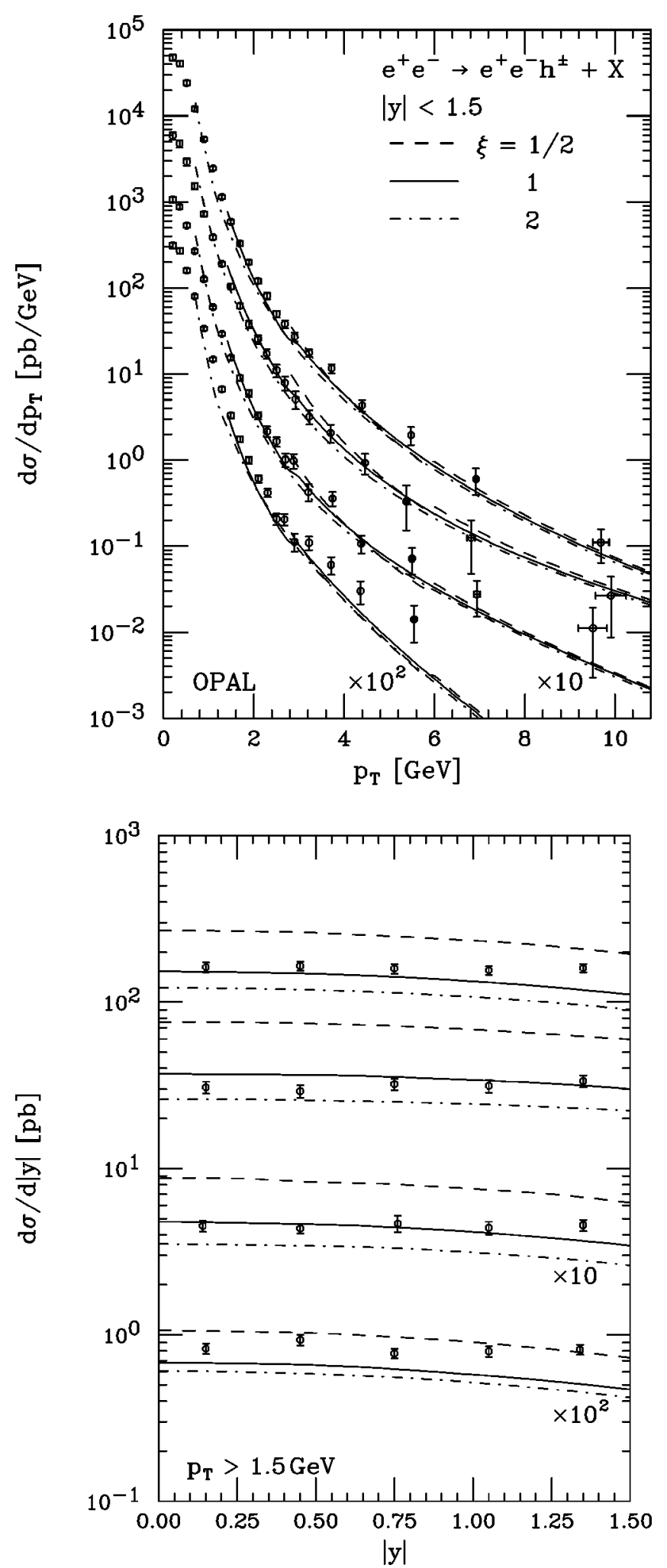

FIG. 30. Transverse momentum (top) and rapidity (bottom) dependence of the NLO inclusive charged-hadron cross section in photon-photon collisions (Kniehl, Kramer, and Pötter, 2001), integrated over $\gamma \gamma$ invariant-mass intervals $10<W$ $<30 \mathrm{GeV}, 30<W<55 \mathrm{GeV}, 55<W<125 \mathrm{GeV}$, and $10<W$ $<125 \mathrm{GeV}$ (from bottom to top in this order) and compared to OPAL data (Ackerstaff et al., 1999).

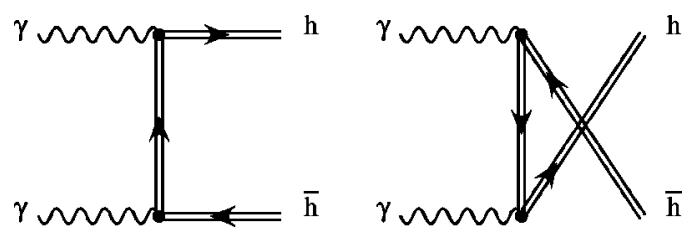

FIG. 31. Born diagrams for direct heavy-quark production.

$$
\begin{aligned}
\frac{d \sigma^{B}}{d t}= & \frac{1}{2 s} \frac{1}{\Gamma(1-\varepsilon)}\left(\frac{4 \pi s}{\left(t-p_{2}^{2}\right)\left(u-p_{2}^{2}\right)-p_{2}^{2} s}\right)^{\varepsilon} \frac{1}{8 \pi s} \\
& \times \frac{g_{a, b}^{2}}{S_{a} S_{b} C_{a} C_{b}}\left|\mathcal{M}^{B}\right|^{2} .
\end{aligned}
$$

In this case, the heavy flavors are not active in the parton densities and fragmentation functions, and only the Born diagrams in Figs. 31-33 for direct, single-, and double-resolved photon-photon scattering contribute. The corresponding matrix elements are collected in Table XII. Figures 32 and 33 and the respective matrix elements also apply to direct and resolved photoproduction. In leading-order calculations (Fritzsch and Streng, 1978) one can of course set $\varepsilon=0$, but not if NLO corrections

$$
\begin{aligned}
\frac{d \sigma^{V, F, I}}{d t}= & \frac{1}{2 s} \frac{1}{\Gamma(1-\varepsilon)}\left(\frac{4 \pi s}{\left(t-p_{2}^{2}\right)\left(u-p_{2}^{2}\right)-p_{2}^{2} s}\right)^{\varepsilon} \\
& \times \frac{1}{8 \pi s} \frac{g_{a, b}^{2}}{S_{a} S_{b} C_{a} C_{b}} \frac{\alpha_{s}}{2 \pi}\left(\frac{4 \pi \mu^{2}}{Q^{2}}\right)^{\varepsilon} \\
& \times \frac{\Gamma(1-\varepsilon)}{\Gamma(1-2 \varepsilon)}\left|\mathcal{M}^{V, F, I}\right|^{2},
\end{aligned}
$$

are included. The heavy-flavor mass has to be kept in the denominators of the virtual loop integrals and in the integrations over the solid angle [Eq. (4.12)] and invariant mass

$$
\int_{0}^{s+t-p_{1}^{2}+p_{2}^{2} s /\left(t-p_{2}^{2}\right)} d s_{i j} \frac{\left(s_{i j}-p_{1}^{2}\right)^{1-2 \epsilon}}{s_{i j}^{1-\varepsilon}}
$$

of the unresolved final-state particles. The presence of a mass usually complicates the integrals that have to be computed, but the singularity structure is generally simpler than in the massless case, since the collinear singularities are regulated by the heavy-flavor mass. The method of analytic integration was originally developed for total and single-differential cross sections of heavyflavor production in hadron collisions (Dawson, Ellis, and Nason, 1988, 1989; Beenakker et al., 1989, 1991), but

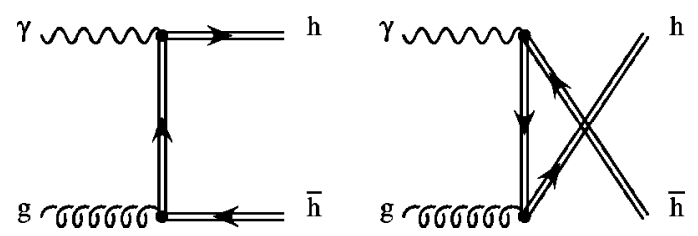

FIG. 32. Born diagrams for single-resolved heavy-quark production. 


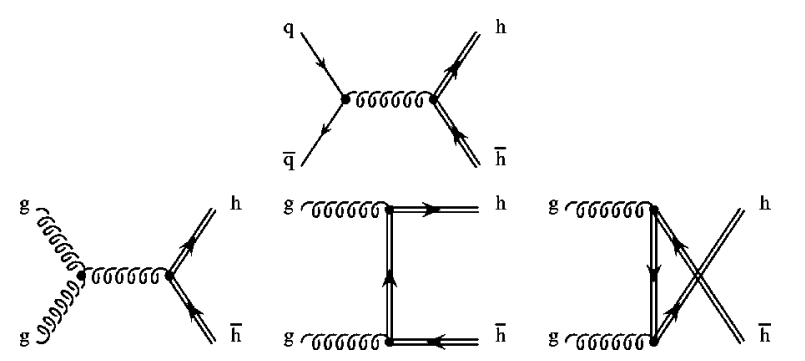

FIG. 33. Born diagrams for double-resolved heavy-quark production.

it was soon applied to photon-hadron (Ellis and Nason, 1989; Smith and van Neerven, 1992) and photon-photon collisions (Drees et al., 1993; Kühn, Mirkes, and Steegborn, 1993; Contogouris, Kamal, and Merebashvili, 1995). Later different methods based on numerical cancellations of divergences (Mangano, Nason, and Ridolfi, 1992) and phase-space slicing (Giele, Keller, and Laenen, 1996) were designed for more differential cross sections in hadron collisions. They were also subsequently extended to photon-hadron (Frixione et al., 1994a, 1994b, 1995; Frixione, Nason, and Ridolfi, 1995) and photon-photon collisions (Krämer and Laenen, 1996; Frixione, Krämer, and Laenen, 2000a, 2000b). The massless limits of the massive NLO results have recently been compared with massless NLO calculations (Cacciari, Frixione, and Nason, 2001; Kramer and Spiesberger, 2001).

After convolution with various nonperturbative fragmentation functions, the massive NLO calculations were confronted with measurements of total charm cross sections, $D$-meson $p_{T}$ and $y$ distributions, and $D \bar{D}$ azimuthal correlations by photon-hadron experiments of the Photon Emulsion (Adamovich et al., 1980, 1987), NA14/2 (Alvarez et al., 1992, 1993), E687 (Frabetti et al., 1993, 1996; Moroni et al., 1994), E691 (Anjos et al., 1989, 1990), H1 (Aid et al., 1996b), and ZEUS (Breitweg et al., 1997, 1999a, 2000e) and by the photon-photon experiments of JADE (Bartel et al., 1987), TASSO (Braun-

TABLE XII. Leading-order (LO) matrix elements squared $\left|\mathcal{M}^{B}\right|^{2}$ for $2 \rightarrow 2$ parton processes involving two, one, and no photons in $n=4-2 \varepsilon$ dimensions. $t=t-p_{2}^{2}$ and $u=u-p_{2}^{2}$ are mass-subtracted Mandelstam variables.

\begin{tabular}{ll}
\hline \hline Process & LO matrix element squared $\left|\mathcal{M}^{B}\right|^{2}$ \\
\hline$\gamma \gamma \rightarrow h \bar{h}$ & $8 N_{C}\left[\frac{t_{2}}{u_{2}}+\frac{u_{2}}{t_{2}}+\frac{4 p_{2}^{2} s}{t_{2} u_{2}}\left(1-\frac{p_{2}^{2} s}{t_{2} u_{2}}\right)\right.$ \\
& $\left.+\varepsilon\left(-1+\frac{s^{2}}{t_{2} u_{2}}\right)+\varepsilon^{2} \frac{s^{2}}{4 t_{2} u_{2}}\right]$ \\
$\gamma g \rightarrow h \bar{h}$ & $C_{F}\left|\mathcal{M}^{B}\right|_{\gamma \gamma \rightarrow h \bar{h}}^{2}(s, t, u)$ \\
$g g \rightarrow h \bar{q} \rightarrow h \bar{h}$ & $4 N_{C} C_{F}\left[\frac{t_{2}^{2}+u_{2}^{2}}{s^{2}}+\frac{2 p_{2}^{2}}{s}+\frac{\varepsilon}{2}\right]$ \\
\hline \hline
\end{tabular}

schweig et al., 1990), TPC/2 $\gamma$ (Alston-Garnjost et al., 1990), AMY (Takashimizu et al., 1996), TOPAZ (Enomoto et al., 1994a, 1994b), ALEPH (Buskulic et al., 1995), L3 (Acciarri et al., 1999a, 2001a, 2001b), and OPAL (Abbiendi et al., 2000, 2001a).

The massive NLO total charm cross section (Frixione et al., 1994a) shown in Fig. 34 (top) describes the old fixed-target and the new HERA photoproduction data well within the scale uncertainty (Aid et al., 1996b). The H1 measurement (Adloff et al., 1999b) of the total bottom cross section in Fig. 34 (bottom) is, however, severely underestimated by the massive NLO calculation, as are the corresponding ZEUS measurement (Breitweg et al., 2001) and the hadroproduction measurements from CDF (Abe et al., 1993, 1995) and D0 (Abbott et al., 2000a, 2000c). It should be noted that the NLO predictions are rather sensitive to the photon (Glück, Reya,
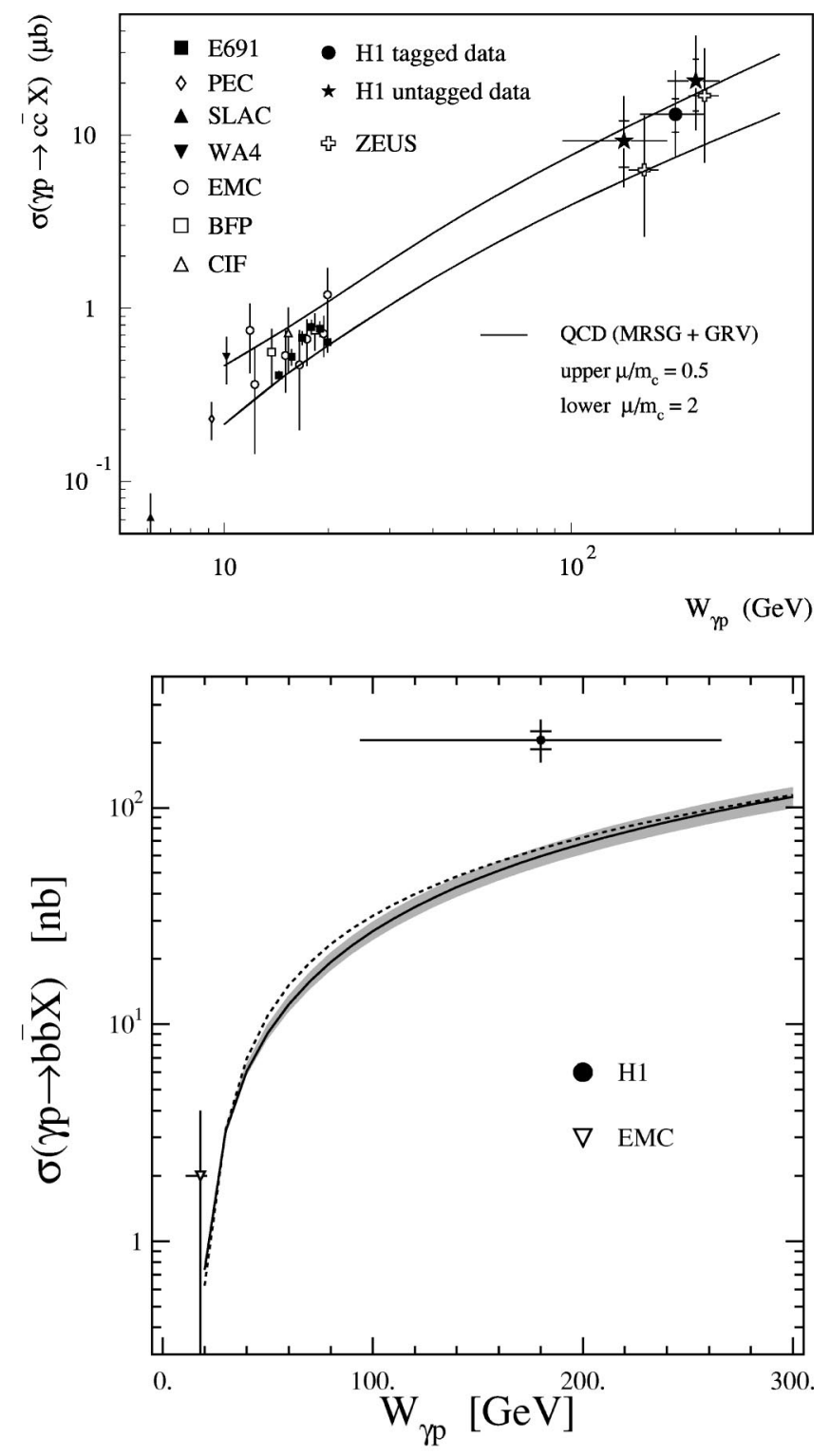

FIG. 34. Photon-proton center-of-mass energy dependence of the massive NLO total charm (top) and bottom (bottom) cross sections (Frixione et al., 1994a) compared to fixed-target and HERA data (Aid et al., 1996b; Adloff et al., 1999b). 
and Vogt, 1992b) and proton (Martin, Roberts, and Stirling, 1993) parton densities, and also to a lesser degree to the fragmentation model and the masses of the charm $\left(m_{c}=1.5 \mathrm{GeV}\right)$ and bottom quarks $\left(m_{b}\right.$ $=4.75 \mathrm{GeV}$ ), but these variations cannot account for the discrepancy in the bottom cross section (Blair et al., 2000). NLO massless calculations (Binnewies, Kniehl, and Kramer, 1997; Cacciari and Greco, 1997) cannot be applied to total cross sections due to the absence of a hard scale. They can, however, be applied to $D^{*}$ transverse momentum (top) and pseudorapidity (bottom) distributions as shown in Fig. 35. Massless distributions are usually larger than their massive counterparts, and they describe the experimental data better (Breitweg et al., 1999a), particularly in the large- $p_{T}$ region, where the massive calculation fits the data only if one chooses low values for $m_{c}=1.2 \mathrm{GeV}$, the renormalization scale $\mu=0.5 m_{T}$, and the Peterson fragmentation function parameter $\varepsilon=0.02$. The shapes of the measured pseudorapidity distributions are not well described by either the massive or the massless calculations. This fact has attracted considerable theoretical effort, from the consideration of "drag effects" due to the beam remnants (Norrbin and Sjöstrand, 2000) to $c \bar{c}$ vector-meson components of the photon wave function (Berezhnoy and Likhoded, 2001) and heavy-light quark recombination models inspired by heavy quarkonia (Berezhnoy, Kiselev, and Likhoded, 2000; Braaten, Jia, and Mehen, 2002), which may also have relevance for asymmetries in $B$ physics. The cut on $p_{T}>2 \mathrm{GeV}$ is probably too close to $m_{c}$ for the massless calculations to be reliable. In the remaining $p_{T}$ regions, different charm densities in the photon allow for a somewhat improved description of the data (Breitweg et al., 1999a).

The measured total charm cross sections in photonphoton collisions in Fig. 36 (top) are again well described by massive NLO calculations (Drees et al., 1993), whereas the total bottom cross sections (bottom) are significantly underestimated. Even though the experimental and theoretical uncertainties are quite large, they cannot account for the discrepancy in the bottom case. As for the $D^{*}$ transverse momentum (top) and pseudorapidity (bottom) distributions shown in Fig. 37, they are well described by the massless calculation, despite the low transverse momenta studied $\left(p_{T}\right.$ $>2 \mathrm{GeV})$. Surprisingly, the massive calculation agrees with the data for larger $p_{T}\left(p_{T}>3 \mathrm{GeV}\right)$ but not for small $p_{T}$, where it should be more appropriate. Even low values of $m_{c}$ and $\mu$ cannot bring the massive calculation into agreement with the measured pseudorapidity distribution.

With future $e^{+} e^{-}$and $e p$ colliders, it will be possible not only to extend the kinematic ranges and production rates of charm and bottom quarks (Krämer and Laenen, 1996; Jankowski, Krawczyk, and Wing, 2001), but also to study the heavier top quark and its properties (Kühn, Mirkes, and Steegborn, 1993; Bernreuther, Ma, and McKellar, 1995; Choi and Hagiwara, 1995).

\section{Quarkonia}

As was briefly discussed in Sec. V.A, the factorization formalism of nonrelativistic QCD (Bodwin, Braaten, and Lepage, 1995) provides a rigorous theoretical framework for the description of heavy-quarkonium production and decay. This formalism implies a separation of short-distance coefficients, which can be calculated perturbatively as expansions in the strong-coupling constant $\alpha_{s}$, from long-distance operator matrix elements, which must be extracted from experiment. The relative importance of the latter can be estimated by means of velocity scaling rules (Lepage et al., 1992), i.e., they are predicted to scale with a definite power of the heavy-quark velocity $v$ in the limit $v \ll 1$. In this way, the theoretical predictions are organized as double expansions in $\alpha_{s}$ and $v$. A crucial feature of this formalism is that it takes into account the complete structure of the $h \bar{h}$ Fock space, which is spanned by the states $n$ $=\left[\underline{a},{ }^{2 S+1} L_{J}\right]$ with definite spin $S$, orbital angular momentum $L$, total angular momentum $J$, and color multiplicity $\underline{a}=\underline{1}, \underline{8}$. In particular, nonrelativistic QCD predicts the existence of color-octet processes in nature. This means that $h \bar{h}$ pairs are produced at short distances in color-octet states and subsequently evolve into physical (color-singlet) quarkonia by the nonperturbative emission of soft gluons. In the limit $v \rightarrow 0$, the traditional color-singlet model (Baier and Rückl, 1981; Berger and Jones, 1981;) is recovered. The greatest triumph of nonrelativistic QCD was that it was able to describe correctly the cross section of inclusive charmonium hadroproduction measured in $p \bar{p}$ collisions at the Fermilab Tevatron (Braaten and Fleming, 1995; Cho and Leibovich, 1996a, 1996b), which had turned out to be more than one order of magnitude in excess of the theoretical prediction based on the color-singlet model (Abe et al., 1992, 1997). An alternative model, which also predicts the existence of color-octet processes, is the duality or color-evaporation model, in which conservation of the color quantum number is ignored in the hard interaction and assumed to be recovered only through the exchange of nonperturbative soft gluons with the underlying event (Fritzsch, 1977; Halzen, 1977; Glück, Owens, and Reya, 1978). The short-distance color-singlet and color-octet cross sections are related to those of open heavy-quark production by statistical factors and to those of the physically observed mesons by constant universal factors. While this model can also account for the Tevatron data (Amundson et al., 1997), it lacks the status of nonrelativistic QCD as an effective field theory. Similar objections may be raised to a model that allows the spin flip and color flip of the heavy-quark pair through a perturbative gluon exchange with an isotropic classical color field generated by bremsstrahlung of the initial-state partons (Hoyer and Peigné, 1999; Hoyer, Marchal, and Peigné, 2000). This "hard comover scattering" model can explain not only the observed ratio of $\chi_{c, 1}$ and $\chi_{c, 2}$ hadroproduction cross sections, but also the observed nonpolarization of hadroproduced $J / \psi$ and $\psi^{\prime}$ mesons. In both of these cases the color-singlet model and non- 


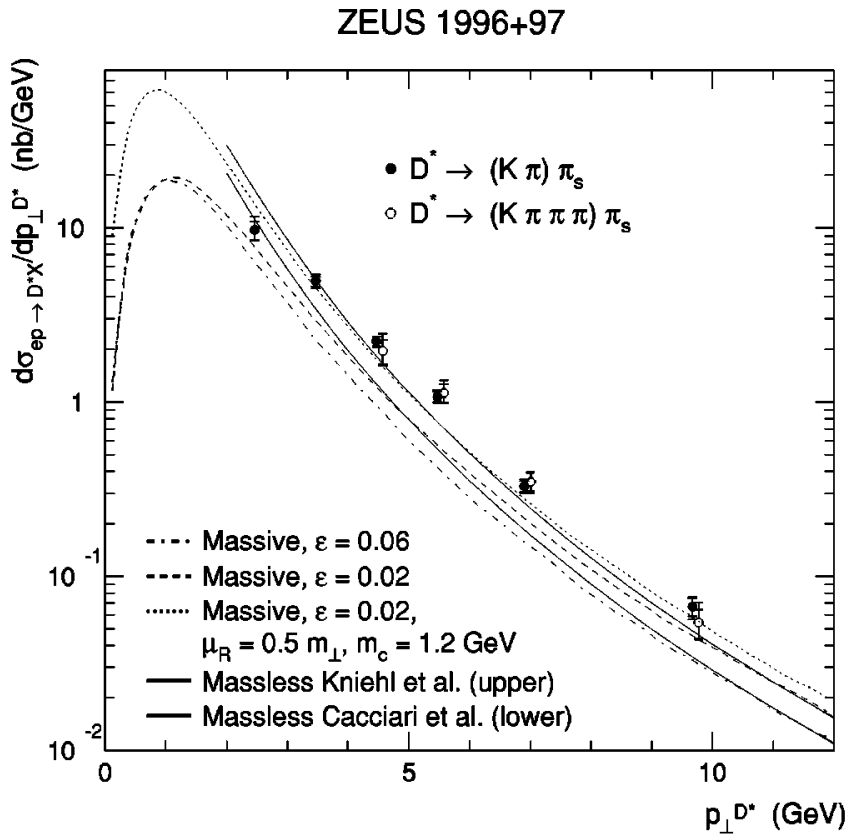

ZEUS 1996+97
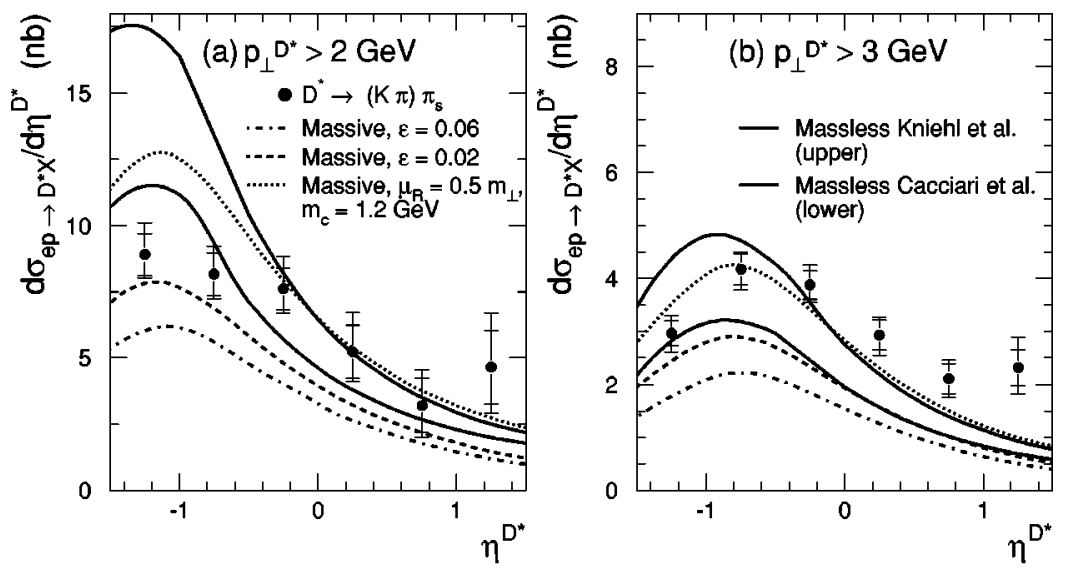

FIG. 35. Transverse momentum (top) and pseudorapidity (bottom) dependence of the massive (Frixione et al., 1994a) and two different massless (Binnewies, Kniehl, and Kramer, 1997; Cacciari and Greco, 1997) NLO $D^{*}$ photoproduction cross sections compared to ZEUS data (Breitweg et al., 1999a).
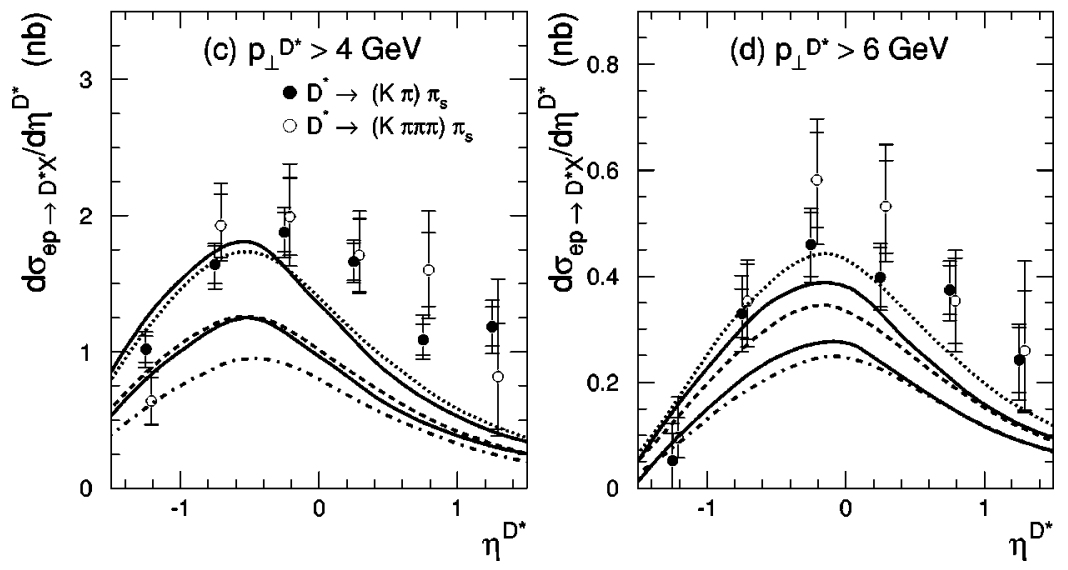

relativistic QCD have some difficulty in explaining the data. Quarkonium production in $p \bar{p}$ and $e p$ collisions has recently been reviewed by Krämer (2001).

In order to convincingly establish the phenomenological significance of the color-octet processes, it is indispensable to identify them in other kinds of high-energy experiments as well. In photon-photon and photon- hadron collisions, quarkonia can be produced by direct, single-resolved, and double-resolved processes. Which of the various photon-, quark-, and gluon-initiated processes contribute to the production of a given quarkonium state depends on the leading operator matrix elements in the nonrelativistic QCD velocity expansion and on the additional particles in the final state. In inelastic 

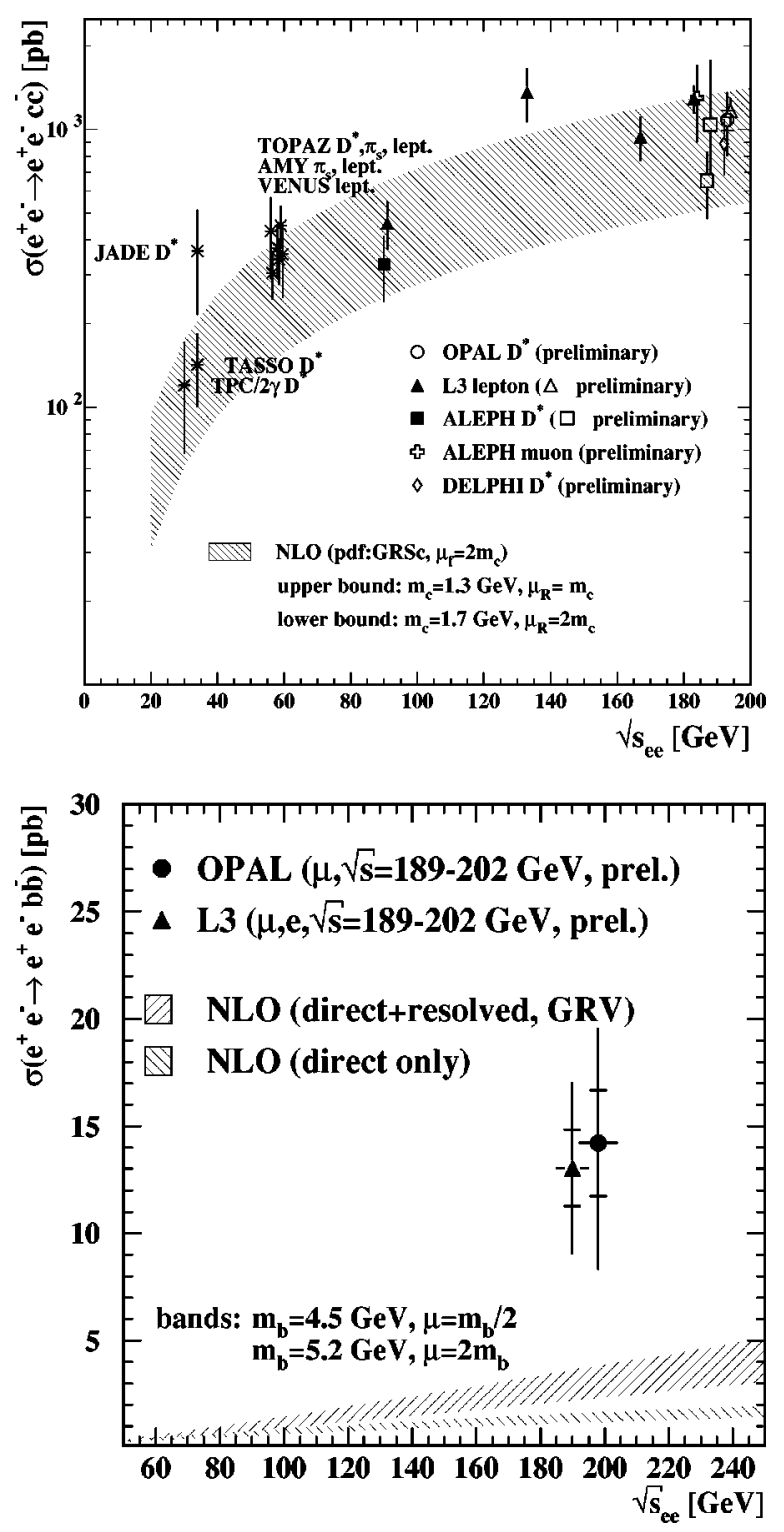

FIG. 36. $e^{+} e^{-}$center-of-mass energy dependence of the massive NLO total charm (top) and bottom (bottom) cross sections in photon-photon collisions (Drees et al., 1993) compared to TPC/ $2 \gamma$, PETRA, TRISTAN, and LEP data (Abbiendi et al., 2001a, 2001b).

processes, the quarkonium state is produced with nonzero transverse energy and a balancing jet or photon. In this case the inclusive hadronic production cross section is given by Eq. (5.14) with $D_{H / c}\left(z, M_{c}^{2}\right)=\delta(1-z)$. This means that $p_{T}$ and $y$ now relate to the transverse momentum and rapidity of the observed quarkonium state, and the partonic cross section is given by Eq. (5.16), where the mass $p_{2}^{2}$ is identified with the quarkonium mass $M^{2}$ (=4 $m_{h}^{2}$ in the static limit).

Since its discovery in 1974 , the $J / \Psi$ charmonium particle has remained at the center of interest due to its relatively large production cross section and easy experimental identification in the leptonic decay modes. The diagrams contributing to the production of its leading color-singlet state $\left[1,{ }^{3} S_{1}\right]$ are shown in Figs. 38-40. In photon-photon scattering, they relate to direct, single-
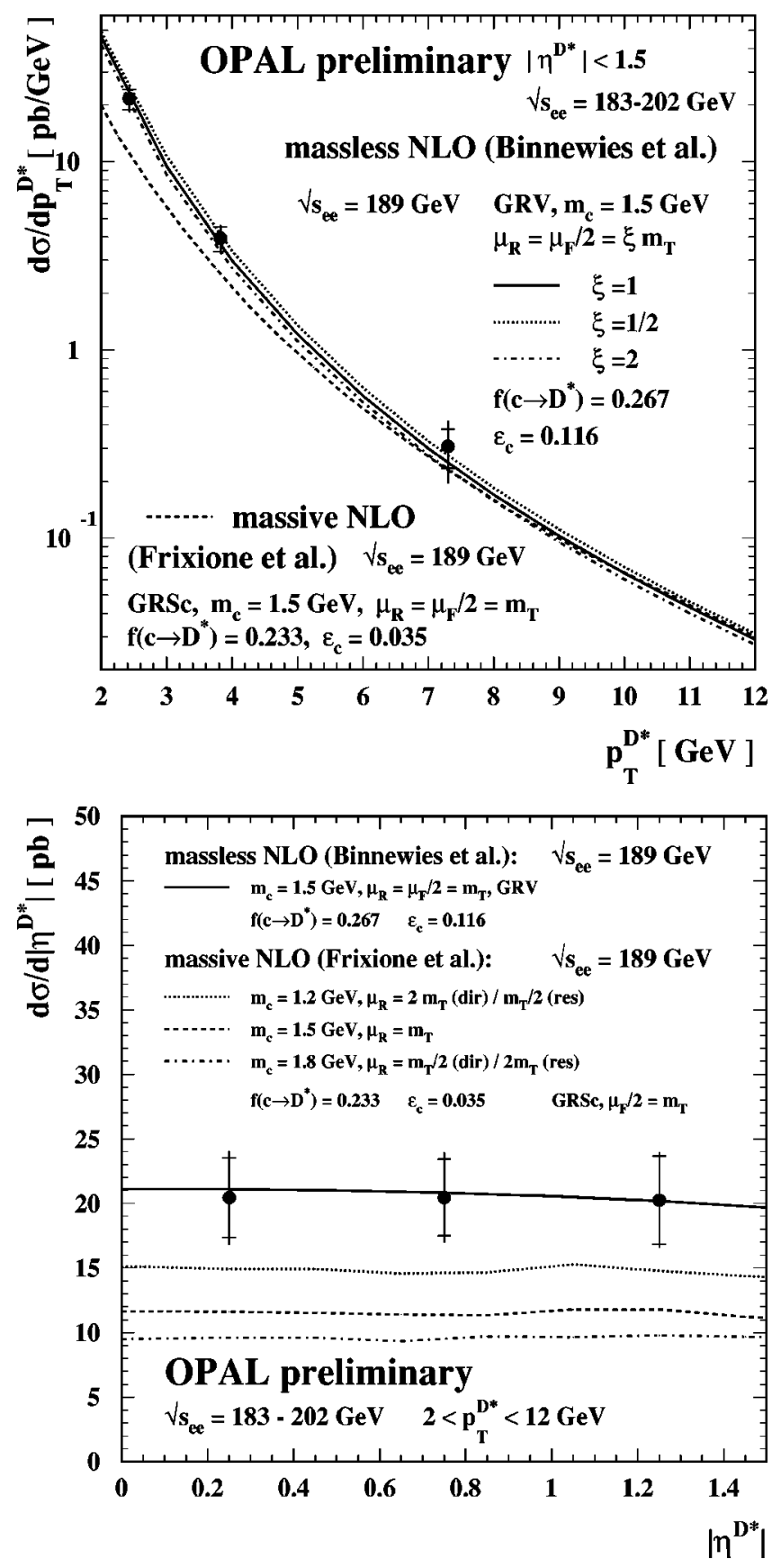

FIG. 37. Transverse momentum (top) and pseudorapidity (bottom) dependence of the massive (Frixione, Krämer, and Laenen, 2000a) and a massless (Binnewies, Kniehl, and Kramer, 1996b, 1998a) next-to-leading-order $D^{*}$ photonphoton cross section compared to OPAL data (Abbiendi et al., 2001a).

resolved, and double-resolved processes. In photoproduction, the last two apply to direct and resolved processes, while in hadroproduction only the diagrams in Fig. 40 contribute. The corresponding hard scattering matrix elements are related by simple transformations of couplings and color factors and are listed in Table XIII. The diagrams in Figs. 38-40 also contribute to the production of one of the leading color-octet states, $\left[\underline{8},{ }^{3} S_{1}\right]$, when the final photon in Fig. 38 is replaced with a gluon and the color factors are changed appropriately. At the 


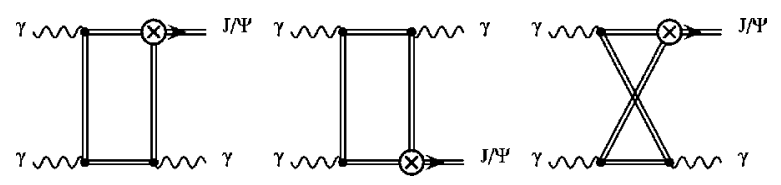

FIG. 38. Born diagrams for direct color-singlet $J / \Psi+\gamma$ production. The charm quark lines in the loop run in both directions.

same order $v^{4}$ relative to $\left\langle O_{1}^{J / \Psi}\left({ }^{3} S_{1}\right)\right\rangle$, the operator matrix elements $\left\langle O_{8}^{J / \Psi}\left({ }^{1} S_{0}\right)\right\rangle$ and $\left\langle O_{8}^{J / \Psi}\left({ }^{3} P_{J}\right)\right\rangle$ with $J$ $=0,1,2$ contribute in photon- and gluon-quark scattering with final-state quarks and in quark-antiquark annihilation with final-state gluons (Japaridze and Tkabladze, 1998; Ma, McKellar, and Paranavitane, 1998; Klasen, Kniehl, Mihaila, and Steinhauser, 2001a; Godbole, Indumathi, and Krämer, 2002).

In the photon and gluon fusion processes described above, the two internal quark propagators are off shell by $p_{T}^{2}$, so that the differential cross section falls like $1 / p_{T}^{8}$. However, when $p_{T} \gg M$, the quarkonium mass $M$ can be considered small and the cross section should scale like any other single-particle inclusive cross section as $1 / p_{T}^{4}$. The dominant quarkonium production mechanism at sufficiently large $p_{T}$ must therefore be via fragmentation of a final-state parton (see Sec. V.A) in the partonic processes shown in Figs. 7-9 (Braaten and Yuan, 1993).

In Fig. 41, the leading-order color-singlet and the sum of color-singlet and color-octet contributions are compared with preliminary data on $J / \Psi$ production in photon-photon collisions from DELPHI (TodorovaNova, 2001; Chapkin, 2002). The agreement of the nonrelativistic QCD prediction with the data is excellent, although the statistical and theoretical uncertainties from the renormalization and factorization scales, from $m_{c}=1.5 \mathrm{GeV}$, and the numerical values of the nonrelativistic QCD operator matrix elements (Braaten, Kniehl, and Lee, 2000) are still large (Klasen, Kniehl, Mihaila, and Steinhauser, 2002). Single-resolved processes are far more important than double-resolved and direct processes (see also Godbole, Indumathi, and Krämer, 2002). The total color-singlet contribution is insufficient to describe the data, and the size of the cross section is sensitive to the color-octet operator values extracted at the Tevatron. The shapes of color-singlet and color-octet contributions can, however, be distinguished only at larger $p_{T}$

At the scale of the charm mass, the strong coupling $\alpha_{s}$ is still relatively large $(\simeq 0.3)$ and NLO corrections can become important. The real corrections to $J / \Psi$ produc-

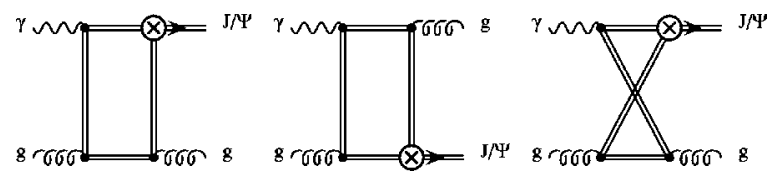

FIG. 39. Born diagrams for single-resolved color-singlet $J / \Psi$ + jet production.

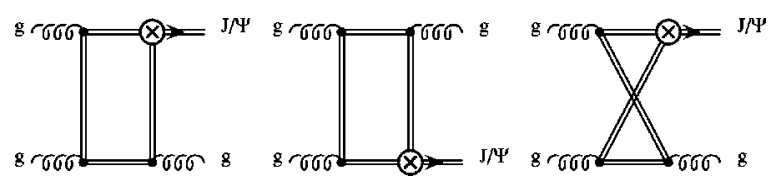

FIG. 40. Born diagrams for double-resolved color-singlet $J / \Psi+$ jet production.

tion in direct photon-photon collisions, which become dominant at large $p_{T}$, have recently been calculated with an invariant-mass cutoff for the associated dijet system. Figure 42 shows that the leading-order cross section is enhanced by about an order of magnitude at large $p_{T}$, where the missing virtual corrections are no longer important and the uncertainty from the exact value of the cutoff is reduced (Klasen, Kniehl, Mihaila, and Steinhauser, 2001). In a complete NLO calculation, there would of course be no dependence on the cutoff.

A full NLO calculation is available for inelastic colorsinglet $J / \Psi$ production in direct photon-hadron collisions (Krämer et al., 1995; Krämer, 1996). The direct process dominates at the relatively large values of $z(z$ $\left.=p_{P} \cdot p_{J / \Psi} / p_{P} \cdot p_{\gamma} \simeq E_{J / \Psi} / E_{\gamma} \geqslant 0.3\right)$ currently observed at HERA. Recent H1 data (Krüger, 2000) also show evidence for resolved photoproduction, which is closely connected to hadroproduction and contributes mostly at low $z \leqslant 0.3$. This region is dominated by color-octet processes in nonrelativistic QCD (Beneke, Krämer, and Vänttinen, 1998; Kniehl and Kramer, 1999), but also in the color-evaporation model (Éboli, Gregores, and Halzen, 1999). As can be seen in Fig. 43, the direct leading-order color-singlet prediction can almost account for the data. Within the relatively large uncertainty of the theoretical parameters (shaded band), the direct color-octet processes improve the agreement at intermediate $z$, but show a stronger rise than the data at large $z$ (Cacciari and Krämer, 1996; Ko, Lee, and Song, 1996; Amundson, Fleming, and Maksymyk, 1997). However, this region is close to the kinematic limit and is influenced by the nonperturbative soft transition of the color-octet state into the physical $J / \Psi$ meson, so that leading-order predictions are not reliable. NLO corrections for color-octet photoproduction have unfortunately been calculated only for the total cross section (Maltoni, Mangano, and Petrelli, 1998), but higher-order terms have been resummed using nonrelativistic shape functions (Beneke, Rothstein, and Wise, 1997; Beneke, Schuler, and Wolf, 2000). Relativistic corrections to the color-singlet state have been evaluated, but there is unfortunately no agreement on their effects in the literature (Greub et al., 1993; Khan and Hoodboy, 1996; Ma, McKellar, and Paranavitane, 2000). Higher-twist effects due to multiple interactions with the proton or photon remnants should be suppressed as a power of $\Lambda^{2} /\left(Q^{2}\right.$ $+p_{T}^{2}$ ) with $Q=m_{c}, m_{c} v$, or $m_{c} v^{2}$ (Beneke, Krämer, and Vänttinen, 1998) and not as a power of $\Lambda^{2} /\left(4 m_{c}^{2}\right)$ (Ma, 1997), since only the first result leads to the intuitively expected $\Lambda^{2} / p_{T}^{2}$ behavior at large $p_{T}$. However, the discrepancy between the nonrelativistic QCD prediction and the HERA data can clearly be reduced if higher- 
TABLE XIII. Leading-order (LO) matrix elements squared $\left|\mathcal{M}^{B}\right|^{2}$ for direct, single-resolved, and double-resolved color-singlet $J / \Psi$ production.

\begin{tabular}{ll}
\hline \hline Process & LO matrix element squared $\left|\mathcal{M}^{B}\right|^{2}$ \\
\hline$\gamma \gamma \rightarrow\left\langle O_{1}^{J / \Psi}\left({ }^{3} S_{1}\right)\right\rangle \gamma$ & $\frac{3072\left\langle O_{1}^{J / \Psi}\left({ }^{3} S_{1}\right)\right\rangle m_{c}}{3-2 \varepsilon} \cdot \frac{(2-5 \varepsilon) s t u(s+t+u)+2(1-\varepsilon)^{2}\left(s^{2} t^{2}+s^{2} u^{2}+t^{2} u^{2}\right)}{(s+t)^{2}(s+u)^{2}(t+u)^{2}}$ \\
$\gamma g \rightarrow\left\langle O_{1}^{J / \Psi}\left({ }^{3} S_{1}\right)\right\rangle g$ & $\frac{C_{F}}{2 N_{C}}\left|\mathcal{M}^{B}\right|_{\gamma \gamma \rightarrow\left\langle O_{1}^{J / \Psi}\left({ }^{3} S_{1}\right)\right\rangle \gamma}^{2}$ \\
$g g \rightarrow\left\langle O_{1}^{J / \Psi}\left({ }^{3} S_{1}\right)\right\rangle g$ & $\frac{N_{C}^{2}-4}{4 N_{C}}\left|\mathcal{M}^{B}\right|_{\gamma g \rightarrow\left\langle O_{1}^{J / \Psi}\left({ }^{3} S_{1}\right)\right\rangle g}^{2}$ \\
\hline \hline
\end{tabular}

order effects of multigluon emission are approximately taken into account in the extraction of the operator matrix elements at the Tevatron (Kniehl and Kramer, 1999).

While the shape of the $J / \Psi$ energy distribution in Fig. 43 is already well described by the leading-order colorsinglet calculation, the shape of the leading-order transverse momentum distribution in Fig. 44, which falls like $\alpha_{s}^{2} m_{c}^{4} / p_{T}^{8}$, differs markedly from the HERA data. The data clearly require the NLO color-singlet contribution, which is dominated by $t$-channel gluon exchange and falls only like $\alpha_{s}^{3} m_{c}^{2} / p_{T}^{6}$ (Krämer et al., 1995; Krämer, 1996). A similar scaling is observed by contributions from charm quark fragmentation in direct photoproduction, which exist at $\mathcal{O}\left(\alpha_{s}^{3}\right)$. They are suppressed at low $p_{T}$, but exceed the color-singlet production via photongluon fusion at $p_{T} \geqslant 10 \mathrm{GeV}$ according to leading-order (Godbole, Roy, and Sridhar, 1996) and NLO calculations (Kniehl and Kramer, 1997a, 1997b). Gluon fragmentation in resolved photoproduction exceeds the fu-

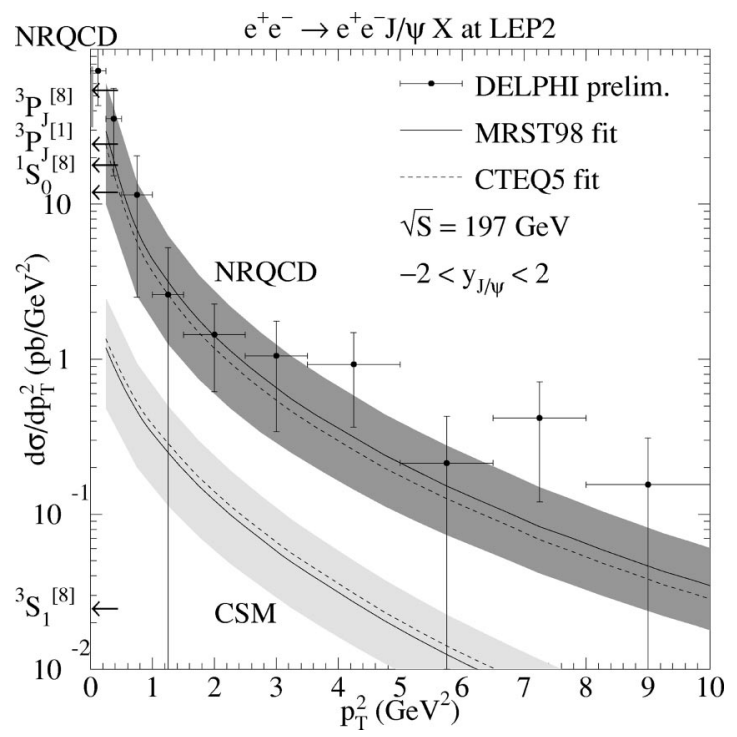

FIG. 41. Leading-order squared transverse momentum distribution of $J / \Psi$ mesons produced in photon-photon collisions at LEP2. The total nonrelativistic QCD result describes the preliminary data from DELPHI (Todorova-Nova, 2001; Chapkin, 2002) well, while the color-singlet model (CSM) is an order of magnitude too low. sion process at $p_{T} \geqslant 5 \mathrm{GeV}$ due to a large enhancement by NLO corrections (Kniehl and Kramer, 1997a, 1997b).

Further information about the underlying production mechanism (color-singlet model, color-evaporation model, or nonrelativistic QCD) may be gained from the energy spectrum of $J / \Psi$ 's produced in polarized photonhadron collisions (Chao et al., 2000; Japaridze, Nowak, and Tkabladze, 2000) and of $J / \Psi$ 's produced in association with a photon, where the color-octet contribution dominates at intermediate $z$ (Kim and Reya, 1993; Cacciari, Greco, and Krämer, 1997; Mehen, 1997). Another very sensitive observable is the energy spectrum of the polar angle in polarized $J / \Psi$ decays (Beneke, Krämer, and Vänttinen, 1998). At the upgraded HERA collider one may furthermore hope to observe and study the photoproduction of other quarkonia like the $S$-wave states $\psi^{\prime}$ (Morii, Roy, and Sudoh, 2000; Brugnera, 2001) and $\eta_{c}$ (Chao, Hao, and Yuan, 1999) or the $P$-wave states $\chi_{c}(\mathrm{Ma}, 1996 \mathrm{~b})$ and $h_{c}$ (Fleming and Mehen, 1998). Bottomonia have a smaller relative squared velocity $v^{2} \simeq 0.1$ than charmonia with $v^{2} \simeq 0.3$ (Quigg and Rosner, 1979) and should therefore be better described by the nonrelativistic QCD velocity expansion. Unfortunately, the photoproduction cross section for $Y$ is more than two orders of magnitude smaller than the one for $J / \Psi$ due to the smaller electric charge and larger mass of the bottom quark.

The large conceptual and numerical uncertainties in quarkonium physics clearly call for an increased theoretical effort, in particular for a full NLO calculation of color-singlet production at hadron colliders and of coloroctet production at photon and hadron colliders. In open flavor production, the NLO corrections should be calculated for the photoproduction of two light or heavy hadrons and of hadrons in association with jets, which would help to disentangle the flavor (e.g., charm) content of photons and protons and to resolve the question of why massive calculations cannot describe the observed bottom cross sections.

\section{PROMPT-PHOTON PRODUCTION}

The many facets that photons exhibit in the initial state of hard scattering processes can be rediscovered in 
ee $\rightarrow \mathrm{J} / \psi+\mathrm{X}$ at LEP2 (Bremsstr.)

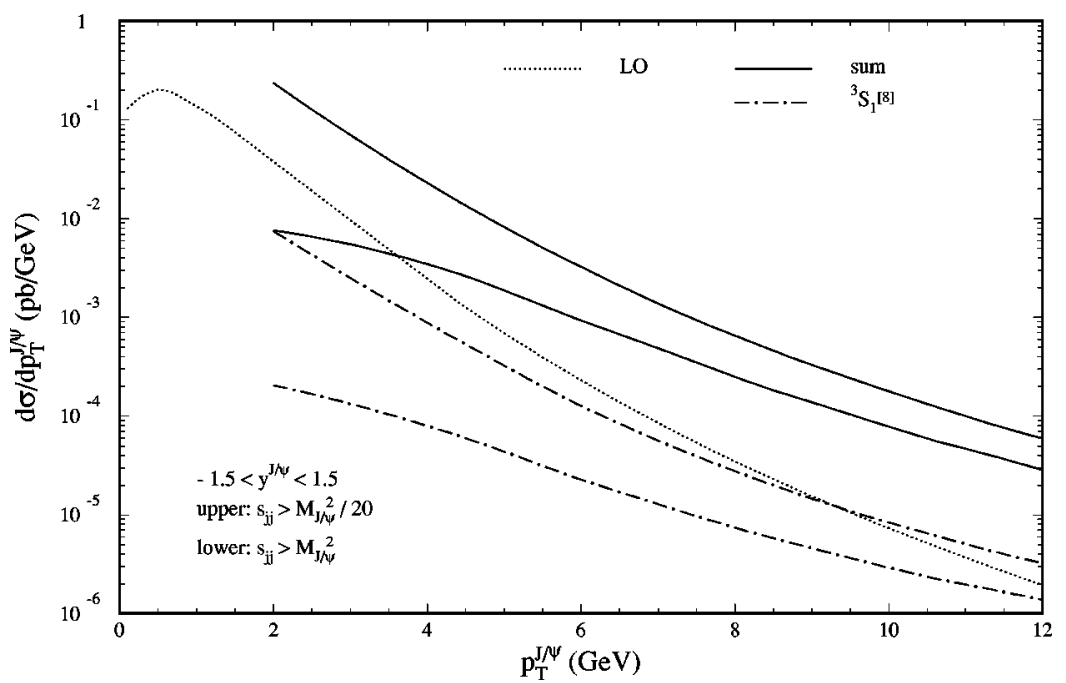

FIG. 42. Transverse momentum spectrum for $J / \Psi$ production in direct photon-photon collisions at LEP2. At large $p_{T}$, the NLO real emission corrections enhance the leadingorder (LO) cross section by an order of magnitude. From Klasen, Kniehl, et al. 2001.

their production in the final state: If produced inclusively, prompt photons are related to hadrons by important fragmentation contributions; if produced in isolation, they exhibit more perturbative aspects due to their pointlike coupling to quarks; if produced in association with jets, they become powerful tools for constraining the initial parton densities. All of these issues will be discussed in this section.

\section{A. Fragmentation}

Since photons couple to charged quarks, they can be produced directly in hard scattering interactions. If the photon is radiated from a massless final-state quark, it exhibits a collinear $1 / \varepsilon$ singularity proportional to the $P_{\gamma \leftarrow q}(x)$ splitting function and the underlying leading-

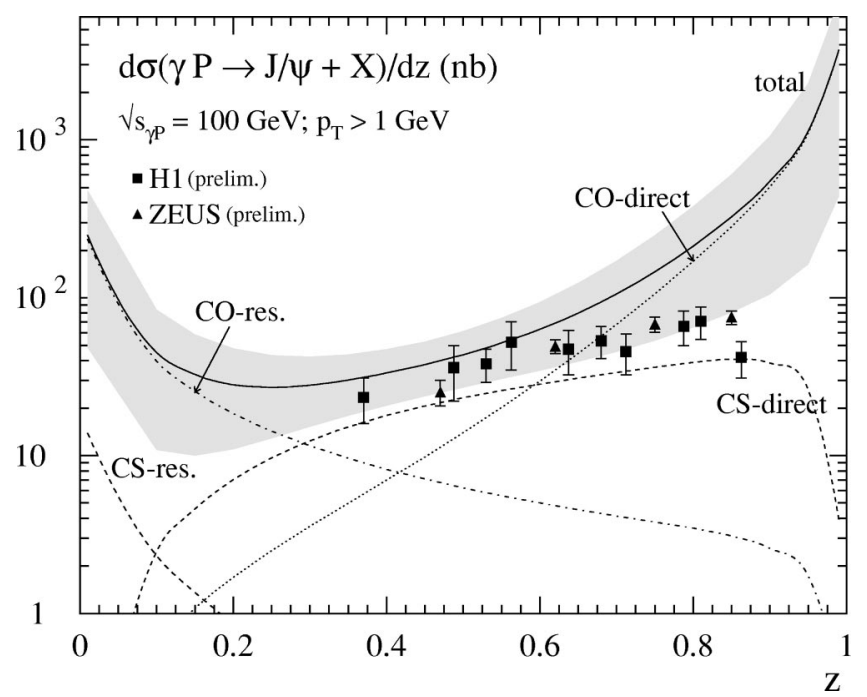

FIG. 43. Direct and resolved contributions to the color-singlet and color-octet $J / \Psi$ energy distribution at HERA. From Krämer, 2001. order matrix element. This $1 / \varepsilon$ pole has to be absorbed into a nonperturbative fragmentation function, just as the collinear singularity of an initial-state photon had to be absorbed into a nonperturbative parton density. The photon fragmentation functions $D_{\gamma / i}$ are thus intimately related to the parton densities in the photon $f_{i / \gamma}$.

The evolution equations of these fragmentation functions are of a form similar to Eqs. (3.5) with the role of initial and final particles reversed. They are given by (Koller, Walsh, and Zerwas, 1979)

$$
\begin{aligned}
\frac{d D_{\gamma / q}\left(Q^{2}\right)}{d \ln Q^{2}}= & \frac{\alpha}{2 \pi} P_{\gamma \leftarrow q} \otimes D_{\gamma / \gamma}\left(Q^{2}\right)+\frac{\alpha_{s}\left(Q^{2}\right)}{2 \pi} \\
& \times\left[P_{q \leftarrow q} \otimes D_{\gamma / q}\left(Q^{2}\right)+P_{g \leftarrow q}\right. \\
& \left.\otimes D_{\gamma / g}\left(Q^{2}\right)\right],
\end{aligned}
$$

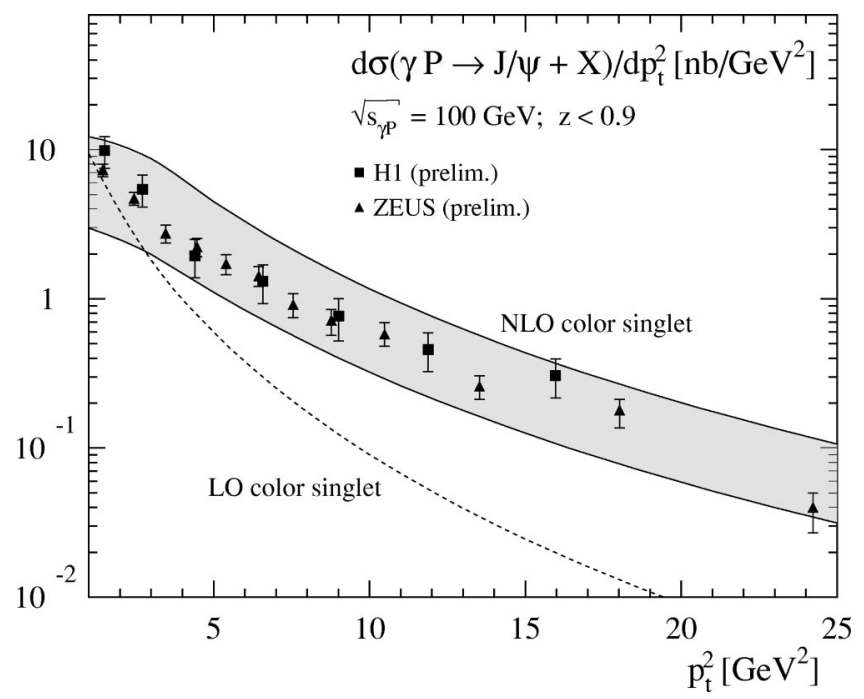

FIG. 44. $J / \Psi$ transverse momentum distribution in leadingorder (LO) and next-to-leading-order (NLO) direct colorsinglet photoproduction. From Krämer, 2001. 


$$
\begin{aligned}
\frac{d D_{\gamma / g}\left(Q^{2}\right)}{d \ln Q^{2}}= & \frac{\alpha}{2 \pi} P_{\gamma \leftarrow g} \otimes D_{\gamma / \gamma}\left(Q^{2}\right)+\frac{\alpha_{s}\left(Q^{2}\right)}{2 \pi} \\
& \times\left[P_{q \leftarrow g} \otimes D_{\gamma / q}\left(Q^{2}\right)+P_{g \leftarrow g}\right. \\
& \left.\otimes D_{\gamma / g}\left(Q^{2}\right)\right], \\
\frac{d D_{\gamma / \gamma}\left(Q^{2}\right)}{d \ln Q^{2}}= & \frac{\alpha}{2 \pi} P_{\gamma \leftarrow \gamma} \otimes D_{\gamma / \gamma}\left(Q^{2}\right)+\frac{\alpha}{2 \pi} \\
& \times\left[P_{q \leftarrow \gamma} \otimes D_{\gamma / q}\left(Q^{2}\right)+P_{g \leftarrow \gamma}\right. \\
& \left.\otimes D_{\gamma / g}\left(Q^{2}\right)\right]
\end{aligned}
$$

for a single quark flavor $q$. The timelike splitting functions $P_{j \leftarrow i}$ are identical to the spacelike splitting functions in leading order (Altarelli and Parisi, 1977), but differ in next-to-leading order (Curci, Furmanski, and Petronzio, 1980; Furmanski and Petronzio, 1980; Floratos, Kounnas, and Lacaze, 1981). Like the evolution equations (3.5) for photon densities, Eqs. (6.1) contain inhomogeneous terms: The quark-photon splitting function can be obtained in leading order from $P_{g \leftarrow q}$ by the transformation $P_{\gamma \leftarrow q}=e_{q}^{2} / C_{F} P_{g \leftarrow q}$. The gluon-photon splitting function enters only in next-to-leading order and is given by (Aurenche, Chiappetta, et al., 1993)

$$
\begin{aligned}
P_{\gamma \leftarrow g}(x)= & \frac{\alpha_{s}\left(Q^{2}\right)}{2 \pi} \frac{e_{q}^{2} T_{R}}{2}\left[-4+12 x-\frac{164}{9} x^{2}+\frac{92}{9 x}\right. \\
& +\left(10+14 x+\frac{16}{3} x^{2}+\frac{16}{3 x}\right) \ln x \\
& \left.+2(1+x) \ln ^{2} x\right] .
\end{aligned}
$$

Information about the photon fragmentation function can be obtained from the process $e^{+} e^{-} \rightarrow \gamma X$. However, this process also has a large contribution from the direct coupling of photons to quarks, which dominates over the fragmentation contribution, particularly if the photon is experimentally isolated (see Sec. VI.B). The direct contribution has been used extensively to extract the electroweak couplings of up- and down-type quarks to the $Z$ boson by the ALEPH (Decamp et al., 1991; Buskulic et al., 1993), DELPHI (Abreu et al., 1995), L3 (Adriani et al., 1992, 1993), and OPAL (Akrawy et al., 1990; Alexander et al., 1991; Acton et al., 1993) Collaborations.

In the $\overline{\mathrm{MS}}$ scheme the NLO inclusive photon cross section is given by (Altarelli et al., 1979)

$$
\begin{aligned}
\frac{1}{\sigma_{0}} \frac{d \sigma\left(Q^{2}\right)}{d x}= & \sum_{q} 2 e_{q}^{2}\left(D_{\gamma / q}\left(Q^{2}\right)+\frac{\alpha}{2 \pi} e_{q}^{2} C_{\gamma}^{T}\right. \\
& +\frac{\alpha_{s}\left(Q^{2}\right)}{2 \pi}\left[C_{q}^{T} \otimes D_{\gamma / q}\left(Q^{2}\right)+C_{g}^{T}\right. \\
& \left.\left.\otimes D_{\gamma / g}\left(Q^{2}\right)\right]\right),
\end{aligned}
$$

where $\sigma_{0}=4 \pi \alpha^{2} N_{C} /\left(3 Q^{2}\right)$ is $N_{C}$ times the pointlike cross section for $e^{+} e^{-} \rightarrow \mu^{+} \mu^{-}$, the factor of 2 comes from $D_{\gamma / q}\left(Q^{2}\right)=D_{\gamma / \bar{q}}\left(Q^{2}\right)$, and the timelike Wilson co- efficients for the transverse and longitudinal cross sections are given by Eqs. (5.7). The photonic Wilson coefficients are $C_{\gamma, i}^{T}=C_{g, i}^{T} / C_{F}$. Here $C_{\gamma, T}^{T}$ contains a term $\propto \ln \left[x^{2}(1-x)\right]$ with a logarithmic singularity at $x=1$ and a stronger singularity at $x=0$. Glück, Reya, and Vogt (1993) defined a modified DIS ${ }_{\gamma}$ scheme for the photon fragmentation function by absorbing the transverse photonic Wilson coefficient $C_{\gamma, T}^{T}$ into the quark fragmentation function $D_{\gamma / q}^{\mathrm{DIS}_{\gamma}}\left(Q^{2}\right)=D_{\gamma / q}^{\overline{\mathrm{MS}}}\left(Q^{2}\right)+\alpha /(2 \pi) e_{q}^{2} C_{\gamma, T}^{T}$. This again affects the NLO photon splitting functions,

$$
\begin{aligned}
& P_{\gamma \leftarrow q}^{\mathrm{DIS}_{\gamma}}=P_{\gamma \leftarrow q}^{\overline{\mathrm{MS}}}-e_{q}^{2} P_{q \leftarrow q} \otimes C_{\gamma, T}^{T}, \\
& P_{\gamma \leftarrow g}^{\mathrm{DIS}}=P_{\gamma \leftarrow g}^{\overline{\mathrm{MS}}}-2 \sum_{q} e_{q}^{2} P_{q \leftarrow g} \otimes C_{\gamma, T}^{T} .
\end{aligned}
$$

In leading order of $\alpha$, the third evolution equation in Eqs. (6.1) can be directly integrated with the result $D_{\gamma / \gamma}\left(x, Q^{2}\right)=\delta(1-x)$. Furthermore, in leading order of $\alpha_{s}$ only the evolution equation of the quark-photon fragmentation function

$$
\frac{d D_{\gamma / q}\left(x, Q^{2}\right)}{d \ln Q^{2}}=\frac{\alpha}{2 \pi} P_{\gamma \leftarrow q}(x)
$$

survives, which can also be integrated with the result (Gehrmann-de Ridder, Gehrmann, and Glover, 1997)

$$
D_{\gamma / q}\left(x, Q^{2}\right)=\frac{\alpha}{2 \pi} P_{\gamma \leftarrow q}(x) \ln \frac{Q^{2}}{Q_{0}^{2}}+D_{\gamma / q}\left(x, Q_{0}^{2}\right) .
$$

The first term in Eq. (6.6) is the perturbatively calculable pointlike solution, while the second term is a hadronic boundary condition that must be fitted to experimental data, e.g., on $e^{+} e^{-} \rightarrow \gamma+$ jet. Following this approach the ALEPH Collaboration obtained the leading-order result (Buskulic et al., 1996)

$$
D_{\gamma / q}\left(x, Q_{0}^{2}\right)=\frac{\alpha}{2 \pi}\left[-P_{\gamma \leftarrow q}(x) \ln (1-x)^{2}-13.26\right]
$$

at the starting scale $Q_{0}=0.14 \mathrm{GeV}$. For the fragmentation function of quarks to photons with virtuality $P^{2}$, the purely perturbative leading-order result is (Qiu and Zhang, 2001; Braaten and Lee, 2002)

$$
\begin{aligned}
D_{\gamma / q}\left(x, Q^{2}, P^{2}\right)= & e_{q}^{2} \frac{\alpha}{2 \pi}\left[\frac{1+(1-x)^{2}}{x} \ln \frac{x Q^{2}}{P^{2}}\right. \\
& \left.-z\left(1-\frac{P^{2}}{x Q^{2}}\right)\right] .
\end{aligned}
$$

The NLO evolution equation for the quark-photon fragmentation function can also be integrated, if one neglects higher-order contributions from the gluon-photon fragmentation function, the mixing between different quark flavors, and the running of $\alpha_{s}\left(Q^{2}\right)$. The result, 
TABLE XIV. Parametrizations of the photon fragmentation functions. The coherence of the vectormeson-dominance (VMD) model is determined by the coefficients $e_{u}$ and $e_{d}$ in Eq. (3.16). ACFGP and GRV relate the photon to the pion, while BFG fit $\rho$ production data from ALEPH and HRS.

\begin{tabular}{|c|c|c|c|c|c|c|c|}
\hline Group & Year & Set & $\begin{array}{c}Q_{0}^{2} \\
\left(\mathrm{GeV}^{2}\right)\end{array}$ & $\begin{array}{l}\text { Factor. } \\
\text { scheme }\end{array}$ & $\begin{array}{l}\text { VMD } \\
\text { model }\end{array}$ & $N_{f}$ & $\begin{array}{l}\Lambda_{\overline{\mathrm{MS}}}^{N_{f}=4} \\
(\mathrm{MeV})\end{array}$ \\
\hline ACFGP & 1993 & NLO & 2.00 & $\overline{\mathrm{MS}}$ & coherent & 4 & 230 \\
\hline \multirow[t]{2}{*}{ GRV } & 1993 & LO & 0.25 & LO & incoherent & 5 & 200 \\
\hline & & NLO & 0.30 & $\mathrm{DIS}_{\gamma}$ & & & 200 \\
\hline BFG & 1998 & NLO & 0.50 & $\overline{\mathrm{MS}}$ & coherent & 4 & 230 \\
\hline
\end{tabular}

$$
\begin{aligned}
D_{\gamma / q}\left(x, Q^{2}\right)= & \frac{\alpha}{2 \pi} P_{\gamma \leftarrow q}(x) \ln \left(\frac{Q^{2}}{Q_{0}^{2}}\right) \\
& +D_{\gamma / q}\left(x, Q_{0}^{2}\right)+\frac{\alpha_{s}}{2 \pi} P_{q \leftarrow q}(x) \ln \left(\frac{Q^{2}}{Q_{0}^{2}}\right) \\
& \otimes\left[\frac{\alpha}{2 \pi} \frac{1}{2} P_{\gamma \leftarrow q}(x) \ln \left(\frac{Q^{2}}{Q_{0}^{2}}\right)+D_{\gamma / q}\left(x, Q_{0}^{2}\right)\right],
\end{aligned}
$$

is then exact at the fixed order $\mathcal{O}\left(\alpha \alpha_{s}\right)$, but does not take into account the usual resummation of powers of $\ln \left(Q^{2} / Q_{0}^{2}\right)$. The ALEPH Collaboration has fitted its $e^{+} e^{-} \rightarrow \gamma+$ jet data in next-to-leading order, taking $\alpha_{s}$ $=0.124$ in order to reproduce the observed total rate of $e^{+} e^{-} \rightarrow$ hadrons, and obtain (Buskulic et al., 1996)

$$
\begin{aligned}
D_{\gamma / q}\left(x, Q_{0}^{2}\right)= & \frac{\alpha}{2 \pi}\left[-P_{\gamma \leftarrow q}(x) \ln (1-x)^{2}\right. \\
& +20.8(1-x)-11.07]
\end{aligned}
$$

at $Q_{0}=0.64 \mathrm{GeV}$.

The fully resummed solution of the evolution equations consists of pointlike and hadronic contributions $D_{\gamma / i}\left(Q^{2}\right)=D_{\gamma / i}^{\mathrm{pl}}\left(Q^{2}\right)+D_{\gamma / i}^{\mathrm{had}}\left(Q^{2}\right)$. The full leading-order (Duke and Owens, 1982) and NLO pointlike solutions (Aurenche, Chiappetta, et al., 1993; Bourhis, Fontannaz, and Guillet, 1998)

$$
D_{\gamma / i}^{\mathrm{pl}}\left(Q^{2}\right)=\frac{\alpha}{2 \pi}\left[\frac{4 \pi}{\alpha_{s}\left(Q^{2}\right)} a_{i}+b_{i}+\mathcal{O}\left(\alpha_{s}\right)\right]
$$

can only be calculated analytically in moment space. The hadronic input can unfortunately not be determined from inclusive photon production in $e^{+} e^{-}$annihilation, since the experimental data (Buskulic et al., 1996; Ackerstaff et al., 1998) are very limited and are furthermore dominated by the pointlike quark-photon fragmentation function. Therefore all existing parametrizations [Aurenche, Chiappetta, et al. (ACFGP), 1993; Glück, Reya, and Vogt (GRV), 1993; Bourhis, Fontannaz, and Guillet (BFG), 1998] use vector-meson dominance to model the photon fragmentation at low scales. The different input parameters are summarized in Table XIV. Heavy quarks are included above their production thresholds with boundary conditions $D_{\gamma / h}\left(x, m_{h}^{2}\right)=D_{\gamma / \bar{h}}\left(x, m_{h}^{2}\right)=0$.

Figure 45 demonstrates that the most recent data from OPAL (Ackerstaff et al., 1998) can be described by the pointlike fragmentation functions in leading order (long-dashed curve) and next-to-leading order (dotted curve). The data thus cannot discriminate between the different leading-order (solid curve) and NLO (shortdashed and dot-dashed curves) assumptions for the hadronic input.

\section{B. Isolation}

Photons produced via fragmentation usually lie inside hadronic jets, while directly produced photons tend to be isolated from the final-state hadrons. The theoretical uncertainty coming from the nonperturbative fragmentation function can therefore be reduced if the photon is isolated in phase space. At the same time the experimental uncertainty coming from photonic decays of $\pi^{0}$, $\eta$, and $\omega$ mesons is considerably reduced. Photon isolation can be achieved by limiting the (transverse) hadronic energy $E_{(T)}^{\text {had }}$ inside a cone of size $R$ around the photon to

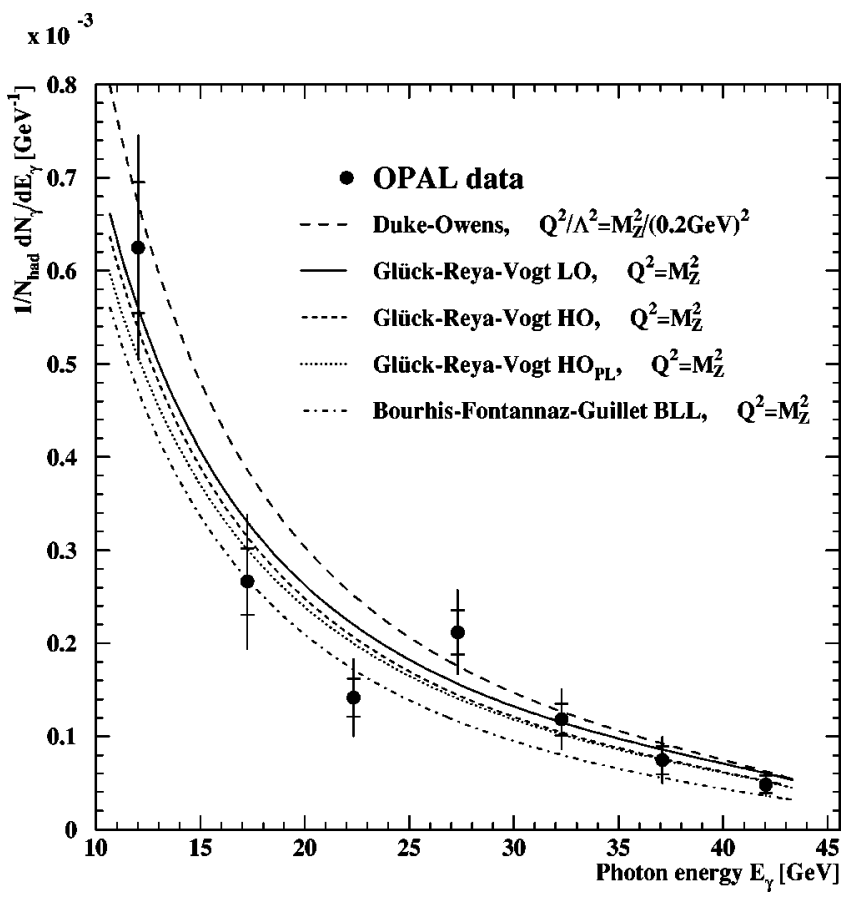

FIG. 45. Parametrizations of the photon fragmentation function at $Q^{2}=M_{Z}^{2}$ compared to OPAL data. From Ackerstaff et al., 1998. 


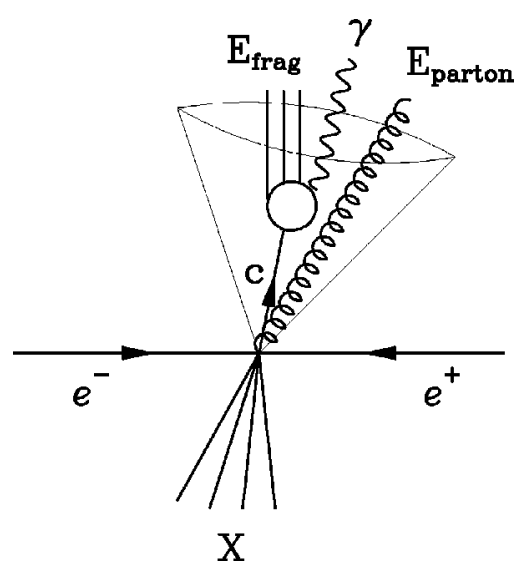

FIG. 46. Illustration of an isolation cone containing a parton $c$ that fragments into a photon $\gamma$ plus hadronic energy $E_{\text {frag }}$. In addition, a gluon enters the cone and fragments, giving hadronic energy $E_{\text {parton }}$. From Berger, Guo, and Qiu, 1996.

$$
E_{(T)}^{\mathrm{had}}<\epsilon_{(T)} E_{(T), \gamma} .
$$

This is illustrated in Fig. 46. For small photon pseudorapidities $\eta$, the cone size $R=\delta / \cosh \eta$ is approximately equal to the half angle of the cone $\delta$. Since in next-toleading order the direct and fragmentation processes are intimately linked at the factorization scale $Q$, it is mandatory that the isolation criterion not interfere with the cancellation of soft and collinear singularities. For certain differential cross sections like $d \sigma / d x_{\gamma}$ in $e^{+} e^{-}$annihilation, where $x_{\gamma}=2 E_{\gamma} / Q^{2}$ is the fractional photon energy, spurious infrared singularities remain at the point $x_{\gamma}=1 /(1+\epsilon)$ (Berger, Guo, and Qiu, 1996). The cross section $d \sigma / d x_{\gamma}$ is therefore defined only as a distribution, which has to be integrated with a test function over finite bin widths. Hence for physical observables these integrable singularities disappear. Nevertheless, the cross section contains logarithms of the type $\ln [1$ $\left.-x_{\gamma}(1+\epsilon)\right]$, which can become large at $x_{\gamma}=1 /(1+\epsilon)$ (Aurenche, Fontannaz, et al., 1997; Catani, Fontannaz, and Pilon, 1998). For physical observables it is therefore important to integrate over large enough bins in $x_{\gamma}$. The situation is very similar to the infrared sensitivity of the NLO dijet cross section at large values of the initial-state photon energy fraction $x_{\gamma}^{\text {obs }}$. Recently an improved photon isolation criterion,

$$
\sum_{i} E_{(T), i}^{\mathrm{had}} \theta\left(\delta-R_{i}\right)<\epsilon_{(T)} E_{(T), \gamma}\left(\frac{1-\cos \delta}{1-\cos \delta_{0}}\right),
$$

has been proposed, where $\delta \leqslant \delta_{0}$ and $\delta_{0}$ is now the isolation cone (Frixione, 1998a). This procedure allows the fragmentation contribution to vanish in an infrared-safe way.

\section{Inclusive photons}

The photoproduction of inclusive photons proceeds through direct and resolved initial-state photons and through direct and fragmentation production of the final-state photon. Thus four types of partonic subpro-

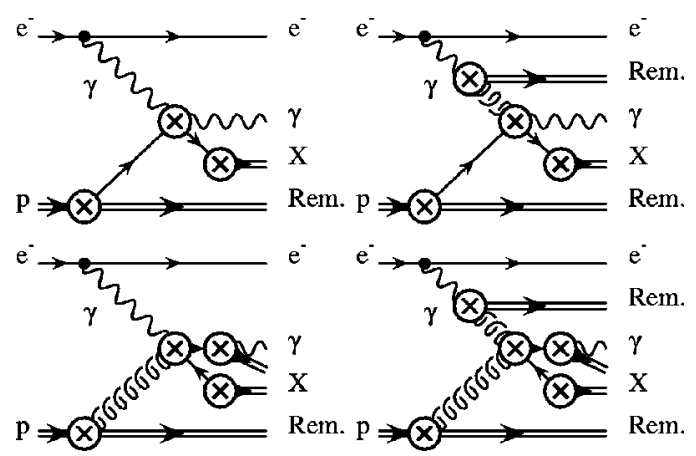

FIG. 47. Factorization of lepton-hadron scattering into photons.

cesses contribute to $\gamma h \rightarrow \gamma X$, as shown in Fig. 47, and eight types contribute to $\gamma \gamma \rightarrow \gamma X$. The hadronic cross section is given by Eq. (5.14), where the hadron fragmentation function is replaced by the photon fragmentation function $D_{\gamma / c}$. For direct photon production the fragmentation function is, of course, simply $\delta(1-z)$, and the cross section coincides with Eq. (4.14).

The partonic leading-order diagrams and cross sections can be obtained by crossing the diagrams in Figs. 7-9 and the matrix elements in Table VI, and the NLO corrections are calculated as outlined in Sec. V.B. The only additional subtlety occurs when a final-state quark splits into a quark and a photon. The corresponding collinear singularity,

$$
\begin{aligned}
\bar{\Gamma}_{\gamma \leftarrow q}\left(x, \mu^{2}\right)= & \delta_{\gamma q} \delta(1-x)-\frac{1}{\varepsilon} \frac{\alpha}{2 \pi} \frac{\Gamma(1-\varepsilon)}{\Gamma(1-2 \varepsilon)}\left(\frac{4 \pi \mu^{2}}{Q^{2}}\right)^{\varepsilon} \\
& \times P_{\gamma \leftarrow q}(x)+\mathcal{O}\left(\varepsilon, \alpha^{2}, \alpha \alpha_{s}\right) \\
= & \delta_{\gamma q} \delta(1-x)-\left(\frac{1}{\varepsilon}-\gamma_{E}+\ln (4 \pi)+\ln \frac{\mu^{2}}{Q^{2}}\right) \\
& \times \frac{\alpha}{2 \pi} P_{\gamma \leftarrow q}(x)+\mathcal{O}\left(\varepsilon, \alpha^{2}, \alpha \alpha_{s}\right),
\end{aligned}
$$

is absorbed into the photon fragmentation function

$$
D_{\gamma / q}\left(x, M_{f}^{2}\right)=\bar{D}_{\gamma / q}(x)+\left[\bar{\Gamma}_{\gamma \leftarrow q}\left(M_{f}^{2}\right) \otimes \bar{D}_{\gamma / \gamma}\right](x)
$$

at the factorization scale $M_{f}^{2}$.

NLO corrections to resolved-direct and resolvedfragmentation processes have been calculated in the context of prompt-photon production in hadronic collisions by different groups (see Sec. VII.C), while those for the direct-fragmentation process have been calculated in the context of inclusive hadron photoproduction (see Sec. V.B). The NLO corrections, which are specific only for the photoproduction of prompt photons, i.e., the corrections to the direct-direct process, have been calculated for inclusive photons by Duke and Owens (1982), Aurenche, Baier, et al. (1984a), Bawa, Krawczyk, and Stirling (1991), and Gordon and Storrow (1994). Full NLO calculations for photon-hadron scattering have been performed by Gordon and Vogelsang (1995), using a subtraction term in order to account also for photon isolation, and by Fontannaz, Guillet, and Hein- 


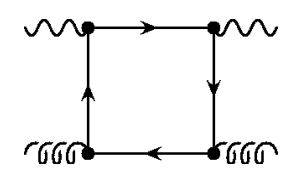

FIG. 48. The quark box contribution to direct photon photoproduction.

rich (2001a) in a completely differential form. Gordon and Storrow (1996) have furthermore calculated NLO corrections to prompt-photon production in photonphoton collisions.

The direct process $\gamma \gamma \rightarrow \gamma X$ can only proceed through a quark box diagram with $X=\gamma$ and is of $\mathcal{O}\left(\alpha^{4}\right)$. The process $\gamma g \rightarrow \gamma X$ with $X=g$ proceeds through the quark box shown in Fig. 48 and similar diagrams with reversed and crossed fermion flow and is of $\mathcal{O}\left(\alpha^{2} \alpha_{s}^{2}\right)$. It thus represents a NNLO correction to the tree-level subprocesses that contain at least one quark line and are of $\mathcal{O}\left(\alpha^{2}\right)$. Since the box contribution is by itself gauge invariant and can be numerically large, it can and must be included together with the NLO corrections of the treelevel processes (Aurenche, Baier, et al., 1984a; Fontannaz, Guillet, and Heinrich, 2001a; Krawczyk and Zembrzuski, 2001a).

In Fig. 49 the only existing complete NLO calculation for isolated photons, which also includes the NNLO box contribution (Fontannaz, Guillet, and Heinrich, 2001a), is compared to recent data from ZEUS (Breitweg et al., 2000d). The transverse momentum $\left(p_{T}^{\gamma}\right)$ distribution (top) agrees quite well with the data, but the experimental errors are still too large to distinguish between the AFG and GRV parton densities in the photon. On the other hand, the NLO pseudorapidity $\left(\eta^{\gamma}\right)$ distributions with AFG photon densities (bottom), integrated over $p_{T}^{\gamma} \in[5 ; 10] \mathrm{GeV}$, show some discrepancies with the data: The calculations underestimate the data for negative rapidities and small fractional photon energies $y$ $=E_{\gamma} / E_{e}$ and overestimate them for positive rapidities and large photon energies. Similar results are obtained with the calculations of Gordon (1998) and Krawczyk and Zembrzuski (2001a), which are partly based on leading-order cross sections. The movement of the maximum towards the backward direction is caused by an increase of the photon energy participating in the hard scattering process. A possible explanation for the discrepancy in the forward direction might be that there is more hadronic activity in this experimental region, which is more strongly affected by the isolation cut than the theoretical parton-level simulation. At a future THERA collider, where $250-\mathrm{GeV}$ electrons would be collided with $920-\mathrm{GeV}$ protons, the cross section in the ZEUS kinematic region would be about three times larger (Krawczyk and Zembrzuski, 2001b).

\section{Photons and jets}

Prompt-photon production in association with an observed jet has been calculated by Gordon (1998) and by Krawczyk and Zembrzuski (2001a). However, both of
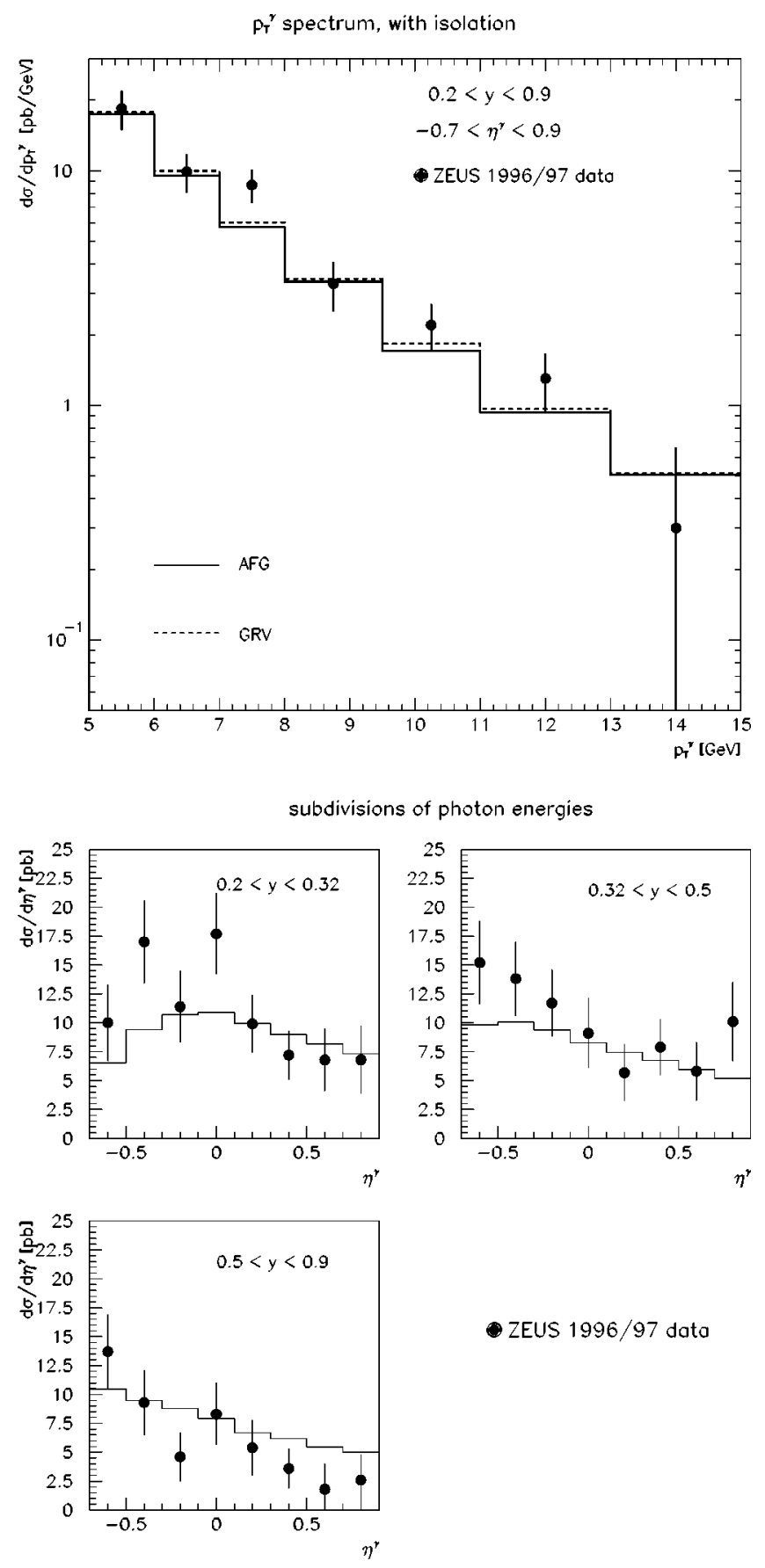

ZEUS $1996 / 97$ dato

FIG. 49. Transverse momentum (top) and pseudorapidity (bottom) spectra of isolated photons in next-to-leading order compared to ZEUS data. From Fontannaz, Guillet, and Heinrich, 2001a.

these calculations use leading-order expressions for the resolved-fragmentation contribution (the latter also for the direct-fragmentation and resolved-direct contributions). Numerical results of these calculations, using GRV and GS parton densities in the photon, are compared with ZEUS data (Lee et al., 2000) and the Monte Carlo predictions of PYTHIA (Sjöstrand, 1994) and HERWIG (Abbiendi et al., 1992) in Fig. 50. The shape of the transverse energy distribution (top), integrated over $\eta^{\gamma} \in[-0.7 ; 0.9]$, is described by all theoretical predictions, but the magnitude is not quite reproduced by the 


\section{ZEUS 1996-97 preliminary}

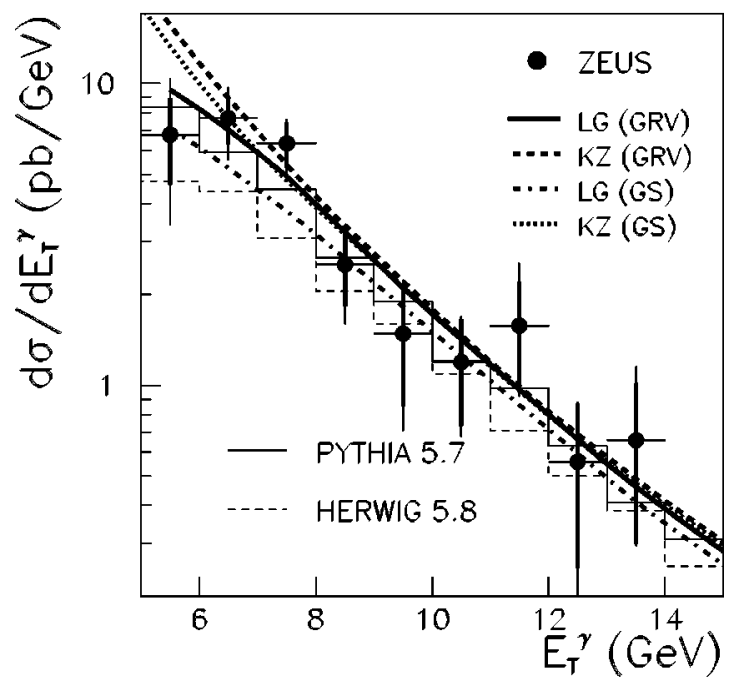

ZEUS $1996-97$ preliminary

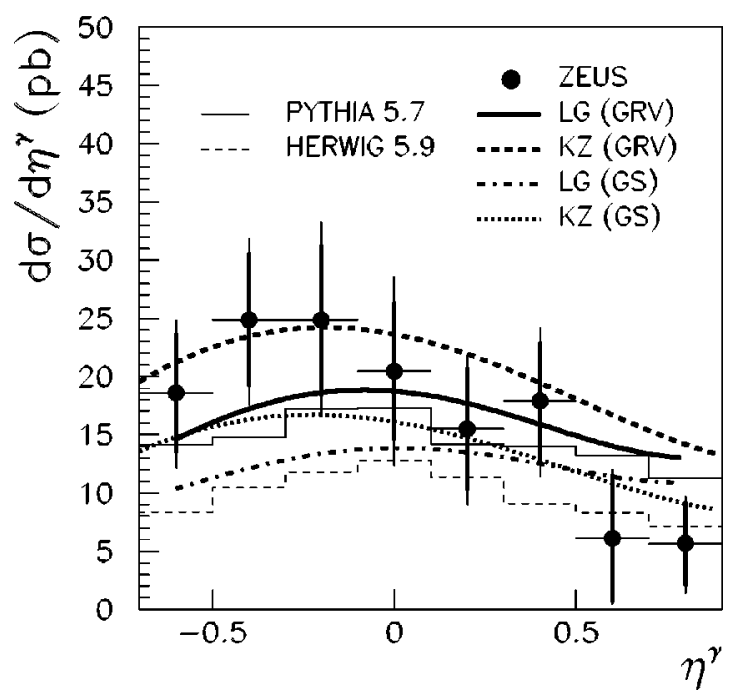

FIG. 50. Transverse energy (top) and pseudorapidity (bottom) spectra of isolated photons produced in association with a jet of $E_{T}>5 \mathrm{GeV},-1.5<\eta<1.8$, and cone size $R=1$ compared to ZEUS data. From Lee, 2000.

Monte Carlo predictions. The same observation can be made in the pseudorapidity distribution (bottom) integrated over $E_{T}^{\gamma} \in[5 ; 10] \mathrm{GeV}$, but here the higher-order calculations with GS photon densities also fall below the data in the backward region. The main difference between the two higher-order calculations comes from the box contribution, which is included only in the calculation of Krawczyk and Zembrzuski (2001a).

Fixed-target and hadron collider experiments indicate that prompt-photon production may be influenced by intrinsic transverse momenta of the scattering initial partons (see Sec. VII.C). The ZEUS Collaboration has analyzed isolated prompt-photon photoproduction in association with a jet and observed an excess over the leading-order QCD expectation in the distributions of perpendicular momentum $p_{\perp}$, longitudinal momentum $p_{\|}$, and azimuthal angle $\Delta \phi$ of the photon relative to the balancing jet (Chekanov et al., 2001). They attributed these discrepancies to an effective $\left\langle k_{T}\right\rangle$ of 1.69 $\pm 0.18_{-0.20}^{+0.18} \mathrm{GeV}$, which includes effects coming from the initial-state parton showering as modeled within PYTHIA (Sjöstrand, 1994). This value of $\left\langle k_{T}\right\rangle$ seems to be consistent with determinations in hadron collisions at different energies, which are, however, obtained using a variety of methods. Within the leading-order Monte Carlo comparison, the ZEUS data seem to support the trend that the effective $\left\langle k_{T}\right\rangle$ in the proton rises with the available hadronic energy. However, the data have recently been confronted with a calculation for photon plus jet photoproduction that includes all NLO corrections and the NNLO box diagram (Fontannaz, Guillet, and Heinrich, 2001b). The comparison is complicated by the fact that equal cuts on the transverse energies of the photon and recoiling jet were used in the experiment $\left(E_{T}\right.$ $>5 \mathrm{GeV}$ ), which must be relaxed in the NLO calculation and induces some sensitivity on the size of the mismatch $\left|E_{T \text {,min }}^{\gamma}-E_{T \text {,min }}^{\text {jet }}\right|<0.5 \mathrm{GeV}$. The sensitivity within this range is, however, smaller than the experimental uncertainty. Within errors, the full NLO calculation can describe the ZEUS data without any intrinsic $\left\langle k_{T}\right\rangle$ effects. The production of direct photons and jets in direct, single-, and double-resolved photon-photon collisions has also recently been calculated in next-to-leading order (Fontannaz, Guillet, and Heinrich, 2002), and good agreement with preliminary OPAL data (Abbiendi et al., 2001d) was found. In the future, NLO calculations should be performed for the production of two photons and photons in association with light and heavy hadrons in order to obtain more information about the flavor content of photons and protons.

\section{RELATED TOPICS}

In this section, the topics discussed so far will be extended in several directions. First, the photon virtuality, which so far has been assumed to be negligible, will be explicitly taken into account. Second, the initial photon will be allowed to have a well-defined polarization. Third, the production of prompt photons in hadron collisions will be discussed; this is closely related to photoproduction by crossing an initial-state photon into the final state.

\section{A. Virtual photons}

If the momentum transfer in a lepton scattering process $q=p_{l}-p_{l^{\prime}}$ is small but not completely negligible, the corresponding cross section can be calculated in the equivalent-photon approximation using a differential form of the transverse photon flux (Budnev et al., 1974; Kessler, 1975),

$$
\frac{d f_{\gamma / l}^{\text {brems }}}{d P^{2}}\left(x, P^{2}\right)=\frac{\alpha}{2 \pi}\left[\frac{1+(1-x)^{2}}{x} \frac{1}{P^{2}}-\frac{2 m_{l}^{2} x}{P^{4}}\right] .
$$

The Weizsäcker-Williams approximation [Eq. (2.1)] is 


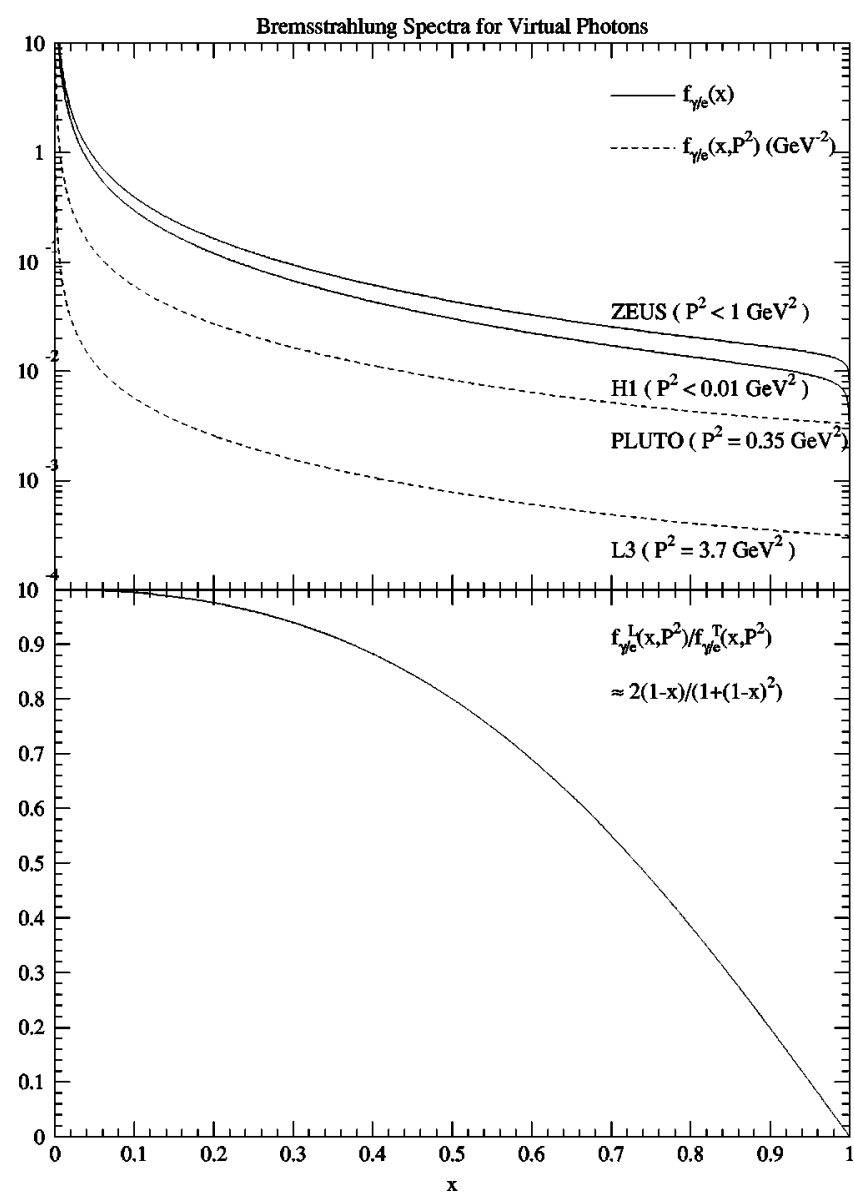

FIG. 51. Bremsstrahlung spectra for transverse photons with different virtualities (top) and the ratio of longitudinal and transverse photon fluxes (bottom). The latter is essentially independent of the photon virtuality.

recovered by integrating Eq. (7.1) over the photon virtuality $P^{2}=-q^{2}$. As $P^{2}$ becomes larger, the longitudinal photon flux

$$
\frac{d f_{\gamma / l}^{\text {brems }}}{d P^{2}}\left(x, P^{2}\right)=\frac{\alpha}{2 \pi}\left[\frac{2(1-x)}{x} \frac{1}{P^{2}}\right]
$$

must also be taken into account. Bremsstrahlung spectra for transverse photons with different virtualities are shown in Fig. 51 (top) together with the ratio of longitudinal and transverse photon fluxes (bottom), which is essentially independent of the photon virtuality.

Like real photons, photons with a nonzero virtuality $P^{2}$ can be probed for their hadronic structure in deepinelastic electron-photon scattering. As $P^{2}$ approaches the virtuality $Q^{2}$ of the probing photon, the structure of the virtual photon reduces to the perturbative splitting of the photon into a collinear quark-antiquark pair with probability (Uematsu and Walsh, 1981, 1982; Rossi, 1984)

$$
\begin{aligned}
f_{q / \gamma}^{\text {box }}\left(x, Q^{2}, P^{2}\right)= & 3 e_{q}^{2} \frac{\alpha}{2 \pi}\left(\left[x^{2}+(1-x)^{2}\right] \ln \frac{Q^{2}}{x^{2} P^{2}}\right. \\
& +6 x(1-x)-2) .
\end{aligned}
$$

This expression includes contributions from transverse and longitudinal photons. It differs from the real-photon case $f_{q / \gamma}\left(x, Q^{2}\right)=e_{q}^{2} \alpha C_{\gamma} /(2 \pi)$ with $C_{\gamma}$ given in Eq. (3.11), since the collinear singularity is now mass regulated by the photon virtuality $P^{2}$. In the limit $P^{2} \ll Q^{2}$, the structure of the virtual photon should, of course, coincide with that of the real photon.

A simple phenomenological ansatz to suppress smoothly the quark and gluon content in the virtual photon at high $P^{2}$ is (Drees and Godbole, 1994)

$$
\begin{array}{r}
f_{q / \gamma}\left(x, Q^{2}, P^{2}\right)=f_{q / \gamma}\left(x, Q^{2}\right) \frac{\ln \frac{Q^{2}+P_{c}^{2}}{P^{2}+P_{c}^{2}}}{\ln \frac{Q^{2}+P_{c}^{2}}{P_{c}^{2}}} \\
f_{g / \gamma}\left(x, Q^{2}, P^{2}\right)=f_{g / \gamma}\left(x, Q^{2}\right) \frac{\ln ^{2} \frac{Q^{2}+P_{c}^{2}}{P^{2}+P_{c}^{2}}}{\ln ^{2} \frac{Q^{2}+P_{c}^{2}}{P_{c}^{2}}},
\end{array}
$$

where the typical hadronic scale $P_{c}$ lies between the QCD scale $\Lambda$ and the proton mass. Since gluons are radiated from off-shell quarks, they must be more suppressed than quarks (Borzumati and Schuler, 1993). A similar ansatz has also been used by Aurenche, Fontannaz, et al. (1994b). It can be applied to any parametrization for the parton densities in the real photon, which are recovered in the limit $P^{2} \ll Q^{2}$. At large $P^{2}$, the evolved parton densities are globally suppressed. This means, however, that the perturbative result of Eq. (7.3) is not reproduced, since the logarithmic suppression factors are independent of $x$.

A refined version of the above prescription has been formulated by Schuler and Sjöstrand (SaS; 1995, 1996), who sum the real hadronic contributions from the three dominant vector mesons $\rho, \omega$, and $\phi$ after weighting them with factors $\eta\left(P^{2}\right)=\left(1+P^{2} / m_{\rho, \omega, \phi}^{2}\right)^{-2}$ and evolving them from $\max \left(P^{2}, Q_{0}^{2}\right)$. The pointlike contribution is evolved with a continuous suppression factor (1 $\left.+P^{2} / k^{2}\right)^{-2}$, so that it also starts effectively from $\max \left(P^{2}, Q_{0}^{2}\right)$. In the low- and high- $P^{2}$ limits, the realphoton densities and the logarithmically enhanced terms of the virtual pointlike box are correctly reproduced, but not the $x$-dependent terms of the latter.

Glück, Reya, and Stratmann (GRSt; 1995) interpret Eq. (7.3) at $P^{2}=Q^{2}$ and $f_{g / \gamma}\left(x, P^{2}, P^{2}\right)=0$ as NLO pointlike boundary conditions. In leading order these conditions differ, since here also $f_{q / \gamma}\left(x, P^{2}, P^{2}\right)=0$. The pointlike boundary conditions are then added to the hadronic VMD input for real photons $f_{i / \gamma}^{\text {had }}\left(x, Q_{0}^{2}\right)$, adopted from Glück, Reya, and Vogt (GRV; 1992b), with an interpolating factor $\eta\left(P^{2}\right)=\left(1+P^{2} / m_{\rho}^{2}\right)^{-2}$, so that

$$
\begin{aligned}
& f_{i / \gamma}\left(x, \max \left(P^{2}, Q_{0}^{2}\right), P^{2}\right) \\
&= \eta\left(P^{2}\right) f_{i / \gamma}^{\mathrm{had}}\left(x, \max \left(P^{2}, Q_{0}^{2}\right)\right) \\
&+\left[1-\eta\left(P^{2}\right)\right] f_{i / \gamma}^{\mathrm{box}}\left(x, \max \left(P^{2}, Q_{0}^{2}\right), P^{2}\right) .
\end{aligned}
$$


The functional form of $\eta\left(P^{2}\right)$ can be derived from a dispersion integral in the photon virtuality, which is dominated by the poles of the vector mesons, particularly the $\rho$ meson, in the region of small $P^{2}$. In the limit $P^{2} \ll Q^{2}$, the purely hadronic real-photon input is recovered in leading order and next-to-leading order, as required by the DIS $\gamma$ scheme. As $P^{2}$ approaches $Q^{2}$, the unevolved initial parton densities are globally suppressed and the virtual pointlike result, Eq. (7.3), is recovered. This is, however, inconsistent with the original DIS $_{\gamma}$ scheme, since the NLO pointlike quark boundary condition now differs from zero, but is evolved using the same massless DIS $_{\gamma}$ splitting functions [Eqs. (3.13)] in the inhomogeneous evolution equations [Eqs. (3.5)] from the new starting scale $\max \left(P^{2}, Q_{0}^{2}\right)$. A parametrization of these virtual-photon parton densities, which is valid in the region $P^{2}<Q^{2} / 5$, has been performed only in leading order (Glück, Reya, and Stratmann, 1996).

The inconsistencies with the DIS ${ }_{\gamma}$ scheme can be avoided if the pointlike term in Eq. (7.5) is omitted [Glück, Reya, and Schienbein (GRSc), 1999b, 2001]. However, in this case the perturbative splitting Eq. (7.3) is not reproduced. Consequently this virtual parametrization is only applicable in the region of small $P^{2}\left(P^{2}\right.$ $<Q^{2} / 10$ ), where the virtuality of the pointlike term is of minor importance, and the large- $P^{2}$ region should be calculated in fixed order. A parametrization has also been given only in leading order, although the NLO results are very similar.

The existing NLO parton densities in the transverse virtual photon are compared in Fig. 52 at $Q^{2}$ $=25 \mathrm{GeV}^{2}$ and $P^{2}=0.5 \mathrm{GeV}^{2}$ (left) and $2.5 \mathrm{GeV}^{2}$ (right) for the up-quark (top), down-quark (center), and gluon (bottom) densities. The suppression of the realphoton GRV densities in the virtual photon is clearly visible. For the quarks (gluons) it is weakest (strongest) for the global Drees-Godbole factors, for which $P_{c}^{2}$ $=0.3 \mathrm{GeV}^{2}$ has been used. At $P^{2}=Q^{2} / 50$ the $x$ dependence is only weakly modified, while at $P^{2}=Q^{2} / 10$ the logarithmic singularity at $x=0$ in the perturbative box is significantly weaker. The different parametrizations vary largely at small $P^{2}$, particularly for the gluon, but much less at larger $P^{2}$ as expected.

Information about the parton densities in the virtual photon can be gained from the total hadronic cross section in double-tagged $e^{+} e^{-}$scattering in the Bjorken region $P^{2} \ll Q^{2}$. If the probing and target photons are both soft, their transverse $(T)$ and longitudinal $(L)$ polarizations contribute with equal weights to the effective virtual-photon structure function,

$$
F_{\mathrm{eff}}^{\gamma} \simeq F_{\mathrm{TT}}^{\gamma}+F_{\mathrm{TL}}^{\gamma}+F_{\mathrm{LT}}^{\gamma}+F_{\mathrm{LL}}^{\gamma} \simeq F_{2}^{\gamma}+\frac{3}{2} F_{L}^{\gamma} .
$$

In the second part of this equation, the relations $F_{\mathrm{TL}}^{\gamma}$ $\simeq F_{\mathrm{LT}}^{\gamma} \equiv F_{L}^{\gamma}$ and $F_{\mathrm{LL}}^{\gamma} \simeq 0$ have been used, which apply, strictly speaking, only to the virtual box contribution with $F_{L}^{\gamma}=\Sigma_{q} 2 x e_{q}^{2}(\alpha / 2 \pi) e_{q}^{2} N_{C} 4 x(1-x)$. Like $F_{2}^{\gamma}, F_{L}^{\gamma}$ could be calculated by inserting longitudinal virtualphoton densities into Eq. (3.12) and employing the lon-

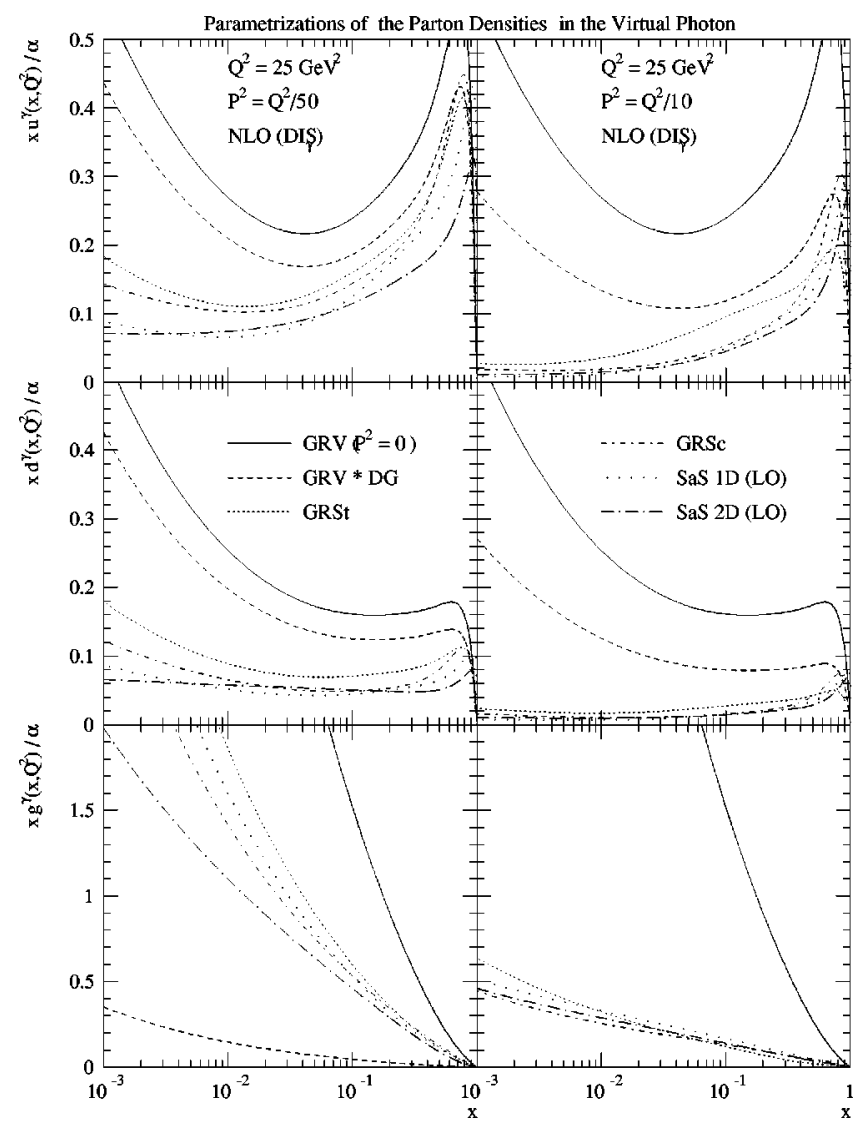

FIG. 52. NLO parametrizations of the up-quark (top), downquark (center), and gluon (bottom) densities in a photon with virtuality $P^{2}=0.5 \mathrm{GeV}^{2}$ (left) and $2.5 \mathrm{GeV}^{2}$ (right) at $Q^{2}$ $=25 \mathrm{GeV}^{2}$.

gitudinal Wilson coefficients $C_{q, L}=8 x / 3$ and $C_{g, L}$ $=2 x(1-x)$. In practice, however, only a pointlike parametrization for the longitudinal virtual photon is currently available (Chýla and Taševský, 2001), and it turns out to be very close to the unevolved longitudinal box result.

In Fig. 53, $F_{\text {eff }}^{\gamma}$ measurements by the PLUTO (top; Berger et al., 1984b) and L3 (bottom; Acciarri et al., 2000) Collaborations are compared with the virtualphoton parametrizations discussed above. In order to obtain $F_{\text {eff }}^{\gamma}$ from $F_{2}^{\gamma}$, the separately shown longitudinal virtual box contribution $3 / 2 F_{\mathrm{TL}}^{\gamma}$ has been added to all parametrizations. For the GRSc parametrization, which is evolved with three massless flavors, the sum of direct and resolved heavy-charm-quark contributions is also shown separately. While the PLUTO and L3 data tend to be higher than all parametrizations and the purely perturbative virtual box prediction, the theoretical curves all describe the measurements within the errors, so that present data do not yet convincingly establish the importance of the renormalization-group improved treatment of parton densities in the virtual photon or the need for an intrinsic hadronic component (Glück, Reya, and Schienbein, 2001).

The virtual-photon fluxes and parton densities discussed above make it possible to extend the calculations of real photoproduction processes to the region $P^{2}>0$, as long as $P^{2}$ is significantly smaller than the hard scat- 


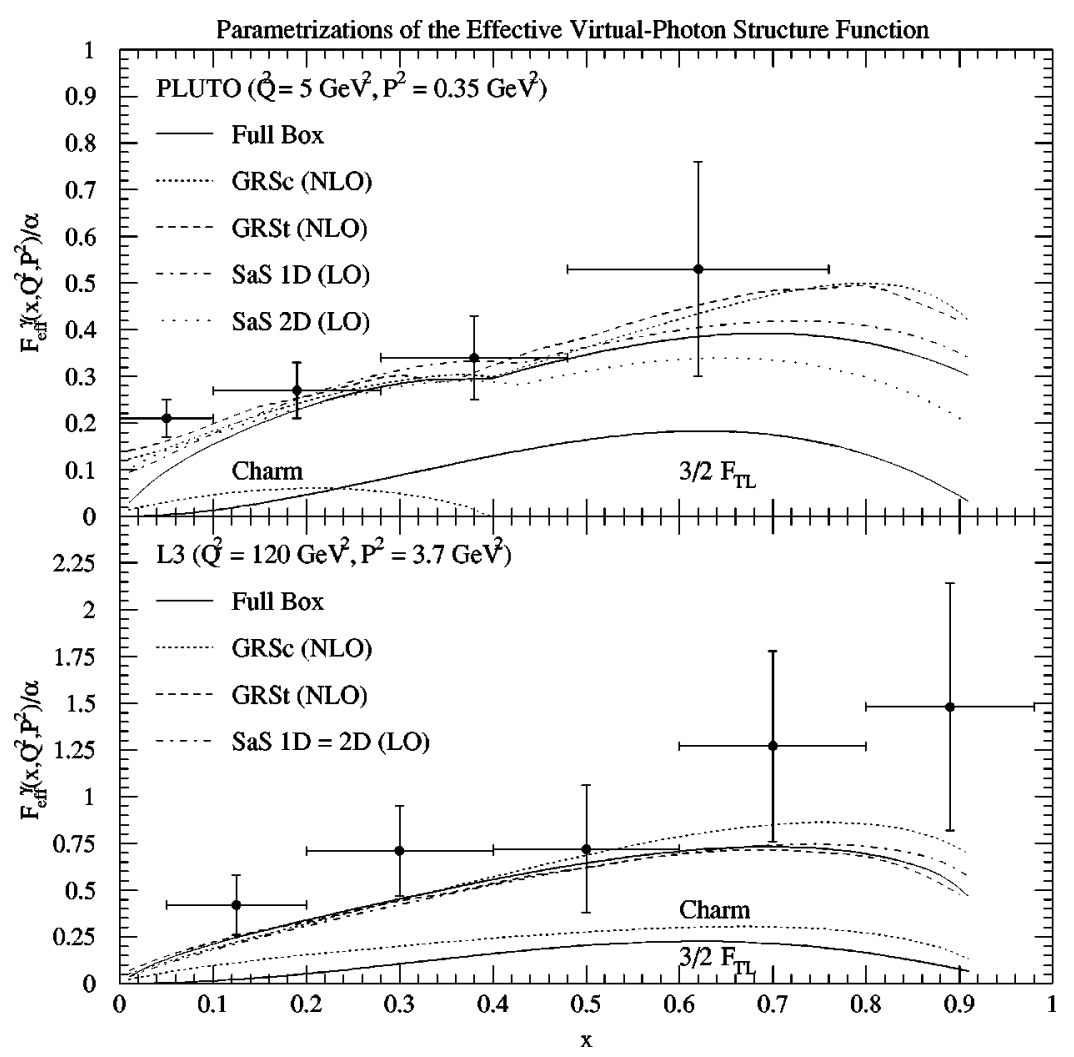

FIG. 53. Parametrizations of the effective virtual-photon structure function compared to data from PLUTO (top; Berger et al., 1984b) and L3 (bottom; Acciarri et al., 2000). tering scale $Q^{2}$ (typically the squared transverse energy $E_{T}^{2}$ of the observed jet, hadron, or prompt photon). Therefore virtual photoproduction will also receive contributions from direct and resolved photon processes. They can be calculated by taking $P^{2}$ into account only in the photon flux and parton densities, but neglecting it in the hard scattering. When $P^{2}$ approaches $Q^{2}$, however, the resolved contribution vanishes logarithmically, the longitudinal photon cross section has to be taken into account, and the deep-inelastic scattering cross section has to be recovered.

As was discussed in Sec. III, direct and resolved real photoproduction processes are related in next-to-leading order by the appearance of a collinear $1 / \varepsilon$ singularity in the splitting of the photon into quarks with fractional charge $e_{q}$ [Eq. (3.2)]. This singularity is absorbed into the renormalized quark densities in the real photon [Eq. (3.3)] and cancels its logarithmic factorization scale dependence. A similar mechanism occurs for virtual photoproduction, where the singularity is now mass regulated by $P^{2}$ and Eq. (3.2) has to be replaced with

$$
\begin{aligned}
\Gamma_{q \leftarrow \gamma^{*}}\left(x, P^{2}\right)= & \delta_{q \gamma} \delta(1-x)-\frac{\alpha}{2 \pi} \\
& \times\left(\ln \frac{P^{2}(1-x)}{Q^{2}} P_{q \leftarrow \gamma}(x)+N_{C} e_{q}^{2}\right) .
\end{aligned}
$$

This defines an $\overline{\mathrm{MS}}$ factorization scheme for virtual photons, which leaves finite terms in the hard cross section which are identical to those of real photons (Klasen, Kramer, and Pötter, 1998; Pötter, 1999c). Since there is no $1 / \varepsilon$ singularity, the subtraction is not mandatory to obtain finite cross sections, but only to resum the logarithm, which can become large for $P^{2} \ll Q^{2}$ and $x \rightarrow 1$.

In order to obtain more information about the structure of the virtual photon it is useful to consider not only inclusive electron-photon scattering, but also hard scattering processes like jet photoproduction. Leading-order studies indicate a significant contribution from resolved virtual photons at HERA, if $P^{2} \ll E_{T}^{2}$ (Glück, Reya, and Stratmann, 1996; Glück, Reya, and Schienbein, 2001). Virtual jet photoproduction has also been calculated in next-to-leading order by adapting an existing NLO calculation for resolved real photons (Klasen, Kleinwort, and Kramer, 1998) and using the $\overline{\mathrm{MS}}$ subtraction for the direct virtual-photon singularity discussed above (Klasen, Kramer, and Pötter, 1998). It has then been extended to the deep-inelastic-scattering region of large $P^{2}$ and applied to jet production at HERA (Kramer and Pötter, 1998, 1999) and LEP (Pötter, 1999a, 1999b). The resolved contribution turns out to be particularly important if the jets are produced in the forward region. The ZEUS (H1) Collaborations have measured jets with $\eta>2.6 \quad(\eta \in[1.735 ; 2.794]) \quad$ and $E_{T}>5 \mathrm{GeV} \quad\left(E_{T}\right.$ $>3.5 \mathrm{GeV}$ ), respectively (Adloff et al., 1999c; Breitweg et al., 1999b). In Fig. 54 the ZEUS data are compared to NLO predictions. While the DIS direct calculation (a) significantly underestimates the ZEUS data, they can be described (b) if resolved virtual-photon contributions from the SaS 1D fit are added (Kramer and Pötter, 1999).

In principle, the production of heavy quarks with mass $m_{h}$ is also sensitive to the structure of the virtual pho- 

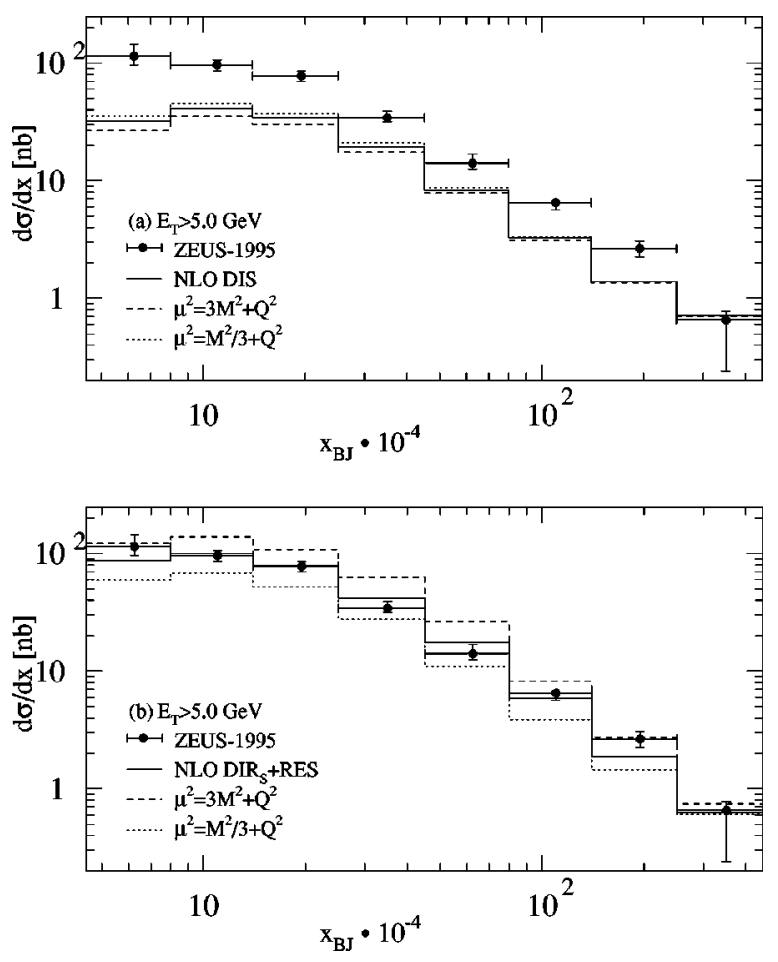

FIG. 54. NLO predictions for forward dijet production with virtual photons compared to ZEUS 1995 data: (a) the deepinelastic-scattering direct contribution; (b) direct and resolved virtual-photon contributions, added consistently (Kramer and Pötter, 1999).

ton. In this case resolved photon contributions are applicable as long as $P^{2} \ll m_{h}^{2}$. However, a leading-order study shows that they are numerically small at the relatively large values of $x$ currently probed at HERA (Glück, Reya, and Stratmann, 1996) and LEP energies (Glück, Reya, and Schienbein, 2001).

Finally, a leading-order study for virtual photoproduction of prompt photons has shown that the gluon content in the virtual photon may be tested if the photons are produced in the forward direction at transverse energies $E_{T} \simeq 5 \mathrm{GeV}$ (Krawczyk and Zembrzuski, 1998).

\section{B. Polarized photons}

Polarized photons can be produced by bremsstrahlung off lepton beams with circular polarization $\left|\lambda_{l}\right| \leqslant 1 / 2$. The corresponding spectrum $\Delta f_{\gamma / l}^{\text {brems }}(x)=f_{\gamma / l}^{\text {brems, }+}(x)$ $-f_{\gamma / l}^{\text {brems, }}-(x)$ is given by (Philipsen, 1992; De Florian and Frixione, 1999)

$$
\frac{d \Delta f_{\gamma / l}^{\text {brems }}}{d P^{2}}\left(x, P^{2}\right)=\frac{\alpha}{2 \pi}\left(\frac{1-(1-x)^{2}}{x} \frac{1}{P^{2}}-\frac{2 m_{l}^{2} x^{2}}{P^{4}}\right)
$$

for transverse virtual photons and by

$$
\begin{aligned}
\Delta f_{\gamma / l}^{\mathrm{brems}}(x)= & \frac{2 \lambda_{l} \alpha}{2 \pi}\left[\frac{1-(1-x)^{2}}{x} \ln \frac{Q_{\mathrm{max}}^{2}(1-x)}{m_{l}^{2} x^{2}}\right. \\
& \left.+2 m_{l}^{2} x^{2}\left(\frac{1}{Q_{\max }^{2}}-\frac{1-x}{m_{l}^{2} x^{2}}\right)\right]
\end{aligned}
$$

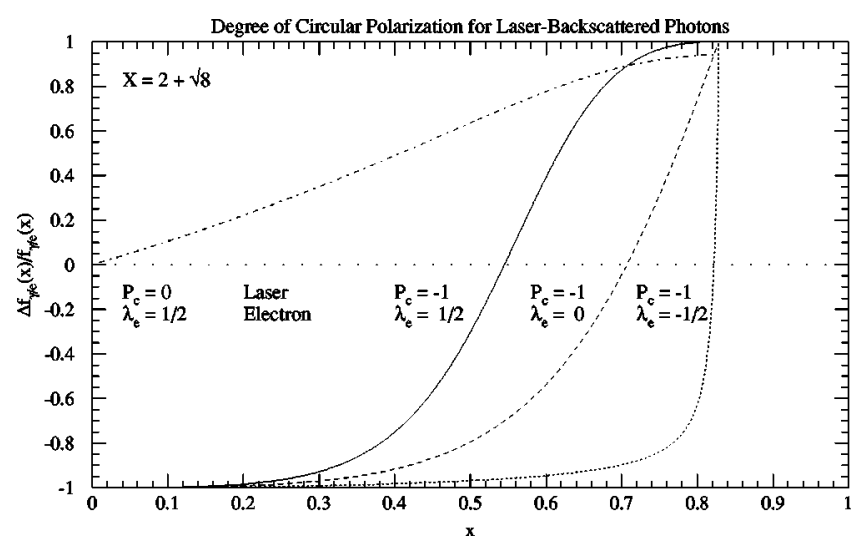

FIG. 55. Degree of circular polarization for laserbackscattered photons. The dot-dashed curve also applies to bremsstrahlung and beamstrahlung photons, but it then extends out to $x=1$ and $\Delta_{\gamma / e} f(x) / f_{\gamma / e}(x)=1$.

for almost-real photons. As in the unpolarized spectrum [Eq. (2.1)] a nonlogarithmic term is present, which is, however, not singular for $x \rightarrow 0$. At $x \simeq 1$ the photons are completely polarized parallel to the incoming lepton helicity, but at $x \simeq 0$, where most of the bremsstrahlung photons are produced, they are completely unpolarized (see dot-dashed curve in Fig. 55). The lepton polarization is lost in the Lorentz transformation from the Breit frame of the lepton-photon vertex to the center-of-mass frame of the photon-target vertex.

The circularly polarized beamstrahlung spectrum has been derived only in an integral form (Schroeder, 1990),

$$
\begin{aligned}
\Delta f_{\gamma / e}^{\text {beam }}(x)= & \frac{5 \lambda_{e}}{2 \sqrt{3} \Upsilon} \int_{u}^{\infty} d v \operatorname{Ai}(v)\left[\left(\frac{2 v}{u}-1\right)\right. \\
& \left.\times \frac{1-(1-x)^{2}}{2(1-x)}+\frac{x^{2}}{2(1-x)}\right],
\end{aligned}
$$

where the Airy function $\operatorname{Ai}(v)$ falls off exponentially at large $v$ and where $u=[5 x]^{2 / 3} /[4 \sqrt{3} Y(1-x)]^{2 / 3}$. The polarization $\Delta f_{\gamma / e}^{\text {beam }}(x) / f_{\gamma / e}^{\text {beam }}(x)$ depends only weakly on the beamstrahlung parameter $Y$ and is very similar to the bremsstrahlung polarization (Berge, Klasen, and Umeda, 2001).

While the photon polarization at an $e^{+} e^{-}$collider is thus rather limited, a photon collider offers the additional possibility of controlling the helicity of the laser photons $\left|P_{c}\right| \leqslant 1$. The outgoing photons have a polarized spectrum (Ginzburg et al., 1984)

$$
\begin{aligned}
\Delta f_{\gamma / e}^{\text {laser }}(x)= & \frac{1}{N_{c}+2 \lambda_{e} P_{c} N_{c}^{\prime}}\left\{2 \lambda_{e} \frac{x}{1-x}[1+(1-x)\right. \\
& \left.\times\left(1-\frac{2 x}{(1-x) X}\right)^{2}\right]+P_{c}\left(1-\frac{2 x}{(1-x) X}\right) \\
& \left.\times\left(1-x+\frac{1}{1-x}\right)\right\} .
\end{aligned}
$$

If only the electrons are polarized $\left(P_{c}=0\right)$, the $x$ dependence of the polarization $\Delta f_{\gamma / e}^{\text {laser }}(x) / f_{\gamma / e}^{\text {laser }}(x)$ is similar to the bremsstrahlung and beamstrahlung cases (dot- 
dashed curve in Fig. 55). However, if $P_{c}= \pm 1$, then the helicity of the backscattered photons is opposite to that of the laser photons at $x=x_{\max }$ (solid, dashed, and dotted curves in Fig. 55). Therefore the choice $2 \lambda_{e} P_{c}=-1$ guarantees not only good monochromaticity (see Fig. 1), but also a high degree of polarization of the produced photons. By switching the signs of $\lambda_{e}$ and $P_{c}$ simultaneously, one can switch the helicity of the outgoing photons without spoiling the monochromaticity of the photon spectrum.

The polarized parton densities $\Delta f_{i / \gamma}\left(Q^{2}\right)$ of the photon obey the same perturbative evolution equations (3.5) as their unpolarized counterparts, except that polarized splitting functions $\Delta P_{j \leftarrow i}$ have to be used. These have recently been calculated in next-to-leading order by Mertig and van Neerven (1996) and by Vogelsang (1996a, 1996b). We review only the leading-order results (Altarelli and Parisi, 1977),

$$
\begin{aligned}
\Delta P_{q \leftarrow q}(x)= & C_{F}\left(\frac{1+x^{2}}{(1-x)}+\frac{3}{2} \delta(1-x)\right)+\mathcal{O}\left(\alpha_{s}\right) \\
= & P_{q \leftarrow q}(x), \\
\Delta P_{g \leftarrow q}(x)= & C_{F}\left(\frac{1-(1-x)^{2}}{x}\right)+\mathcal{O}\left(\alpha_{s}\right), \\
\Delta P_{q \leftarrow g}(x)= & T_{R}\left[x^{2}-(1-x)^{2}\right]+\mathcal{O}\left(\alpha_{s}\right), \\
\Delta P_{g \leftarrow g}(x)= & N_{C}\left[\left(1+x^{4}\right)\left(\frac{1}{x}+\frac{1}{(1-x)^{+}}\right)-\frac{(1-x)^{3}}{x}\right] \\
& +\left(\frac{11}{6} N_{C}-\frac{1}{3} N_{f}\right) \delta(1-x)+\mathcal{O}\left(\alpha_{s}\right),
\end{aligned}
$$

where the Dirac matrix $\gamma_{5}$ has been assumed to anticommute with the Dirac matrices $\gamma_{\mu}$. Different results are obtained in other $\gamma_{5}$ schemes as mandated in dimensionally regularized NLO calculations. Some of them require finite renormalizations to arrive at the correct final answer. The polarized photon-quark splitting function can be obtained in leading order by the transformation $\Delta P_{q \leftarrow \gamma}=2 N_{C} e_{q}^{2} \Delta P_{q \leftarrow g}$; the polarized NLO photongluon splitting function is given by (Stratmann and Vogelsang, 1996)

$$
\begin{aligned}
\Delta P_{g \leftarrow \gamma}(x)= & \frac{\alpha_{s}\left(Q^{2}\right)}{2 \pi} N_{C} N_{f}\left\langle e^{2}\right\rangle C_{F}\left[-2(1+x) \ln ^{2} x\right. \\
& +2(x-5) \ln x-10(1-x)] .
\end{aligned}
$$

As in the unpolarized case, the pointlike solution of the polarized evolution equations dominates at large $x$ and $Q^{2}$. It takes the same functional form as Eq. (3.9), where $\Delta a_{i}$ and $\Delta b_{i}$ replace $a_{i}$ and $b_{i}$ and have been explicitly calculated in moment space in leading order (Irving and Newland, 1980; Hassan and Pilling, 1981; $\mathrm{Xu}, 1984)$ and next-to-leading order (Stratmann and Vogelsang, 1996). The position of the low- $x$ singularities in $\Delta a_{i}$ and $\Delta b_{i}$ differs from the unpolarized case, since the polarized splitting functions are now involved.
By combining the polarized parton distribution functions $\Delta f_{q / \gamma}\left(Q^{2}\right)$ and $\Delta f_{g / \gamma}\left(Q^{2}\right)$ with the appropriate Wilson coefficients (Ratcliffe, 1983; Bodwin and Qiu, 1990),

$$
\begin{aligned}
\Delta C_{q}(x)= & C_{F}\left[\left(1+x^{2}\right)\left(\frac{\ln (1-x)}{1-x}\right)_{+}\right. \\
& \left.-\frac{3}{2} \frac{1}{(1-x)_{+}}-\frac{1+x^{2}}{1-x} \ln x+2+x\right] \\
& -C_{F}\left(\frac{9}{2}+\frac{\pi^{2}}{3}\right) \delta(1-x), \\
\Delta C_{g}(x)= & T_{R}\left[(2 x-1)\left(\ln \frac{1-x}{x}-1\right)+2(1-x)\right],
\end{aligned}
$$

and (Stratmann and Vogelsang, 1996)

$$
\begin{aligned}
\Delta C_{\gamma}(x) & =2 N_{C} \Delta C_{g}(x) \\
& =3\left[(2 x-1)\left(\ln \frac{1-x}{x}-1\right)+2(1-x)\right],
\end{aligned}
$$

one obtains the NLO photon structure function in the $\overline{\mathrm{MS}}$ scheme

$$
\begin{aligned}
g_{1}^{\gamma}\left(Q^{2}\right)= & \sum_{q} e_{q}^{2}\left(\Delta f_{q / \gamma}\left(Q^{2}\right)+\frac{\alpha}{2 \pi} e_{q}^{2} \Delta C_{\gamma}+\frac{\alpha_{s}\left(Q^{2}\right)}{2 \pi}\right. \\
& \left.\times\left[\Delta C_{q} \otimes \Delta f_{q / \gamma}\left(Q^{2}\right)+\Delta C_{g} \otimes \Delta f_{g / \gamma}\left(Q^{2}\right)\right]\right) .
\end{aligned}
$$

The $\ln (1-x)$ term in the Wilson coefficient of the polarized photon causes stability problems in $g_{1}^{\gamma}$ similar to those in $F_{2}^{\gamma}$ at large $x$. Thus it should again be absorbed into the quark distributions, which also affects the polarized pointlike NLO splitting functions. The transformation is given by Eqs. (3.13) with all quantities replaced by their polarized counterparts. This DIS ${ }_{\Delta \gamma}$ factorization scheme then also leads to perturbatively stable results and to purely hadronic boundary conditions in leading and next-to-leading order.

For unpolarized photons, the hadronic input can be inferred from pionic parton densities. These are, however, not known in the polarized case. Thus Stratmann and Vogelsang (1996) were forced to make the two extreme assumptions that either $\Delta f_{i / \gamma}^{\text {had }}\left(Q_{0}^{2}\right)=f_{i / \gamma}^{\text {had }}\left(Q_{0}^{2}\right)$ or $\Delta f_{i / \gamma}^{\text {had }}\left(Q_{0}^{2}\right)=0$, as had already been suggested in the leading-order analysis of Glück, Stratmann, and Vogelsang (GStV; 1994). Current conservation implies that the first moment of $g_{1}$ vanishes (Bass, 1992; Narison, Shore, and Veneziano, 1993), which can be realized by demanding $\Delta f_{i / \gamma}^{\text {had, } n=1}=0$. This is trivial to fulfill in the second (minimal) scenario, but requires additional assumptions on the low- $x$ behavior of the first (maximal) scenario. In a previous leading-order analysis, Glück and Vogelsang (1992) considered these two scenarios only for the gluon, while they fixed $\Delta f_{q / \gamma}^{\text {had }}\left(Q_{0}^{2}\right)=f_{q / \gamma}^{\text {had }}\left(Q_{0}^{2}\right)$. The origi- 


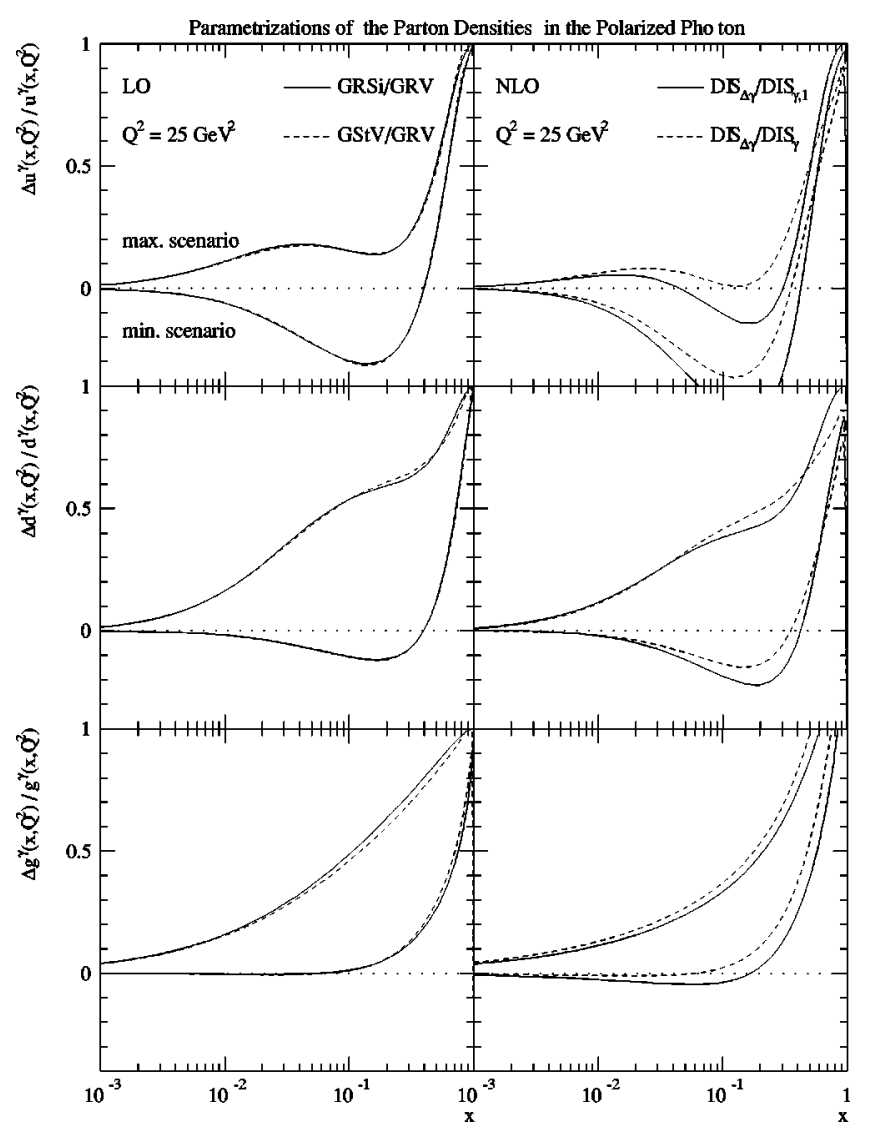

FIG. 56. Parametrizations of the polarized up-quark (top), down-quark (center), and gluon (bottom) densities in the photon in leading order (left) and next-to-leading order (right) at $Q^{2}=25 \mathrm{GeV}^{2}$.

nal DIS ${ }_{\gamma}$ scheme, which was constructed to avoid negative values of $F_{2}^{\gamma}$ at large $x$, unfortunately causes a violation of the positivity constraint $\left|g_{1}^{\gamma}\right| \leqslant F_{1}^{\gamma}=\left(F_{2}^{\gamma}\right.$ $\left.-F_{L}^{\gamma}\right) /(2 x)$. For polarized photons it is thus preferable to absorb the photonic Wilson coefficient of $F_{1}^{\gamma}, C_{1, \gamma}$ $=C_{\gamma}-4 N_{C} x(1-x)$, into the pointlike splitting functions Eqs. (3.13) [Glück, Reya, and Sieg (GRSi), 2001a]. The maximal polarized boundary conditions in the DIS $_{\Delta \gamma}$ scheme then have to be identified with the hadronic input in the DIS D $_{\gamma, 1}$ scheme, and the minimal polarized boundary conditions are given by $\Delta f_{i / \gamma}^{\text {had }}\left(Q_{0}^{2}\right) e_{q}^{2} \alpha /(2 \pi)\left(C_{1, \gamma}-C_{\gamma}\right)$. All the polarized photon densities discussed above are based on the unpolarized GRV parametrization, which imposes an incoherent VMD input at a low starting scale and is evolved in moment space with up to five massless flavors (see Sec. III.C). The different polarized parton densities in the photon are compared in Fig. 56.

Polarized photon densities can also be extended to nonzero photon virtualities, either by multiplying the boundary conditions with a suppression factor $\eta\left(P^{2}\right)$ $=\left(1+P^{2} / m_{\rho}^{2}\right)^{-2}$ (Glück, Reya, and Sieg, 2001b) to guarantee a smooth transition to real photons or by imposing the polarized virtual box result (Sasaki and Uematsu, 1999, 2000, 2001)

$$
\Delta f_{q / \gamma}^{\text {box }}\left(x, Q^{2}, P^{2}\right)=3 e_{q}^{2} \frac{\alpha}{2 \pi}(2 x-1)\left(\ln \frac{Q^{2}}{x^{2} P^{2}}-2\right)
$$

at $Q^{2}=P^{2}$. As in the unpolarized case, the virtual box can be suppressed at small $P^{2}$ by a factor $1-\eta\left(P^{2}\right)$.

The polarized cross sections for single-, two-, and three-jet photoproduction can be obtained from Eqs. (4.14), (4.21), and (4.27) by replacing the unpolarized photon spectra, parton densities, and partonic cross sections with their polarized counterparts $\Delta f_{\gamma / l}(x)$, $\Delta f_{i / \gamma}\left(x, Q^{2}\right)$, and

$$
\frac{d \Delta \sigma^{B}}{d t}=\frac{1}{2 s} \frac{1}{8 \pi s} \frac{2 g_{a, b}^{2}}{S_{a} S_{b} C_{a} C_{b}}\left(\left|\mathcal{M}_{++}^{B}\right|^{2}-\left|\mathcal{M}_{+-}^{B}\right|^{2}\right),
$$

where parity invariance guarantees that $\left|\mathcal{M}_{++}^{B}\right|^{2}$ $=\left|\mathcal{M}_{--}^{B}\right|^{2}$ and $\left|\mathcal{M}_{+-}^{B}\right|^{2}=\left|\mathcal{M}_{-+}^{B}\right|^{2}$. Similarly the polarized cross section for the photoproduction of light hadrons can be obtained from Eq. (5.14). The massless Born diagrams are the same as those in Figs. 7-9. The purely partonic (double-resolved) diagrams were evaluated by Babcock, Monsay, and Sivers (1979) using the projection operators

$$
\begin{aligned}
u(p, h) \bar{u}(p, h) & =\frac{1}{2}\left(1+h \gamma_{5}\right) p, \\
v(p, h) \bar{v}(p, h)= & \frac{1}{2}\left(1-h \gamma_{5}\right) p, \\
\epsilon^{\mu}(p, h) \epsilon^{* \nu}(p, h)= & \frac{1}{2}\left(-g^{\mu \nu}+\frac{p^{\mu} n^{\nu}+p^{\nu} n^{\mu}}{p \cdot n}\right. \\
& \left.-\frac{i h \epsilon^{\mu \nu \alpha \beta} p^{\alpha} n^{\beta}}{p \cdot n}\right)
\end{aligned}
$$

with an arbitrary four-vector $n\left(n^{2}=0\right)$ for quarks and gluons with momentum $p$ and definite helicity $h$. From their results, the direct and single-resolved matrix elements can easily be obtained by appropriate changes of couplings and color factors. They can then be applied to jet production in a polarized collider mode of HERA (Stratmann and Vogelsang, 1997) or to inclusive hadron photoproduction in fixed-target collisions (Airapetian et al., 2000; Contogouris, Grispos, and Veropoulos, 2000). Polarized NLO calculations have been performed for direct photoproduction of inclusive hadrons (De Florian and Vogelsang, 1998) and also for resolved (De Florian et al., 1999) and direct (De Florian and Frixione, 1999) photoproduction of one or two jets. If large luminosities could be accumulated in a polarized HERA or eRHIC collider, these processes could be used to determine the polarized parton densities in the photon and proton, in particular $\Delta f_{g / \gamma}$ and $\Delta f_{g / p}$. In this way the information obtained from polarized structure functions in deep-inelastic scattering would be supplemented. Figure 57 shows the pseudorapidity dependence of the single-jet asymmetry $\Delta \sigma / \sigma$ at HERA energies. The asymmetry is clearly sensitive to the polarized photon (top) and proton (bottom) densities. In addition, as a 

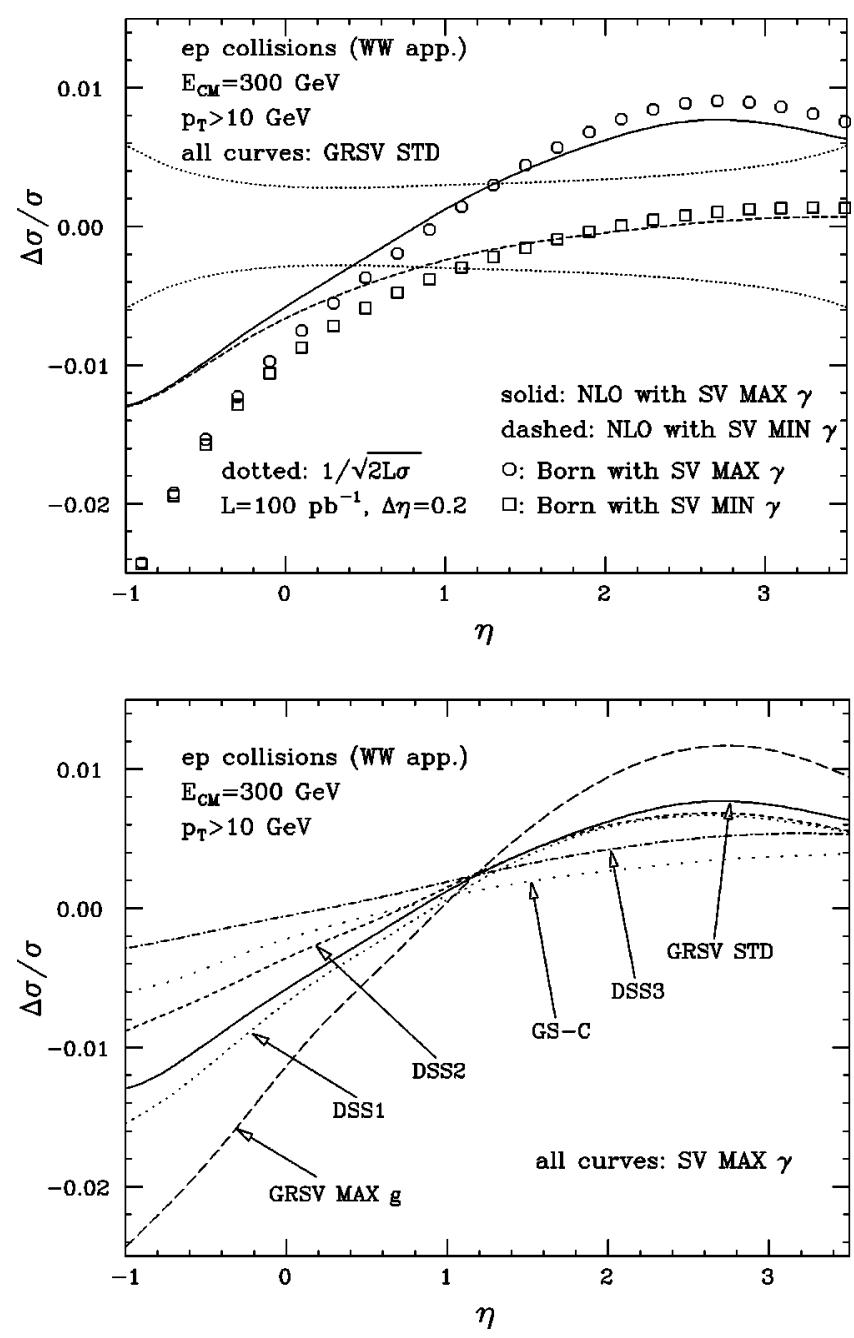

FIG. 57. Pseudorapidity dependence of the single-jet asymmetry in polarized photoproduction for different polarized photon (top) and proton (bottom) densities. From De Florian and Frixione, 1999.

ratio of cross sections it is very stable with respect to higher-order corrections and variations of the factorization and renormalization scales.

Polarized heavy-quark photoproduction proceeds through the Born diagrams shown in Figs. 31-33, which applied to the unpolarized production. Glück and Reya (1988) have calculated the photon-gluon fusion squared matrix element, which is also applicable to the photonphoton process after changing couplings and color factors. The resolved squared matrix elements have been evaluated by Contogouris, Kamal, and Papadopoulos (1990) and by Karliner and Robinett (1994), but they do not contribute significantly at the energies available at HERA and a possible GSI collider with $E_{e}=5 \mathrm{GeV}$ and $E_{p}=50 \mathrm{GeV}$ (Stratmann and Vogelsang, 1997). Due to this reduced sensitivity to the unknown polarized structure of the photon, heavy-quark photoproduction may be a useful tool to constrain the polarized gluon density in the proton, despite the fact that fragmentation effects have to be taken into account (Frixione and Ridolfi, 1996). As expected, the theoretical uncertainties are considerably reduced if NLO corrections for the direct

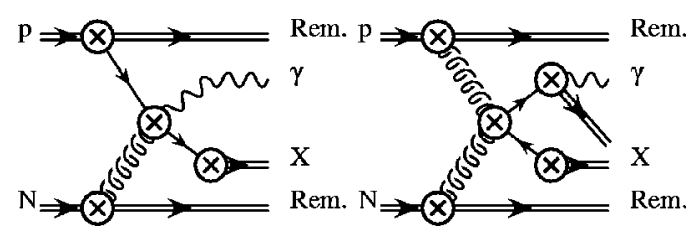

FIG. 58. Factorization of hadron-hadron scattering into photons.

(Contogouris, Kamal, and Merebashvili, 1995; Jikia and Tkabladze, 1996) and single-resolved (Bojak and Stratmann, 1998, 1999; Contogouris, Grispos, and Merebashvili, 2000a, 2000b) processes are included. For the double-resolved process the NLO corrections are not yet available.

NLO corrections are also unavailable for polarized quarkonium production in the color-singlet and coloroctet channels. However, leading-order studies suggest that polarization provides an even more stringent test of nonrelativistic QCD factorization than unpolarized quarkonium production, since the color-octet asymmetries are significantly different from the color-singlet asymmetries and since the uncertainties from the quarkonium operator values cancel in the asymmetries to a large extent. On the other hand, quarkonium production can also be used to constrain the polarized gluon density in the proton, provided that the color-octet operator values can be fixed in a different place (Chao et al., 2000; Japaridze, Nowak, and Tkabladze, 2000; Morii and Sudoh, 2000).

\section{Prompt photons in hadron collisions}

The production of prompt photons in hadron collisions is intimately related to photoproduction by crossing the initial-state photon into the final state. The contributing hadronic scattering processes are shown schematically in Fig. 58, where the photon is either produced directly in the hard partonic subprocess (left) or via fragmentation of a final-state parton (right). The inclusive cross section for the direct process Eq. (4.14) is a convolution of the parton densities in the initial hadrons, which can be pions, protons, antiprotons, or nuclei, with the partonic cross section. The fragmentation cross section Eq. (5.14) contains an additional convolution with the photon fragmentation function $D_{\gamma / c}$. The leadingorder parton diagrams and matrix elements for the fragmentation process are the same as those in Fig. 9 and Table VI, while those for the direct process are obtained by crossing the photon leg in Fig. 8 and Table VI. In these processes the photon is balanced by an outgoing jet or hadron. Double prompt-photon production can be calculated by taking into account the direct diagrams and matrix elements in Fig. 7 and Table VI and a second photon fragmentation function for the double-resolved processes discussed above.

The calculation of the NLO corrections proceeds along the lines discussed in Sec. V.B. For the inclusive direct process, they have been evaluated by Aurenche, 


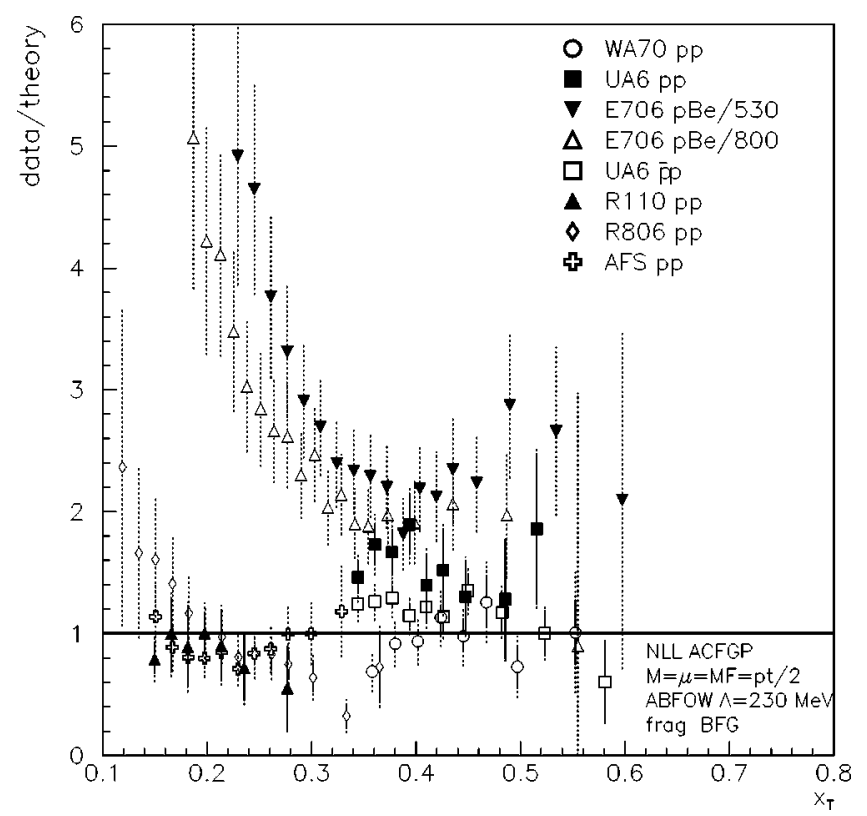

FIG. 59. Prompt-photon data from fixed-target and ISR experiments as a function of the scaled transverse momentum $x_{T}$ and normalized to next-to-leading-order QCD predictions with ABFOW (Aurenche et al., 1989) proton parton densities and BFG (Bourhis et al., 1998) photon fragmentation functions. From Aurenche, Fontannaz, et al., 1999.

Baier, et al. (1984c, 1988, 1990) for unpolarized and by Gordon and Vogelsang (1993, 1994a, 1994b) for polarized prompt-photon production. The production of a direct photon in association with an observed jet has been calculated in next-to-leading order by Baer, Ohnemus, and Owens (1990a, 1990b), by Gordon (1997a, 1997b), and by Frixione (1998b, 1999). The last two calculations have again included beam polarization. Polarized and unpolarized direct photon production with an additional final-state charm quark has been calculated in the massless scheme by Berger and Gordon $(1996,1998)$ and by Bailey, Berger, and Gordon (1996). The direct NLO corrections to double prompt-photon production have been evaluated by Aurenche, Baier, et al. (1985b), Bailey, Ohnemus, and Owens (1992), Corianò and Gordon (with polarization; 1996a, 1996b), and Binoth et al. $(2000,2001)$. NLO corrections to the fragmentation process have been evaluated by Aversa et al. (1989) and were applied to prompt-photon production by $\mathrm{Au}$ renche, Chiappetta, et al. (1993). Fragmentation typically contributes less than $20 \%$ in fixed-target collisions, but can become dominant at collider energies. An isolation cut can then help to significantly reduce the theoretical and experimental uncertainties from the fragmentation process (Berger and Qiu, 1990, 1991).

At large values of transverse momentum $p_{T}$, the leading-order QCD Compton process $q g \rightarrow q \gamma$ dominates over the competing $q \bar{q} \rightarrow g \gamma$ annihilation process. This makes prompt-photon production particularly sensitive to the gluon density in the proton, which enters only in next-to-leading order in deep-inelastic scattering. Prompt-photon production has indeed been used to constrain the unpolarized gluon density in the proton at

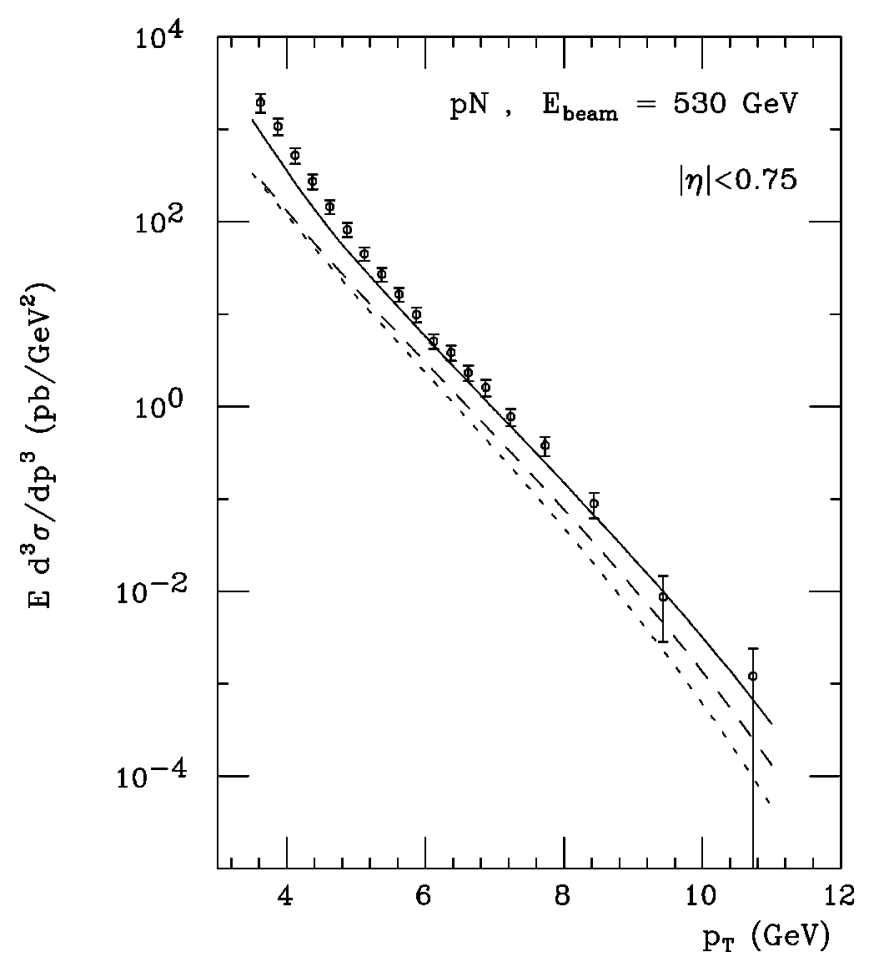

FIG. 60. Transverse momentum distribution of prompt photons produced in $p N$ collisions at $\sqrt{S}=31.5 \mathrm{GeV}$ : dotted curve, NLO; dashed curve, threshold resummed; solid curve, jointly resummed results are compared to E706 data (Laenen, Sterman, and Vogelsang, 2000).

large $x$ (Vogelsang and Vogt, 1995; Martin et al., 2000) and could also be used to constrain the polarized gluon density in the proton (Gordon and Vogelsang, 1996; Gordon, 1997c; Chang, Corianò, and Gordon, 1998). Unfortunately, these determinations suffer from poorly known fragmentation contributions, particularly of the gluon and at small $x$ (see Sec. VI.A), and potentially large logarithms in the isolation criterion (see Sec. VI.B). Even worse, the data from fixed-target and ISR experiments cannot be described consistently by NLO calculations (Aurenche, Fontannaz, et al., 1999). As Fig. 59 demonstrates, the data from the Fermilab E706 experiment in particular lie a factor of 2 or more above NLO predictions (Apanasevich et al., 1998). They can only be described if an intrinsic transverse momentum $\left\langle k_{T}\right\rangle \simeq 1.3 \mathrm{GeV}$ of the incoming partons is taken into account. This effect can be generated either by a simple Gaussian distribution or, as shown in Fig. 60, by considering multiple soft-gluon radiation and simultaneously resumming the large logarithms at small values of transverse momentum and at the partonic threshold $x_{T}$ $=2 p_{T} / \sqrt{S} \simeq 1$ (Catani, Mangano, et al., 1999; Laenen, Sterman, and Vogelsang, 2000). At collider energies the disagreement with NLO QCD is less severe and exists only at low transverse momenta, but it still leaves room for speculation on intrinsic $\left\langle k_{T}\right\rangle$ effects (Abe et al., 1994; Abbott et al., 2000b). Due to the various uncertainties discussed above, prompt-photon data have currently been dropped from global determinations of the gluon density at large $x$ (Lai et al., 2000; Martin et al., 2001). 


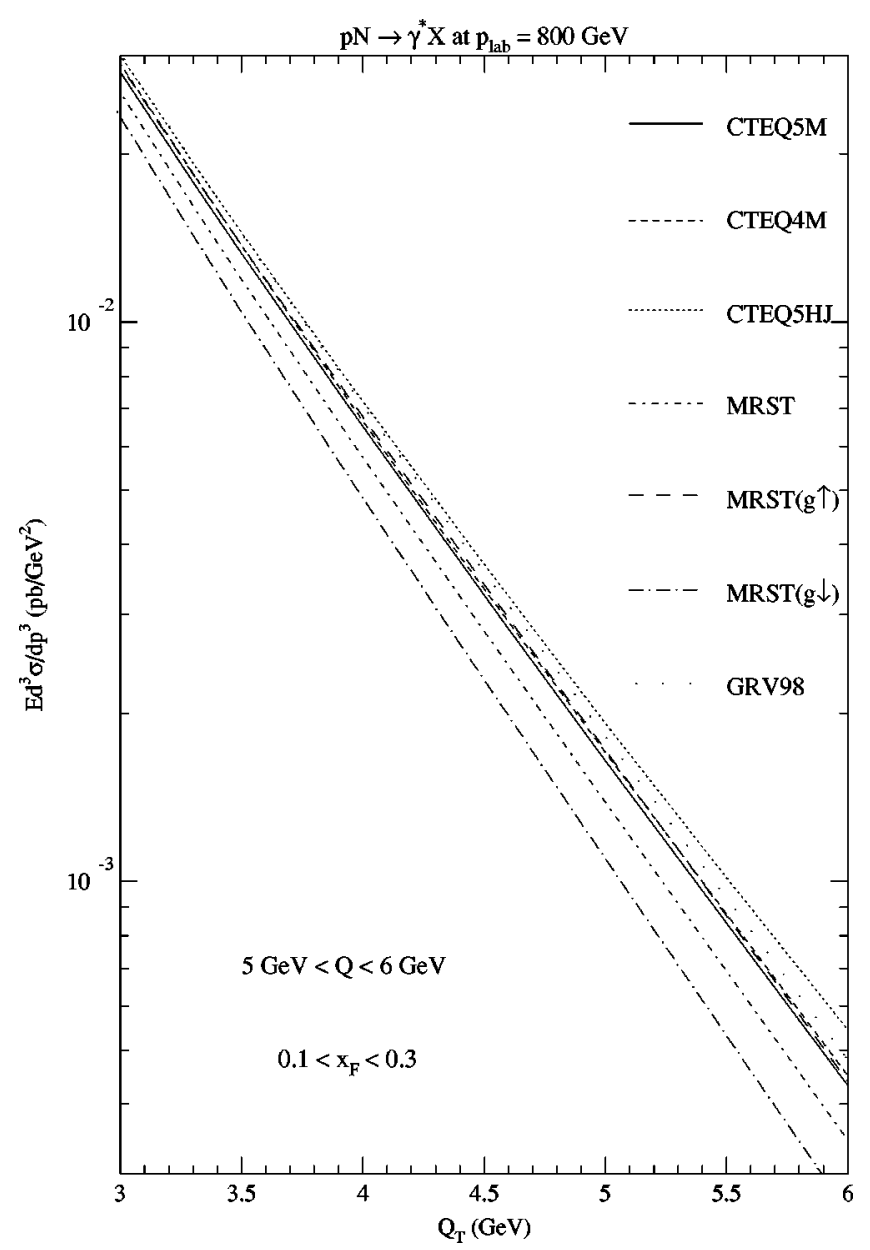

FIG. 61. Transverse momentum distribution of virtual photons produced in $p N$ collisions at $\sqrt{S}=38.8 \mathrm{GeV}$.

Instead high- $E_{T}$ jet data are used, which in turn suffer from a large factorization scheme dependence (Klasen and Kramer, 1996c; Anandam and Soper, 2000).

Like real-photon production, the production of photons with virtuality $Q$ is also dominated by the QCD Compton process at large transverse momenta $p_{T}$ $>Q / 2$ and is thus sensitive to the proton's gluon distribution (Berger, Gordon, and Klasen, 1998). However, the photon virtuality acts as a mass regulator and eliminates the fragmentation contributions. Isolation is also not necessary, since the virtual photon can be identified by its muonic decay, and the presence of the scale $Q$ may reduce the importance of large logarithms in $p_{T}$. In Fig. 61 we show that virtual-photon production in fixedtarget collisions is indeed very sensitive to the gluon density (Berger and Klasen, 2000). The polarized gluon distribution could be determined in a similar way (Berger, Gordon, and Klasen, 2000a, 2000b).

\section{SUMMARY}

From the fixed-target experiments in the 1980s to modern $e^{+} e^{-}$and $e p$ colliders, hard photoproduction has been a fertile research ground experimentally and theoretically. Precise measurements and calculations at next-to-leading order of perturbative QCD have been performed for a large variety of processes, and they have served a multitude of purposes. For one thing, the factorization theorem for hard scattering processes has been thoroughly tested. The photon energy spectra have been improved to meet the precision mandated by current and future lepton colliders. The parton densities of the photon, proton, and pion, and in particular the gluon densities are now better constrained, which reduces the theoretical uncertainty at hadron and photon colliders. Ambiguities associated with jet algorithms and kinematic regions of large hadronization corrections have been identified and eliminated. Perturbative and nonperturbative aspects of parton fragmentation into individual hadrons and photons and of quarkonium formation have been disentangled, and the universality of fitted fragmentation functions and quarkonium operator expectation values has been tested.

In spite of all these successes, research into hard photoproduction is far from complete: There is still much room for improvements, not only in even more precise next-to-next-to-leading-order calculations, but also in determinations of the gluon density and spin structure of photons and protons, of the transition from real to virtual photons, and of photon interactions with new hypothetical particles as predicted, for example, by supersymmetry. Exciting possibilities may soon open up with a new linear $e^{+} e^{-}$collider, which may even include a dedicated photon-photon experiment with backscattered laser beams. Such an experiment would certainly initiate a whole new era of hard photoproduction.

\section{ACKNOWLEDGMENTS}

I wish to thank I. Schienbein and C. Sieg for providing me with the virtual and polarized parton distributions in the photon, B. A. Kniehl and G. Kramer for carefully reading and commenting on the manuscript, and my family for their strong support. Financial support by the Deutsche Forschungsgemeinschaft through Grant Nos. KL 1266/1-1 and KL 1266/1-2 and by the European Commission through Grant No. ERBFMRX-CT98-0194 is gratefully acknowledged.

\section{REFERENCES}

Abbiendi, G., et al. (OPAL Collaboration), 1999, Eur. Phys. J. C 10, 547.

Abbiendi, G., et al. (OPAL Collaboration), 2000, Eur. Phys. J. C 16, 579.

Abbiendi, G., et al. (OPAL Collaboration), 2001a, in International Europhysics Conference on High Energy Physics 2001, Budapest, Hungary (in press).

Abbiendi, G., et al. (OPAL Collaboration), 2001b, in International Europhysics Conference on High Energy Physics 2001, Budapest, Hungary (in press).

Abbiendi, G., et al. (OPAL Collaboration), 2001c, in International Europhysics Conference on High Energy Physics 2001, Budapest, Hungary (in press). 
Abbiendi, G., et al. (OPAL Collaboration), 2001d, OPAL physics note PN492.

Abbiendi, G., I. G. Knowles, G. Marchesini, M. H. Seymour, L. Stanco, and B. R. Webber, 1992, Comput. Phys. Commun. 67, 465 .

Abbott, B., et al. (D0 Collaboration), 2000a, Phys. Lett. B 487, 264.

Abbott, B., et al. (D0 Collaboration), 2000b, Phys. Rev. Lett. 84, 2786.

Abbott, B., et al. (D0 Collaboration), 2000c, Phys. Rev. Lett. 85, 5068.

Abe, F., et al. (CDF Collaboration), 1992, Phys. Rev. Lett. 69, 3704.

Abe, F., et al. (CDF Collaboration), 1993, Phys. Rev. Lett. 71, 500.

Abe, F., et al. (CDF Collaboration), 1994, Phys. Rev. Lett. 73, 2662; 74, 1891(E).

Abe, F., et al. (CDF Collaboration), 1995, Phys. Rev. Lett. 75, 1451.

Abe, F., et al. (CDF Collaboration), 1997, Phys. Rev. Lett. 79, 572.

Abramowicz, H., and A. Caldwell, 1999, Rev. Mod. Phys. 71, 1275.

Abramowicz, H., K. Charchula, and A. Levy, 1991, Phys. Lett. B 269, 458.

Abramowicz, H., E. Gurvich, and A. Levy, 1998, Phys. Lett. B 420, 104.

Abreu, P., et al. (DELPHI Collaboration), 1995, Z. Phys. C 69, 1.

Abt, I., et al. (H1 Collaboration), 1993, Phys. Lett. B 314, 436. Abt, I., et al. (H1 Collaboration), 1994, Phys. Lett. B 328, 176. Acciarri, M., et al. (L3 Collaboration), 1999a, Phys. Lett. B 453, 83.

Acciarri, M., et al. (L3 Collaboration), 1999b, Phys. Lett. B 467, 137.

Acciarri, M., et al. (L3 Collaboration), 2000, Phys. Lett. B 483, 373.

Acciarri, M., et al. (L3 Collaboration), 2001a, Phys. Lett. B $\mathbf{5 0 3}, 10$.

Acciarri, M., et al. (L3 Collaboration), 2001b, Phys. Lett. B 514, 19.

Achard, P., et al. (L3 Collaboration), 2001, in International Europhysics Conference on High Energy Physics 2001, Budapest, Hungary, preprint hep-ex/0109037.

Ackerstaff, K., et al. (OPAL Collaboration), 1997, Z. Phys. C 73, 433.

Ackerstaff, K., et al. (OPAL Collaboration), 1998, Eur. Phys. J. C 2, 39.

Ackerstaff, K., et al. (OPAL Collaboration), 1999, Eur. Phys. J. C 6, 253.

Acton, P. D., et al. (OPAL Collaboration), 1993, Z. Phys. C 58, 405.

Adachi, K., et al. (TOPAZ Collaboration), 1999, Phys. Lett. B 451, 256.

Adamovich, M. I., et al. (Photon Emulsion Collaboration), 1980, Phys. Lett. 89B, 427.

Adamovich, M. I., et al. (Photon Emulsion Collaboration), 1987, Phys. Lett. B 187, 437.

Adams, D., et al. (E683 Collaboration), 1994, Phys. Rev. Lett. 72, 2337.

Adloff, C., et al. (H1 Collaboration), 1997, Z. Phys. C 76, 213. Adloff, C., et al. (H1 Collaboration), 1998, Eur. Phys. J. C 1, 97.
Adloff, C., et al. (H1 Collaboration), 1999a, Eur. Phys. J. C 10, 363.

Adloff, C., et al. (H1 Collaboration), 1999b, Phys. Lett. B 467, 156; 518, 331(E).

Adloff, C., et al. (H1 Collaboration), 1999c, Nucl. Phys. B 538, 3.

Adloff, C., et al. (H1 Collaboration), 2000, Phys. Lett. B 483, 36.

Adloff, C., et al. (H1 Collaboration), 2001a, in International Europhysics Conference on High Energy Physics 2001, Budapest, Hungary (in press).

Adloff, C., et al. (H1 Collaboration), 2001b, in International Europhysics Conference on High Energy Physics 2001, Budapest, Hungary (in press).

Adloff, C., et al. (H1 Collaboration), 2001c, in International Europhysics Conference on High Energy Physics 2001, Budapest, Hungary (in press).

Adolphsen, C., et al. (Internationàl Study Group Collaboration), 2000, SLAC-R-559.

Adriani, O., et al. (L3 Collaboration), 1992, Phys. Lett. B 292, 472.

Adriani, O., et al. (L3 Collaboration), 1993, Phys. Lett. B 301, 136.

Ahmed, T., et al. (H1 Collaboration), 1995, Nucl. Phys. B 445, 195.

Aid, S., et al. (H1 Collaboration), 1996a, Z. Phys. C 70, 17.

Aid, S., et al. (H1 Collaboration), 1996b, Nucl. Phys. B 472, 32.

Airapetian, A., et al. (HERMES Collaboration), 2000, Phys. Rev. Lett. 84, 2584.

Akers, R., et al. (OPAL Collaboration), 1994, Z. Phys. C 63, 197.

Akrawy, M. Z., et al. (OPAL Collaboration), 1990, Phys. Lett. B 246, 285.

Albino, S., M. Klasen, and S. Söldner-Rembold, 2002, DESY02-052. Also available as e-print hep-ph/0205069.

Alexander, G., et al. (OPAL Collaboration), 1991, Phys. Lett. B 264, 219.

Ali, A., J. G. Körner, G. Kramer, and J. Willrodt, 1980, Nucl. Phys. B 168, 409.

Ali, A., G. Kramer, E. Pietarinen, and J. Willrodt, 1980, Phys. Lett. 93B, 155.

Alston-Garnjost, M., et al. (TPC/2 $\gamma$ Collaboration), 1990, Phys. Lett. B 252, 499.

Altarelli, G., R. K. Ellis, G. Martinelli, and S. Pi, 1979, Nucl. Phys. B 160, 301.

Altarelli, G., and G. Parisi, 1977, Nucl. Phys. B 126, 298.

Althoff, M., et al. (TASSO Collaboration), 1984, Phys. Lett. 138B, 219.

Alvarez, M. P., et al. (NA14/2 Collaboration), 1992, Phys. Lett. B 278, 385.

Alvarez, M. P., et al. (NA14/2 Collaboration), 1993, Z. Phys. C 60, 53.

Amaldi, U., et al. 1979, in Proceedings of the Study of an EP Facility for Europe, Hamburg, Germany, edited by U. Amaldi (Deutschos Elektronen-Synchrotron, Hamburg), p. 377.

Amati, D., R. Petronzio, and G. Veneziano, 1978a, Nucl. Phys. B 140, 54.

Amati, D., R. Petronzio, and G. Veneziano, 1978b, Nucl. Phys. B 146, 29.

Amundson, J. F., O. J. Éboli, E. M. Gregores, and F. Halzen, 1997, Phys. Lett. B 390, 323.

Amundson, J. F., S. Fleming, and I. Maksymyk, 1997, Phys. Rev. D 56, 5844. 
Anandam, P., and D. E. Soper, 2000, Phys. Rev. D 61, 094003. Andersson, B., G. Gustafson, G. Ingelman, and T. Sjöstrand, 1983, Phys. Rep. 97, 31.

Anjos, J. C., et al. (E691 Collaboration), 1989, Phys. Rev. Lett. 62, 513.

Anjos, J. C., et al. (E691 Collaboration), 1990, Phys. Rev. Lett. 65, 2503.

Anselmino, M., P. Kroll, and E. Leader, 1983, Z. Phys. C 18, 307.

Antoniadis, I., and G. Grunberg, 1983, Nucl. Phys. B 213, 445. Apanasevich, L., et al. (Fermilab E706 Collaboration), 1998, Phys. Rev. Lett. 81, 2642.

Aso, T., et al. (AMY Collaboration), 1995, Phys. Lett. B 363, 249.

Auge, E., et al. (NA14 Collaboration), 1986, Phys. Lett. 168B, 163.

Aurenche, P., R. Baier, A. Douiri, M. Fontannaz, and D. Schiff, 1984a, Z. Phys. C 24, 309.

Aurenche, P., R. Baier, A. Douiri, M. Fontannaz, and D. Schiff, 1984b, Phys. Lett. 135B, 164.

Aurenche, P., R. Baier, A. Douiri, M. Fontannaz, and D. Schiff, 1984c, Phys. Lett. 140B, 87.

Aurenche, P., R. Baier, A. Douiri, M. Fontannaz, and D. Schiff, 1985a, Z. Phys. C 29, 423.

Aurenche, P., R. Baier, A. Douiri, M. Fontannaz, and D. Schiff, 1985b, Z. Phys. C 29, 459.

Aurenche, P., R. Baier, A. Douiri, M. Fontannaz, and D. Schiff, 1987, Nucl. Phys. B 286, 553.

Aurenche, P., R. Baier, and M. Fontannaz, 1990, Phys. Rev. D 42, 1440.

Aurenche, P., R. Baier, M. Fontannaz, J. F. Owens, and M. Werlen, 1989, Phys. Rev. D 39, 3275.

Aurenche, P., R. Baier, M. Fontannaz, and D. Schiff, 1988, Nucl. Phys. B 297, 661.

Aurenche, P., L. Bourhis, M. Fontannaz, and J. P. Guillet, 2000, Eur. Phys. J. C 17, 413.

Aurenche, P., P. Chiappetta, M. Fontannaz, J. P. Guillet, and E. Pilon, 1992, Z. Phys. C 56, 589.

Aurenche, P., P. Chiappetta, M. Fontannaz, J. P. Guillet, and E. Pilon, 1993, Nucl. Phys. B 399, 34.

Aurenche, P., M. Fontannaz, J. Fujimoto, J. P. Guillet, K. Kato, and Y. Shimizu, 1994a, Prog. Theor. Phys. 92, 175.

Aurenche, P., M. Fontannaz, J. Fujimoto, J. P. Guillet, K. Kato, and Y. Shimizu, 1994b, in Workshop on Two-Photon Physics at LEP and HERA, Proceedings, edited by G. Jarlskog and L. Jonsson (Lund University, Lund), p. 269. Also available as e-print hep-ph/9409294.

Aurenche, P., M. Fontannaz, and J. P. Guillet, 1994a, Z. Phys. C 64, 621.

Aurenche, P., M. Fontannaz, and J. P. Guillet, 1994b, Phys. Lett. B 338, 98.

Aurenche, P., M. Fontannaz, J. P. Guillet, B. Kniehl, E. Pilon, and M. Werlen, 1999, Eur. Phys. J. C 9, 107.

Aurenche, P., M. Fontannaz, J. P. Guillet, A. Kotikov, and E. Pilon, 1997, Phys. Rev. D 55, 1124.

Aversa, F., P. Chiappetta, L. Gonzales, M. Greco, and J. P. Guillet, 1991, Z. Phys. C 49, 459.

Aversa, F., P. Chiappetta, M. Greco, and J. P. Guillet, 1989, Nucl. Phys. B 327, 105.

Aversa, F., P. Chiappetta, M. Greco, and J. P. Guillet, 1990, Z. Phys. C 46, 253.

Babcock, J., E. Monsay, and D. Sivers, 1979, Phys. Rev. D 19, 1483.
Baer, H., J. Ohnemus, and J. F. Owens, 1989a, Z. Phys. C 42, 657.

Baer, H., J. Ohnemus, and J. F. Owens, 1989b, Phys. Rev. D 40, 2844.

Baer, H., J. Ohnemus, and J. F. Owens, 1990a, Phys. Lett. B 234, 127.

Baer, H., J. Ohnemus, and J. F. Owens, 1990b, Phys. Rev. D 42 , 61.

Baier, R., J. Engels, and B. Petersson, 1979, Z. Phys. C 2, 265.

Baier, R., and K. Fey, 1979, Z. Phys. C 2, 339.

Baier, R., and R. Rückl, 1981, Phys. Lett. 102B, 364.

Bailey, B., E. L. Berger, and L. E. Gordon, 1996, Phys. Rev. D 54, 1896.

Bailey, B., J. Ohnemus, and J. F. Owens, 1992, Phys. Rev. D 46, 2018.

Barate, R., et al. (NA14 Collaboration), 1986, Phys. Lett. B 174, 458.

Barate, R., et al. (ALEPH Collaboration), 2000, in ICHEP 2000, 30th International Conference on High Energy Physics, Osaka, Japan, edited by C. S. Lim and T. Yamanaka (World Scientific, Singapore), p. 411.

Bardeen, W. A., and A. J. Buras, 1979, Phys. Rev. D 20, 166; 21, 2041(E).

Bardeen, W. A., A. J. Buras, D. W. Duke, and T. Muta, 1978, Phys. Rev. D 18, 3998.

Bartel, W., et al. (JADE Collaboration), 1980, Phys. Lett. 91B, 142.

Bartel, W., et al. (JADE Collaboration), 1981, Phys. Lett. 107B, 163.

Bartel, W., et al. (JADE Collaboration), 1987, Phys. Lett. B 184, 288.

Bass, S. D., 1992, Int. J. Mod. Phys. A 7, 6039.

Bauer, T. H., F. M. Pipkin, R. D. Spital, and D. R. Yennie, 1978, Rev. Mod. Phys. 50, 261.

Bawa, A. C., M. Krawczyk, and W. J. Stirling, 1991, Z. Phys. C 50, 293.

Beenakker, W., H. Kuijf, W. L. van Neerven, and J. Smith, 1989, Phys. Rev. D 40, 54.

Beenakker, W., W. L. van Neerven, R. Meng, G. A. Schuler, and J. Smith, 1991, Nucl. Phys. B 351, 507.

Behrend, H. J., et al. (CELLO Collaboration), 1982, Phys. Lett. 110B, 329.

Behrend, H. J., et al. (CELLO Collaboration), 1991, Z. Phys. C 51, 365.

Beneke, M., M. Krämer, and M. Vänttinen, 1998, Phys. Rev. D 57, 4258.

Beneke, M., I. Z. Rothstein, and M. B. Wise, 1997, Phys. Lett. B 408, 373.

Beneke, M., G. A. Schuler, and S. Wolf, 2000, Phys. Rev. D 62 , 034004.

Berezhnoy, A. V., V. V. Kiselev, and A. K. Likhoded, 2000, Phys. Rev. D 62, 074013.

Berezhnoy, A. V., and A. K. Likhoded, 2001, Yad. Fiz. 64, 1913 [Phys. At. Nucl. 64, 1830 (2001)].

Berge, S., M. Klasen, and Y. Umeda, 2001, Phys. Rev. D 63, 035003 .

Berger, C., et al. (PLUTO Collaboration), 1978, Phys. Lett. 78B, 176.

Berger, C., et al. (PLUTO Collaboration), 1979, Phys. Lett. 89B, 120.

Berger, C., et al. (PLUTO Collaboration), 1980, Phys. Lett. 97B, 459. 
Berger, C., et al. (PLUTO Collaboration), 1984a, Z. Phys. C 26, 191.

Berger, C., et al. (PLUTO Collaboration), 1984b, Phys. Lett. 142B, 119.

Berger, C., et al. (PLUTO Collaboration), 1985, Z. Phys. C 29, 499.

Berger, C., et al. (PLUTO Collaboration), 1987, Z. Phys. C 33, 351.

Berger, E. L., and L. E. Gordon, 1996, Phys. Rev. D 54, 2279. Berger, E. L., and L. E. Gordon, 1998, Phys. Rev. D 58, 114024. Berger, E. L., L. E. Gordon, and M. Klasen, 1998, Phys. Rev. D 58, 074012.

Berger, E. L., L. E. Gordon, and M. Klasen, 2000a, Phys. Rev. D 62, 014014.

Berger, E. L., L. E. Gordon, and M. Klasen, 2000b, RIKEN Rev. 28, 44.

Berger, E. L., X. Guo, and J. Qiu, 1996, Phys. Rev. Lett. 76, 2234.

Berger, E. L., and D. Jones, 1981, Phys. Rev. D 23, 1521.

Berger, E. L., and M. Klasen, 2000, Nucl. Phys. B, Proc. Suppl. 82, 179.

Berger, E. L., and J. Qiu, 1990, Phys. Lett. B 248, 371.

Berger, E. L., and J. Qiu, 1991, Phys. Rev. D 44, 2002.

Bernreuther, W., J. P. Ma, and B. H. McKellar, 1995, Phys. Rev.

D 51, 2475.

Bethke, S., et al. (JADE Collaboration), 1988, Phys. Lett. B 213, 235.

Binnewies, J., 1997, Ph.D. thesis (Hamburg University), DESY-97-128. Available as e-print hep-ph/9707269.

Binnewies, J., B. A. Kniehl, and G. Kramer, 1995a, Z. Phys. C 65, 471.

Binnewies, J., B. A. Kniehl, and G. Kramer, 1995b, Phys. Rev. D 52, 4947.

Binnewies, J., B. A. Kniehl, and G. Kramer, 1996a, Phys. Rev. D 53, 3573.

Binnewies, J., B. A. Kniehl, and G. Kramer, 1996b, Phys. Rev. D 53, 6110.

Binnewies, J., B. A. Kniehl, and G. Kramer, 1997, Z. Phys. C 76, 677.

Binnewies, J., B. A. Kniehl, and G. Kramer, 1998a, Phys. Rev. D 58, 014014.

Binnewies, J., B. A. Kniehl, and G. Kramer, 1998b, Phys. Rev. D 58, 034016.

Binoth, T., J. P. Guillet, E. Pilon, and M. Werlen, 2000, Eur. Phys. J. C 16, 311.

Binoth, T., J. P. Guillet, E. Pilon, and M. Werlen, 2001, Phys. Rev. D 63, 114016

Bjorken, J. D., 1978, Phys. Rev. D 17, 171.

Blair, R., G. Grindhammer, M. Klasen, and M. Krämer, 2000, in 8th International Workshop on Deep Inelastic Scattering (DIS-2000), Bologna, Italy, edited by J. A. Gracey and T. Greenshaw (World Scientific, Singapore), p. 668.

Bloch, F., and A. Nordsieck, 1937, Phys. Rev. 52, 54.

Bodwin, G. T., E. Braaten, and G. P. Lepage, 1995, Phys. Rev. D 51, 1125; 55, 5853(E).

Bodwin, G. T., and B. W. Harris, 2001, Phys. Rev. D 63, 077503.

Bodwin, G. T., and J. Qiu, 1990, Phys. Rev. D 41, 2755.

Bödeker, D., 1992a, Phys. Lett. B 292, 164.

Bödeker, D., 1992b, Ph.D. thesis (Hamburg University).

Bödeker, D., 1993, Z. Phys. C 59, 501.

Bödeker, D., G. Kramer, and S. G. Salesch, 1994, Z. Phys. C 63, 471 .
Bojak, I., and M. Stratmann, 1998, Phys. Lett. B 433, 411.

Bojak, I., and M. Stratmann, 1999, Nucl. Phys. B 540, 345.

Bollini, C. G., and J. J. Giambiagi, 1972a, Phys. Lett. 40B, 566.

Bollini, C. G., and J. J. Giambiagi, 1972b, Nuovo Cimento Soc. Ital. Fis., B 12, 20.

Borzumati, F. M., B. A. Kniehl, and G. Kramer, 1993, Z. Phys. C 59, 341.

Borzumati, F. M., and G. A. Schuler, 1993, Z. Phys. C 58, 139. Bourhis, L., M. Fontannaz, and J. P. Guillet, 1998, Eur. Phys. J. C 2, 529 .

Bourhis, L., M. Fontannaz, J. P. Guillet, and M. Werlen, 2001, Eur. Phys. J. C 19, 89.

Bowler, M. G., 1981, Z. Phys. C 11, 169.

Braaten, E., K. Cheung, S. Fleming, and T. C. Yuan, 1995, Phys. Rev. D 51, 4819.

Braaten, E., K. Cheung, and T. C. Yuan, 1993a, Phys. Rev. D 48, R5049.

Braaten, E., K. Cheung, and T. C. Yuan, 1993b, Phys. Rev. D 48, 4230.

Braaten, E., and S. Fleming, 1995, Phys. Rev. Lett. 74, 3327.

Braaten, E., S. Fleming, and T. C. Yuan, 1996, Annu. Rev. Nucl. Part. Sci. 46, 197.

Braaten, E., Y. Jia, and T. Mehen, 2002, Phys. Rev. D 66, 014003.

Braaten, E., B. A. Kniehl, and J. Lee, 2000, Phys. Rev. D 62 , 094005

Braaten, E., and J. Lee, 2000, Nucl. Phys. B 586, 427.

Braaten, E., and J. Lee, 2002, Phys. Rev. D 65, 034005.

Braaten, E., and T. C. Yuan, 1993, Phys. Rev. Lett. 71, 1673.

Braaten, E., and T. C. Yuan, 1994, Phys. Rev. D 50, 3176.

Braaten, E., and T. C. Yuan, 1995, Phys. Rev. D 52, 6627.

Brandelik, R., et al. (TASSO Collaboration), 1980, Phys. Lett. 97B, 453.

Brandelik, R., et al. (TASSO Collaboration), 1981, Phys. Lett. 107B, 290.

Braunschweig, W., et al. (TASSO Collaboration), 1990, Z. Phys. C 47, 499.

Breitweg, J., et al. (ZEUS Collaboration), 1997, Phys. Lett. B 401, 192.

Breitweg, J., et al. (ZEUS Collaboration), 1998a, Eur. Phys. J. C 4, 591.

Breitweg, J., et al. (ZEUS Collaboration), 1998b, in ICHEP 1998, 29th International Conference on High-Energy Physics, Vancouver, Canada, edited by A. Astbury, D. Axen, and J. Robinson (World Scientific, Singapore), p. 765.

Breitweg, J., et al. (ZEUS Collaboration), 1998c, Eur. Phys. J. C 1, 109.

Breitweg, J., et al. (ZEUS Collaboration), 1998d, Eur. Phys. J. C 2, 61.

Breitweg, J., et al. (ZEUS Collaboration), 1998e, Phys. Lett. B 443, 394.

Breitweg, J., et al. (ZEUS Collaboration), 1998f, Eur. Phys. J. C 2, 77.

Breitweg, J., et al. (ZEUS Collaboration), 1999a, Eur. Phys. J. C 6, 67.

Breitweg, J., et al. (ZEUS Collaboration), 1999b, Eur. Phys. J. C 6, 239.

Breitweg, J., et al. (ZEUS Collaboration), 1999c, Eur. Phys. J. C 11, 35.

Breitweg, J., et al. (ZEUS Collaboration), 2000a, in ICHEP 2000, 30th International Conference on High-Energy Physics, Osaka, Japan, edited by C. S. Lim and T. Yamanaka (World Scientific, Singapore), p. 494. 
Breitweg, J., et al. (ZEUS Collaboration), 2000b, in ICHEP 2000, 30th International Conference on High-Energy Physics, Osaka, Japan, edited by C. S. Lim and T. Yamanaka (World Scientific, Singapore), p. 408.

Breitweg, J., et al. (ZEUS Collaboration), 2000c, DESY-00142. Also available as e-print hep-ex/0010019.

Breitweg, J., et al. (ZEUS Collaboration), 2000d, Phys. Lett. B 472, 175.

Breitweg, J., et al. (ZEUS Collaboration), 2000e, Phys. Lett. B 481, 213.

Breitweg, J., et al. (ZEUS Collaboration), 2001, Eur. Phys. J. C 18, 625 .

Brinkmann, R., K. Flöttmann, J. Rossbach, P. Schmüser, N. Walker, and H. Weise, 2001, "TESLA Technical Design Report, Part 2: The Accelerator," DESY-01-011B.

Brodsky, S. J., T. A. DeGrand, J. F. Gunion, and J. H. Weis, 1978, Phys. Rev. Lett. 41, 672.

Brodsky, S. J., T. A. DeGrand, J. F. Gunion, and J. H. Weis, 1979, Phys. Rev. D 19, 1418.

Brugnera, R., 2001, in Deep Inelastic Scattering (DIS-2001), Bologna, Italy (in press).

Budnev, V. M., I. F. Ginzburg, G. V. Meledin, and V. G. Serbo, 1974, Phys. Rep. 15, 181.

Buskulic, D., et al. (ALEPH Collaboration) 1993, Z. Phys. C 57, 17.

Buskulic, D., et al. (ALEPH Collaboration), 1995, Phys. Lett. B 355, 595.

Buskulic, D., et al. (ALEPH Collaboration), 1996, Z. Phys. C 69, 365 .

Butterworth, J. M., L. Feld, M. Klasen, and G. Kramer, 1996, in Future Physics at HERA: Proceedings, 2 vols., edited by G. Ingelman, A. De Roeck, and R. Klanner, DESY-96-235, p. 554. Also available as e-print hep-ph/9608481.

Cacciari, M., S. Frixione, and P. Nason, 2001, J. High Energy Phys. 0103, 006.

Cacciari, M., and M. Greco, 1996, Z. Phys. C 69, 459.

Cacciari, M., and M. Greco, 1997, Phys. Rev. D 55, 7134.

Cacciari, M., M. Greco, B. A. Kniehl, M. Krämer, G. Kramer, and M. Spira, 1996, Nucl. Phys. B 466, 173.

Cacciari, M., M. Greco, and M. Krämer, 1997, Phys. Rev. D 55, 7126.

Cacciari, M., M. Greco, S. Rolli, and A. Tanzini, 1997, Phys. Rev. D 55, 2736.

Cacciari, M., and M. Krämer, 1996, Phys. Rev. Lett. 76, 4128.

Catani, S., Y. L. Dokshitzer, M. Olsson, G. Turnock, and B. R. Webber, 1991, Phys. Lett. B 269, 432.

Catani, S., Y. L. Dokshitzer, M. H. Seymour, and B. R. Webber, 1993, Nucl. Phys. B 406, 187.

Catani, S., Y. L. Dokshitzer, and B. R. Webber, 1992, Phys. Lett. B 285, 291.

Catani, S., M. Fontannaz, and E. Pilon, 1998, Phys. Rev. D 58, 094025.

Catani, S., M. L. Mangano, P. Nason, C. Oleari, and W. Vogelsang, 1999, J. High Energy Phys. 9903, 025.

Catani, S., and M. H. Seymour, 1996, Phys. Lett. B 378, 287.

Catani, S., and M. H. Seymour, 1997, Nucl. Phys. B 485, 291; 510, 503(E).

Chang, S., C. Corianò, and L. E. Gordon, 1998, Phys. Rev. D 58, 074002 .

Chao, K., H. Dong, L. Hao, and F. Yuan, 2000, Phys. Rev. D 61, 114013.

Chao, K., L. Hao, and F. Yuan, 1999, Phys. Rev. Lett. 83, 4490.
Chapkin, M., 2002, in Proceedings of the 7th International Workshop on Meson Production, Properties and Interaction, Krakow, Poland (in press).

Chekanov, S., et al. (ZEUS Collaboration), 2001, Phys. Lett. B 511, 19.

Chen, P., 1992, Phys. Rev. D 46, 1186.

Chiappetta, P., M. Greco, J. P. Guillet, S. Rolli, and M. Werlen, 1994, Nucl. Phys. B 412, 3.

Cho, P., and A. K. Leibovich, 1996a, Phys. Rev. D 53, 150.

Cho, P., and A. K. Leibovich, 1996b, Phys. Rev. D 53, 6203.

Choi, S. Y., and K. Hagiwara, 1995, Phys. Lett. B 359, 369.

Chýla, J., and M. Taševský, 2001, Eur. Phys. J. C 18, 723.

Collins, J. C., D. E. Soper, and G. Sterman, 1988, Adv. Ser. Dir.

High Energy Phys. 5, 1.

Collins, P. D., and T. P. Spiller, 1985, J. Phys. G 11, 1289.

Contogouris, A. P., G. Grispos, and Z. Merebashvili, 2000a, Phys. Lett. B 482, 93.

Contogouris, A. P., G. Grispos, and Z. Merebashvili, 2000b, Phys. Rev. D 62, 114509.

Contogouris, A. P., G. Grispos, and G. Veropoulos, 2000, Phys. Rev. D 62, 014023.

Contogouris, A. P., B. Kamal, and Z. Merebashvili, 1995, Phys. Rev. D 51, 4808; 55, 3229(E).

Contogouris, A. P., B. Kamal, and S. Papadopoulos, 1990, Phys. Lett. B 246, 523.

Cords, D., et al. 1993, Phys. Lett. B 302, 341.

Corianò, C., and L. E. Gordon, 1996a, Nucl. Phys. B 469, 202.

Corianò, C., and L. E. Gordon, 1996b, Phys. Rev. D 54, 781.

Curci, G., W. Furmanski, and R. Petronzio, 1980, Nucl. Phys. B 175, 27.

Curtis, R. B., 1956, Phys. Rev. 104, 211.

D'Alesio, U., and H. J. Pirner, 2000, Eur. Phys. J. A 7, 109.

Dalitz, R. H., and D. R. Yennie, 1957, Phys. Rev. 105, 1598.

Da Luz Vieira, J. H., and J. K. Storrow, 1991, Z. Phys. C 51, 241.

Dasgupta, M., and B. R. Webber, 1997, Nucl. Phys. B 484, 247.

Dawson, S., R. K. Ellis, and P. Nason, 1988, Nucl. Phys. B 303, 607.

Dawson, S., R. K. Ellis, and P. Nason, 1989, Nucl. Phys. B 327, 49; 335, 260(E).

Decamp, D., et al. (ALEPH Collaboration), 1991, Phys. Lett. B 264, 476.

De Florian, D., and S. Frixione, 1999, Phys. Lett. B 457, 236.

De Florian, D., S. Frixione, A. Signer, and W. Vogelsang, 1999, Nucl. Phys. B 539, 455.

De Florian, D., and W. Vogelsang, 1998, Phys. Rev. D 57, 4376.

Derrick, M., et al. (ZEUS Collaboration), 1995a, Phys. Lett. B 342, 417.

Derrick, M., et al. (ZEUS Collaboration), 1995b, Phys. Lett. B 348, 665.

Derrick, M., et al. (ZEUS Collaboration), 1995c, Z. Phys. C 67, 227.

Derrick, M., et al. (ZEUS Collaboration), 1996, Phys. Lett. B 384, 401.

Derrick, M., et al. (ZEUS Collaboration), 1997a, in International Europhysics Conference on High Energy Physics: Proceedings, edited by D. Lellouch, G. Mikenberg, and E. Rabinovici (Springer-Verlag, Berlin), p. 535.

Derrick, M., et al. (ZEUS Collaboration), 1997b, in International Europhysics Conference on High Energy Physics: Proceedings, edited by D. Lellouch, G. Mikenberg, and E. Rabinovici (Springer-Verlag, Berlin), p. 541. 
De Witt, R. J., L. M. Jones, J. D. Sullivan, D. E. Willen, and H. W. Wyld, 1979, Phys. Rev. D 19, 2046; 20, 1751(E).

Drees, M., and R. M. Godbole, 1994, Phys. Rev. D 50, 3124.

Drees, M., and K. Grassie, 1985, Z. Phys. C 28, 451.

Drees, M., M. Krämer, P. M. Zerwas, and J. Zunft, 1993, Phys. Lett. B 306, 371.

Duke, D. W., and J. F. Owens, 1980, Phys. Rev. D 22, 2280.

Duke, D. W., and J. F. Owens, 1982, Phys. Rev. D 26, 1600.

Eboli, O. J., E. M. Gregores, and F. Halzen, 1999, Phys. Lett. B 451, 241.

Ellis, R. K., M. A. Furman, H. E. Haber, and I. Hinchliffe, 1980, Nucl. Phys. B 173, 397.

Ellis, R. K., H. Georgi, M. Machacek, H. D. Politzer, and G. G.

Ross, 1979, Nucl. Phys. B 152, 285.

Ellis, R. K., and P. Nason, 1989, Nucl. Phys. B 312, 551.

Ellis, R. K., D. A. Ross, and A. E. Terrano, 1981, Nucl. Phys. B 178, 421.

Ellis, R. K., and J. C. Sexton, 1986, Nucl. Phys. B 269, 445.

Ellis, S. D., Z. Kunszt, and D. E. Soper, 1989a, Phys. Rev. Lett. 62, 726.

Ellis, S. D., Z. Kunszt, and D. E. Soper, 1989b, Phys. Rev. D 40, 2188.

Ellis, S. D., Z. Kunszt, and D. E. Soper, 1990, Phys. Rev. Lett. 64, 2121.

Ellis, S. D., Z. Kunszt, and D. E. Soper, 1992, Phys. Rev. Lett. 69, 3615.

Ellis, S. D. and D. E. Soper, 1993, Phys. Rev. D 48, 3160.

Enomoto, R., et al. (TOPAZ Collaboration), 1994a, Phys. Rev. D 50, 1879.

Enomoto, R., et al. (TOPAZ Collaboration), 1994b, Phys. Lett. B 328, 535.

Erdmann, M., 1997, The Partonic Structure of the Photon, Springer Tracts in Modern Physics No. 138 (Springer, Berlin/ NY).

Ericson, T. E., B. Loiseau, and A. W. Thomas, 2000, CERNTH-2000-166. Also available as e-print hep-ph/0009312.

Fabricius, K., G. Kramer, G. Schierholz, and I. Schmitt, 1981, Z. Phys. C 11, 315.

Fermi, E., 1924, Z. Phys. 29, 315.

Field, J. H., F. Kapusta, and L. Poggioli, 1987, Z. Phys. C 36, 121.

Field, R. D., and R. P. Feynman, 1978, Nucl. Phys. B 136, 1.

Fleming, S., and T. Mehen, 1998, Phys. Rev. D 58, 037503.

Floratos, E. G., C. Kounnas, and R. Lacaze, 1981, Nucl. Phys. B 192, 417.

Fontannaz, M., J. P. Guillet, and G. Heinrich, 2001a, Eur. Phys. J. C 21, 303.

Fontannaz, M., J. P. Guillet, and G. Heinrich, 2001b, Eur. Phys. J. C 22, 303.

Fontannaz, M., J. P. Guillet, and G. Heinrich, 2002, Eur. Phys. J. C 23, 503.

Fontannaz, M., A. Mantrach, B. Pire, and D. Schiff, 1980, Z. Phys. C 6, 241.

Fontannaz, M., and E. Pilon, 1992, Phys. Rev. D 45, 382.

Forshaw, J. R., and R. G. Roberts, 1993, Phys. Lett. B 319, 539.

Frabetti, P. L., et al. (E687 Collaboration), 1993, Phys. Lett. B 308, 193.

Frabetti, P. L., et al. (E687 Collaboration), 1996, Phys. Lett. B 370, 222.

Frankfurt, L. L., and E. G. Gurvich, 1996, Phys. Lett. B 386, 379.

Fritzsch, H., 1977, Phys. Lett. 67B, 217.

Fritzsch, H., and K.-H. Streng, 1978, Phys. Lett. 72B, 385.
Frixione, S., 1998a, Phys. Lett. B 429, 369.

Frixione, S., 1998b, in ICHEP 1998: 29th International Conference on High-Energy Physics, Vancouver, Canada, edited by A. Astbury, D. Axen, and J. Robinson (World Scientific, Singapore), p. 790. Also available as hep-ph/9809397.

Frixione, S., 1999, Nucl. Phys. B, Proc. Suppl. 79, 608.

Frixione, S., M. Krämer, and E. Laenen, 2000a, Nucl. Phys. B 571, 169.

Frixione, S., M. Krämer, and E. Laenen, 2000b, J. Phys. G 26, 723.

Frixione, S., M. L. Mangano, P. Nason, and G. Ridolfi, 1993, Phys. Lett. B 319, 339.

Frixione, S., M. L. Mangano, P. Nason, and G. Ridolfi, 1994a, Nucl. Phys. B 412, 225.

Frixione, S., M. L. Mangano, P. Nason, and G. Ridolfi, 1994b, Nucl. Phys. B 431, 453.

Frixione, S., M. L. Mangano, P. Nason, and G. Ridolfi, 1995, Phys. Lett. B 348, 633.

Frixione, S., P. Nason, and G. Ridolfi, 1995, Nucl. Phys. B 454, 3.

Frixione, S., and G. Ridolfi, 1996, Phys. Lett. B 383, 227.

Frixione, S., and G. Ridolfi, 1997, Nucl. Phys. B 507, 315.

Furmanski, W., and R. Petronzio, 1980, Phys. Lett. 97B, 437.

Furmanski, W., and R. Petronzio, 1982, Z. Phys. C 11, 293.

Gastmans, R., and R. Meuldermans, 1973, Nucl. Phys. B 63, 277.

Gehrmann-De Ridder, A., T. Gehrmann, and E. W. Glover, 1997, Phys. Lett. B 414, 354.

Georgi, H., and H. D. Politzer, 1978, Nucl. Phys. B 136, 445. Giele, W. T., S. Keller, and E. Laenen, 1996, Phys. Lett. B 372, 141.

Giele, W. T., and W. B. Kilgore, 1997, Phys. Rev. D 55, 7183. Ginzburg, I. F., G. L. Kotkin, S. L. Panfil, V. G. Serbo, and V. I. Telnov, 1984, Nucl. Instrum. Methods Phys. Res. A 219, 5. Glück, M., J. F. Owens, and E. Reya, 1978, Phys. Rev. D 17, 2324.

Glück, M., and E. Reya, 1983, Phys. Rev. D 28, 2749.

Glück, M., and E. Reya, 1988, Z. Phys. C 39, 569.

Glück, M., E. Reya, and I. Schienbein, 1999a, Eur. Phys. J. C 10, 313 .

Glück, M., E. Reya, and I. Schienbein, 1999b, Phys. Rev. D 60, 054019; 62, 019902(E).

Glück, M., E. Reya, and I. Schienbein, 2001, Phys. Rev. D 63, 074008 .

Glück, M., E. Reya, and C. Sieg, 2001a, Phys. Lett. B 503, 285.

Glück, M., E. Reya, and C. Sieg, 2001b, Eur. Phys. J. C 20, 271.

Glück, M., E. Reya, and M. Stratmann, 1995, Phys. Rev. D 51, 3220.

Glück, M., E. Reya, and M. Stratmann, 1996, Phys. Rev. D 54, 5515.

Glück, M., E. Reya, and A. Vogt, 1992a, Phys. Rev. D 45, 3986. Glück, M., E. Reya, and A. Vogt, 1992b, Phys. Rev. D 46, 1973. Glück, M., E. Reya, and A. Vogt, 1993, Phys. Rev. D 48, 116; 51, 1427(E).

Glück, M., M. Stratmann, and W. Vogelsang, 1994, Phys. Lett. B 337, 373.

Glück, M., and W. Vogelsang, 1992, Z. Phys. C 55, 353.

Godbole, R. M., D. Indumathi, and M. Krämer, 2002, Phys. Rev. D 65, 074003.

Godbole, R. M., D. P. Roy, and K. Sridhar, 1996, Phys. Lett. B 373, 328.

Gordon, L. E., 1994, Phys. Rev. D 50, 6753.

Gordon, L. E., 1997a, Nucl. Phys. B 501, 175. 
Gordon, L. E., 1997b, Nucl. Phys. B 501, 197.

Gordon, L. E., 1997c, Phys. Lett. B 406, 184.

Gordon, L. E., 1998, Phys. Rev. D 57, 235.

Gordon, L. E., and J. K. Storrow, 1992a, Z. Phys. C 56, 307. Gordon, L. E., and J. K. Storrow, 1992b, Phys. Lett. B 291, 320. Gordon, L. E., and J. K. Storrow, 1994, Z. Phys. C 63, 581.

Gordon, L. E., and J. K. Storrow, 1996, Phys. Lett. B 385, 385. Gordon, L. E., and J. K. Storrow, 1997, Nucl. Phys. B 489, 405. Gordon, L. E., and W. Vogelsang, 1993, Phys. Rev. D 48, 3136. Gordon, L. E., and W. Vogelsang, 1994a, Phys. Rev. D 49, 170. Gordon, L. E., and W. Vogelsang, 1994b, Phys. Rev. D 50, 1901.

Gordon, L. E., and W. Vogelsang, 1995, Phys. Rev. D 52, 58. Gordon, L. E., and W. Vogelsang, 1996, Phys. Lett. B 387, 629. Greco, M., and S. Rolli, 1993, Z. Phys. C 60, 169.

Greco, M., and S. Rolli, 1995, Phys. Rev. D 52, 3853.

Greco, M., S. Rolli, and A. Vicini, 1995, Z. Phys. C 65, 277.

Greco, M., and A. Vicini, 1994, Nucl. Phys. B 415, 386.

Greub, C., H. Jung, D. Krucker, and D. Wyler, 1993, Z. Phys. C 60, 721 .

Groom, D. E., et al. (Particle Data Group Collaboration), 2000, Eur. Phys. J. C 15, 1.

Grunberg, G., 1980, Phys. Lett. 95B, 70; 110B, 501(E).

Gutbrod, F., G. Kramer, and G. Schierholz, 1984, Z. Phys. C 21, 235.

Hagiwara, K., T. Izubuchi, M. Tanaka, and I. Watanabe, 1995, Phys. Rev. D 51, 3197.

Halzen, F., 1977, Phys. Lett. 69B, 105.

Hanson, G., et al., 1975, Phys. Rev. Lett. 35, 1609.

Harris, B. W., M. Klasen, and J. Vossebeld, 1999, e-print hep-ph/9905348.

Harris, B. W., and J. F. Owens, 1997, Phys. Rev. D 56, 4007. Harris, B. W., and J. F. Owens, 1998, Phys. Rev. D 57, 5555. Harris, B. W., and J. F. Owens, 2001, Phys. Rev. D 65, 094032. Hassan, J. A., and D. J. Pilling, 1981, Nucl. Phys. B 187, 563. Hayashii, H., et al. (TOPAZ Collaboration), 1993, Phys. Lett. B 314, 149.

Heister, A., et al. (ALEPH Collaboration), 2001, Phys. Lett. B 512, 30.

Hill, C. T., and G. G. Ross, 1979, Nucl. Phys. B 148, 373.

Holtmann, H., J. Speth, and A. Szczurek, 1996, Nucl. Phys. A 569, 631.

Hoyer, P., N. Marchal, and S. Peigné, 2000, Phys. Rev. D 62, 114001.

Hoyer, P., and S. Peigné, 1999, Phys. Rev. D 59, 034011.

Huth, J. E., et al. in Research Directions for the Decade: Proceedings of the 1990 Summer Study on High Energy Physics, Snowmass, Colorado, edited by E. L. Berger (World Scientific, Singapore/River Edge, NJ), p. 134.

Irving, A. C., and D. B. Newland, 1980, Z. Phys. C 6, 27.

Jankowski, P., M. Krawczyk, and M. Wing, 2001, e-print hep-ph/0103330.

Japaridze, G., W. Nowak, and A. Tkabladze, 2000, Phys. Rev. D 62, 034022.

Japaridze, G., and A. Tkabladze, 1998, Phys. Lett. B 433, 139. Jikia, G., and A. Tkabladze, 1996, Phys. Rev. D 54, 2030.

Joos, H., 1967, Phys. Lett. 24B, 103.

Kamitani, T., and L. Rinolfi, 2001, CLIC-NOTE-465.

Karliner, M., and R. W. Robinett, 1994, Phys. Lett. B 324, 209.

Kartvelishvili, V. G., A. K. Likhoded, and V. A. Petrov, 1978,

Phys. Lett. 78B, 615.

Kessler, P., 1975, Acta Phys. Austriaca 41, 141.
Khalafi, F., P. V. Landshoff, and W. J. Stirling, 1983, Phys. Lett. 130B, 215.

Khan, H., and P. Hoodbhoy, 1996, Phys. Lett. B 382, 189.

Kim, B. J., et al. (AMY Collaboration), 1994, Phys. Lett. B 325, 248.

Kim, C. S., and E. Reya, 1993, Phys. Lett. B 300, 298.

Kinoshita, T., 1962, J. Math. Phys. 3, 650.

Klasen, M., 1996a, in Deep Inelastic Scattering and Related Phenomena (DIS-96): Proceedings, edited by G. D'Agostini and A. Nigro (World Scientific, Singapore), p. 286.

Klasen, M., 1996b, Ph.D. thesis (Hamburg University), DESY96-204.

Klasen, M., 1997a, in New Trends in HERA Physics: Proceedings 1997, Ringberg, Germany, edited by B. A. Kniehl, G. Kramer, and A. Wagner (World Scientific, Singapore), p. 236. Klasen, M., 1997b, in Workshop on Physics at the First Muon Collider and at the Front End of the Muon Collider, Batavia, IL, USA, edited by S. Geer and R. Raja, AIP Conf. Proc. No. 435 (AIP, Woodbury, NY), p. 495.

Klasen, M., 1999a, Eur. Phys. J. C 7, 225.

Klasen, M., 1999b, in New Trends in HERA Physics 1999, Ringberg, Germany, Lecture Notes in Physics No. 546, edited by G. Grindhammer, B. A. Kniehl, and G. Kramer (Springer, Berlin), p. 250.

Klasen, M., 2001a, in Deep Inelastic Scattering (DIS-2001), Bologna, Italy, e-print hep-ph/0106274.

Klasen, M., 2001b, in New Trends in HERA Physics 2001: Ringberg, Germany, edited by G. Grindhammer, B. A. Kniehl, G. Kramer, and W. Ochs (IOP, Bristol), p. 1091. Also published in J. Phys. G 28 (5), 727 (2002).

Klasen, M., 2001c, Eur. Phys. J. C 3, 1.

Klasen, M., T. Kleinwort, and G. Kramer, 1998, Eur. Phys. J. C $\mathbf{1}, 1$.

Klasen, M., B. A. Kniehl, L. Mihaila, and M. Steinhauser, 2001, Nucl. Phys. B 609, 518.

Klasen, M., B. A. Kniehl, L. Mihaila, and M. Steinhauser, 2002, Phys. Rev. Lett. 89, 032001.

Klasen, M., and G. Kramer, 1996a, Phys. Lett. B 366, 385.

Klasen, M., and G. Kramer, 1996b, Z. Phys. C 72, 107.

Klasen, M., and G. Kramer, 1996c, Phys. Lett. B 386, 384.

Klasen, M., and G. Kramer, 1997a, Z. Phys. C 76, 67.

Klasen, M., and G. Kramer, 1997b, Phys. Rev. D 56, 2702.

Klasen, M., and G. Kramer, 2001, Phys. Lett. B 508, 259.

Klasen, M., G. Kramer, and B. Pötter, 1998, Eur. Phys. J. C 1, 261.

Klasen, M., G. Kramer, and S. G. Salesch, 1995, Z. Phys. C 68, 113.

Kleinwort, T., and G. Kramer, 1996a, Phys. Lett. B 370, 141.

Kleinwort, T., and G. Kramer, 1996b, Nucl. Phys. B 477, 3.

Kleinwort, T., and G. Kramer, 1997, Z. Phys. C 75, 489.

Kniehl, B. A., 1997, in New Trends in HERA Physics 1997, Ringberg, Germany, edited by B. A. Kniehl, G. Kramer, and A. Wagner (World Scientific, Singapore), p. 260.

Kniehl, B. A., M. Krämer, G. Kramer, and M. Spira, 1995, Phys. Lett. B 356, 539.

Kniehl, B. A., and G. Kramer, 1994, Z. Phys. C 62, 53.

Kniehl, B. A., and G. Kramer, 1997a, Phys. Lett. B 413, 416.

Kniehl, B. A., and G. Kramer, 1997b, Phys. Rev. D 56, 5820.

Kniehl, B. A., and G. Kramer, 1999, Eur. Phys. J. C 6, 493.

Kniehl, B. A., G. Kramer, and B. Pötter, 2000a, Nucl. Phys. B 582, 514.

Kniehl, B. A., G. Kramer, and B. Pötter, 2000b, Phys. Rev. Lett. 85, 5288. 
Kniehl, B. A., G. Kramer, and B. Pötter, 2001, Nucl. Phys. B 597, 337.

Kniehl, B. A., G. Kramer, and M. Spira, 1997, Z. Phys. C 76, 689.

Ko, P., J. Lee, and H. S. Song, 1996, Phys. Rev. D 54, 4312; 60 , 119902(E).

Koller, K., T. F. Walsh, and P. M. Zerwas, 1979, Z. Phys. C 2, 197.

Krämer, M., 1996, Nucl. Phys. B 459, 3.

Krämer, M., 2001, Prog. Part. Nucl. Phys. 47, 141.

Krämer, M., and E. Laenen, 1996, Phys. Lett. B 371, 303.

Krämer, M., J. Steegborn, P. M. Zerwas, and J. Zunft, 1995,

Phys. Lett. B 348, 657.

Kramer, G., 1996, J. Phys. G 22, 717.

Kramer, G., 1998, in 1998 QCD and High Energy Hadronic Interactions, 33d rencontre de Moriond, edited by J. Tran Thanh Van (Gioi, Vietnam).

Kramer, G., 1999, in New Trends in HERA Physics 1999, Ringberg, Germany, Lecture Notes in Physics No. 546, edited by G. Grindhammer, B. A. Kniehl, and G. Kramer (Springer, Berlin), p. 275.

Kramer, G., and B. Lampe, 1989, Fortschr. Phys. 37, 161.

Kramer, G., and B. Pötter, 1998, Eur. Phys. J. C 5, 665.

Kramer, G., and B. Pötter, 1999, Phys. Lett. B 453, 295.

Kramer, G., and S. G. Salesch, 1993, Phys. Lett. B 317, 218.

Kramer, G., and S. G. Salesch, 1994a, Z. Phys. C 61, 277.

Kramer, G., and S. G. Salesch, 1994b, Phys. Lett. B 333, 519.

Kramer, G., and H. Spiesberger, 2001, Eur. Phys. J. C 22, 289.

Krawczyk, M., M. Staszel, and A. Zembrzuski, 2001, Phys.

Rep. 345, 265.

Krawczyk, M., and A. Zembrzuski, 1998, Phys. Rev. D 57, 10. Krawczyk, M., and A. Zembrzuski, 2001a, Phys. Rev. D 64, 114017.

Krawczyk, M., and A. Zembrzuski, 2001b, IFT-2001-10. Also available as e-print hep-ph/0103344.

Kretzer, S., 2000, Phys. Rev. D 62, 054001.

Krüger, K., 2000, in 7th International Workshop on Deep Inelastic Scattering (DIS 2000), Liverpool, England, edited by J.

A. Gracey and T. Greenshow (World Scientific, Singapore), p. 368.

Kühn, J. H., E. Mirkes, and J. Steegborn, 1993, Z. Phys. C 57, 615.

Kunszt, Z., and D. E. Soper, 1992, Phys. Rev. D 46, 192.

Laenen, E. S., S. Riemersma, J. Smith, and W. L. van Neerven, 1994, Phys. Rev. D 49, 5753.

Laenen, E., G. Sterman, and W. Vogelsang, 2000, Phys. Rev. Lett. 84, 4296.

Lai, H. L., et al. (CTEQ Collaboration), 1995, Phys. Rev. D 51, 4763.

Lai, H. L., et al. (CTEQ Collaboration), 2000, Eur. Phys. J. C 12, 375 .

Lee, S. W., et al. (ZEUS collaboration), 2000, Nucl. Phys. B, Proc. Suppl. 82, 162.

Lee, T. D., and M. Nauenberg, 1964, Phys. Rev. 133, B1549.

Leibbrandt, G., 1975, Rev. Mod. Phys. 47, 849.

Lepage, G. P., L. Magnea, U. Magnea, C. Nakhleh, and K. Hornbostel, 1992, Phys. Rev. D 46, 4052.

Linsel, F., 1995, Ph.D. thesis (Hamburg University), DESYFH1T-95-03.

Llewellyn Smith, C. H., 1978, Phys. Lett. 79B, 83.

Ma, J. P., 1994, Phys. Lett. B 332, 398.

Ma, J. P., 1995, Nucl. Phys. B 447, 405.

Ma, J. P., 1996a, Phys. Rev. D 53, 1185.
Ma, J. P., 1996b, Nucl. Phys. B 460, 109.

Ma, J. P., 1997, Nucl. Phys. B 498, 267.

Ma, J. P., B. H. McKellar, and C. B. Paranavitane, 1998, Phys. Rev. D 57, 606.

Ma, J. P., B. H. McKellar, and C. B. Paranavitane, 2000, Phys. Rev. D 61, 114502.

Maltoni, F., M. L. Mangano, and A. Petrelli, 1998, Nucl. Phys. B 519, 361.

Mangano, M. L., P. Nason, and G. Ridolfi, 1992, Nucl. Phys. B 373, 295.

Marciano, W. J., 1975, Phys. Rev. D 12, 3861.

Marciano, W. J., and A. Sirlin, 1975, Nucl. Phys. B 88, 86.

Martin, A. D., R. G. Roberts, and W. J. Stirling, 1993, Phys. Lett. B 306, 145; 309, 492(E).

Martin, A. D., R. G. Roberts, W. J. Stirling, and P. J. Sutton, 1992, Phys. Rev. D 45, 2349.

Martin, A. D., R. G. Roberts, W. J. Stirling, and R. S. Thorne, 2000, Eur. Phys. J. C 14, 133.

Martin, A. D., R. G. Roberts, W. J. Stirling, and R. S. Thorne, 2001, in 8th International Workshop on Deep Inelastic Scattering (DIS-2001), Bologna, Italy (in press).

Mehen, T., 1997, Phys. Rev. D 55, 4338.

Mele, B., and P. Nason, 1991, Nucl. Phys. B 361, 626.

Mertig, R., and W. L. van Neerven, 1996, Z. Phys. C 70, 637.

Morii, T., D. Roy, and K. Sudoh, 2000, Phys. Rev. D 62, 057501.

Morii, T., and K. Sudoh, 2000, Phys. Rev. D 62, 057501.

Moroni, L., et al. (E687 Collaboration), 1994, Nuovo Cimento A 107, 2025.

Narison, S., G. M. Shore, and G. Veneziano, 1993, Nucl. Phys. B 391, 69.

Nason, P., and C. Oleari, 1999, Phys. Lett. B 447, 327.

Nason, P., and C. Oleari, 2000, Nucl. Phys. B 565, 245.

Nason, P., and B. R. Webber, 1994, Nucl. Phys. B 421, 473; 480, 755(E).

Nisius, R., 2000, Phys. Rep. 332, 165.

Norrbin, E., and T. Sjöstrand, 2000, Eur. Phys. J. C 17, 137.

Ohyama, H., et al. (VENUS Collaboration), 1997, Z. Phys. C 75, 209.

Owens, J. F., 1980, Phys. Rev. D 21, 54.

Owens, J. F., 1987, Rev. Mod. Phys. 59, 465.

Passarino, G., and M. Veltman, 1979, Nucl. Phys. B 160, 151.

Paul, E., 1992, in Proceedings of the 21st International Symposium on Multiparticle Dynamics, Wuhan, China, edited by Y. $\mathrm{Wu}$ and L. Liu (World Scientific, Singapore/River Edge, N.J.). Also available as DESY-92-026.

Peterson, C., D. Schlatter, I. Schmitt, and P. Zerwas, 1983, Phys. Rev. D 27, 105.

Philipsen, O., 1992, Z. Phys. C 54, 643.

Pötter, B., 1999a, Nucl. Phys. B 540, 382.

Pötter, B., 1999b, Nucl. Phys. B 559, 323.

Pötter, B., 1999c, Eur. Phys. J. C 5, 1.

Qiu, J., and X. Zhang, 2001, Phys. Rev. D 64, 074007.

Quigg, C., and J. L. Rosner, 1979, Phys. Rep. 56, 167.

Ratcliffe, P., 1983, Nucl. Phys. B 223, 45.

Rijken, P. J., and W. L. van Neerven, 1996, Phys. Lett. B 386, 422.

Rijken, P. J., and W. L. van Neerven, 1997, Nucl. Phys. B 487, 233.

Ross, M., and L. Stodolsky, 1966, Phys. Rev. 149, 1172.

Rossi, G., 1983, Phys. Lett. 130B, 105.

Rossi, G., 1984, Phys. Rev. D 29, 852. 
Roudeau, P., et al. (WA4 Collaboration), 1980, in Elementary Constituents and Hadronic Structure, edited by J. Tran Thanh Van (Editions Frontières, Dreux, France), p. 277.

Sakurai, J. J., and D. Schildknecht, 1972, Phys. Lett. 40B, 121. Sasaki, K., and T. Uematsu, 1999, Phys. Rev. D 59, 114011.

Sasaki, K., and T. Uematsu, 2000, Phys. Lett. B 473, 309.

Sasaki, K., and T. Uematsu, 2001, Eur. Phys. J. C 20, 283.

Schroeder, D. V., 1990, Ph.D. thesis (Stanford University), SLAC-0371.

Schuler, G. A., and T. Sjöstrand, 1995, Z. Phys. C 68, 607.

Schuler, G. A., and T. Sjöstrand, 1996, Phys. Lett. B 376, 193.

Seymour, M. H., 1998, Nucl. Phys. B 513, 269.

Sjöstrand, T., 1994, Comput. Phys. Commun. 82, 74.

Smith, J., and W. L. van Neerven, 1992, Nucl. Phys. B 374, 36. Sterman, G., and S. Weinberg, 1977, Phys. Rev. Lett. 39, 1436. Stevenson, P. M., 1981a, Phys. Lett. 100B, 61.

Stevenson, P. M., 1981b, Phys. Rev. D 23, 2916.

Stodolsky, L., 1964, Phys. Rev. 134, B1099.

Stodolsky, L., 1967, Phys. Rev. Lett. 18, 135.

Stratmann, M., and W. Vogelsang, 1996, Phys. Lett. B 386, 370. Stratmann, M., and W. Vogelsang, 1997, Z. Phys. C 74, 641.

Suzuki, M., 1986, Phys. Rev. D 33, 676.

Takashimizu, N., et al. (AMY Collaboration), 1996, Phys. Lett. B 381, 372.
Telnov, V. I., 1990, Nucl. Instrum. Methods Phys. Res. A 294, 72.

't Hooft, G., and M. Veltman, 1972, Nucl. Phys. B 44, 189.

't Hooft, G., and M. Veltman, 1973, in Particle Interactions at Very High Energies, Part B, edited by D. Speiser, F. Halzen, and J. Weyers (Plenum, N.Y.), p. 177.

't Hooft, G., and M. Veltman, 1979, Nucl. Phys. B 153, 365.

Todorova-Nova, S., 2001, in Proceedings of the 31st International Symposium on Multiparticle Dynamics, Datong, China, e-print hep-ph/0112050.

Uehara, S., et al. (VENUS Collaboration), 1994, Z. Phys. C 63, 213.

Uematsu, T., and T. F. Walsh, 1981, Phys. Lett. 101B, 263.

Uematsu, T., and T. F. Walsh, 1982, Nucl. Phys. B 199, 93.

Vogelsang, W., 1996a, Phys. Rev. D 54, 2023.

Vogelsang, W., 1996b, Nucl. Phys. B 475, 47.

Vogelsang, W., and A. Vogt, 1995, Nucl. Phys. B 453, 334.

von Weizsäcker, C. F., 1934, Z. Phys. 88, 612.

Wengler, 2002, QCD and Hadronic Interactions, edited by J. Tran Thanh Van (Edition Frontières, Dreux, France) (in press); e-print hep-ex/0205074.

Williams, E. J., 1934, Phys. Rev. 45, 729.

Wing, M., 2001, e-print hep-ph/0103329.

Witten, E., 1977, Nucl. Phys. B 120, 189.

Xu, Z., 1984, Phys. Rev. D 30, 1440. 